University of Louisville ThinkIR: The University of Louisville's Institutional Repository

Electronic Theses and Dissertations

$5-2019$

\title{
Synthesis, characterization, and evaluation of metal complexes with cancer selective anti-proliferative effects and hydrogen evolution catalytic properties.
}

Nicholas Vishnosky

University of Louisville

Follow this and additional works at: https://ir.library.louisville.edu/etd

Part of the Catalysis and Reaction Engineering Commons, and the Inorganic Chemicals Commons

\section{Recommended Citation}

Vishnosky, Nicholas, "Synthesis, characterization, and evaluation of metal complexes with cancer selective anti-proliferative effects and hydrogen evolution catalytic properties." (2019). Electronic Theses and Dissertations. Paper 3220.

https://doi.org/10.18297/etd/3220 accepted for inclusion in Electronic Theses and Dissertations by an authorized administrator of ThinkIR: The University of Louisville's Institutional Repository. This title appears here courtesy of the author, who has retained all other copyrights. For more information, please contact thinkir@louisville.edu. 
SYNTHESIS, CHARACTERIZATION, AND EVALUATION OF METAL COMPLEXES WITH CANCER SELECTIVE ANTI-PROLIFERATIVE EFFECTS AND HYDROGEN EVOLUTION CATLAYITC PROPERTIES

By

Nicholas Vishnosky

M.S., University of Louisville 2017

\author{
A Dissertation \\ Submitted to the Faculty of the \\ College of Arts and Sciences of the University of Louisville \\ in Partial Fulfillment of the Requirements \\ for the Degree of \\ Doctor of Philosophy \\ in Chemistry
}

Department of Chemistry

University of Louisville

Louisville, Kentucky

May 2019 
(C) 2018 by Nicholas Vishnosky

All rights reserved 

SYNTHESIS, CHARACTERIZATION, AND EVALUATION OF METAL COMPLEXES WITH CANCER SELECTIVE ANTI-PROLIFERATIVE EFFECTS AND HYDROGEN EVOLUTION CATLAYITC PROPERTIES

$$
\text { By }
$$

Nicholas Vishnosky

M.S., University of Louisville, 2017

\section{A Dissertation Approved on}

November 19, 2018

By the following Dissertation Committee

Dr. Craig A. Grapperhaus

(Dissertation Director)

Dr. Robert M. Buchanan

Dr. Eugene Mueller

Dr. Paula J. Bates 


\section{DEDICATION}

This dissertation is dedicated to my parents Catherine and William Vishnosky and my late grandparents, Andrew and Helene Siena, and Frances and William Vishnosky 


\section{ACKNOWLEDGEMENTS}

I would like to acknowledge Dr. Grapperhaus and Dr. Buchanan for their support and guidance during my time as a graduate student. I have learned valuable lessons from them in chemistry as well as a person, I will always be grateful. I would also like to thank my labmates, Oleksandr, Caleb, Kritika, Steve, Parabathi, Nina, and Peter for the discussions and camaraderie in the lab. I am thankful for the mentoring and examples shown by my previous labmates Dr. Chauhan, Dr. Kumar, Dr. Haddad, Dr. Zhang and Dr. Jain. Further I am thankful for the assistance of Dr. Stolowich with performing NMR experiments and Dr. Mashuta for his expertise in crystallography and taking the time to teach me. I would also like to thank my girlfriend Arizza Ibanez for her love and support as during our mutual studies. Lastly, I would like to thank my parents, for their unwavering support in my pursuit of chemistry throughout my life, I am eternally grateful and indebted to them. 


\begin{abstract}
SYNTHESIS, CHARACTERIZATION, AND EVALUATION OF METAL COMPLEXES FOR SELECTIVE TARGETING AND ANTI-PROLIFERATIVE EFFECT ON CANCER CELLS AND THEIR HYDROGEN EVOLUTION CATALYTIC PROPERTIES

Nicholas Vishnosky
\end{abstract}

November 19, 2018

Bis-thiosemicarbazones (BTSC) and their metal chelates have properties that are useful in several different scientific fields. These systems have already received attention in major fields of biology and engineering. Hydrogen evolution reaction (HER) catalysts need to be cheap and operate under minimal overpotentials with a long lifetime. The treatment of cancer requires, novel agents that have potent cytotoxic activity against cancer cells while displaying minimal side effects. In this dissertation the modular synthesis of these bis-thiosemicarbazone systems is utilized to regulate the redox chemistry for employment in the desired sector of chemistry. The ligand and metal chelates synthesized were characterized via cyclic voltammetry, NMR, UV-visible spectroscopy, FT-IR, EPR, and single crystal X-ray crystallography. The first generation replaced the pendent amine functionality of BTSCs with an alkoxy group. This replacement allowed for the structure to retain its physical characteristics that make these systems of interest in biological settings. However the change in functional group allows for tuning of the reduction potential, which is crucial for their activity. The copper 
diacetyl-bis(4-methylthiosemicarbazonato) (CuATSM) analog lays $250 \mathrm{mV}$ more anodic for its $\mathrm{Cu}^{\mathrm{II} / \mathrm{I}}$ couple.

Immobilization of NiATSM (2), NiATSDM (28), and NiATSM-F 6 (29) on a glassy carbon electrode surface revealed that after reductive cycling, 200-300 cycles, improved the overpotentials of $\mathbf{2}$ and $\mathbf{2 8}$ by $250 \mathrm{mV}$ from $700 \mathrm{mV}$ to $450 \mathrm{mV}$. The lack of a dramatic effect for $\mathbf{2 9}$, is due to solid state interactions between molecules and electrode surface. Raman spectroscopy and SEM reveal that 29, does not remain on the electrode surface, whereas $\mathbf{2}$ and $\mathbf{2 8}$ undergo dynamic rearrangement to improve overall performance.

A combination of both the pendant amine and alkoxy functionalities gave rise to the a new BTSC analog which chelated $\mathrm{Ni}, \mathrm{Cu}$ and $\mathrm{Zn}$. The electrochemical characterization revealed the copper chelates to have reduction potentials around $0.950 \mathrm{~V}$ $\mathrm{Cu}^{\mathrm{II} / \mathrm{I}}$. 3-(4,5-dimethylthiazol-2-yl)-2,5-diphenyltetrazolium bromide (MTT) assays showed high selectivity and potency with $50 \%$ growth inhibitory (GI50) concentrations of 0.09 and $2.0 \mu \mathrm{M}$ for carcinogenic and healthy cell lines respectively. Further the National Cancer Institute (NCI) 60 profile demonstrates that the copper chelate is effective against a wide variety of cancer types. 


\section{TABLE OF CONTENTS}

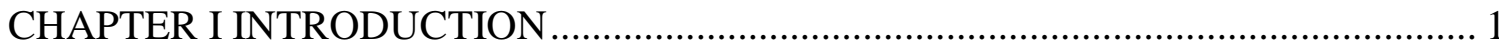

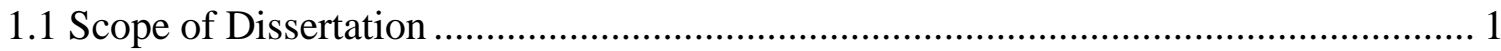

1.2 Synthesis of BSTCs and Radioimaging Applications ................................................. 4

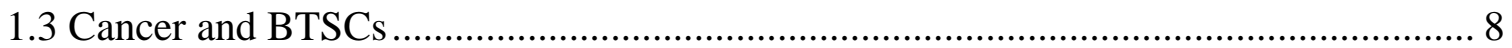

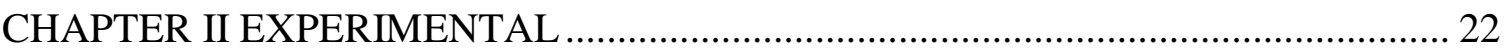

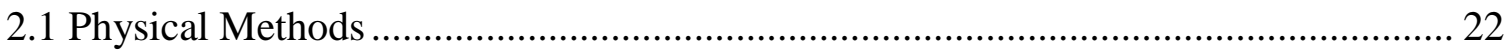

2.2 Electrochemical Measurements ....................................................................... 22

2.3 Synthetic Procedures............................................................................................ 24

2.5 Preparation of Modified Glassy Carbon Electrodes ................................................... 40

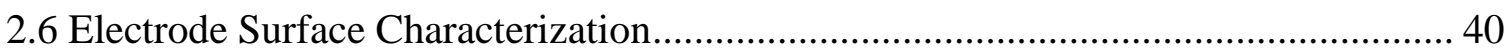

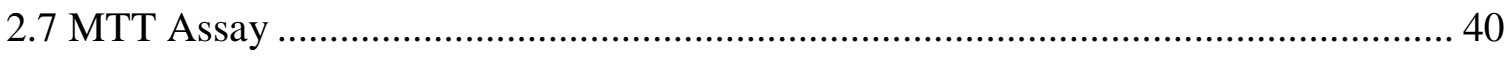

CHAPTER III SYNTHESES, STRUCTURES, AND ELECTROCHEMICAL STUDIES OF $N, N$-BIS(ALKYLTHIOCARBAMATE)BUTANE-2,3-DIIMINE CU(II) COMPLEXES AS PENDANT ALKOXY DERIVATIVES OF CU(ATSM) ................. 42

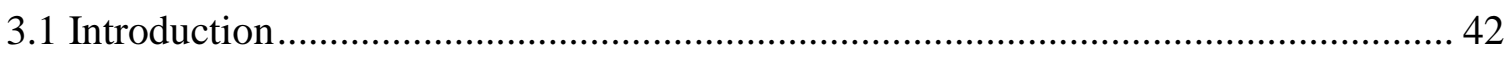

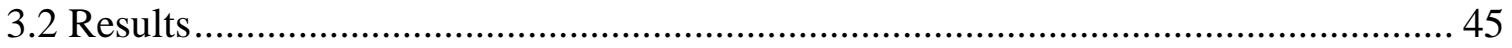

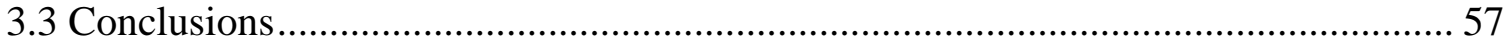

CHAPTER IV EFFECT OF STACKING INTERACTION ON THE TRANSLATION OF STRUCTURALLY RELATED BIS-THIOSEMICARBAZONE NI(II) HER CATALYSTS TO MODIFIED ELECTRODE SURFACES ........................................... 58

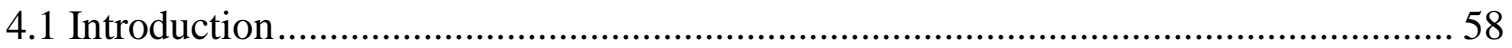

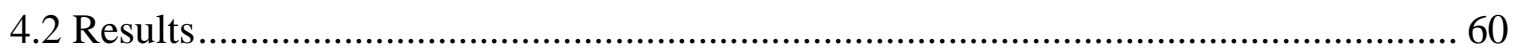

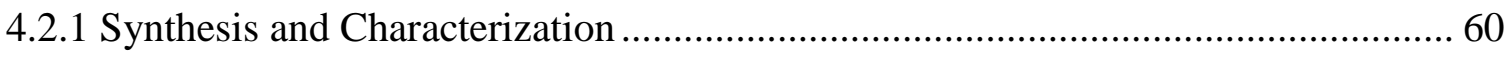

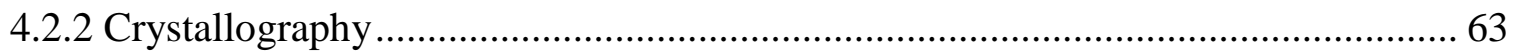

4.2.3 Homogeneous Electrochemical Characterization ................................................... 68

4.2.4 HER Activity of Modified Electrodes .................................................................. 69

4.2.5 Surface Characterization of Modified Electrodes...................................................... 74 
4.3 Conclusions

CHAPTER V SYNTHESIS, CHARACTERIZATION, AND BIOLOGICAL ACTIVITY OF NOVEL ASYMMETRIC BIS-THIOSEMICARBAZONE

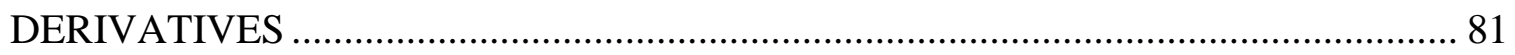

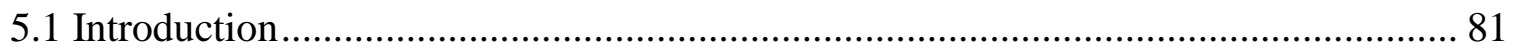

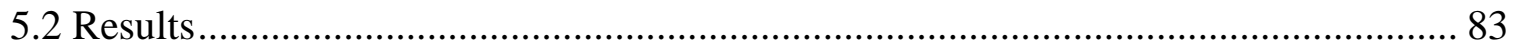

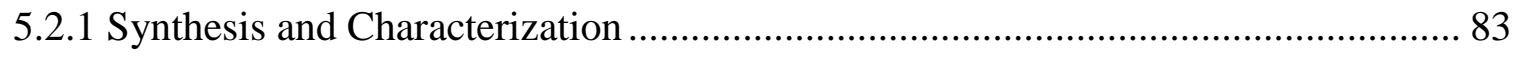

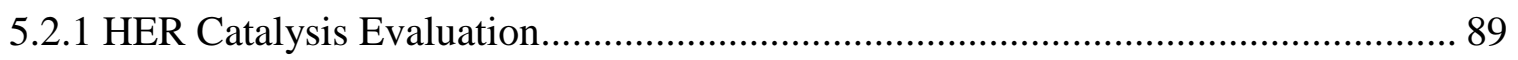

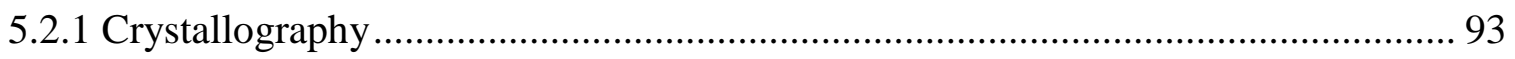

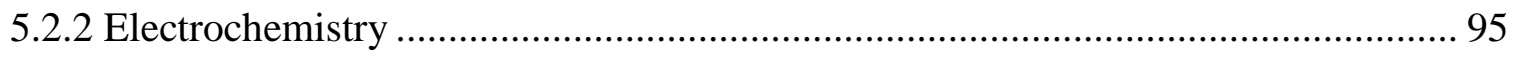

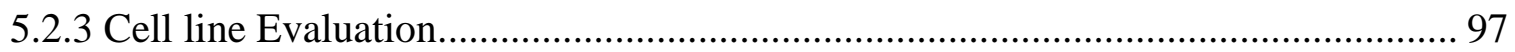

5.2.4 National Cancer Institute 60 Cell Line ............................................................... 102

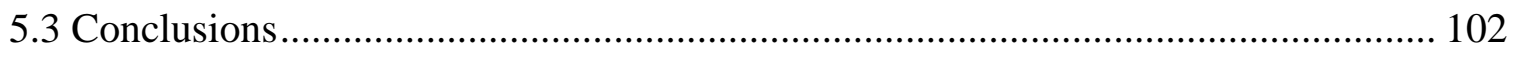

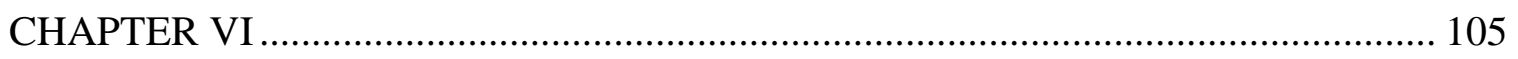

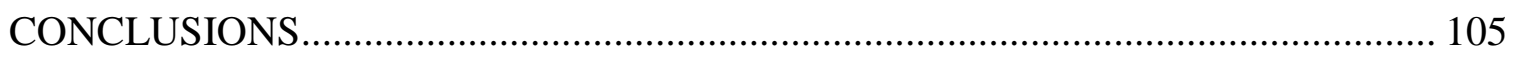

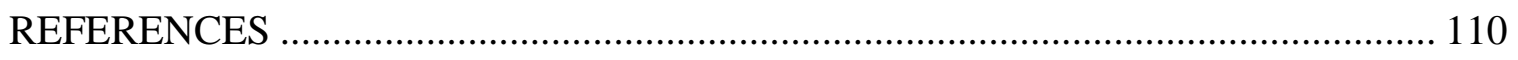

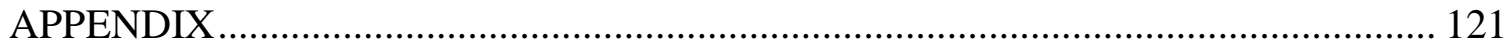

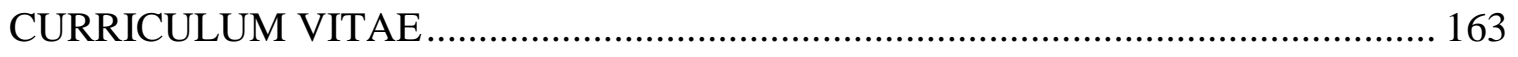




\section{LIST OF FIGURES}

\section{FIGURE}

Figure 1-1. Numbering scheme for all complexes.....................................................2

Figure 1-2. Modular synthesis of symmetric condensation (A) and asymmetric

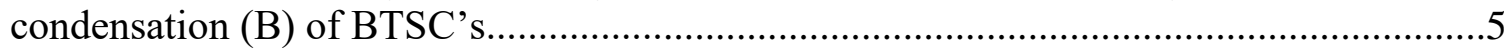

Figure 1-3. . Structure of CuATSM and CuGTSM .....................................................6

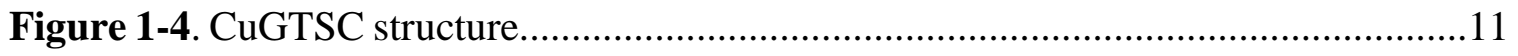

Figure 1-5. Distribution of energy consumption in the US........................................13

Figure 1-6. Structure of NiATSM para anisole..................................................... 17

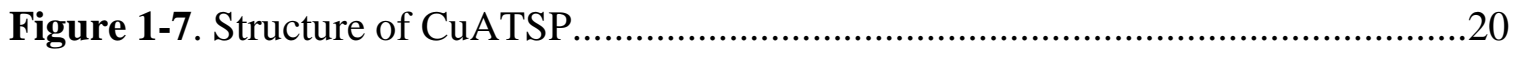

Figure 3-1. Depiction of copper retention in normoxic (A) and hypoxic (B) cells............43

Figure 3-2. Structural comparison of CuATSM, CuPTSM, and CuGTSM......................43

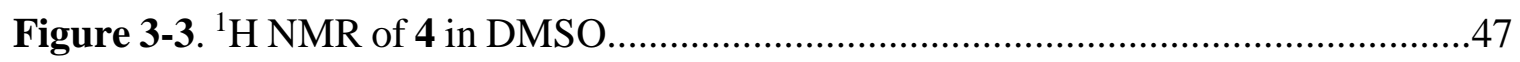

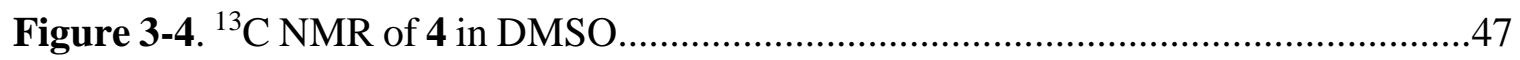

Figure 3-5. FT-IR spectra of $\mathbf{4}(\mathrm{A})$ and its copper complex 10 (B) ..............................48

Figure 3-6. UV-visible spectrum of 10, representative of the class................................49

Figure 3-7. Experimental (solid black line) and simulated (dashed red line) EPR spectrum of 12. This spectrum is representative of the whole class of compounds............................50

Figure 3-8. ORTEP view (50\% probability) of $\mathbf{1 0}$ showing atom labeling for all nonhydrogen atoms in the asymmetric unit and symmetry generated $(1-\mathrm{x},-\mathrm{y}, 1 / 2-\mathrm{z})$ donor atoms.

Figure 3-9. Representation of the conjugated $\pi$-network of the $N, N^{\prime}$ bis(alkylthiocarbamate)butane-2,3-diimine ligand of $\mathbf{1 0}$ highlighting localization of the $\mathrm{C}=\mathrm{N}$ bonds .54

Figure 3-10 ORTEP view (50\% probability) of $\mathbf{1 4}$ showing atom labeling for all nonhydrogen atoms in the asymmetric unit and symmetry generated $(1-\mathrm{x},-\mathrm{y}, 1 / 2-\mathrm{z})$ donor atoms. . .55 
Figure 3-11. Cyclic voltammogram of 10 in $\mathrm{CH}_{2} \mathrm{Cl}_{2}$ with $0.1 \mathrm{M}$ tetrabutylammonium hexafluorophosphate as supporting electrolyte. Scan rate $=100 \mathrm{mV} / \mathrm{s}$; potentials referenced versus $\mathrm{Fc}^{+} / \mathrm{Fc}$. .56

Figure 4-1. ${ }^{1} \mathrm{H}$ NMR of $\mathbf{2 8}$ (top) and 29 (bottom) in DMSO........................................61

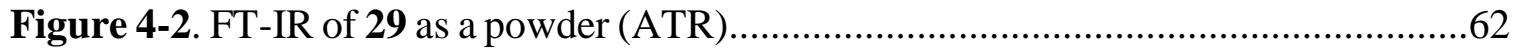

Figure 4-3. UV-visible spectra of 2 (black dots), 28 (solid blue), and 29 (dashed red) in acetonitrile. .63

Figure 4-4. ORTEP view (50\% probability) of 28 (top) and 29 (bottom) showing atom labeling for all non-hydrogen atoms in the asymmetric unit .64

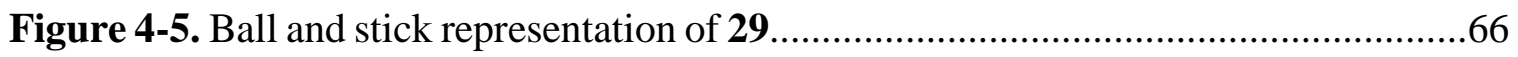

Figure 4-6. Packing diagrams for 2 (top), 28 (middle), and 29 (bottom).......................67

Figure 4-7. Cyclic voltammograms of $\mathbf{2}$ (black trace), $\mathbf{2 8}$ (blue trace), and $\mathbf{2 9}$ (red trace)..68

Figure 4-8. Polarization and Tafel plots of GC-1 to GC-3 during reductive cycling........70

Figure 4-9. Extended cycling effects on polarization curves and tafel plots for GC-1 to GC-3. .72

Figure 4-10. Comparison of GC-1 to GC-3 at peak activity .73

Figure 4-11. Cyclic voltammograms of 2, 28, and 29 under homogeneous HER catalytic conditions. .73

Figure 4-12. SEM images of GC-1 to GC-3 before and after cycling to peak activity........75

Figure 4-13. SEM image of GC-2 after 200 cycles, increased resolution.........................75

Figure 4-14. Raman spectra of 2, 28, and 29 as powders, and GC-1 to GC-3 before and after cycling. .77

Figure 4-15. Comparison of GC-3 before and after reductive cycling to glassy carbon.....78

Figure 4-16. Equivalent circuit model used to fit EIS data. .78

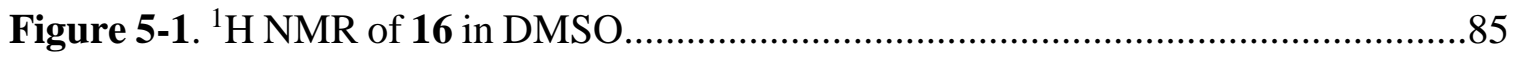

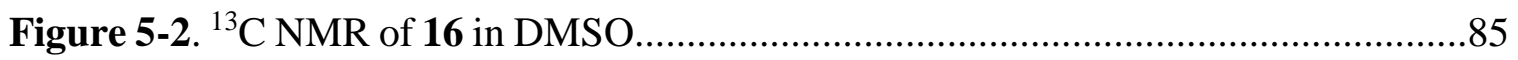

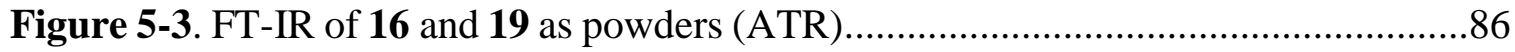

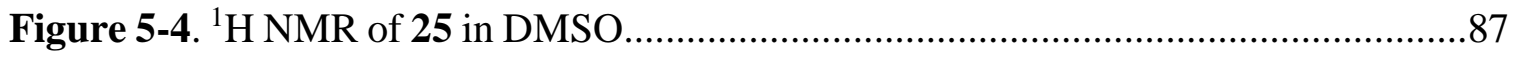

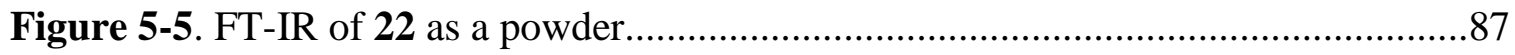

Figure 5-6. UV-visible spectra of 19 (blue trace), 20 (red trace), and 21 (black trace) recorded in acetonitrile.

Figure 5-7. Comparison of 21 and 10 under catalytic HER conditions .90 
Figure 5-8. Selected UV-Vis spectra of 1 during titration (top) Corrected absorbance vs additions of acid for 480 and $460 \mathrm{~nm}$.

Figure 5-9. Selected UV-Vis spectra of 21 during titration (top) Corrected absorbance vs additions of acid for 490 and $450 \mathrm{~nm}$. .92

Figure 5-10. ORTEP representations of 19 (A), 22 (B), 21 (C), and 18 (D)..... .94

Figure 5-11. Cyclic voltammograms over lays of ligand framework 16 (red trace) and 18 (blue trace) with their respective chelates copper (A), nickel (B), zinc (C) and, free ligand (D). .96

Figure 5-12. MTT assay results of cell lines A549 (top) and IMR90 (bottom) for the free ligand 16 (black trace), Cu complex 19 (red trace), Ni complex 22 (green trace) and Zn complex 25 (blue trace). 100

Figure 5-13. MTT assay results of cell lines of the ligand systems and their respective chelates 17 (A) and 18 (B) A549 (top) and IMR90 (bottom). .101

Figure 5-14. NCI 60 profile of 19 .103

Figure 5-15. NCI 60 profile of 21 .104 


\section{LIST OF SCHEMES}

\section{SCHEME}

Scheme 1-1. ZnATSM possible HER mechanisms........................................................15

Scheme 1-2. Mechanism for $\mathrm{H}_{2}$ evolution from CuATSM.............................................16

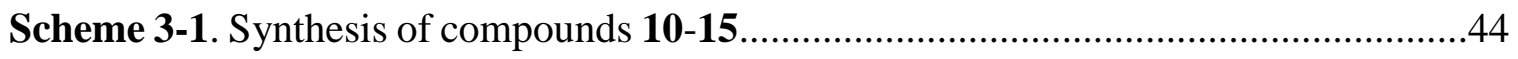

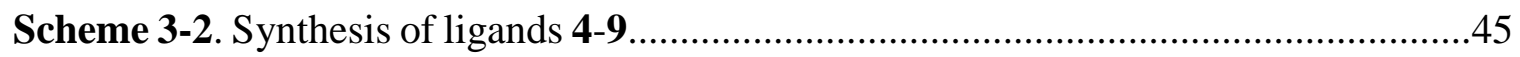

Scheme 4-1. Representation of Tafel slope steps.................................................. 71

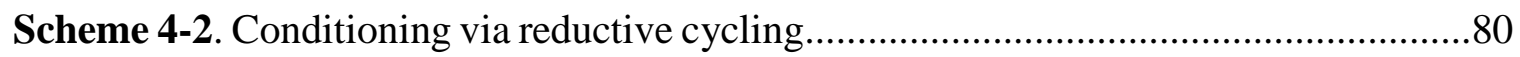

Scheme 5-1. Synthesis and numbering for ligands and metal chelates ..........................83

Scheme 5-2. Proposed mechanism of action for anti-cancer effects from copper

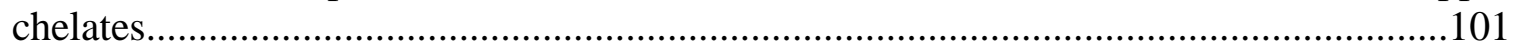




\section{LIST OF TABLES}

\section{TABLE}

Table 2-1. CCDC and publication reference information..........................................35

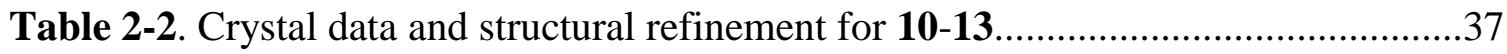

Table 2-3. Crystal data and structural refinement for 14, 15, 18, and 19......................38

Table 2-4. Crystal data and structural refinement for 21, 22, 28, and 29.....................39

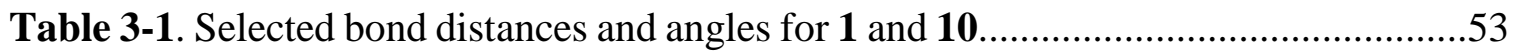

Table 3-2. UV-visible, EPR, and electrochemical data for 7-12 and CuATSM

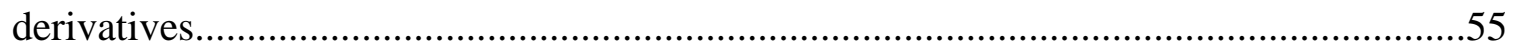

Table 4-1. Selected bond distances and angles for 2, 28, and 29...............................65

Table 4-2. Reduction potentials $\mathrm{vs} \mathrm{Fc}^{+} / \mathrm{Fc}$ for 2, 28, and 29.....................................69

Table 5-1. Selected bond distances and angles for 18, 19, 21, and 22.........................95

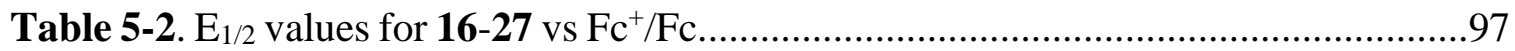

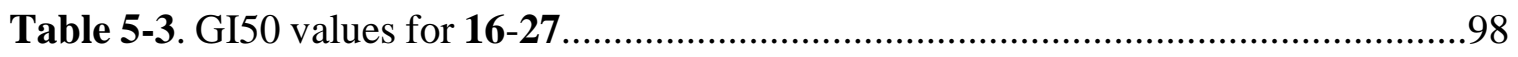




\section{CHAPTER I}

\section{INTRODUCTION}

\subsection{Scope of Dissertation}

This dissertation describes a series of three studies on the synthesis, characterization, and various applications of $\mathrm{N}_{2} \mathrm{~S}_{2}$ chelates related to the well-known bisthiosemicarbazone (BTSC) diacetyl-bis(4-methylthiosemicarbazone), $\mathrm{H}_{2}$ ATSM. These studies include: 1) the synthesis and characterization of a new class of $\mathrm{N}_{2} \mathrm{~S}_{2}$ chelates and their $\mathrm{Cu}(\mathrm{II})$ complexes; 2) the synthesis and application of nickel(II) BTSCs as heterogeneous catalysts for the hydrogen evolution reaction (HER); 3) the synthesis and evaluation of hybrid $\mathrm{N}_{2} \mathrm{~S}_{2}$ chelates and their metal complexes as anti-cancer therapeutics. A comprehensive list of the $29 \mathrm{~N}_{2} \mathrm{~S}_{2}$ compounds synthesized and evaluated in this dissertation can be found in Figure 1-1.

New ligands and metal complexes developed in these studies were characterized by nuclear magnetic resonance $\left({ }^{1} \mathrm{H}\right.$ NMR and ${ }^{13} \mathrm{C}$ NMR), Fourier-transform infrared (FTIR), and UV-Vis spectroscopies, mass spectrometry (ESI-MS), elemental analysis, single crystal x-ray crystallography, and cyclic voltammetry. Additionally, copper (II) complexes were characterized by electron paramagnetic resonance (EPR) spectroscopy. For compounds evaluated as heterogeneous HER catalysts additional characterization techniques were employed. These include linear sweep voltammetry (LSV) for complexes deposited on the electrode surface, Raman spectroscopy, scanning electron microscopy (SEM), and electrical impedance frequency response analysis (FRA). Compounds 
investigated as anti-cancer therapeutics were subjugated to dose response analysis from 3(4,5-dimethylthiazol-2-yl)-2,5-diphenyltetrazolium bromide (MTT) assays. Compounds 19 and 21 were screened by the National Cancer Institute (NCI) against a set of 60 cell lines to examine potency against a standardized set of a cell lines and cancer types.
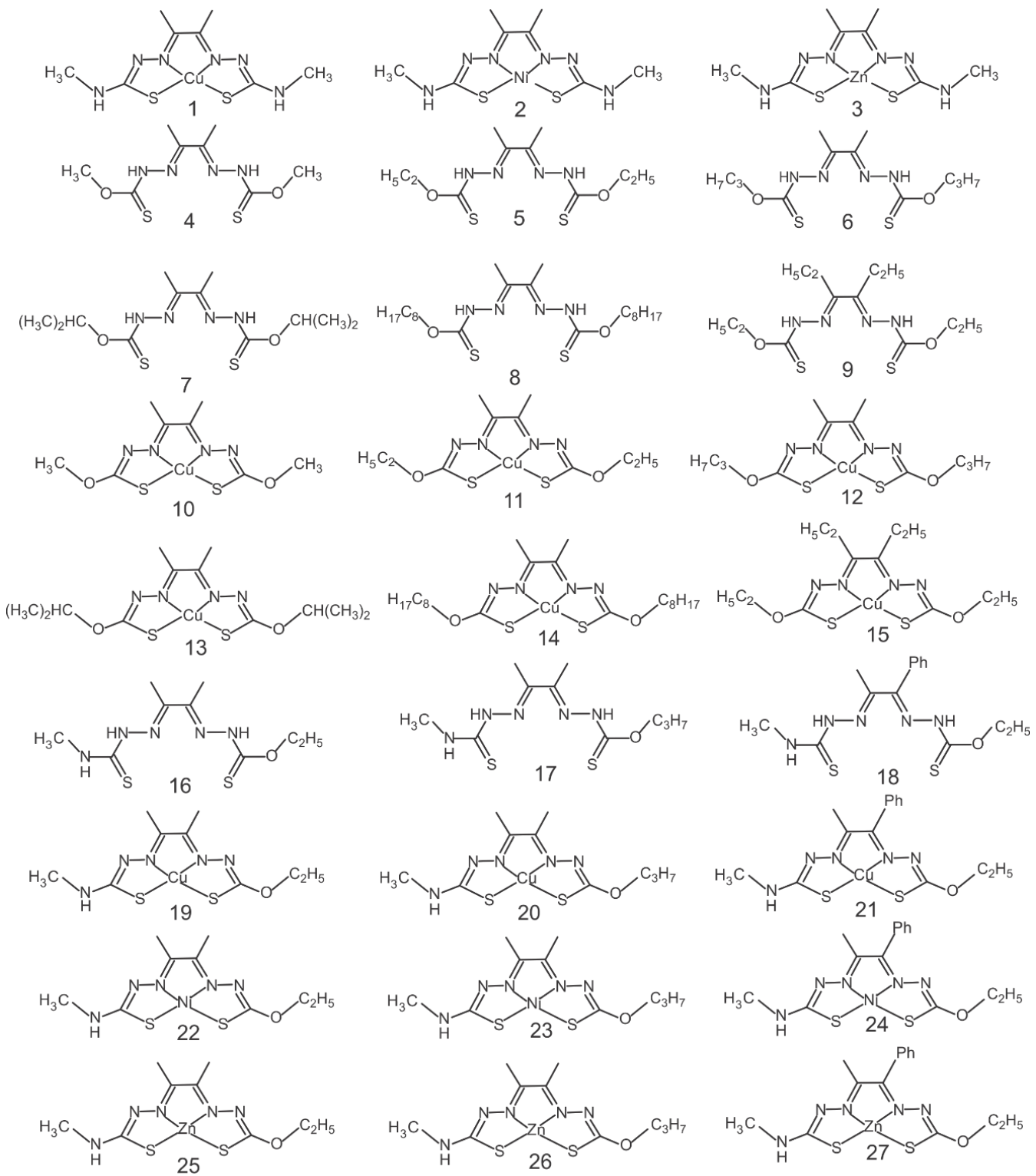<smiles>CC1=C(C)N2N=C(N(C)C)C[N@@+]23C=C1N=C(N(C)C)C3</smiles><smiles></smiles>

Figure 1-1. Numbering scheme for all complexes. 
Chapter 1 provides an overview of the dissertation and introduces previous implementations of metal BTSC complexes in diagnostic imaging, anti-cancer therapeutics, and homogeneous hydrogen evolution catalysis. Chapter 2 specifies the experimental conditions, such as synthetic methodologies, yields, and characterization techniques for the complexes in this dissertation. This chapter also describes conditions used to determine electrochemical data, homogeneous catalysis conditions, and heterogeneous catalysis characterization. Chapter 3 describes the syntheses and characterization of a new class of $\mathrm{N}_{2} \mathrm{~S}_{2}$ chelates related to BTSCs and their $\mathrm{Cu}(\mathrm{II})$ complexes. The six new N,N'-bis(alkylthiocarbamate)butane-2,3-diimine chelates incorporate alkoxy alkyl chains derived from primary and secondary alcohols in place of the extensively studied pendant amines. The reduction potential of the $\mathrm{Cu}(\mathrm{II})$ complexes is tuned through the incorporation of the alkoxy functional group while maintaining the structural integrity that confers biological effects of BTSCs. Crucially, the data learned from this system allows for greater modulation of the ligand framework to tune the redox chemistry for either HER or anti-cancer. Chapter 4 focuses on the immobilization of three nickel (II) BSTC complexes on the glassy carbon electrodes as heterogeneous HER catalysts. These studies revealed a high dependence on surface morphology and solid state interactions that led to an improved electrode contact to reduce overpotential. Further, these nickel (II) BTSC complexes were not covalently linked to the electrode surface which allowed for dynamic reorganization on the electrode surface. Despite not being covalently linked, the complexes displayed robust activity over extended cycling. In Chapter, 5 the anti-cancer activity of new asymmetric chelates related to BTSCs and their metal complexes are discussed. It was observed from the MTT assays and electrochemistry that 
there is a clear effect of structure on the compound's anti-cancer activity. The reduction potentials illuminated features that conferred the selectivity for cancer cells compared to the healthy cells. Lastly, Chapter 6 contains concluding thoughts and future directions to the research in Chapters $3-5$.

\subsection{Synthesis of BSTCs and Radioimaging Applications}

A class of compounds that has been applied in varying fields are thiosemicarbazides, $\mathrm{RNHCSNHNH}_{2}$. The thiosemicarbazide family has been well studied in areas such as tuberculosis treatments, ${ }^{1-2}$ anti-cancer activity, ${ }^{3-5}$ and metal absorbents. ${ }^{6}$ These wide ranging abilities of the thiosemicarbazide family stem from its modular synthesis, ${ }^{7}$ which enables alteration of the molecule to suit the needs of a given application. Furthermore, the hydrazine functionality of the thiosemicarbazide is capable of undergoing a condensation reaction with a dione to generate bis-thiosemicarbazones (BTSC). ${ }^{8-9}$ The identity of the dione used can be varied to generate an extensive library of ligands that chelate a vast variety of metals. $^{8,10-21}$

Like their thiosemicarbazide precursors, BTSCs have been extensively studied for applications across multiple disciplines of science, such as the treatment of neurodegenerative diseases, ${ }^{22-26}$ selective imaging of hypoxia, ${ }^{21,27-33}$ and as catalysts for the hydrogen evolution reaction (HER). ${ }^{10,12,34-35}$ The overarching similarity in these various applications is the accessibility of the reduction potential of the metal complex. ${ }^{16}$ For example, the HER requires a catalyst for the reduction of protons and hypoxia imaging relies on a complex that changes oxidation state under low oxygen conditions, where cells inherently are in a more reducing environment than normoxic conditions. Thus the ability to tune the reduction potential of a compound is paramount to the success in these fields. 
Overall the synthetic methodology for BTSCs involves the condensation of a thiosemicarbazide with a dione. The manner of the condensation can be either symmetric, Figure 1-2A, or non-symmetric, Figure 1-2B. The symmetric condensation maintains equivalent $\mathrm{R}$ groups from the thiosemicarbazide, but allows for variation in the dione $\mathrm{R}$ groups. The prototypical example of this is pyruvaldehyde bis(N4-methylthiosemicarbazonato) $\left(\mathrm{H}_{2} \mathrm{PTSM}\right) .{ }^{36-37}$ The non-symmetric condensation route allows for a finer tuning technique of either solubility, redox potential, or structure effects. For example, Que et al. ${ }^{32}$ exploited this methodology to attach a $-\mathrm{CH}_{2} \mathrm{CF}_{3}$ functionality for enhanced selectivity in cancer cell imaging. ${ }^{32}$

A<smiles>[R]NC(=S)NN=C([R])C([R])=NNC(=S)N[R7]</smiles>

B<smiles>[R]NC(=S)NN=C([R])C([R])=O</smiles><smiles>[R]NC(=S)NN=C([R])/C([R])=N\NC(=S)N[R]</smiles>

Figure 1-2. Modular synthesis of symmetric condensation (A) and asymmetric condensation (B) of BTSCs 
The modulation of reduction potential through variation of the BTSC ligand framework is exemplified by comparison of CuATSM and glyoxal bis(N4-methylthiosemicarbazonato) copper(II) (CuGTSM), Figure 1-3. The only structural difference between these two complexes is the backbone moiety in which CuATSM has a 2,3butylene bridge whereas CuGTSM has an ethylene linker. The difference in electron donor ability of these substituents has a dramatic effect on the reduction potential. The $\mathrm{Cu}^{\mathrm{II} / \mathrm{I}}$ couple for CuATSM occurs at $-1.11 \mathrm{~V}$ vs. ferrocenium/ferrocene $\left(\mathrm{Fc}^{+} / \mathrm{Fc}^{0}\right)$, whereas the $\mathrm{Cu}^{\mathrm{II} / \mathrm{I}}$ potential for CuGTSM is observed $160 \mathrm{mV}$ more anodic at $-0.95 \mathrm{~V} .{ }^{38}$ This difference in potential is the reason for the different biological activity of these two compounds. Both CuATSM and CuGTSM have been evaluated for hypoxia imaging via radiolabeling with either ${ }^{64} \mathrm{Cu}$ or ${ }^{62} \mathrm{Cu}$. The more difficult to reduce CuATSM is found to be selectively retained in only hypoxic cells. The retention is based on the generation of a charged complex that only occurs in hypoxic cell, which prevents diffusion of the $\mathrm{Cu}$ from the cell. ${ }^{30}$ However, the more easily reduced CuGTSM, is reduced and retained in both normoxic and hypoxic cells. ${ }^{30}$ This finding has led to the synthesis of a variety of derivatives in efforts to enhance the selectivity for hypoxia imaging. The majority of these compounds focused on functionalizing the pendant amine to enhance imaging.

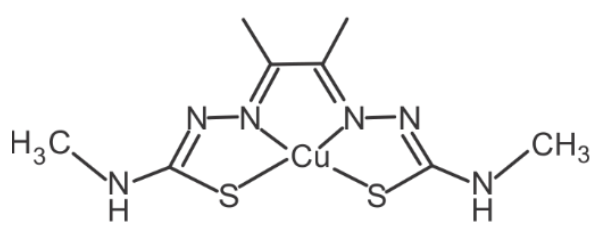

CUATSM

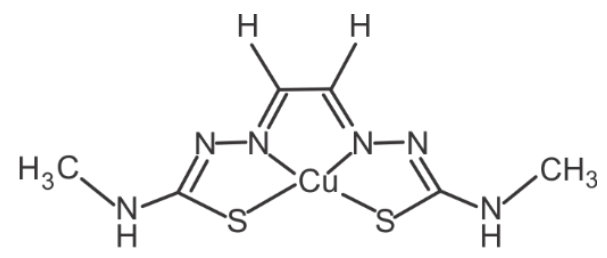

CUGTSM

Figure 1-3. Structures of CuATSM (left) and CuGTSM (right). 
As described above, the general synthetic methodology for BTSCs employs a condensation of a thiosemicarbazide with a dione. The desired thiosemicarbazide can be synthesized from the corresponding isothiocyanate and hydrazine. ${ }^{7}$ This synthetic route lends itself for various amine derivatives to be incorporated into the ligand framework. The modifications to the ligand framework are focused on tuning reduction potential or enhancement of radioimaging. Modifications to increase uptake of the radiolabeled compound include incorporation of the octa-peptide, octreotide, to the ligand framework. ${ }^{33}$ Modifications to tune the reduction potential of the complex include incorporation of an alyll functionalitiy ${ }^{15}$ or a pyrollidine. ${ }^{39}$ Transamination by using 4,4-dimethyl-3thiosmeicarbazide can be employed to further modify the ligand framework by displacing the tertiary amine with the desired primary amine. This route has been particularly useful in the development of non-radiolabeled active imaging molecules. ${ }^{40}$ Using this route Emily Que and co-workers ${ }^{32}$ notably incorporated a $-\mathrm{CH}_{2} \mathrm{CF}_{3}$ functionality off one or both pendant amines that led to $\mathrm{Cu}$ complexes with an anodic shift of $70 \mathrm{mV}$ per $-\mathrm{CH}_{2} \mathrm{CF}_{3}$ group. Upon entering a hypoxic environment, the mono $-\mathrm{CH}_{2} \mathrm{CF}_{3}$ substituted $\mathrm{CuATSM}$ derivative was reduced by one electron from a paramagnetic, $d^{9} \mathrm{Cu}^{\mathrm{II}}$ to a diamagnetic, $\mathrm{d}^{10} \mathrm{Cu}^{\mathrm{I}}$. The latter compounds can be readily observed by ${ }^{19} \mathrm{~F}$ MRI due to the absence of paramagnetic broadening that render the $\mathrm{Cu}^{2+}$ complexes unobservable. Additionally, the kinetic lability of $\mathrm{Cu}^{\mathrm{I}}$ promotes dissociation from the ligand framework adding to the image enhancement. The $\mathrm{Cu}^{\mathrm{II} / \mathrm{I}}$ potential for the $\mathrm{di}-\mathrm{CH}_{2} \mathrm{CF}_{3}$ substituted $\mathrm{CuATSM}$ derivative is similar to that of CuGTSM and it is too anodic for application in hypoxia imaging. The more anodic reduction potential of CuGTSM and related complexes makes them attractive candidates for other applications including cancer therapeutics. 


\subsection{Cancer and BTSCs}

Recently, both CuGTSM and CuATSM were evaluated for their activity against prostate cancer cells. ${ }^{38}$ CuGTSM displayed a lower LD50 $(1.5 \mu \mathrm{M})$ than CuATSM (7.0 $\mu \mathrm{M})$ for the treatment of PC-3 cells. The activity of CuGTSM was attributed to an increase of bioavailable copper, which induces apoptosis much like the related 2-keto-3-ethoxybutyraldehydebis(thiosemicarbazonato) (KTS) $\mathrm{Cu}(\mathrm{II})$ complex. ${ }^{41}$ To examine this, the cell culture was treated with the known copper chelator tetrathiomolybdate (TTM). The chelator affected the potency of CuGTSM, but that of CuATSM remained unchanged. This result was confirmed by using a metal responsive element (MRE)-luciferase reporter. ${ }^{42-43}$

Although in vitro studies provide crucial insight to potential therapies, application in vivo is crucial for the further development of a therapy. Using the TRAMP transgenic prostate cancer mouse model, ${ }^{44}$ both CuGTSM and CuATSM were investigated for their viability in vivo. Weighing of the genitourinary tracts showed a $70 \%$ reduction in tumor weight relative to the control when the mice were treated with CuGTSM. Further evaluation using the Suttie scoring system ${ }^{45}$ showed marked improvement with the CuGTSM treatment. It should be noted that this treatment was not without side effects. Mice treated with CuGTSM suffered from kidney toxicity caused by copper accumulation.

Pharmokinetic experiments determined that the chelated copper does not exchange with other metals in vivo. ${ }^{38}$ Further, ZnGTSM was examined as a potential anti-cancer agent, but was found to be inactive in agreement with previous reports. ${ }^{41,46}$ It was previously established that the copper ionophores clioquinol and disulfiram inhibit proteasomal chymotrypsin-like activity. ${ }^{47-49}$ To examine if CuGTSM exhibited similar 
effects, the expression and subcellular localization of the X-linked inhibitor of apoptosis protein (XIAP) was monitored. ${ }^{49-51}$ Inhibition of proteasomal activity causes the localization of XIAP to move from the cytoplasm to the nucleus. Following treatment with CuGTSM, the XIAP was found to have been localized in the nucleus, confirming the inhibitions of proteasomal activity by CuGTSM. This result of XIAP localization in the nucleus was not observed following treatment with CuATSM.

The previous work done on CuGTSM by Cater et al. ${ }^{38}$ prompted the exploration for new copper BTSCs with more anodic redox couples than CuATSM for therapeutic purposes. Palanimuthu et al. ${ }^{39}$ focused on the incorporation of cyclic moieties into the pendant amine. The cyclic moieties were synthesized by the condensation of 4pyrrolidinyl-3-thiosemicarbazide with benzil or butanedione or the condensation of 4methyl-4-phenylthiosemicarbazide with glyoxal. These compounds were then evaluated for anti-cancer activity using MTT assays of the carcinogenic cell lines SiHa (cervical cancer), MCF-7 (breast cancer), PC-3 (prostate cancer), A-2780 (ovarian cancer), and HepG2 (hepatocellular liver cancer). The results from the MTT assays showed CuGTSC as the most potent compound. The $\mathrm{Cu}^{\mathrm{II} / \mathrm{I}}$ couple of $\mathrm{CuGTSC}$ was found to be $400 \mathrm{mV}$ more anodic than CuATSM. The compound obtained from condensation of 4-pyrrolidinyl-3thiosemicarbazide with benzyl had a $\mathrm{Cu}^{\mathrm{II} / \mathrm{I}}$ couple $240 \mathrm{mV}$ more anodic than CuATSM but displayed no effect on carcinogenic cell lines other than HepG2. This result indicates that reduction potential is not the only determinant in the anti-cancer activity.

To confirm cellular uptake and location of copper from CuGTSC and CuGSTC $\cdot \mathrm{HCl}$ in to the cancer cells, ICP-OES was employed by Palanimuthu and co-workers to show that the copper was preferentially located in the cytoplasm rather than the nucleus after 
treatment with $\mathrm{CuGTSC}$ or $\mathrm{CuGSTC} \cdot \mathrm{HCl}$. The protonated complex, $\mathrm{CuGSTC} \cdot \mathrm{HCl}$, is expected to equilibrate with the buffer during evaluation to generate CuGTSC in situ. It remains unclear as to why the observed results for both neutral and protonated differ. Further measurement of the lipophilicity of CuGTSC and CuGSTC $\cdot \mathrm{HCl}$ was conducted by a modification of the common water and 1-octanol partition coefficient study. ${ }^{52}$ Chloroform was employed in place of octanol due to solubility issues and the conversion factor $\mathrm{P}_{\text {octanol }}=\left(1.343+\log \mathrm{P}_{\text {chloroform }}\right) / 1.126 .{ }^{53}$ The measurements revealed a correlation of cytotoxicity with their lipophilicity, with the more lipophilic CuGTSC requiring a lower does to achieve cytotoxicity. The compounds were then investigated to examine the mechanism of apoptosis induction.

Several previous reports observed that CuBTSCs were capable of inhibiting DNA synthesis. ${ }^{54-57}$ This is in contrast to the reactive oxygen species (ROS) mediated anti-cancer activity of the mono-TSCs. ${ }^{54,58}$ Using the $\left[{ }^{3} \mathrm{H}\right]$ thymidine incorporation assay, it was found CuGTSC (Figure 1-4) did inhibit DNA synthesis. Further, in a competition with ethidium bromide, the binding affinities of $\mathrm{CuGTSC}$ and $\mathrm{CuGSTC} \cdot \mathrm{HCl}$ for DNA were determined. These results showed that $\mathrm{CuGSTC} \cdot \mathrm{HCl}$ (i.e., charged species) displayed a higher binding affinity for DNA than the neutral CuGTSC, indicating an electrostatic component to the interaction. Lastly, the results from viscosity experiments pointed to intercalation as the mode of binding. 


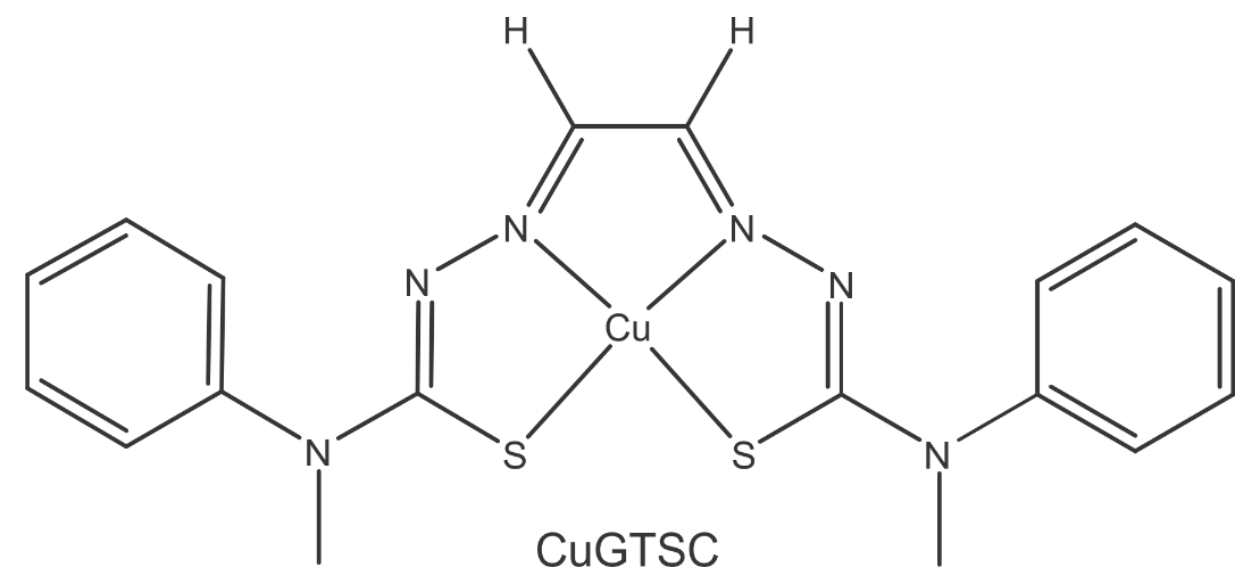

Figure 1-4. CuGTSC structure.

In attempt to elucidate the mechanism related to inhibition of DNA synthesis, DNA cleavage and topoisomerase inhibition ${ }^{59-62}$ studies were performed. Using supercoiled pBR322 plasmid DNA and gel electrophoresis, the amount of cleaved DNA could be measured. A Human Topo II $\alpha$ kit was employed to measure the inhibition of topoisomerase. The majority of the BTSC $\mathrm{Cu}$ complexes investigated did not display a large amount of cleavage or inhibition, including CuGTSC. The protonated CuGSTC $\cdot \mathrm{HCl}$ derivative however, showed $86 \%$ cleavage and $90 \%$ inhibition, significantly higher than CuGTSC which had values of $24 \%$ and $52 \%$, respectively. Further the cleavage of DNA was not mediated by ROS since the same result was found when run under in argon atmosphere.

The interesting result of the structure activity relationship displayed by Palanimuthu was advanced by Palma and co-workers. ${ }^{63}$ Incorporating cyclic amines as the pendant amine of the BTSC resulted in reduction potential closer to CuATSM than CuGTSM. While this modification led to enhanced cytotoxicity, there was decreased selectivity for carcinogenic versus healthy cells. The observed increase in cytotoxicity was 
attributed to the increased lipophilicity conferred by the cyclic amine increasing cellular uptake relative to CuATSM. Although cellular uptake was increased, the efflux rate of the compound with cyclic amines remained unchanged compared to CuATSM.${ }^{64}$ It should be noted that the presence of a pendant secondary amine may lead to efflux via multidrug resistant protein one (MDR-1) for BTSC complexes. ${ }^{65}$

For anti-cancer activity with BTSCs the metal employed has generally been copper. Recently, a series of cobalt(III) containing BTSCs ${ }^{11}$ was evaluated for their anti-cancer properties. As observed with $\mathrm{Cu}$, the easier to reduce species were found to be the most potent. However, this class of compounds displayed relatively poor activity. Interestingly, when copper was scrubbed from culture medium with tetrathiomolybdate, ${ }^{66-68}$ the anticancer activity was further diminished, indicating a copper dependence. Further studies have not been performed to elucidate if this result is an effect of metal exchange or other $\mathrm{Cu}$-mediated processes.

\subsection{Hydrogen Evolution and BTSCs}

Whereas cancer applications of BTSCs focus primarily on modulation of the reduction potential, HER catalysts require an understanding of ligand basicity. The US

energy economy is largely been based on non-renewable resources, Figure $1-5 .{ }^{69}$ A major drawback of renewable energy sources such as solar and wind is the need to store energy for peak usage. The use of $\mathrm{H}_{2}$ as an energy carrier is considered green as the combustion of $\mathrm{H}_{2}$ produces water rather than greenhouse gasses as a product. However, for a whole process to be considered green, the production of $\mathrm{H}_{2}$ also needs to be ecologically friendly. The two industrially employed techniques of $\mathrm{H}_{2}$ production are steam methane reformation and coal gasification, both of which generate $\mathrm{CO}_{2}$ as a byproduct. The cleanest way to 
produce hydrogen is by splitting water. Crucially, the reaction is non-spontaneous and requires a thermodynamic potential of $1.23 \mathrm{~V}$. However, any system employed will have to supply additional energy to overcome the overpotential. Overpotential results from an activation barrier due to electron transfer barriers and diffusion effects. The ideal catalyst will operate at a minimal overpotential and be capable of economic scale up.

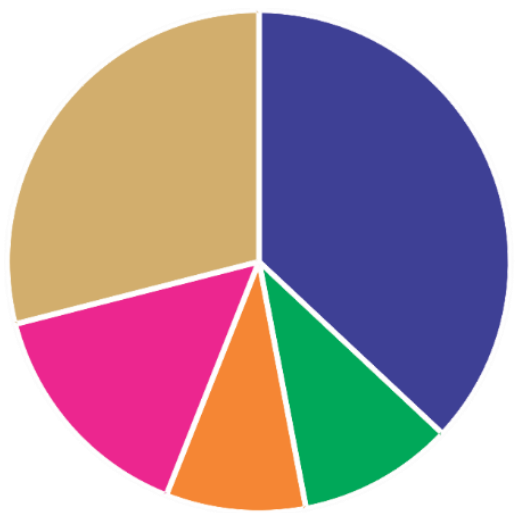

- Petroleum (37\%)

Renewable (10\%)

Nuclear (9\%)

- Coal $(15 \%)$

- Natural gas (29\%)

Figure 1-5. Distribution of energy consumption in the US. ${ }^{69}$

Recently Haddad et al. evaluated the $\mathrm{H}_{2}$ ATSM ligand and ZnATSM as homogeneous HER catalysts. ${ }^{10}$ This was the first evaluation of BTSCs as possible HER catalysts and metal-free HER catalysis. The Zn complex was a viable HER catalysts in both acetonitrile and methanol, whereas the ligand was active only in methanol. The cyclic voltammogram of $\mathrm{H}_{2} \mathrm{ATSM}$ displayed one irreversible reduction at $-2.1 \mathrm{~V} \mathrm{vs} \mathrm{Fc}^{+} / \mathrm{Fc}$ in the absence of acid. Titration of acetic acid to a $3 \mathrm{mM}$ ligand solution lead to an increase of current at $-2.1 \mathrm{~V}$ until the acid reached $9.8 \mathrm{mM}$, at which the current plateaued. The turnover frequency (TOF) from the ligand to produce hydrogen was $1320 \mathrm{~s}^{-1}$ with an overpotential of $1.43 \mathrm{~V}$. 
The $\mathrm{Zn}$ complex performed better compared to the ligand in methanol. It should be noted that the $\mathrm{Zn}$ complex displayed no reduction events in the absence of acid in the cyclic voltammogram. Upon addition of acid, a catalytic peak was observed at $-1.7 \mathrm{~V}$, implying the first step of HER with ZnATSM is protonation. Acetic acid was added up to $12.0 \mathrm{mM}$, upon which the increase in current plateaued. Under these conditions, the TOF was found to be $1170 \mathrm{~s}^{-1}$, slightly lower than the free ligand. However, the overpotential of the $\mathrm{Zn}$ derivative in methanol was $0.756 \mathrm{~V}$, which is $0.674 \mathrm{~V}$ lower than with the ligand alone. With an identical environment, a bulk electrolysis over the course of 2.5 hours gave a turnover number (TON) of 37 with a Faradaic efficiency of $85 \%$. Interestingly in acetonitrile, the acid plateau occurred at $23 \mathrm{mM}$, giving an order of magnitude larger TOF of $11,700 \mathrm{~s}^{-1}$ but with a larger overpotential of $1.074 \mathrm{~V}$. This large difference could potentially arise from a change in the properties of the solvents after addition of acid such as the dielectric constant. No studies were performed to measure the change of the physical properties of the solvents. The catalytic event was found to be at $-2.3 \mathrm{~V}$ which is close to the free ligand reduction even without acid.

The mechanistic studies revealed that the reaction is first order with respect to both ZnATSM and acetic acid. ${ }^{70}$ Kinetic isotope effect (KIE) experiments ${ }^{71-73}$ in acetonitrile, using deuterated acetic acid gave a value of 1.2 , which is unique from those previously reported for HER electrocatalysts, insinuating an unprecedented mechanism of action. Combining simulations and density functional theory (DFT) calculations, two plausible mechanisms were determined. It is crucial to note that both pathways involve ligand centered radical coupling, require protonation as the first step, and require the cooperation of two complexes, Scheme 1-1. 


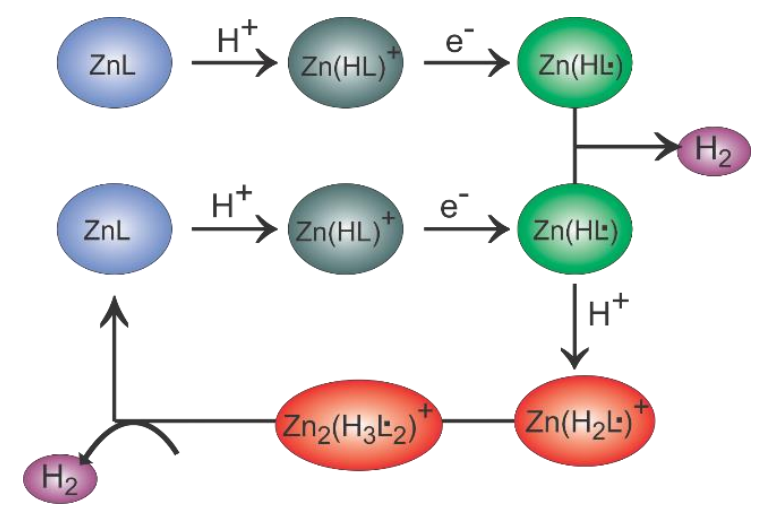

Scheme 1-1. ZnATSM possible HER mechanisms.

In an effort to improve the performance of the ATSM framework as a HER catalyst, Haddad et al. evalutaed CuATSM. ${ }^{34}$ The hypothesis being that the metal center could assist in the ligand reactivity. Prior to any acid additions a reversible reduction was observed at $-1.20 \mathrm{~V} \mathrm{vs} \mathrm{Fc}^{+} / \mathrm{Fc}^{0}$ in acetonitrile, which is assigned to the $\mathrm{Cu}^{\mathrm{II} / \mathrm{I}}$ couple. Addition of acid led to an anodic shift of this event to $-0.95 \mathrm{~V}$, indicative of protonation prior to catalysis. This was later confirmed via spectrophotometric titrations and simulations. Addition of acid also led to a new cathodic peak at $-1.70 \mathrm{~V}$. Interestingly, at lower acid concentrations, the mechanism is second order with respect to acid. However, as concentrations increase, it becomes independent of acid concentration at $0.269 \mathrm{M}$. This change in acid dependency is indicative of a pre-equilibrium step prior to $\mathrm{H}_{2}$ release. The TOF for this system was found to be $10,000 \mathrm{~s}^{-1}$, with an overpotential $0.8 \mathrm{~V}$, which is $0.3 \mathrm{~V}$ lower than ZnATSM. ${ }^{10}$ Identical studies were performed in DMF, however the catalytic activity was significantly diminished as compared to studies in acetonitrile. It is hypothesized that this is due to the strong hydrogen bonding nature of DMF. KIE experiments gave a value of 7.54 which is consistent with that observed for a ligand centered rhenium thiolate HER catalyst. ${ }^{74}$ Further, it was revealed that one proton was involved in the rate determining step. From 
the combination of experimental results, digital simulations of cyclic voltammogram (CV) data, and DFT calculations a mechanism was proposed, Scheme 1-2. Each step of the mechanism was confirmed by DFT to be the lowest energy state. While both $\mathrm{Zn}$ and CuATSM undergo a CECE mechanism, chemical protonation (C) followed by reduction (E), the copper proceeds through a monomeric pathway in contrast with the zinc. Another difference between these two systems was the development of a film on the working electrode following bulk electrolysis for the copper derivative but not for the zinc. Thus, it is impossible to rule out any heterogeneous effects of HER catalysis for the copper complex.

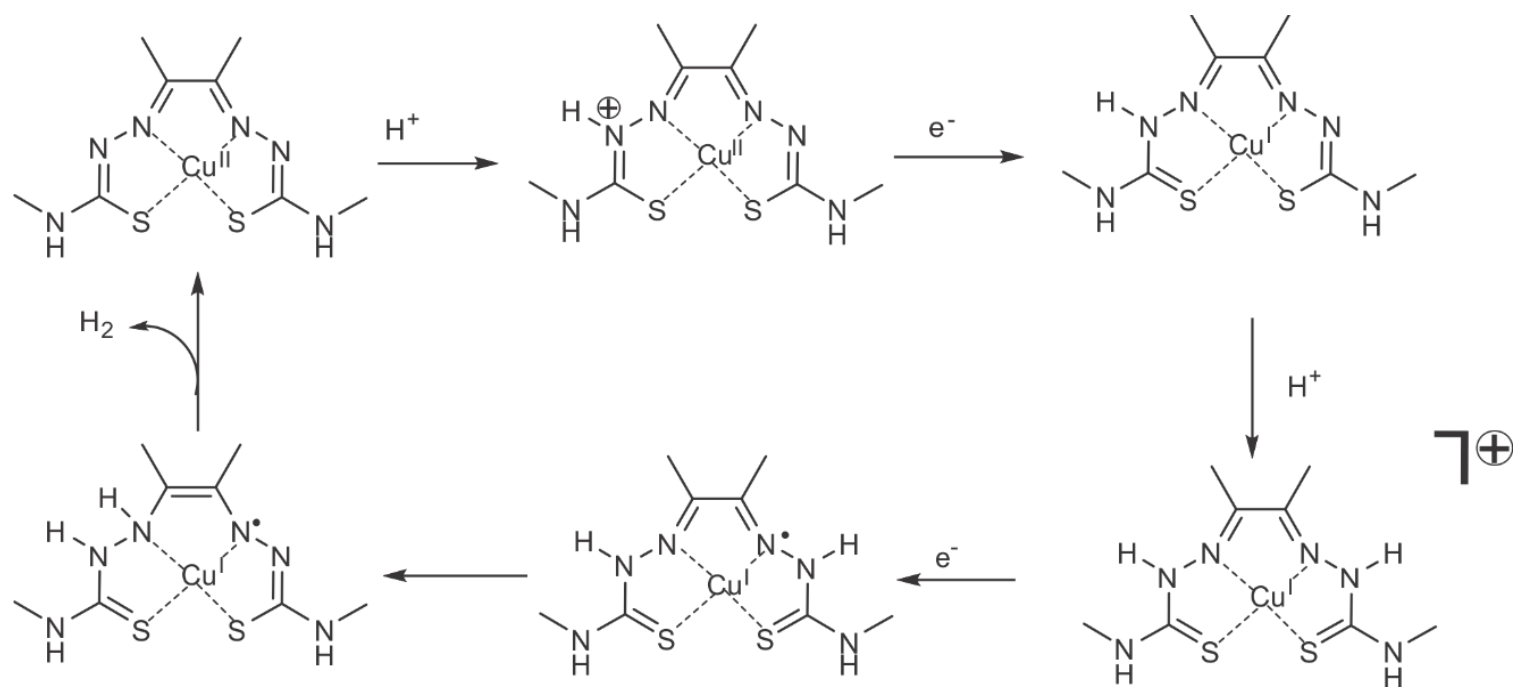

Scheme 1-2. Mechanism for $\mathrm{H}_{2}$ evolution from CuATSM.

With a wide variety of ligands available, Straistari and co-workers synthesized and studied the HER catalysis of a NiATSM derivative. ${ }^{35}$ This particular derivative replaced the methyl off the pendant amine with para-anisole, Figure 1-6. This was then used to chelate nickel giving a complex with reduction events at -1.57 and $-2.10 \mathrm{~V}$ in DMF. With the addition of trifluoroacetic acid, a new reduction event occurred at $-1.23 \mathrm{~V}$. This was 
attributed to HER catalysis and reached acid saturation at $100 \mathrm{mM}$. Using foot-of-the-wave analysis, a TOF was calculate to be $3080 \mathrm{~s}^{-1}$.

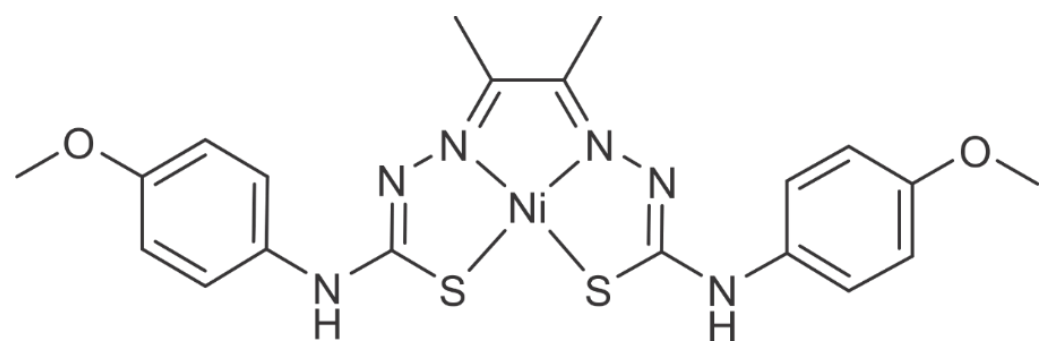

Figure 1-6. Structure of NiATSM para-anisole.

Bulk electrolysis at $-1.73 \mathrm{~V}$ gave a TON of 21 and a Faradaic efficiency of $80 \%$. Interestingly, control experiments did not reveal any formation of a film or nanoparticles during the course of the bulk electrolysis. Further mechanistic studies were carried out using DFT computations. The first reduction was assigned as ligand centered and the subsequent reduction was found to be metal centered. When a proton is added after the first reduction, the site of protonation was found to be the imino nitrogen of the ligand framework. Reduction after this protonation remained on the nickel, as previously established in the absence of acid. $\mathrm{A} \mathrm{Ni}{ }^{\mathrm{III}}-\mathrm{H}$ hydride is produced after the second protonation event prior to $\mathrm{H}_{2}$ release. This novel mechanism was the first to use the metal center directly to evolve $\mathrm{H}_{2}$ for the BTSC class of compounds. The overpotential of this novel NiBTSC system was lower than the previously reported ATSM derivatives studied by Haddad et al. ${ }^{10,34}$

The results from Straistari et al. ${ }^{35}$ led Jain et al. to evaluate the electrocatalytic behavior of NiATSM. ${ }^{75}$ The cyclic voltammograms displayed two reversible reductions at -1.83 and $-2.45 \mathrm{vs} \mathrm{Fc}^{+} / \mathrm{Fc}$ in acetonitrile. These values for NiATSM are cathodically shifted to those of the para-anisole derivative reported by Straistari et al. ${ }^{35}$ Upon addition of acetic 
acid, a catalytic peak was observed at $-2.35 \mathrm{~V}$ and reached plateau after the addition of 12.6 $\mathrm{mM}$. This corresponded to a TOF of $4,200 \mathrm{~s}^{-1}$, an overpotential of 0.53 and a TON of 48 after 4 hours. Trifluoroacetic acid was also used as an acid, while there was an anodic shift in the catalytic peak to $-2.26 \mathrm{~V}$, the overall performance diminished. The corresponding TOF, overpotential, and TON were $1,300 \mathrm{~s}^{-1}, 0.67 \mathrm{~V}$ and 24 . The same trend was observed when DMF was used as a solvent. Overall, the performance of the catalyst in DMF was lower. As previously mentioned this is caused by the hydrogen bonding of DMF.

Mechanistic studies were performed on NiATSM, including concentration dependence studies, which revealed the reaction is first order with respect to the catalyst. There was no evidence of the formation of a film or nanoparticles. Spectrophotometric titrations displayed no protonation of the complex without prior reduction, in agreement with previous reports. The experimentally determined KIE value was inverse 1 , which is consistent with the formation of a metal hydride. Combining these experimental results with DFT computations a mechanism was postulated. A ligand centered reduction occurs prior to protonation at the hydrazine nitrogen. The second reduction occurs on the metal center resulting in a metal hydride. This hydride intermediate is then capable of reacting with a proton in solution to evolve $\mathrm{H}_{2}$. Overall, the mechanism is ECEC. The mechanism is slightly different from that proposed by Straistari et al. for the para-anisole nickel complex (Figure1-6) as the first protonation occurs on the hydrazino nitrogen for NiATSM, whereas it is reported to occur on the imino nitrogen by Straistari et al..$^{35}$

Straistari and co-workers continued to examine the effect of the chelated metal on overall performance of the catalyst. ${ }^{12}$ The same para-anisole BSTC ligand was used to prepare the palladium complex. This is the first example of a later row transition metal 
being employed with the BTSC ligand framework as a HER catalyst, however still remaining in the same family as nickel. Two reduction events are observed in the cyclic voltammogram in DMF, at potentials similar to the nickel derivative at -1.55 and $-2.07 \mathrm{~V}$. Following the reported methods for the nickel complex the centers of the reduction were determined using DFT. The reductive events were again found to first occur on the ligand and then on the metal, like the previous nickel systems. ${ }^{35,76}$ These results were further corroborated by calculation of the reduction potentials.

The palladium system was then evaluated for its viability as a HER catalyst. Trifluoroacetic acid was added until saturation occurred at $100 \mathrm{mM}$. As the previously described nickel system is proposed to operate via ECEC, so is the palladium. ${ }^{35,76}$ DFT calculations were carried out to confirm this, along with spectrophotometric titrations that showed that the complex did not protonate upon the addition of trifluoroacetic acid without first undergoing a reduction. Interestingly, the first protonation event, while still ligand centered was found to occur on the hydrazino nitrogen as in CuATSM. The full HER mechanism is much like that of CuATSM involving the radical coupling of two ligand centered hydrogens, Scheme 1-2.

A drawback of homogenous catalysis is the lack of long term stability and relatively large overpotentials. Heterogeneous catalysts generally offer a longer lifetime, but current ones are more expensive as they generally employ precious metals. The current standard for heterogeneous HER catalysis is a platinum electrode which operates at nearly no overpotential. While a multitude of work has been done on developing more cost efficient heterogeneous catalysts, there has yet to be any implementation of BTSCs on an electrode surface. As described earlier, CuATSM even under homogeneous conditions generated a 
film on the electrode surface. Zhang et al. expanded upon this by drop-casting various BTSCs on a glassy carbon surface. ${ }^{77}$

The ligand frameworks that were examined were ATSM and ATSP (Figure 1-7) as well as their copper and zinc chelates. Using scanning electron microscopy (SEM), all catalysts were evaluated before and after reductive cycling. It was apparent in all cases that after reductive cycling the film from the deposited catalyst was roughened by the evolution of hydrogen. In the cases of the ligand this meant a uniform film became reorganized and in the metal complexes, microcrystals became more amorphous.

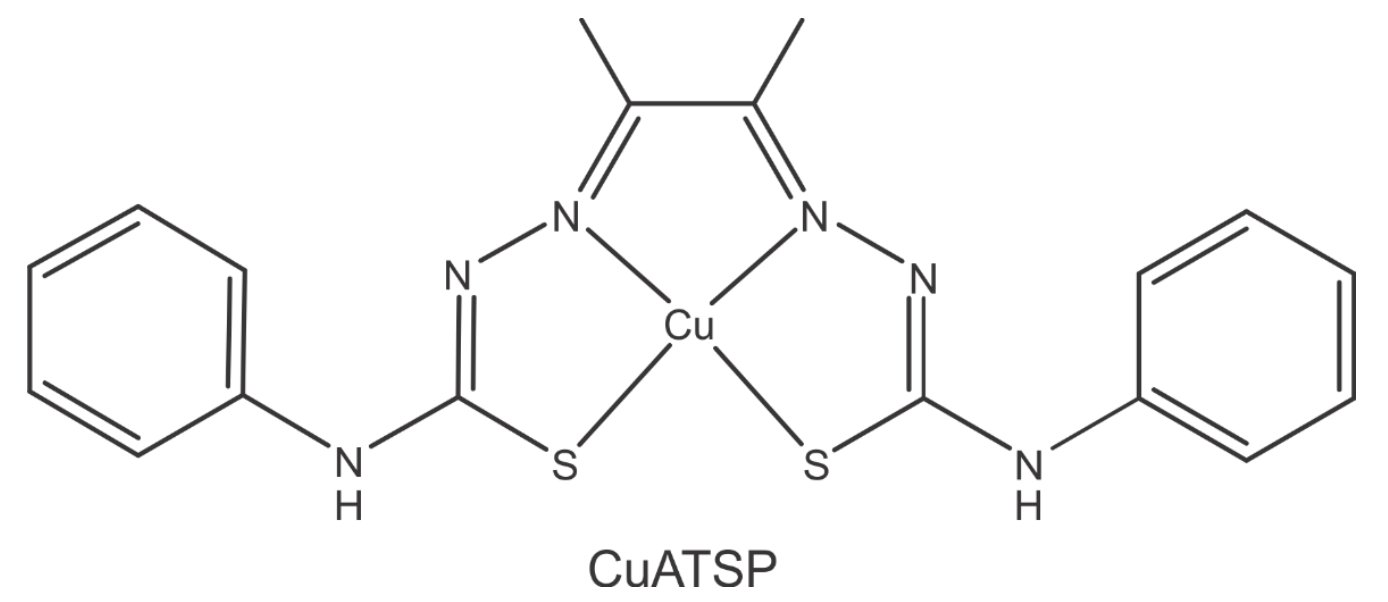

Figure 1-7. Structure of CuATSP.

To confirm stability of the film after deposition on the working electrode, XPS was employed. For both frameworks, the ligand and copper complex were found to remain intact on the electrode surface. The zinc derivatives however were found to demetallate during the reductive cycling; the ligand remained intact though. This was also confirmed by UV-visible spectroscopy, in which after reductive cycling the zinc compounds electronic spectra were identical to that of the free ligand indicating they were no longer zinc chelates. 
It should be noted that various loading amounts were required to achieve optimum performance for each system. Overall the best system was found to be copper derivatives, with an overpotential of $0.774 \mathrm{~V}$ and $0.745 \mathrm{~V}$ for CuATSM and CuATSP, respectively. While this is not a marked improvement from the homogeneous system of the $0.8 \mathrm{~V}$ overpotential for CuATSM, ${ }^{34}$ the immobilization of these compounds on the electrode surface displayed longevity of over 25 hours. One technique that has yet to be fully exploited is the generation of carbon paste electrodes with BTSCs. Zhang et al. showed that the synthesis of such an electrode with CuATSM led to a dramatic decrease in the overpotential to $0.531 \mathrm{~V}$.

\subsection{Summary}

Overall BTSCs represent a versatile class of ligands suitable for numerous applications. The synthetic methodology lends itself to be varied in a multitude of locations to generate an extensive library of compounds. This modular synthesis dovetails with the need to fine tune the redox activity for the diverse applications in which these systems are employed. Applications that exploit the redox activity and ease of synthesis include hydrogen evolution catalysis, radioimaging, and therapeutics. In this dissertation, the library of BTSCs and structurally related derivatives, including a hybrid system, are expanded and explored for their use in heterogeneous HER and anti-cancer activity. 


\section{CHAPTER II \\ EXPERIMENTAL}

\subsection{Physical Methods}

Elemental analyses were performed by Midwest Microlab (Indianapolis, IN). The electronic spectra were measured in quartz cuvettes $(1 \mathrm{~cm}$ pathlength) using an Agilent 8453 diode array spectrometer. Mass spectrometry (ESI-MS, positive mode) was performed by the Regenerative Medicine Research Center at the West China Hospital, Sichuan University, Chengdu, China or the Laboratory for Biological Mass Spectrometry at Texas A\&M University, or MALDI-TOF at the University of Louisville. X-band electronic paramagnetic resonance (EPR) spectra were collected on powder samples at room temperature using a Bruker EMX (Bruker, Billerica, MA) spectrometer and simulated using EasySpin (MATLAB, version 5.2.23). ${ }^{78}$ The infrared spectra were recorded on a Thermo Nicolet 360 FT-IR spectrophotometer with ATR attachment (Thermo Fisher, Waltham, MA). NMR spectra were obtained on Varian $400 \mathrm{MHz}$ spectrometer at $25{ }^{\circ} \mathrm{C}$. X-ray crystallographic studies were performed by Dr. Mark Mashuta at the Department of Chemistry, University of Louisville on an Agilent Technologies/Oxford Diffraction Gemini CCD diffractometer (Agilent Technologies, Santa Clara, CA) .

\subsection{Electrochemical Measurements}

Homogeneous electrochemical data were collected using a Gamry Interface 1000E Potentiostat/Galvanostat/ZRA to perform all cyclic voltammetry (CV) experiments using 
glassy carbon working electrode (diameter $=6.5 \mathrm{~mm}$, surface area $\left.=0.07 \mathrm{~cm}^{2}\right), \mathrm{a}$ platinum wire counter electrode, and $\mathrm{Ag} / \mathrm{AgCl}$ reference electrode, with the exception of DCM which employed a silver wire as the reference electrode. All reported potentials are referenced versus ferrocenium/ferrocene $\left(\mathrm{Fc}^{+} / \mathrm{Fc}^{0}\right)$. The working electrode was polished with alumina slurry prior to use. Both the working and counter electrode were washed with water, ethanol, acetone and sonication in working solvent $(15 \mathrm{~min})$ prior to use. All CV experiments were performed under an inert atmosphere (either $\mathrm{N}_{2}$ or Ar) in a three-neck electrochemical cell that was washed and dried overnight prior to use.

For $\mathrm{CV}$ measurements a working solvent was selected and a $0.1 \mathrm{M}$ of tetrabutylammonium hexafluorophospate was added. This was then degassed for $15 \mathrm{~min}$ by sparging the working solvent with either argon or nitrogen. The solvent was scanned over the full window to ensure no contaminants were present. The compound of interest was then added and the system was then purged with argon or nitrogen for another 15 minutes to ensure no oxygen was present. The CVs of the complex could then be recorded. After completion of the CV experiment, a small quantity of ferrocene was added to act as an internal standard. For HER catalysis evaluation, glacial acetic acid was added, after which the solution was sparged with either nitrogen or argon to ensure a consistent concentration of acid. The system was allowed to rest after bubbling to ensure the reaction was diffusion controlled.

Heterogeneous evaluation of the activities of $\mathbf{2 , 2 8}$, and $\mathbf{2 9}$ for HER was carried out in a three-electrode glass electrochemical cell (RDE/RRDE cell without water jacket, Pine Research, Durham, NC) with $0.5 \mathrm{M} \mathrm{H}_{2} \mathrm{SO}_{4}$ (VWR, ACS grade) in twice-deionized millipore water (18.2 $\Omega \mathrm{cm}$ ). A graphite rod (Pine Research), in a protective fritted glass 
tube (Pine Research), was used as the counter electrode. $\mathrm{Ag} / \mathrm{AgCl}$ (CH Instruments, Bee Cave, TX) was used as the reference electrode. Measured potentials were calibrated vs. RHE after experiments were conducted (to prevent platinum contamination) by measuring the potential difference between a pristine platinum electrode (Standard Platinum Counter Electrode, Pine Research) and the reference electrode in $\mathrm{H}_{2}$-saturated $\mathrm{H}_{2} \mathrm{SO}_{4}$. High-purity $\mathrm{H}_{2}$ and $\mathrm{N}_{2}$ were used throughout these experiments (Welders Supply, Louisville, KY).

A Metrohm Autolab PGSTAT302N potentiostat/galvanostat, operating in potentiostat mode, was used to obtain polarization and frequency response analysis (FRA) data. Reductive cycling to activate the catalyst and evaluate its stability was carried out between $0 \mathrm{~V}$ and $-0.8 \mathrm{~V}$ vs. RHE at $50 \mathrm{mV} / \mathrm{sec}$. Linear sweep voltammetry was carried out intermittently throughout the cycling process, from $0 \mathrm{~V}$ to $-0.8 \mathrm{~V}$ vs. RHE at $2 \mathrm{mV} / \mathrm{sec}$, to evaluate the activity of the catalyst, at a relatively high resolution, after various amounts of usage at reductive potentials. FRA data was collected with an applied DC bias of $-0.3 \mathrm{~V}$ vs. RHE, starting at $100 \mathrm{kHz}$ and finishing at $0.02 \mathrm{~Hz}$, with $5 \mathrm{mV}_{\mathrm{RMS}}$ amplitude. The working electrode was rotated at 800 RPM throughout electrochemical characterization to assist the diffusion of $\mathrm{H}_{2}$ gas away from the catalyst surface. Measured values were iRcompensated by multiplying the measured current at each point by the real component of resistance measured at $100 \mathrm{kHz}$ and then subtracting these values from the corresponding applied potentials.

\subsection{Synthetic Procedures}

All reagents and solvents were purchased from commercial sources and used as received unless otherwise indicated. Reactions were conducted open to air and under ambient conditions unless otherwise noted. Solvents ethanol and methanol were grades 
absolute and $99.8 \%$, respectively. Xanthates ${ }^{79}$ hydrazinecarbothioic acid $O$-alkyl esters ${ }^{80}$, mono-keto-thiosemicarbazones, ${ }^{18,81} \mathrm{H}_{2} \mathrm{ATSM}, \mathrm{H}_{2} \mathrm{ATSDM}, \mathrm{H}_{2} \mathrm{ATSM}-\mathrm{F}_{6}, \mathbf{1}, \mathbf{2}$, and 3 were synthesized according to literature methods. ${ }^{8,32}$

$N, N^{\prime}$-bis(methylthiocarbamate)butane-2,3-diimine (4). The hydrazinecarbothioic acid $O$-methyl ester $(3.2 \mathrm{~g}, 30 \mathrm{mmol})$ was dissolved in ethanol $(50 \mathrm{~mL})$. To this solution 2,3butanedione $(1.3 \mathrm{~mL}, 15 \mathrm{mmol})$ was added by syringe with stirring. A catalytic amount (5 drops) of concentrated sulfuric acid was added resulting in a white precipitate. The suspension was stirred for $16 \mathrm{~h}$. The white precipitate was isolated by filtration and washed with ethanol and ether. Yield $=3.6 \mathrm{~g}(93 \%) .{ }^{1} \mathrm{H}$ NMR, $\left(\mathrm{C}_{2} \mathrm{D}_{6} \mathrm{SO}, 400 \mathrm{MHz}\right) \delta 2.11(\mathrm{~s}, 6 \mathrm{H})$ $4.03(\mathrm{~s}, 6 \mathrm{H}) 11.46(\mathrm{~s}, 1 \mathrm{H}) 11.77(\mathrm{~s}, 1 \mathrm{H}) .{ }^{13} \mathrm{C}\left\{{ }^{1} \mathrm{H}\right\} \mathrm{NMR},\left(\mathrm{CDCl}_{3}, 100 \mathrm{MHz}\right) \delta$ 12.3, 58.5, 117.8, 188.0. IR, $\mathrm{cm}^{-1}: 3240(\mathrm{w})$ and $1503(\mathrm{~m})(\mathrm{NH}) 1419(\mathrm{~m})(\mathrm{CH}) 1341(\mathrm{~m})$ and $1222(\mathrm{~s})$ (CN) 1137 (s) (CO) 1060 (s) (CS).

$N, N^{\prime}$-bis(ethylthiocarbamate)butane-2,3-diimine (5). Compound 5 was prepared using the same protocol as for 4 . Addition of 2,3-butanedione $(2.3 \mathrm{~mL}, 27 \mathrm{mmol})$ to hydrazinecarbothioic acid $O$-ethyl ester $(6.4 \mathrm{~g}, 53 \mathrm{mmol})$ in ethanol $(50 \mathrm{~mL})$ yielded 2 as a white solid. Yield $=7.74 \mathrm{~g}(98 \%) .{ }^{1} \mathrm{H} \mathrm{NMR},\left(\mathrm{C}_{3} \mathrm{D}_{7} \mathrm{NO}, 400 \mathrm{MHz}\right) \delta 1.51(\mathrm{br}, 6 \mathrm{H}) 2.46$ (br, 4H) 4.74 (s, 6H) 11.51 (s, 1H) 11.92 (s, 1H). IR, cm ${ }^{-1}: 3241$ (w) and $1498(\mathrm{~s})(\mathrm{NH})$ $1403(\mathrm{~m})(\mathrm{CH}) 1317(\mathrm{~m})$ and $1211(\mathrm{~s})(\mathrm{CN}) 1135$ (m) (CO) 1049 (m) (CS).

$N, N^{\prime}$-bis(n-propylthiocarbamate)butane-2,3-diimine (6). Compound 6 was prepared as described for 4 from hydrazinecarbothioic acid $O$-propyl ester $(2.1 \mathrm{~g}, 15 \mathrm{mmol})$ and 2,3butanedione $(0.66 \mathrm{~mL}, 7.5 \mathrm{mmol})$ in ethanol $(50 \mathrm{~mL})$. Yield $=0.95 \mathrm{~g}(40 \%) .{ }^{1} \mathrm{H}$ NMR, $\left(\mathrm{CDCl}_{3}, 400 \mathrm{MHz}\right) \delta 0.99(\mathrm{t}, 6 \mathrm{H}, J=7.28 \mathrm{~Hz}) 1.84$ (quint, 4H, $\left.J=6.24 \mathrm{~Hz}\right) 2.13(\mathrm{~s}, 6 \mathrm{H})$ $4.55(\mathrm{t}, 4 \mathrm{H}, J=6.22 \mathrm{~Hz}) 9.15(\mathrm{~s}, 2 \mathrm{H}) .{ }^{13} \mathrm{C}\left\{{ }^{1} \mathrm{H}\right\} \mathrm{NMR},\left(\mathrm{CDCl}_{3}, 100 \mathrm{MHz}\right) \delta 9.78,10.3$, 
21.9, 29.7, 187.6. IR, $\mathrm{cm}^{-1}: 3234(\mathrm{w})$ and 1497(s) (NH) $1402(\mathrm{~m})(\mathrm{CH}) 1334(\mathrm{~m})$ and 1207 (s) (CN) 1135 (s) (CO) 1052 (s) (CS).

$N, N^{\prime}$-bis(isopropylthiocarbamate)butane-2,3-diimine (7). Compound 7 was prepared as described for 4 from hydrazinecarbothioic acid $O$-isopropyl ester $(5.1 \mathrm{~g}, 38 \mathrm{mmol})$ and 2,3butanedione $(1.7 \mathrm{~mL}, 19 \mathrm{mmol})$ in ethanol $(50 \mathrm{~mL})$. Yield $=1.4 \mathrm{~g}(23 \%) .{ }^{1} \mathrm{H} \mathrm{NMR},\left(\mathrm{CDCl}_{3}\right.$, $400 \mathrm{MHz}) \delta 1.41(\mathrm{br}, 12 \mathrm{H}) 2.12(\mathrm{~s}, 6 \mathrm{H}) 5.63(\mathrm{br}, 2 \mathrm{H}) 9.10(\mathrm{~s}, 2 \mathrm{H}) .{ }^{13} \mathrm{C}\left\{{ }^{1} \mathrm{H}\right\} \mathrm{NMR},\left(\mathrm{CDCl}_{3}\right.$, $100 \mathrm{MHz}) \delta$ 10.3, 21.9. IR, $\mathrm{cm}^{-1}: 3234(\mathrm{w})$ and 1497(s) (NH) $1402(\mathrm{~m})(\mathrm{CH}) 1334(\mathrm{~m})$ and 1207 (s) (CN) 1135 (s) (CO) 1052 (s) (CS).

$N, N^{\prime}$-bis(octylthiocarbamate)butane-2,3-diimine (8). Compound 8 was prepared as described for 4 from hydrazinecarbothioic acid $O$-octyl ester $(2.72 \mathrm{~g}, 13 \mathrm{mmol})$ and 2,3butanedione $(0.58 \mathrm{~mL}, 6.7 \mathrm{mmol})$ in ethanol $\left(50 \mathrm{~mL}\right.$ ). . Yield $=1.77 \mathrm{~g}(58 \%) .{ }^{1} \mathrm{H}$ NMR, $\left(\mathrm{CDCl}_{3}, 400 \mathrm{MHz}\right) \delta 0.87(\mathrm{br}, 6 \mathrm{H}) 1.28(\mathrm{~m}, 16 \mathrm{H}) 1.43(\mathrm{br}, 4 \mathrm{H}) 1.81(\mathrm{br}, 4 \mathrm{H}) 2.13(\mathrm{~s}, 6 \mathrm{H})$ 4.59 (br, 4H) 9.12 (s, 2H). ${ }^{13} \mathrm{C}\left\{{ }^{1} \mathrm{H}\right\}$ NMR, $\left(\mathrm{CDCl}_{3}, 100 \mathrm{MHz}\right) \delta$ 14.1, 22.6, 25.8, 28.4, 29.1, 31.7. IR, $\mathrm{cm}^{-1}: 3230(\mathrm{w})$ and $1497(\mathrm{vs})(\mathrm{NH})$ 2950-2850 (m) and $1404(\mathrm{~s})(\mathrm{CH}) 1326(\mathrm{~s})$ and $1206(\mathrm{vs})(\mathrm{CN}) 1136(\mathrm{~s})(\mathrm{CO}) 1056(\mathrm{~s})(\mathrm{CS})$.

$N, N^{\prime}$-bis(ethylthiocarbamate)hexane-3,4-diimine (9). Compound 9 was prepared as described for 4 from hydrazinecarbothioic acid $O$-ethyl ester $(1.53 \mathrm{~g}, 13 \mathrm{mmol})$ and 3,4hexanedione $(0.77 \mathrm{~mL}, 6.5 \mathrm{mmol})$ in ethanol $(50 \mathrm{~mL})$. Yield $=1.2 \mathrm{~g}(60 \%) .{ }^{1} \mathrm{H}$ NMR, $\left(\mathrm{CDCl}_{3}, 400 \mathrm{MHz}\right) \delta 1.08(\mathrm{br}, 6 \mathrm{H}) 1.41(\mathrm{br}, 6 \mathrm{H}) 2.63(\mathrm{br}, 4 \mathrm{H}) 4.63(\mathrm{br}, 4 \mathrm{H}) 9.23(\mathrm{br}, 2 \mathrm{H})$. ${ }^{13} \mathrm{C}\left\{{ }^{1} \mathrm{H}\right\} \mathrm{NMR},\left(\mathrm{CDCl}_{3}, 100 \mathrm{MHz}\right) \delta 10.49,14.02,17.01,68.87,152.27,187.47 . \mathrm{IR}, \mathrm{cm}^{-1}$ : $3235(\mathrm{w})$ and $1498(\mathrm{~m})(\mathrm{NH}) 1433(\mathrm{w})(\mathrm{CH}) 1314(\mathrm{~m})$ and $1206(\mathrm{~s})(\mathrm{CN}) 1152(\mathrm{~m})(\mathrm{CO})$ 1059 (s) (CS). 
Cu(MTCB) (10). Compound 4 (0.273 g, $1.29 \mathrm{mmol})$ was suspended in methanol (25 mL, $100 \%)$. To this suspension copper(II) acetate monohydrate $(0.285 \mathrm{~g}, 1.49 \mathrm{mmol})$ was added with stirring giving a red-brown precipitate. The suspension was refluxed with stirring for $4 \mathrm{~h}$ and then allowed to cool to room temperature. The formed solid was filtered, washed with methanol then ether and air dried. Yield $=0.216 \mathrm{~g}(52 \%)$. X-ray quality crystals were obtained by slow evaporation of a DCM solution of $\mathbf{1 0}$ layered with ethanol. IR, $\mathrm{cm}^{-1}$ : 1471 (s) and 1398 (m) (CH) 1241 (vs) and 1218 (vs) (CN) 1091 (w) (CO) 948 (w) (CS) $848(\mathrm{w})(\mathrm{CuS}) 771(\mathrm{w})(\mathrm{CuN}) . \mathrm{UV}-$ Visible spectrum in DCM, nm, $\left(\mathrm{M}^{-1} \mathrm{~cm}^{-1}\right): 246$ $(16,000), 290(20,000), 486(2,680)$. EPR $g=2.061$. Elemental analysis calc. for $\mathrm{C}_{8} \mathrm{H}_{12} \mathrm{~S}_{2} \mathrm{O}_{2} \mathrm{~N}_{4} \mathrm{Cu}: \mathrm{C}, 29.67, \mathrm{H}, 3.73, \mathrm{~N}, 17.30, \mathrm{O}, 9.88, \mathrm{~S}, 19.80$. Found: C, 28.49, H, 3.58, $\mathrm{N}, 15.97, \mathrm{O}, 8.54, \mathrm{~S}, 17.87$. Mass spectrum calc. for $\mathrm{C}_{8} \mathrm{H}_{12} \mathrm{~S}_{2} \mathrm{O}_{2} \mathrm{~N}_{4} \mathrm{Cu}$ : 322.9698. Found: 322.9775.

$\mathbf{C u}(\mathbf{E T C B})$ (11). Compound 11 was prepared using the method described for 10 from 5 $(0.309 \mathrm{~g}, 1.29 \mathrm{mmol})$ and copper(II) acetate monohydrate $(0.285 \mathrm{~g}, 1.49 \mathrm{mmol})$ in methanol $(25 \mathrm{~mL})$. Complex 11 was isolated as a red-brown solid. Yield $=0.237 \mathrm{~g}(52 \%)$. X-ray quality crystals were obtained by using the technique described for 10. IR, $\mathrm{cm}^{-1}: 1413(\mathrm{~s})$ (CH) $1216(\mathrm{~s})(\mathrm{CN}) 1020$ (m) (CO) 867 (w) (CS) 792 (w) (CuS) 673 (w) (CuN). UVVisible spectrum in DCM, nm, $\left(\mathrm{M}^{-1} \mathrm{~cm}^{-1}\right): 248$ (16,300), 290 (22,700), 485 (2920). EPR $g_{\|}=2.131$ and $g_{\perp}=2.036$. Elemental analysis calc. for $\mathrm{C}_{10} \mathrm{H}_{16} \mathrm{~S}_{2} \mathrm{O}_{2} \mathrm{~N}_{4} \mathrm{Cu}: \mathrm{C}, 34.13, \mathrm{H}, 4.58$, $\mathrm{N}, 15.92, \mathrm{O}, 9.09, \mathrm{~S}, 18.22$. Found: C, 34.08, H, 4.40, N, 15.46, O, 9.18, S, 18.22. Mass spectrum calc. for $\mathrm{C}_{10} \mathrm{H}_{16} \mathrm{~S}_{2} \mathrm{O}_{2} \mathrm{~N}_{4} \mathrm{Cu}: 351.0011$. Found: 351.0086 .

$\mathrm{Cu}(\mathrm{PTCB})$ (12). Compound 12 was prepared using the method described for 10 from 6 $(0.408 \mathrm{~g}, 1.29 \mathrm{mmol})$ and copper(II) acetate monohydrate $(0.285 \mathrm{~g}, 1.49 \mathrm{mmol})$ in methanol 
$(25 \mathrm{~mL})$. Complex 12 was isolated as a red-brown solid. Yield $=0.322 \mathrm{~g}(68 \%)$. X-ray quality crystals were obtained by using the technique described for 10. IR, $\mathrm{cm}^{-1}: 1467$ (w) and $1423(\mathrm{~s})(\mathrm{CH}) 1236(\mathrm{~s})(\mathrm{CN}) 1093(\mathrm{w})(\mathrm{CO}) 956(\mathrm{~m})(\mathrm{CS}) 844$ (w) (CuS) $632(\mathrm{w})$ (CuN). UV-Visible spectrum in DCM, nm, $\left(\mathrm{M}^{-1} \mathrm{~cm}^{-1}\right): 248$ (16,600), $291(23,800), 484$ $(2,930)$. EPR $g_{\|}=2.127$ and $g_{\perp}=2.034$. Elemental analysis calc. for $\mathrm{C}_{12} \mathrm{H}_{20} \mathrm{~S}_{2} \mathrm{O}_{2} \mathrm{~N}_{4} \mathrm{Cu}: \mathrm{C}$, 37.93, H, 5.31, N, 14.74, O, 8.42, S, 16.88. Found: C, 37.99, H, 5.10, N, 14.67, O, 8.32, S, 16.75 Mass spectrum calc. for $\mathrm{C}_{12} \mathrm{H}_{20} \mathrm{~S}_{2} \mathrm{O}_{2} \mathrm{~N}_{4} \mathrm{Cu}$ : 379.0324. Found: 379.0417.

$\mathbf{C u}\left({ }^{i}\right.$ PTCB) (13). Compound 13 was prepared using the method described for 10 from 7 $(0.408 \mathrm{~g}, 1.29 \mathrm{mmol})$ and copper(II) acetate monohydrate $(0.285 \mathrm{~g}, 1.49 \mathrm{mmol})$ in methanol $(25 \mathrm{~mL})$. Complex 13 was isolated as a red-brown solid. Yield $=0.291 \mathrm{~g}(60 \%)$. X-ray quality crystals were obtained by using the technique described for 10. IR, $\mathrm{cm}^{-1}: 1427$ (s) (CH) 1213 (vs) (CN) 1103 (vs) (CO) 910 (m) (CS) 831 (w) (CuS) 671 (w) (CuN). Elemental analysis calc. for $\mathrm{C}_{12} \mathrm{H}_{20} \mathrm{~S}_{2} \mathrm{O}_{2} \mathrm{~N}_{4} \mathrm{Cu}$ : C, 37.93, H, 5.31, N, 14.74, O, 8.42, S, 16.88. Found: C, 37.38, H, 5.16, N, 14.39, O, 8.61, S, 17.10. UV-Visible spectrum in DCM, $\mathrm{nm},\left(\mathrm{M}^{-1} \mathrm{~cm}^{-1}\right): 248(16,500), 292(23,600), 484(3,210)$. EPR $g_{\|}=2.089$ and $g_{\perp}=2.042$. Mass spectrum calc. for $\mathrm{C}_{12} \mathrm{H}_{20} \mathrm{~S}_{2} \mathrm{O}_{2} \mathrm{~N}_{4} \mathrm{Cu}$ : 379.0324 . Found: 379.0401 .

$\mathbf{C u}(\mathbf{O T C B})$ (14). Compound 14 was prepared using the method described for 10 from 8 $(0.589 \mathrm{~g}, 1.29 \mathrm{mmol})$ and copper(II) acetate monohydrate $(0.285 \mathrm{~g}, 1.49 \mathrm{mmol})$ in methanol $(25 \mathrm{~mL})$. Complex 14 was isolated as a red-brown solid. Yield $=0.554 \mathrm{~g}(83 \%)$. X-ray quality crystals were obtained by using the technique described for $10 . \mathrm{IR}, \mathrm{cm}^{-1}: 2960-2852$ (m) and 1423 (s) (CH) 1238 (vs) (CN) 1079 (w) (CO) 946 (m) (CS) 846 (m) (CuS) 676 (w) (CuN). UV-Visible spectrum in DCM, nm, $\left(\mathrm{M}^{-1} \mathrm{~cm}^{-1}\right)$ : 248 (15,900), $291(23,600), 485$ (3,100). EPR $g_{\|}=2.130$ and $g_{\perp}=2.034$. Elemental analysis calc. for $\mathrm{C}_{22} \mathrm{H}_{40} \mathrm{~S}_{2} \mathrm{O}_{2} \mathrm{~N}_{4} \mathrm{Cu}$ : C, 
50.79, H, 7.75, N, 10.77, O, 6.15, S, 12.33. Found: C, 50.49, H, 7.57, N, 10.65, O, 6.34, S, 12.59. Mass spectrum calc. for $\mathrm{C}_{22} \mathrm{H}_{40} \mathrm{~S}_{2} \mathrm{O}_{2} \mathrm{~N}_{4} \mathrm{Cu}$ : 519.1889. Found: 519.1986.

Cu(ETCH) (15). Compound 15 was prepared using the method described for 10 from 9 $(0.408,1.29 \mathrm{mmol})$ and copper(II) acetate monohydrate $(0.285 \mathrm{~g}, 1.49 \mathrm{mmol})$ in methanol (25 mL). Complex 15 was isolated as a red-brown solid. Yield $=0.401 \mathrm{~g}(82 \%)$. X-ray quality crystals were obtained by using the technique described for $7 . \mathrm{IR}, \mathrm{cm}^{-1}: 1417$ (s) (CH) 1221 (s) (CN) 1016 (s) (CO) 974 (w) (CS) 874 (m) (CuS) 630 (m) (CuN). UV-Visible spectrum in DCM, nm, $\left(\mathrm{M}^{-1} \mathrm{~cm}^{-1}\right): 249(18,300), 292(25,000), 485(3,340)$. EPR $g_{\|}=2.124$ and $g_{\perp}=2.031$. Elemental analysis calc. for $\mathrm{C}_{12} \mathrm{H}_{20} \mathrm{~S}_{2} \mathrm{O}_{2} \mathrm{~N}_{4} \mathrm{Cu}: \mathrm{C}, 37.93, \mathrm{H}, 5.31, \mathrm{~N}, 14.74$, O, 8.42, S, 16.88. Found: C, 37.71, H, 4.96, N, 14.43, O, 8.33, S, 16.95. Mass spectrum calc. for $\mathrm{C}_{12} \mathrm{H}_{20} \mathrm{~S}_{2} \mathrm{O}_{2} \mathrm{~N}_{4} \mathrm{Cu}: 379.0324$. Found: 379.0315 .

H2MEB (16). Diacetyl-2-(4- $N$-methyl-3-thiosemicarbazone) (0.894 g, $6.3 \mathrm{mmol})$ was suspended in ethanol $(25 \mathrm{~mL})$, and hydrazinecarbothioic acid $O$-ethyl ester $(0.760 \mathrm{~g}, 6.3$ mmol) was added. A catalytic amount (6 drops) of concentrated sulfuric acid was added resulting in a white precipitate. The solid was isolated by filtration and washed with water then ethanol. Yield $=1.32 \mathrm{~g}(76 \%) .{ }^{1} \mathrm{H}$ NMR, $\left(\mathrm{C}_{2} \mathrm{D}_{6} \mathrm{SO}, 400 \mathrm{MHz}\right), \delta 1.28(\mathrm{br}, 3 \mathrm{H}), 2.11$ and $2.17(\mathrm{br}, 6 \mathrm{H}), 3.00(\mathrm{~s}, 3 \mathrm{H}), 8.35(\mathrm{~s}, 1 \mathrm{H}), 10.21(\mathrm{~s}, 1 \mathrm{H}) 11.31$ and $11.62(\mathrm{~s}, 1 \mathrm{H}) .{ }^{13} \mathrm{C}$ NMR, $\left(\mathrm{C}_{2} \mathrm{D}_{6} \mathrm{SO}, 100 \mathrm{MHz}\right) \delta 11.8,12.5,31.7,66.3,67.5,147.9,152.5,179.0,187.0 . \mathrm{IR}$, $\mathrm{cm}^{-1}: 3382,3253,1541,1494(\mathrm{NH}), 2991$ and $1411(\mathrm{CH}), 1304$ and $1217(\mathrm{CN}), 1130(\mathrm{CO})$, 1053 (CS). Elemental analysis calc. for $\mathrm{C}_{9} \mathrm{H}_{17} \mathrm{~S}_{2} \mathrm{ON}_{5}$ : C, 39.25, H, 6.22, N, 25.43. Found: C, 39.12, H, 6.16, N, 25.54.

$\mathrm{H}_{2}$ MPB (17). Compound 17 was prepared using the method for 16. Addition of hydrazinecarbothioic acid $O$-propyl ester $(0.1 .45 \mathrm{~g}, 11 \mathrm{mmol})$ to diacetyl-2-(4- $N$-methyl- 
3-thiosemicarbazone) $(1.58 \mathrm{~g}, 11 \mathrm{mmol})$ in ethanol $(25 \mathrm{~mL})$ yielding a white solid. Yield $=1.32 \mathrm{~g}(75 \%) .{ }^{1} \mathrm{H} \mathrm{NMR},\left(\mathrm{C}_{2} \mathrm{D}_{6} \mathrm{SO}, 400 \mathrm{MHz}\right), \delta 0.94$ (br, 3H) $1.70(\mathrm{br}, 2 \mathrm{H}), 2.11$ and $2.19(\mathrm{~s} / \mathrm{br}, 6 \mathrm{H}) 3.00(\mathrm{~s}, 3 \mathrm{H}) 4.41(\mathrm{br}, 2 \mathrm{H}) 8.43(\mathrm{~s}, 1 \mathrm{H}) 10.25(\mathrm{~s}, 1 \mathrm{H}) 11.35$ and $11.63(\mathrm{~s}$, 1H). ${ }^{13} \mathrm{C}$ NMR, $\left(\mathrm{C}_{2} \mathrm{D}_{6} \mathrm{SO}, 100 \mathrm{MHz}\right), \delta$ 10.8, 22.0, 31.7, 179.0. IR, $\mathrm{cm}^{-1}: 3382,3231,1540$, $1497(\mathrm{NH}), 2968$ and $1411(\mathrm{CH}), 1303$ and $1211(\mathrm{CN}), 1132(\mathrm{CO}), 1068(\mathrm{CS})$. Elemental analysis calc. for $\mathrm{C}_{10} \mathrm{H}_{19} \mathrm{~S}_{2} \mathrm{ON}_{5}$ : C, 41.50, H, 6.62, N, 24.20. Found: C, 41.31, H, 6.46, N, 24.02.

H2MEP (18). Compound 18 was prepared from using the method described for $\mathbf{1 6}$ Addition of hydrazinecarbothioic acid $O$-ethyl ester $(0.43 \mathrm{~g}, 3.6 \mathrm{mmol})$ to 1-phenyl-2propanone-1-(4- $N$-methyl-3-thiosemicarbazone) $(0.840 \mathrm{~g}, 3.6 \mathrm{mmol})$ in ethanol $(25 \mathrm{~mL})$ yielding a white solid. Yield $=0.871 \mathrm{~g}(72 \%) . \mathrm{IR}, \mathrm{cm}^{-1}: 3336,3223,1476,1542(\mathrm{NH})$, 2937 and $1417(\mathrm{CH}), 1301$ and $1219(\mathrm{CN}), 1195(\mathrm{CO}), 1081(\mathrm{CS})$. Elemental analysis calc. for $\mathrm{C}_{14} \mathrm{H}_{19} \mathrm{~S}_{2} \mathrm{ON}_{5}: \mathrm{C}, 49.83, \mathrm{H}, 5.67, \mathrm{~N}, 20.75$. Found: C, 49.14, H, 5.60, N, 20.18.

Cu(MEB) (19). Compound 16 (0.354 g, $1.29 \mathrm{mmol})$ was suspended in methanol (25 mL, $100 \%)$. To this suspension copper(II) acetate monohydrate $(0.285 \mathrm{~g}, 1.49 \mathrm{mmol})$ was added with stirring giving a red-brown precipitate. The suspension was refluxed with stirring for $4 \mathrm{~h}$ and then cooled to room temperature. The solid was then filtered and washed with methanol. Yield $=0.220 \mathrm{~g}(51 \%) . \mathrm{IR}, \mathrm{cm}^{-1}: 3305$ and $1561(\mathrm{NH}), 2937$ and $1439(\mathrm{CH})$, 1382 and $1217(\mathrm{CN}), 1132(\mathrm{CO}), 1025(\mathrm{CS}), 846(\mathrm{CuS}) 782(\mathrm{CuN})$. UV-Visible spectrum in DCM, nm, $\left(\mathrm{M}^{-1} \mathrm{~cm}^{-1}\right): 248(16,000), 309(20,000)$, shoulder around $346(11,000), 473$ $(5,100)$. EPR $g=2.061$. Elemental analysis calc. for $\mathrm{C}_{9} \mathrm{H}_{15} \mathrm{~S}_{2} \mathrm{ON}{ }_{5} \mathrm{Cu}: \mathrm{C}, 32.08, \mathrm{H}, 4.49$, $\mathrm{N}, 20.79$. Found: $\mathrm{C}, 31.53, \mathrm{H}, 4.34, \mathrm{~N}, 20.26$ Mass spectrum calc. for $\mathrm{C}_{9} \mathrm{H}_{15} \mathrm{~S}_{2} \mathrm{ON}_{5} \mathrm{Cu}$ : 336.00141. Found: 337.0080 . 
Cu(MPB) (20). Compound 20 was prepared using the same method as described for 19 from $17(0.373 \mathrm{~g}, 1.29 \mathrm{mmol})$ and copper(II) acetate monohydrate $(0.285 \mathrm{~g}, 1.49 \mathrm{mmol})$ in methanol $(25 \mathrm{~mL})$. Compound 20 was isolated as a red-brown solid. Yield $=0.200 \mathrm{~g}(44 \%)$. IR, $\mathrm{cm}^{-1}$ : 3329 and $1562(\mathrm{NH}), 2972$ and $1465(\mathrm{CH}), 1382$ and $1219(\mathrm{CN}), 1019(\mathrm{CO}), 974$ (CS), $872(\mathrm{CuS}), 758(\mathrm{CuN})$. UV-Visible spectrum in DCM, nm, $\left(\mathrm{M}^{-1} \mathrm{~cm}^{-1}\right): 249(13,000)$, $310(17,000)$, shoulder around $346(8,700), 477(4,300)$. EPR $g_{\|}=2.116$ and $g_{\perp}=2.030$. Elemental analysis calc. for $\mathrm{C}_{10} \mathrm{H}_{17} \mathrm{~S}_{2} \mathrm{ON}_{5} \mathrm{Cu}$ : C, 34.22, H, 4.88, N, 19.96. Found: C, 34.17, $\mathrm{H}, 4.83, \mathrm{~N}, 20.01$. Mass spectrum calc. for $\mathrm{C}_{10} \mathrm{H}_{17} \mathrm{~S}_{2} \mathrm{ON} \mathrm{N}_{5} \mathrm{Cu}: 350.01706$. Found: 351.0239 .

Cu(MEP) (21). Compound 21 was prepared using the same method as described for 19 from $18(0.435 \mathrm{~g}, 1.29 \mathrm{mmol})$ and copper(II) acetate monohydrate $(0.285 \mathrm{~g}, 1.49 \mathrm{mmol})$ in methanol $(25 \mathrm{~mL})$. Compound 21 was isolated as a red-brown solid. Yield $=0.146 \mathrm{~g}(29 \%)$. IR cm ${ }^{-1} .3324$ and $1538(\mathrm{NH}), 2973$ and $1428(\mathrm{CH}), 1382$ and $1219(\mathrm{CN}), 1019(\mathrm{CO}), 974$ (CS), $872(\mathrm{CuS}) 758(\mathrm{CuN})$. UV-Visible spectrum in DCM, nm, $\left(\mathrm{M}^{-1} \mathrm{~cm}^{-1}\right)$ : Shoulder around $263(16,000), 309(20,000)$, shoulder around $357(13,000), 485(5,500)$. EPR $g_{\|}=$ 2.135 and $g_{\perp}=2.032$. Elemental analysis calc. for $\mathrm{C}_{14} \mathrm{H}_{17} \mathrm{~S}_{2} \mathrm{ON}{ }_{5} \mathrm{Cu}: \mathrm{C}, 42.14, \mathrm{H}, 4.29, \mathrm{~N}$, 17.55. Found: $\mathrm{C}, 41.84, \mathrm{H}, 4.22, \mathrm{~N}, 17.53$. Mass spectrum calc. for $\mathrm{C}_{14} \mathrm{H}_{17} \mathrm{~S}_{2} \mathrm{ON}{ }_{5} \mathrm{Cu}$ : 398.01706. Found: 339.0238 .

Ni(MEB) (22). Compound 16 (0.354 g, $1.29 \mathrm{mmol})$ was suspended in methanol (25 mL). To this suspension nickel(II) acetate tetrahydrate $(0.353 \mathrm{~g}, 1.49 \mathrm{mmol})$ was added with stirring giving a dark brown precipitate. The suspension was refluxed for $4 \mathrm{~h}$ and then cooled to room temperature prior to being placed in a freezer overnight. The formed solid was filtered cold then washed with methanol. Yield $=0.326 \mathrm{~g}(76 \%) .{ }^{1} \mathrm{H}$ NMR, $\left(\mathrm{C}_{2} \mathrm{D}_{6} \mathrm{SO}\right.$, $400 \mathrm{MHz}), \delta 1.21(\mathrm{t}, 3 \mathrm{H}) 2.00(\mathrm{~s}, 6 \mathrm{H}) 2.81(\mathrm{~s}, 3 \mathrm{H}) 4.20(\mathrm{br}, 2 \mathrm{H}) 8.03(\mathrm{~s}, 1 \mathrm{H}) . \mathrm{IR}, \mathrm{cm}^{-1}: 3414$ 
and $1553(\mathrm{NH}), 2937$ and $1402(\mathrm{CH}), 1382$ and $1216(\mathrm{CN}), 1080(\mathrm{CO}), 1014(\mathrm{CS}), 872$ (NiS), $782(\mathrm{NiN})$. UV-Visible spectrum in DCM, nm, $\left(\mathrm{M}^{-1} \mathrm{~cm}^{-1}\right): 255(38,000)$, shoulder around $326(6,400), 389$ (12,000). Elemental analysis calc. for $\mathrm{C}_{9} \mathrm{H}_{15} \mathrm{~S}_{2} \mathrm{ON} \mathrm{N}_{5} \mathrm{Ni}$ : C, 32.55, $\mathrm{H}, 4.55, \mathrm{~N}, 21.09$. Found: $\mathrm{C}, 32.09, \mathrm{H}, 4.44, \mathrm{~N}, 20.54$. Mass spectrum calc. for $\mathrm{C}_{9} \mathrm{H}_{15} \mathrm{~S}_{2} \mathrm{ON}_{5} \mathrm{Ni}: 331.00716$. Found: 331.0062 and 332.0140.

Ni(MPB) (23). Compound 23 was prepared using the method described for 22 from 17 $(0.373 \mathrm{~g}, 1.29 \mathrm{mmol})$ and nickel(II) acetate tetrahydrate $(0.353 \mathrm{~g}, 1.49 \mathrm{mmol})$ in methanol (25 mL). Compound 23 was isolated as a dark brown solid. Yield $=0.250 \mathrm{~g}(56 \%) .{ }^{1} \mathrm{H}$ NMR, $\left(\mathrm{C}_{2} \mathrm{D}_{6} \mathrm{SO}, 400 \mathrm{MHz}\right) \delta 0.87(\mathrm{t}, 3 \mathrm{H}) 1.59(\mathrm{br}, 2 \mathrm{H}) 2.01(\mathrm{br}, 6 \mathrm{H}) 2.81(\mathrm{~s}, 3 \mathrm{H}) 4.12(\mathrm{br}$, 2H) $8.02(\mathrm{~s}, 1 \mathrm{H}) . \mathrm{IR}, \mathrm{cm}^{-1}: 3341$ and $1562(\mathrm{NH}), 29333$ and $1493(\mathrm{CH}), 1307$ and 1225 (CN), 1084 (CO), 1042 (CS), 855 (NiS) 735 (NiN). UV-Visible spectrum in DCM, nm, $\left(\mathrm{M}^{-1} \mathrm{~cm}^{-1}\right): 255(32,000)$, shoulder around $330(4,500), 388(11,000)$. Elemental analysis calc. for $\mathrm{C}_{10} \mathrm{H}_{17} \mathrm{~S}_{2} \mathrm{ON} \mathrm{N}_{5} \mathrm{Ni}$ : C, 34.70, H, 4.95, N, 20.24. Found: C, 34.49, H, 4.86, N, 19.92 . Mass spectrum calc. for $\mathrm{C}_{10} \mathrm{H}_{17} \mathrm{~S}_{2} \mathrm{ON}_{5} \mathrm{Ni}$ : 345.02281 . Found: 345.0128 and 346.0296.

Ni(MEP) (24). Compound 24 was prepared using the method described for 22 from 18 $(0.435 \mathrm{~g}, 1.29 \mathrm{mmol})$ and nickel(II) acetate tetrahydrate $(0.353 \mathrm{~g}, 1.49 \mathrm{mmol})$ in methanol (25 mL). Compound 24 was isolated as a dark brown solid. Yield $=0.332 \mathrm{~g}(65 \%) .{ }^{1} \mathrm{H}$ NMR, $\left(\mathrm{C}_{2} \mathrm{D}_{6} \mathrm{SO}, 400 \mathrm{MHz}\right) \delta 1.12(\mathrm{t}, 3 \mathrm{H}) 1.80(\mathrm{~s}, 3 \mathrm{H}) 2.85(\mathrm{~s}, 3 \mathrm{H}) 7.46(\mathrm{br}, 5 \mathrm{H}) 8.19(\mathrm{~s}$, 1H). IR, $\mathrm{cm}^{-1}:$ 3408, 3256, and $1544(\mathrm{NH}), 2975$ and $1500(\mathrm{CH}), 1332$ and $1226(\mathrm{CN})$, 1016 (CO), 975 (CS), 874 (NiS) 758 (NiN). UV-Visible spectrum in DCM, nm, $\left(\mathrm{M}^{-1} \mathrm{~cm}^{-}\right.$ $\left.{ }^{1}\right)$ : $252(30,000), 342(7,600), 405(11,000)$. Elemental analysis calc. for $\mathrm{C}_{14} \mathrm{H}_{17} \mathrm{~S}_{2} \mathrm{ON}_{5} \mathrm{Ni}$ : C, 42.66, H, 4.35, N, 17.77. Found: C, 42.85, H, 4.46, N, 17.57. Mass spectrum calc. for $\mathrm{C}_{14} \mathrm{H}_{17} \mathrm{~S}_{2} \mathrm{ON}_{5} \mathrm{Ni}: 393.02281$. Found: 393.0214 and 394.0295. 
Zn(MEB) (25). Compound 16 (0.354 g, $1.29 \mathrm{mmol})$ was suspended in methanol (25 mL). To this suspension zinc(II) acetate dihydrate $(0.307 \mathrm{~g}, 1.49 \mathrm{mmol})$ was added with stirring giving a yellow-orange precipitate. The suspension was refluxed for $4 \mathrm{~h}$ and then cooled to room temperature. The formed solid was filtered and then washed with methanol. Yield = $0.275 \mathrm{~g}(63 \%) .{ }^{1} \mathrm{H} \mathrm{NMR},\left(\mathrm{C}_{2} \mathrm{D}_{6} \mathrm{SO}, 400 \mathrm{MHz}\right), \delta 1.26(\mathrm{t}, 3 \mathrm{H}) 2.22(\mathrm{~s}, 3 \mathrm{H}) 2.25(\mathrm{~s}, 3 \mathrm{H}) 2.84$ (3H) 4.30 (br, 2H) $7.48(1 \mathrm{H}) .{ }^{13} \mathrm{C} \mathrm{NMR,}\left(\mathrm{C}_{2} \mathrm{D}_{6} \mathrm{SO}, 100 \mathrm{MHz}\right) \delta 14.2,14.9,64.0,110.0 . \mathrm{IR}$, $\mathrm{cm}^{-1}: 3337$ and $1557(\mathrm{NH}), 2930$ and $1494(\mathrm{CH}), 1397$ and $1227(\mathrm{CN}), 1082(\mathrm{CO}), 1027$ (CS), $841(\mathrm{ZnS}), 774(\mathrm{ZnN}) . \mathrm{UV}-$ Visible spectrum in DCM, nm, $\left(\mathrm{M}^{-1} \mathrm{~cm}^{-1}\right): 243(12,000)$, 299 (9,900), 422 (8,700). Elemental analysis calc. for $\mathrm{C}_{9} \mathrm{H}_{15} \mathrm{~S}_{2} \mathrm{ON}_{5} \mathrm{Zn}$ : C, 31.91, H, 4.46, N, 20.67. Found: C, 31.92, H, 4.45, N, 20.67. Mass spectrum calc. for $\mathrm{C}_{9} \mathrm{H}_{15} \mathrm{~S}_{2} \mathrm{ON}_{5} \mathrm{Zn}: 337.00096$. Found: 338.2153.

Zn(MPB) (26). Compound 26 was prepared using the same method as described for 25 from $17(0.373 \mathrm{~g}, 1.29 \mathrm{mmol})$ and zinc(II) acetate dihydrate $(0.307 \mathrm{~g}, 1.49 \mathrm{mmol})$ in methanol $(25 \mathrm{~mL})$. Compound $\mathbf{2 6}$ was isolated as a yellow-orange precipitate. Yield = $0.424 \mathrm{~g}(93 \%) .{ }^{1} \mathrm{H}$ NMR, $\left(\mathrm{C}_{2} \mathrm{D}_{6} \mathrm{SO}, 400 \mathrm{MHz}\right), \delta 0.92(\mathrm{t}, 3 \mathrm{H}) 1.66(\mathrm{br}, 2 \mathrm{H}) 2.20(\mathrm{~s}, 3 \mathrm{H})$ $2.22(\mathrm{~s}, 3 \mathrm{H}) 2.85(\mathrm{~s}, 3 \mathrm{H}) 4.21(\mathrm{br}, 2 \mathrm{H}) 7.48(\mathrm{~s}, 1 \mathrm{H}) .{ }^{13} \mathrm{C} \mathrm{NMR},\left(\mathrm{C}_{2} \mathrm{D}_{6} \mathrm{SO}, 100 \mathrm{MHz}\right), \delta 10.9$, 14.9, 22.3, 69.7, 110.0. IR, $\mathrm{cm}^{-1}: 3367,3311$, and $1549(\mathrm{NH}), 2973$ and $1491(\mathrm{CH}), 1372$ and $1209(\mathrm{CN}), 1122(\mathrm{CO}), 1079(\mathrm{CS}), 836(\mathrm{ZnS}), 783(\mathrm{ZnN})$. UV-Visible spectrum in DCM, nm, $\left(\mathrm{M}^{-1} \mathrm{~cm}^{-1}\right): 243$ (10,000), 299 (7,400), 423 (7,500). Elemental analysis calc. for $\mathrm{C}_{10} \mathrm{H}_{17} \mathrm{~S}_{2} \mathrm{ON}_{5} \mathrm{Zn} \cdot \mathrm{CH}_{3} \mathrm{OH}: \mathrm{C}, 34.33, \mathrm{H}, 5.50, \mathrm{~N}, 18.20$. Found: C, 33.99, H, 5.25, N, 18.12. Mass spectrum calc. for $\mathrm{C}_{10} \mathrm{H}_{17} \mathrm{~S}_{2} \mathrm{ON}_{5} \mathrm{Zn}: 351.0166$. Found: 352.07 .

Zn(MEP) (27). Compound 27 was prepared using the same method as described for 25 from $18(0.435 \mathrm{~g}, 1.29 \mathrm{mmol})$ and zinc(II) acetate dihydrate $(0.307 \mathrm{~g}, 1.49 \mathrm{mmol})$ in 
methanol $(25 \mathrm{~mL})$. Compound 27 was isolated as a yellow-orange precipitate. Yield $=$ $0.322 \mathrm{~g}(62 \%) .{ }^{1} \mathrm{H}$ NMR, $\left(\mathrm{C}_{2} \mathrm{D}_{6} \mathrm{SO}, 400 \mathrm{MHz}\right), \delta 1.14(\mathrm{t}, 3 \mathrm{H}) 2.03(\mathrm{~s}, 3 \mathrm{H}) 2.87(\mathrm{~s}, 3 \mathrm{H}) 4.08$ (br, 2H) 7.45 (br, 5H) 7.61 (s, 1H). ${ }^{13} \mathrm{C}$ NMR, $\left(\mathrm{C}_{2} \mathrm{D}_{6} \mathrm{SO}, 100 \mathrm{MHz}\right), \delta$ 14.8, 16.2, 110.0, 128.4, 129.5, 129.7, 132.9. IR, $\mathrm{cm}^{-1}: 3351$ and $1541(\mathrm{NH}), 2971$ and $1481(\mathrm{CH}), 1392$ and $1221(\mathrm{CN}), 1019$ (CO), $970(\mathrm{CS}), 801(\mathrm{ZnS}), 756$ (ZnN). UV-Visible spectrum in DCM, $\mathrm{nm},\left(\mathrm{M}^{-1} \mathrm{~cm}^{-1}\right): 269(11,000), 326(8,100), 430(7,300)$. Elemental analysis calc. for $\mathrm{C}_{14} \mathrm{H}_{17} \mathrm{~S}_{2} \mathrm{ON} \mathrm{N}_{5} \mathrm{Zn}: \mathrm{C}, 41.95, \mathrm{H}, 4.27, \mathrm{~N}, 17.47$. Found: C, 40.65, H, 4.08, N, 19.97. Mass spectrum calc. for $\mathrm{C}_{14} \mathrm{H}_{17} \mathrm{~S}_{2} \mathrm{ON}_{5} \mathrm{Zn}: 399.01661$. Found: 400.3753 .

Ni(ATSDM) (28). The ligand $\mathrm{H}_{2} \mathrm{ATSDM}(0.28 \mathrm{~g}, 0.96 \mathrm{mmol})$ was suspended in methanol $(25 \mathrm{~mL})$. To this suspension, nickel (II) acetate tetrahydrate $(0.25 \mathrm{~g}, 1.0 \mathrm{mmol})$ was added with stirring giving a dark green-brown precipitate. The suspension was refluxed for $4 \mathrm{~h}$ and then cooled to room temperature prior to being placed in a freezer overnight. The formed solid was filtered cold and washed with water then ethanol giving a red-brown solid. Yield $=0.293 \mathrm{~g}(88 \%)$. X-ray quality crystals were obtained by dissolving $\mathrm{Ni}(\mathrm{ATSDM})$ in acetonitrile, which was then layered with ether and allowed to slowly evaporate. ${ }^{1} \mathrm{H}$ NMR, $\left(\mathrm{C}_{2} \mathrm{D}_{6} \mathrm{SO}, 400 \mathrm{MHz}\right) \delta 1.87$ (s, 3H) 3.10 (s, 6H). IR, $\mathrm{cm}^{-1}: 2910(\mathrm{w})$ and $1501(\mathrm{~s})(\mathrm{CH}) 1366(\mathrm{vs})$ and $1082(\mathrm{~m})(\mathrm{C}-\mathrm{N}) 1322(\mathrm{vs})$ and $1258(\mathrm{~s})(\mathrm{C}=\mathrm{N}) 1006(\mathrm{w})$ (CS) $900(\mathrm{~m})(\mathrm{NiS}) 783(\mathrm{w})(\mathrm{NiN})$. UV-Visible spectrum in acetonitrile, $\mathrm{nm},\left(\mathrm{M}^{-1} \mathrm{~cm}^{-1}\right)$ : 256(23,000), 411 (11,000), $436(11,000)$. Elemental analysis calc. for $\mathrm{C}_{10} \mathrm{H}_{18} \mathrm{~N}_{6} \mathrm{~S}_{2} \mathrm{Ni}$ : C, 34.80, H, 5.26, N, 24.35. Found: C, 34.57, H, 5.20, N, 24.12. Mass spectrum calc. for $\mathrm{C}_{10} \mathrm{H}_{18} \mathrm{~N}_{6} \mathrm{~S}_{2} \mathrm{Ni}: 344.03878$ Found: 343.80 and 344.80 .

Ni(ATSM-F6) (29). The ligand $\mathrm{H}_{2} \mathrm{ATSM} \mathrm{F}_{6}(0.19 \mathrm{~g}, 0.48 \mathrm{mmol})$ was suspended in methanol (20 mL). To this suspension, nickel(II) acetate tetrahydrate $(0.15 \mathrm{~g}, 0.60 \mathrm{mmol})$ 
was added with stirring, giving a red-brown precipitate. The suspension was refluxed for 4 $\mathrm{h}$ and then cooled to room temperature prior to being placed in a freezer overnight. The formed solid was filtered cold and washed with water then ethanol giving a red-brown solid. Yield $=0.087 \mathrm{~g}(40 \%)$. X-ray quality crystals were obtained by dissolving $\operatorname{Ni}\left(\right.$ ATSM $\left._{-} \mathrm{F}_{6}\right)$ in acetonitrile, which was then layered with ether and allowed to slowly

evaporate. ${ }^{1} \mathrm{H}$ NMR, $\left(\mathrm{C}_{2} \mathrm{D}_{6} \mathrm{SO}, 400 \mathrm{MHz}\right), \delta 1.94$ (s, 3H) 4.00 (br, 2H) $8.29(\mathrm{~s}, 1 \mathrm{H}) .{ }^{13} \mathrm{C}$ NMR, $\left(\mathrm{C}_{2} \mathrm{D}_{6} \mathrm{SO}, 100 \mathrm{MHz}\right), \delta 178.1,158.8,124.9(\mathrm{q}, J=280 \mathrm{~Hz}), 46.3(\mathrm{q}, J=33 \mathrm{~Hz}) 14.4$. IR cm ${ }^{-1}: 3462(\mathrm{~m})(\mathrm{NH}) 1514(\mathrm{~s})$ and $1476(\mathrm{~s})(\mathrm{CH}) 1271(\mathrm{~s})$ and $1218(\mathrm{~m})(\mathrm{CN}) 1138(\mathrm{vs})$ (CF) $941(\mathrm{w})(\mathrm{NiS}) 832(\mathrm{w})(\mathrm{NiN})$. UV-Visible spectrum in acetonitrile, $\mathrm{nm},\left(\mathrm{M}^{-1} \mathrm{~cm}^{-1}\right)$ : 259 (24,000), $328(8,200), 392$ (9,100). Elemental analysis calc. for $\mathrm{C}_{10} \mathrm{H}_{12} \mathrm{~N}_{6} \mathrm{~S}_{2} \mathrm{~F}_{6} \mathrm{Ni}$ : C, 26.51, H, 2.67, N, 18.55, F, 25.16. Found: C, 26.36, H, 2.59, N, 18.29, F, 25.10. Mass spectrum calc. for $\mathrm{C}_{10} \mathrm{H}_{18} \mathrm{~N}_{6} \mathrm{~S}_{2} \mathrm{Ni}$ : 451.98223 Found: 452.87 .

\subsection{Crystallographic Studies}

Single crystal X-ray diffraction studies were conducted on complexes 10-15, 18, 19, 21, 22, 28, and 29. The CCDC deposit numbers and references can be found in Table 2-1. Suitable crystals for X-ray diffraction of $\mathbf{1 0 - 1 5}, \mathbf{1 8}, \mathbf{1 9}, \mathbf{2 1}$, and 22 were grown from a sealed tube in which dichloromethane was layered with ethanol. For $\mathbf{2 8}$ and 29, crystals were grown from a sealed tube in which acetonitrile was layered with diethyl ether.

\begin{tabular}{lll}
\multicolumn{3}{l}{ Table 2-1. CCDC and publication reference information } \\
\hline Compound & CCDC number & Reference \\
\hline $\mathbf{1 0}$ & 1517907 & 82 \\
$\mathbf{1 1}$ & 1517908 & 82 \\
$\mathbf{1 2}$ & 1517909 & 82 \\
$\mathbf{1 3}$ & 1517910 & 82 \\
$\mathbf{1 4}$ & 1517911 & 82 \\
$\mathbf{1 5}$ & 1517912 & 82 \\
$\mathbf{1 8}$ & N/A & 83 \\
$\mathbf{1 9}$ & N/A & 83 \\
$\mathbf{2 1}$ & N/A & 83 \\
$\mathbf{2 2}$ & N/A & 83 \\
$\mathbf{2 8}$ & 1866543 & 84 \\
$\mathbf{2 9}$ & 1866544 & 84
\end{tabular}


Crystals 10, 11, and 29 were mounted on a CryoLoop and $12-15$ and 28 were mounted on a glass fiber for collection of X-ray data on an Agilent Technologies/Oxford Diffraction Gemini CCD diffractometer. The CrysAlisPro ${ }^{85}$ CCD software package (v 1.171.36.32) was used to acquire $\omega$-scan exposures of data at $100 \mathrm{~K}$ using monochromated MoK $\alpha$ radiation $(0.71073 \AA)$ from a sealed tube. Frame data were processed using CrysAlisPro ${ }^{85}$ RED to determine final unit cell parameters to produce raw hkl data that were then corrected for absorption using SCALE3 ABSPACK. ${ }^{86}$ The structures of 10-15 were solved by Direct Methods using SHELX ${ }^{87}$ and refined by least squares methods on $\mathrm{F}^{2}$ using SHELXL-97. ${ }^{87}$ Crystals 28 and 29 were solved by Direct Methods using SHELXS ${ }^{87}$ and refined by least squares methods on $\mathrm{F}^{2}$ using SHELXL ${ }^{87}$ For all crystals non-hydrogen atoms were refined anisotropically.

For crystals 11-15 and 28, all hydrogens atoms were located by difference maps and refined isotropically. For $\mathbf{1 0}$ and $\mathbf{2 9}$ methyl hydrogen atoms were placed in their geometrically generated positions and refined as a riding model and these atoms were assigned $\mathrm{U}(\mathrm{H})=1.5 \mathrm{x}$ Ueq. The imine $\mathrm{NH}$ hydrogen atoms of $\mathbf{2 9}$ were located by difference maps and refined isotropically. For all unique reflections the final anisotropic full matrix least-squares refinement on $\mathrm{F}^{2}$ for all variables converged with R1, wR2, GOF as listed in the Tables 2-2 to 2-4. 
Table 2-2. Crystal data and structural refinement for 10-13

\begin{tabular}{|c|c|c|c|c|}
\hline Compound & 10 & 11 & 12 & 13 \\
\hline Empirical formula & $\mathrm{C}_{8} \mathrm{H}_{12} \mathrm{CuN}_{4} \mathrm{O}_{2} \mathrm{~S}_{2}$ & $\mathrm{C}_{10} \mathrm{H}_{16} \mathrm{CuN}_{4} \mathrm{O}_{2} \mathrm{~S}_{2}$ & $\mathrm{C}_{12} \mathrm{H}_{20} \mathrm{CuN}_{4} \mathrm{O}_{2} \mathrm{~S}_{2}$ & $\mathrm{C}_{12} \mathrm{H}_{20} \mathrm{CuN}_{4} \mathrm{O}_{2} \mathrm{~S}_{2}$ \\
\hline Formula weight & 323.89 & 351.93 & 379.98 & 379.98 \\
\hline Temperature (K) & $99.95(10)$ & $100.05(10)$ & $99.95(10)$ & $99.95(10)$ \\
\hline Wavelength $(\AA)$ & 0.71073 & 0.71073 & 0.71073 & 0.71073 \\
\hline Crystal system & Orthorhombic & Triclinic & Orthorhombic & Monoclinic \\
\hline Space group & Pbcn & $P-1$ & Pbcn & $P 2{ }_{1} / c$ \\
\hline Unit cell dimension & $16.8510(16)$ & $8.5267(4)$ & 22.6633(9) & $14.6103(8)$ \\
\hline $\mathrm{a}(\AA)$ & $8.8146(7)$ & $8.7430(3)$ & $9.0529(3)$ & $16.3511(6)$ \\
\hline $\mathrm{b}(\AA)$ & $8.2090(8)$ & $10.4065(4)$ & $7.8296(2)$ & $7.0344(8)$ \\
\hline c $(\AA)$ & 90 & $80.502(3)$ & 90 & 90 \\
\hline$\alpha\left(^{\circ}\right)$ & 90 & $72.838(4)$ & 90 & $98.413(5)$ \\
\hline$\beta\left(^{\circ}\right)$ & 90 & $70.532(4)$ & 90 & 90 \\
\hline \multicolumn{5}{|l|}{$\gamma\left(\left(^{\circ}\right)\right.$} \\
\hline Volume $\left(\AA^{3}\right)$ & $1219.3(2)$ & $696.95(5)$ & $1606.38(10)$ & $1662.40(14)$ \\
\hline $\mathrm{Z}$ & 4 & 2 & 4 & 4 \\
\hline$\rho_{\text {calcd. }}\left(\mathrm{Mg} / \mathrm{m}^{3}\right)$ & 1.764 & 1.677 & 1.571 & 1.518 \\
\hline Abs. coeff. $\left(\mathrm{mm}^{-1}\right)$ & 2.128 & 1.869 & 1.628 & 1.573 \\
\hline $\mathrm{F}(000)$ & 660 & 362 & 788 & 788 \\
\hline Crystal colour, habit & Thin orange plate & Dark purple plate & Orange plate & Orange plate \\
\hline Crystal size $\left(\mathrm{mm}^{3}\right)$ & $0.18 \times 0.18 \times 0.03$ & $0.17 \times 0.08 \times 0.03$ & $0.28 \times 0.07 \times 0.03$ & $0.33 \times 0.10 \times 0.02$ \\
\hline$\theta$ range data collection $\left(^{\circ}\right)$ & 3.60 to 25.35 & 3.33 to $27.14^{\circ}$ & 3.51 to $27.09^{\circ}$ & 3.30 to $26.10^{\circ}$ \\
\hline \multirow[t]{3}{*}{ Index ranges } & $-20 \leq h \leq 19$ & $-10 \leq h \leq 10$ & $-29 \leq \mathrm{h} \leq 26$ & $-18 \leq \mathrm{h} \leq 18$ \\
\hline & $-10 \leq \mathrm{k} \leq 9$ & $-11 \leq \mathrm{k} \leq 11$ & $-11 \leq \mathrm{k} \leq 10$ & $-20 \leq \mathrm{k} \leq 20$ \\
\hline & $-9 \leq 1 \leq 9$ & $-13 \leq 1 \leq 13$ & $-10 \leq 1 \leq 10$ & $-8 \leq 1 \leq 8$ \\
\hline Reflections collected & 6029 & $151 \overline{6} 1$ & 9630 & 13215 \\
\hline \multirow[t]{2}{*}{ Independent reflections } & 1119 & 3074 & 1772 & 3292 \\
\hline & {$[\mathrm{R}(\mathrm{int})=0.0503]$} & {$[\mathrm{R}(\mathrm{int})=0.0257]$} & {$[\mathrm{R}(\mathrm{int})=0.0337]$} & {$[\mathrm{R}($ int $)=0.050]$} \\
\hline Completeness to $\theta$ max & $99.8 \%$ & $99.8 \%$ & $99.8 \%$ & $99.5 \%$ \\
\hline Absorption correction & Multi-scan & Multi-scan & Multi-scan & Multi-scan \\
\hline Max. and min. trans. & 1.000 and 0.517 & 1.000 and 0.865 & 1.000 and 0.864 & 1.000 and 0.656 \\
\hline Refinement method & $\begin{array}{l}\text { Full-matrix least- } \\
\text { squares on } \mathrm{F}^{2}\end{array}$ & $\begin{array}{l}\text { Full-matrix least- } \\
\text { squares on } \mathrm{F}^{2}\end{array}$ & $\begin{array}{l}\text { Full-matrix least- } \\
\text { squares on } \mathrm{F}^{2}\end{array}$ & $\begin{array}{l}\text { Full-matrix least- } \\
\text { squares on } \mathrm{F}^{2}\end{array}$ \\
\hline Data/restraints/params & $1119 / 0 / 80$ & $3074 / 0 / 236$ & $1772 / 0 / 136$ & $3292 / 0 / 270$ \\
\hline Goodness-of-fit on $\mathrm{F}^{2}$ & 1.072 & 1.049 & 1.034 & 1.043 \\
\hline Final $\quad \mathrm{R} \quad$ indices & $\mathrm{R} 1=0.0523$ & $\mathrm{R} 1=0.0206$ & $\mathrm{R} 1=0.0259$ & $\mathrm{R} 1=0.039$ \\
\hline$[\mathrm{I}>2 \sigma(\mathrm{I})]^{\mathrm{a}, \mathrm{b}}$ & $\mathrm{wR} 2=0.0723$ & $\mathrm{wR} 2=0.0519$ & $\mathrm{wR} 2=0.0637$ & $w R 2=0.079$ \\
\hline $\mathrm{R}$ indices (all data) & $\mathrm{R} 1=0.0867$ & $\mathrm{R} 1=0.0229$ & $\mathrm{R} 1=0.0327$ & $\mathrm{R} 1=0.054$ \\
\hline & $\mathrm{wR} 2=0.0792$ & $\mathrm{wR} 2=0.0532$ & $w R 2=0.0672$ & $w R 2=0.086$ \\
\hline $\begin{array}{l}\text { Largest difference peak } \\
\text { and hole }\left(\mathrm{e} \cdot \AA^{3}\right)\end{array}$ & 0.465 and -0.537 & 0.499 and -0.347 & 0.440 and -0.275 & 0.647 and -0.559 \\
\hline
\end{tabular}


Table 2-3. Crystal data and structural refinement for $14,15,18$, and 19

\begin{tabular}{|c|c|c|c|c|}
\hline Compound & 14 & 15 & 18 & 19 \\
\hline Empirical formula & $\mathrm{C}_{22} \mathrm{H} 40 \mathrm{CuN}_{4} \mathrm{O}_{2} \mathrm{~S}_{2}$ & $\mathrm{C}_{12} \mathrm{H}_{20} \mathrm{CuN}_{4} \mathrm{O}_{2} \mathrm{~S}_{2}$ & $\mathrm{C}_{14} \mathrm{H}_{19} \mathrm{~N}_{5} \mathrm{OS}_{2}$ & $\mathrm{C}_{18} \mathrm{H}_{30} \mathrm{Cu}_{2} \mathrm{~N}_{10} \mathrm{O}_{2} \mathrm{~S}_{4}$ \\
\hline Formula weight & 520.24 & 379.98 & 337.46 & 673.84 \\
\hline Temperature (K) & $99.95(10)$ & $99.95(10)$ & $102.0(3)$ & $100.1(9)$ \\
\hline Wavelength $(\AA)$ & 0.71073 & 0.71073 & 0.71073 & 0.71073 \\
\hline Crystal system & Orthorhombic & Monoclinic & Monoclinic & Triclinic \\
\hline Space group & Pbcn & $C 2 / c$ & $P 2{ }_{1} / c$ & $P-1$ \\
\hline Unit cell dimension & $30.6050(7)$ & $20.267(2)$ & $9.91024(14)$ & $8.2759(4)$ \\
\hline $\mathrm{a}(\AA)$ & $9.10885(20)$ & $9.1509(9)$ & $16.6144(2)$ & $10.2427(3)$ \\
\hline $\mathrm{b}(\AA)$ & $9.19230(19)$ & $9.0767(10)$ & $20.7808(3)$ & $16.8758(6)$ \\
\hline c $(\AA)$ & 90 & 90 & 90 & $74.102(3)$ \\
\hline$\alpha\left(^{\circ}\right)$ & 90 & $102.350(12)$ & $96.2668(13)$ & $89.740(4)$ \\
\hline$\beta\left(^{\circ}\right)$ & 90 & 90 & 90 & $85.364(3)$ \\
\hline \multicolumn{5}{|l|}{$\gamma\left(\left(^{\circ}\right)\right.$} \\
\hline Volume $\left(\AA^{3}\right)$ & $2526.60(9)$ & $1644.4(3)$ & $3401.16(8)$ & 1371.07(9) \\
\hline $\mathrm{Z}$ & 4 & 4 & 8 & 2 \\
\hline$\rho_{\text {calcd. }}\left(\mathrm{Mg} / \mathrm{m}^{3}\right)$ & 1.348 & 1.535 & 1.318 & 1.632 \\
\hline Abs. coeff. $\left(\mathrm{mm}^{-1}\right)$ & 1.040 & 1.590 & 0.322 & 1.893 \\
\hline $\mathrm{F}(000)$ & 1108 & 788 & 1424 & 692 \\
\hline Crystal colour, habit & Orange needle & Orange plate & Yellow prism & Dark orange needle \\
\hline Crystal size $\left(\mathrm{mm}^{3}\right)$ & $0.37 \times 0.07 \times 0.04$ & $0.49 \times 0.07 \times 0.01$ & $0.40 \times 0.35 \times 0.33$ & $0.31 \times 0.05 \times 0.05$ \\
\hline $\begin{array}{l}\theta \text { range data collection } \\
\left({ }^{\circ}\right)\end{array}$ & 3.42 to $27.14^{\circ}$ & 3.21 to $28.16^{\circ}$ & 3.21 to 30.55 & 3.36 to 28.16 \\
\hline \multirow[t]{3}{*}{ Index ranges } & $-39 \leq \mathrm{h} \leq 39$ & $-26 \leq \mathrm{h} \leq 26$ & $-14 \leq \mathrm{h} \leq 14$ & $-10 \leq \mathrm{h} \leq 10$ \\
\hline & $-11 \leq \mathrm{k} \leq 11$ & $-12 \leq \mathrm{k} \leq 12$ & $-23 \leq \mathrm{k} \leq 23$ & $-13 \leq \mathrm{k} \leq 13$ \\
\hline & $-11 \leq 1 \leq 11$ & $-11 \leq 1 \leq 12$ & $-29 \leq 1 \leq 29$ & $-22 \leq 1 \leq 22$ \\
\hline Reflections collected & 35054 & $102 \overline{56}$ & 81797 & 25443 \\
\hline Independent & 2836 & 2003 & 10411 & 6693 \\
\hline reflections & {$[\mathrm{R}(\mathrm{int})=0.0382]$} & {$[\mathrm{R}(\mathrm{int})=0.0702]$} & {$[\mathrm{R}(\mathrm{int})=0.0416]$} & {$[\mathrm{R}(\mathrm{int})=0.0306]$} \\
\hline $\begin{array}{l}\text { Completeness to } \theta \\
\max \end{array}$ & $99.8 \%$ & $99.6 \%$ & $99.9 \%$ & $99.6 \%$ \\
\hline Absorption correction & Multi-scan & Multi-scan & Multi-scan & $\begin{array}{l}\text { Semi-empirical from } \\
\text { equivalents }\end{array}$ \\
\hline Max. and min. trans. & 1.000 and 0.871 & 1.000 and 0.577 & 1.000 and 0.970 & 1.000 and 0.673 \\
\hline Refinement method & $\begin{array}{l}\text { Full-matrix least- } \\
\text { squares on } \mathrm{F}^{2}\end{array}$ & $\begin{array}{l}\text { Full-matrix least- } \\
\text { squares on } \mathrm{F}^{2}\end{array}$ & $\begin{array}{l}\text { Full-matrix least- } \\
\text { squares on } \mathrm{F}^{2}\end{array}$ & $\begin{array}{l}\text { Full-matrix least- } \\
\text { squares on } \mathrm{F}^{2}\end{array}$ \\
\hline Data/restraints/params & $2836 / 0 / 221$ & $2003 / 0 / 136$ & $10411 / 0 / 483$ & $6693 / 0 / 445$ \\
\hline Goodness-of-fit on $\mathrm{F}^{2}$ & 1.013 & 1.085 & 1.036 & 1.039 \\
\hline $\begin{array}{lr}\text { Final } & R \\
{[\mathrm{I}>2 \sigma(\mathrm{I})]^{\mathrm{a}, \mathrm{b}}} & \text { indices }\end{array}$ & $\begin{array}{l}\mathrm{R} 1=0.0244 \\
\mathrm{wR} 2=0.0618\end{array}$ & $\begin{array}{l}\mathrm{R} 1=0.0398 \\
\mathrm{wR} 2=0.0937\end{array}$ & $\begin{array}{l}\mathrm{R} 1=0.387 \\
\mathrm{wR} 2=0.0825\end{array}$ & $\begin{array}{l}\mathrm{R} 1=0.0385 \\
\mathrm{wR} 2=0.0767\end{array}$ \\
\hline $\mathrm{R}$ indices (all data) & $\begin{array}{l}\mathrm{R} 1=0.0295 \\
\mathrm{wR} 2=0.0649\end{array}$ & $\begin{array}{l}\mathrm{R} 1=0.0562 \\
\mathrm{wR} 2=0.1059\end{array}$ & $\begin{array}{l}\mathrm{R} 1=0.0500 \\
\mathrm{wR} 2=0.0878\end{array}$ & $\begin{array}{l}\mathrm{R} 1=0.0486 \\
\mathrm{wR} 2=0.0821\end{array}$ \\
\hline $\begin{array}{l}\text { Largest difference } \\
\text { peak and hole }\left(\mathrm{e} \cdot \AA^{3}\right)\end{array}$ & 0.359 and -0.206 & 0.874 and -0.492 & 0.447 and -0.377 & 1.653 and -0.923 \\
\hline
\end{tabular}


Table 2-4. Crystal data and structural refinement for 21, 22, 28, and 29

\begin{tabular}{|c|c|c|c|c|}
\hline Compound & 21 & 22 & 28 & 29 \\
\hline Empirical formula & $\mathrm{C}_{28} \mathrm{H}_{34} \mathrm{Cu}_{2} \mathrm{~N}_{10} \mathrm{O}_{2} \mathrm{~S}_{4}$ & $\mathrm{C}_{9} \mathrm{H}_{15} \mathrm{~N}_{5} \mathrm{NiOS}_{2}$ & $\mathrm{C}_{10} \mathrm{H}_{18} \mathrm{~N}_{6} \mathrm{NiS}_{2}$ & $\mathrm{C}_{10} \mathrm{H}_{12} \mathrm{~F}_{6} \mathrm{~N}_{6} \mathrm{NiS}_{2}$ \\
\hline Formula weight & 797.97 & 332.09 & 345.13 & 453.09 \\
\hline Temperature $(\mathrm{K})$ & $100.2(6)$ & $102(3)$ & $100.2(6)$ & $100(2)$ \\
\hline Wavelength $(\AA)$ & 0.71073 & 0.71073 & 0.71073 & 0.71073 \\
\hline Crystal system & Triclinic & Monoclinic & Triclinic & Triclinic \\
\hline Space group & $P-1$ & $P 2{ }_{l} / c$ & $P-1$ & $P-1$ \\
\hline Unit cell dimension & $10.2767(5)$ & $7.7351(2)$ & $7.33437(16)$ & $8.3598(10)$ \\
\hline $\mathrm{a}\left(\AA \begin{array}{l}\AA \\
0\end{array}\right)$ & $11.8398(5)$ & $20.8045(6)$ & $8.5595(2)$ & $9.4027(10)$ \\
\hline $\mathrm{b}(\AA)$ & $14.8880(6)$ & $8.2308(3)$ & 11.6784 & $13.5112(17)$ \\
\hline $\mathrm{c}(\AA)$ & $73.776(4)$ & 90 & $103.865(2)$ & 109.467 \\
\hline$\alpha\left(^{\circ}\right)$ & $78850(4)$ & & $98.2102(19)$ & $105.475(11)$ \\
\hline$\beta\left(^{\circ}\right)$ & $\begin{array}{l}18.850(4) \\
75204(4)\end{array}$ & $\begin{array}{l}93.119(3) \\
90\end{array}$ & $91.7205(18)$ & $91.382(9)$ \\
\hline$\gamma\left({ }^{\circ}\right)$ & $75.204(4)$ & 90 & & \\
\hline Volume $\left(\AA^{3}\right)$ & $1667.09(13)$ & $1322.58(7)$ & $702.92(3)$ & $957.53(19)$ \\
\hline Z & 2 & 4 & 2 & 2 \\
\hline$\rho_{\text {calcd.. }}\left(\mathrm{Mg} / \mathrm{m}^{3}\right)$ & 1.590 & 1.668 & 1.631 & 1.571 \\
\hline Abs. coeff. $\left(\mathrm{mm}^{-1}\right)$ & 1.571 & 1.778 & 1.672 & 1.291 \\
\hline $\mathrm{F}(000)$ & 820 & 688 & 360 & 456 \\
\hline Crystal colour, habit & Orange plate & Thin orange plate & Orange prsim & Red-brown plate \\
\hline Crystal size $\left(\mathrm{mm}^{3}\right)$ & $0.31 \times 0.17 \times 0.03$ & $0.38 \times 0.05 \times 0.01$ & $0.40 \times 0.34 \times 0.18$ & $0.41 \times 0.08 \times 0.01$ \\
\hline $\begin{array}{l}\theta \text { range data collection } \\
\left({ }^{\circ}\right)\end{array}$ & 3.31 to 29.21 & 3.65 to 26.06 & 3.40 to 31.57 & 3.30 to 26.67 \\
\hline \multirow[t]{3}{*}{ Index ranges } & $-14 \leq \mathrm{h} \leq 14$ & $-9 \leq \mathrm{h} \leq 9$ & $-10 \leq \mathrm{h} \leq 10$ & $-10 \leq \mathrm{h} \leq 10$ \\
\hline & $-16 \leq \mathrm{k} \leq 16$ & $-25 \leq \mathrm{k} \leq 24$ & $-12 \leq \mathrm{k} \leq 12$ & $-11 \leq \mathrm{k} \leq 11$ \\
\hline & $-20 \leq 1 \leq 20$ & $-10 \leq 1 \leq 10$ & $-17 \leq 1 \leq 17$ & $-17 \leq 1 \leq 17$ \\
\hline Reflections collected & $441 \overline{48}$ & 11760 & 19998 & 13904 \\
\hline Independent & 8974 & 2601 & 4702 & 4031 \\
\hline reflections & {$[\mathrm{R}(\mathrm{int})=0.0373]$} & {$[\mathrm{R}(\mathrm{int})=0.0316]$} & {$[\mathrm{R}(\mathrm{int})=0.0217]$} & {$[\mathrm{R}(\mathrm{int})=0.058]$} \\
\hline $\begin{array}{l}\text { Completeness to } \theta \\
\max \end{array}$ & $99.2 \%$ & $99.4 \%$ & $99.9 \%$ & $99.7 \%$ \\
\hline Absorption correction & $\begin{array}{l}\text { Semi-empirical } \\
\text { from equivalents }\end{array}$ & Multi-scan & Multiscan & Multiscan \\
\hline Max. and min. trans. & 1.000 and 0.792 & 1.000 and 0.939 & 1.000 and 0.778 & 1.00 and 0.678 \\
\hline Refinement method & $\begin{array}{l}\text { Full-matrix least- } \\
\text { squares on } \mathrm{F}^{2}\end{array}$ & $\begin{array}{l}\text { Full-matrix least- } \\
\text { squares on } \mathrm{F}^{2}\end{array}$ & $\begin{array}{l}\text { Full-matrix least- } \\
\text { squares on } \mathrm{F}^{2}\end{array}$ & $\begin{array}{l}\text { Full-matrix least- } \\
\text { squares on } \mathrm{F}^{2}\end{array}$ \\
\hline Data/restraints/params & $8974 / 0 / 429$ & $2601 / 1 / 179$ & $4702 / 0 / 244$ & $4031 / 0 / 288$ \\
\hline Goodness-of-fit- on $\mathrm{F}^{2}$ & 1.038 & 1.071 & 1.013 & 1.074 \\
\hline $\begin{array}{l}\text { Final R indices } \\
{[\mathrm{I}>2 \sigma(\mathrm{I})]^{\mathrm{a}, \mathrm{b}}}\end{array}$ & $\begin{array}{l}\mathrm{R} 1=0.028, \\
\mathrm{wR} 2=0.0618\end{array}$ & $\begin{array}{l}\mathrm{R} 1=0.0353, \\
\mathrm{wR} 2=0.0823\end{array}$ & $\begin{array}{l}\mathrm{R} 1=0.0196 \\
\mathrm{wR} 2=0.0533\end{array}$ & $\begin{array}{l}\mathrm{R} 1=0.0500 \\
\mathrm{wR} 2=0.1099\end{array}$ \\
\hline $\mathrm{R}$ indices (all data) & $\begin{array}{l}\mathrm{R} 1=0.0355, \\
\mathrm{wR} 2=0.0655\end{array}$ & $\begin{array}{l}\mathrm{R} 1=0.0405 \\
\mathrm{wR} 2=0.0846\end{array}$ & $\begin{array}{l}\mathrm{R} 1=0.0211 \\
\mathrm{wR} 2=0.0545\end{array}$ & $\begin{array}{l}\mathrm{R} 1=0.0647 \\
\mathrm{wR} 2=0.1188\end{array}$ \\
\hline $\begin{array}{l}\text { Largest difference } \\
\text { peak and hole }\left(e \cdot \AA^{3}\right)\end{array}$ & 0.843 and -0.466 & 0.619 and -0.301 & 0.525 and -0.285 & 1.176 and -0.477 \\
\hline
\end{tabular}




\subsection{Preparation of Modified Glassy Carbon Electrodes}

Complexes 2, 28, and 29 (4 mg each) were dispersed in $1 \mathrm{~mL}$ acetonitrile (VWR, ACS grade, dried using an MB-SPS from MBRAUN) using a vortex mixer (Vortex Genie 2 , Scientific Industries). The $10 \%(\mathrm{v} / \mathrm{v})$ aqueous nafion solution $(12.5 \mu \mathrm{L})$ was then added to the resulting ink. The dispersion was further homogenized via ultrasonication (ColeParmer Ultrasonic Bath) for $2 \mathrm{~h}$. After sonication, $10 \mu \mathrm{L}$ of the resulting dispersion was dropped onto a glassy carbon electrode (E4TQ ChangeDisk, Pine Research), rotating at 50 RPM, affixed to a rotator (MSR Rotator, Pine Research). The rotation speed was subsequently increased to 300 RPM, and this speed was maintained until the film dried.

\subsection{Electrode Surface Characterization}

Scanning electron microscopy (SEM) was carried out using a TESCAN VEGA3 microscope operating at $10 \mathrm{kV}$ and $10 \mathrm{~mA}$. Images of the catalyst materials were taken using the ejectable glassy carbon electrode disk tip as the substrate. As above, catalyst dispersion was dropped onto the glassy carbon electrode and allowed to dry over rotation. At this point, either the disk was ejected and taken to the SEM, or the material was cycled to peak activity (as described above) before disk ejection and characterization. Raman spectroscopy was carried out using a Renishaw inVia spectrometer with red (632 nm) laser

(Renishaw RL 633) operating at $100 \%$ power. Samples were prepared for Raman as described for SEM.

\subsection{MTT Assay}

MTT assays were performed by Dr. Sarah Andres at the University of Louisville medical school in collaboration with Dr. Paula Bates. Anti-proliferative activity of 16-27 were evaluated using a previously published 3-(4,5-dimethylthiazol-2-yl)-2,5- 
diphenyltetrazolium bromide (MTT) assay protocol. ${ }^{88-90}$ Cells were seeded in quadruplicate wells in 96-well plates and allowed to adhere overnight. To account for intrinsic differences in growth rates, cells were plated at the following densities to achieve comparable MTT absorbance values (OD570 between 0.5 and 1) for untreated cells: A549, 1000 cells/well; IMR-90, 5000 cells/well. After $72 \mathrm{~h}$ of treatment with test compounds, MTT (Sigma/Millipore Sigma, St. Louis, MO) was added for $4 \mathrm{~h}$ prior to cell lysis. Each assay was performed in at least triplicate. All readings were normalized to the vehicle treatment of only DMSO. 


\section{CHAPTER III}

\section{SYNTHESES, STRUCTURES, AND ELECTROCHEMICAL STUDIES OF $N, N{ }^{\prime}-$ BIS(ALKYLTHIOCARBAMATE)BUTANE-2,3-DIIMINE CU(II) COMPLEXES AS PENDANT ALKOXY DERIVATIVES OF CU(ATSM)}

\subsection{Introduction}

The biological applications of $\mathbf{1}$ as a diagnostic and therapeutic agent have been extensively studied. ${ }^{22-31,38} \mathrm{The} \mathrm{Cu}(\mathrm{II})$ ion sits in a conjugated dianionic $\mathrm{N}_{2} \mathrm{~S}_{2}$ framework with terminal -NHMe functional groups that do not participate in metal-ligand binding. The key structural and electronic facets of $\mathbf{1}$ that make it ideal for biological studies are its neutrality, planarity, and reduction potential. The rigid planarity of $\mathbf{1}$ is exemplified by the best fit plane for all 17 non-hydrogen atoms, for which the largest deviation $\pm 0.047 \AA$ for $\mathrm{N} 2 / \mathrm{N} 2{ }^{\prime}$ with a standard deviation of $\pm 0.025 \AA .{ }^{91}$ Along with the planarity, the complex is neutral and is capable of diffusing across cell membranes and is only retained by gaining a negative charge upon reduction. Compound $\mathbf{1}$ can therefore freely diffuse across cell membranes to either enter or exit a cell under normoxic conditions. However, under the low oxygen tension of hypoxia, $\mathbf{1}$ is reduced in the cytosol and thus preventing it from exiting the cell, Figure 3-1. This redox selectivity of $\mathbf{1}$ has led to its evaluation as a ${ }^{64} \mathrm{Cu}$ and ${ }^{62} \mathrm{Cu}$ radiopharmaceutical for selective imaging of hypoxia. ${ }^{27-31}$ 
A

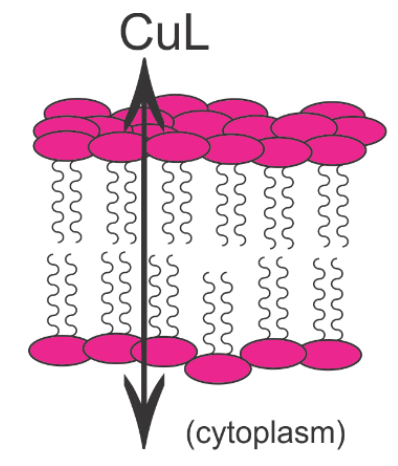

CuL

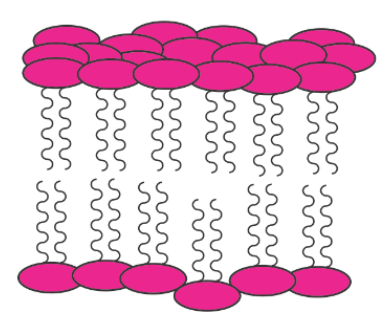

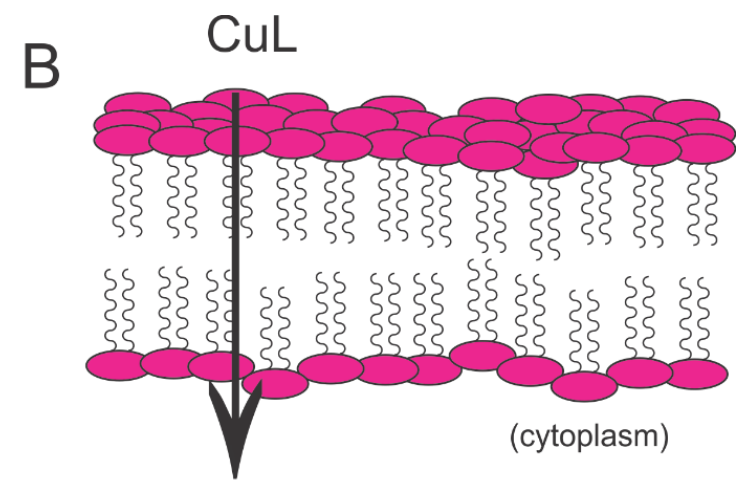
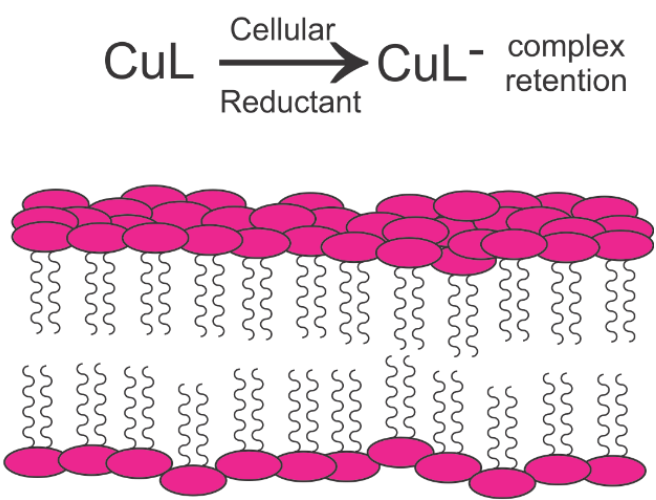

Figure 3-1. Depiction of copper retention in normoxic (A) and hypoxic (B) cells.

Further, 1 has been evaluated for the treatment of neurodegenerative diseases. ${ }^{22-25}$

Phase I clinical trials, as a therapeutic for amyotrophic lateral sclerosis/motor neuron disease (ALS/MND) are currently underway. ${ }^{26}$ The redox selectivity is further exemplified by comparison of 1 with the copper complexes of PTSM $(+80 \mathrm{mV})$ and GTSM $(+160 \mathrm{mV})$, Figure 3-2. The CuPTSM derivative with its more anodic $\mathrm{Cu}^{\mathrm{II} / \mathrm{I}}$ couple was found to not be retained in cells in a discriminating manner when compared to $\mathbf{1}$ for imaging. In therapeutics, the treatment of cancer cells have been evaluated comparing 1 with CuGTSM. Here however, it was found that $\mathbf{1}$ was ineffective in the treatment of cancer cells. ${ }^{38}$
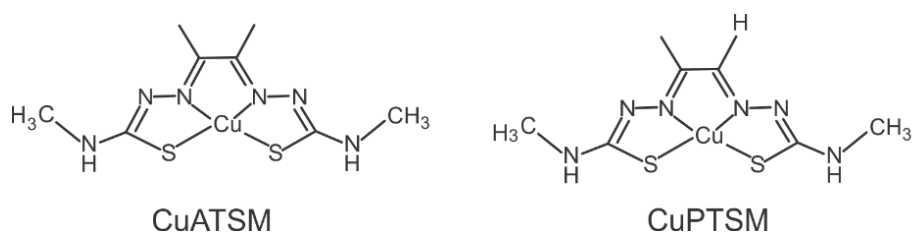

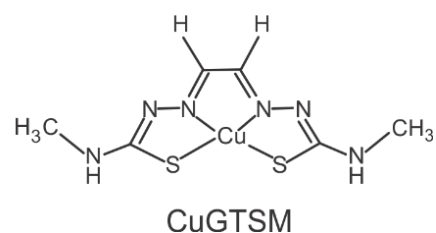

CuGTSM

Figure 3-2. Structural comparison of CuATSM, CuPTSM, and CuGTSM. 
The biological importance of $\mathbf{1}$ has led to the synthesis and characterization of a large number of related copper bis(thiosemicarbazonato) complexes with symmetric and asymmetric variation at the $\mathrm{R}_{1}$ and $\mathrm{R}_{2}$ positions, shown in Scheme 3-1.7,13,15,19,21,32-33,39-40,92 Substitution at the $\mathrm{R}_{1}$ position has largely focused on other amine derivatives to investigate the impact on selectivity and/or reduction potential. In general, chain extension has a small effect on the $\mathrm{Cu}^{\mathrm{II} / \mathrm{I}}$ potential. ${ }^{13,15}$ However, modification with aromatic rings can lead to stabilization of $\mathrm{Cu}(\mathrm{I})$ by as much as $+240 \mathrm{mV} .{ }^{39}$ Que and co-workers recently reported symmetric and asymmetric CuATSM derivatives with the -NHMe groups replaced by $\mathrm{NHCH}_{2} \mathrm{CF}_{3}$ with stabilization of $\mathrm{Cu}(\mathrm{I})$ by $\sim 70 \mathrm{mV}$ per $-\mathrm{NHCH}_{2} \mathrm{CF}_{3}$. Interestingly, these complexes allow for hypoxia imaging by ${ }^{19} \mathrm{~F}$ MRI, thus avoiding the need for ${ }^{64} \mathrm{Cu} \cdot{ }^{32}$ Variation of the $\mathrm{R}_{2}$ backbone methyl groups with hydrogen or phenyl substituents also stabilize $\mathrm{Cu}(\mathrm{I})$, with the later showing a larger effect of $\sim 240 \mathrm{mV}$. ${ }^{13,39}$ The effect of $\mathrm{R}_{2}$ group substitution for a wide variety of functional groups was evaluated using experimentally benchmarked density functional theory calculations of $\mathrm{Cu}^{\mathrm{II} / \mathrm{I}}$ reduction potentials. A Hammett analysis of the theoretical $\mathrm{Cu}^{\mathrm{II} / \mathrm{I}}$ potentials is consistent with an inductive effect where $\mathrm{Cu}(\mathrm{I})$ is stabilized by the electron-withdrawing substituents. ${ }^{16}$

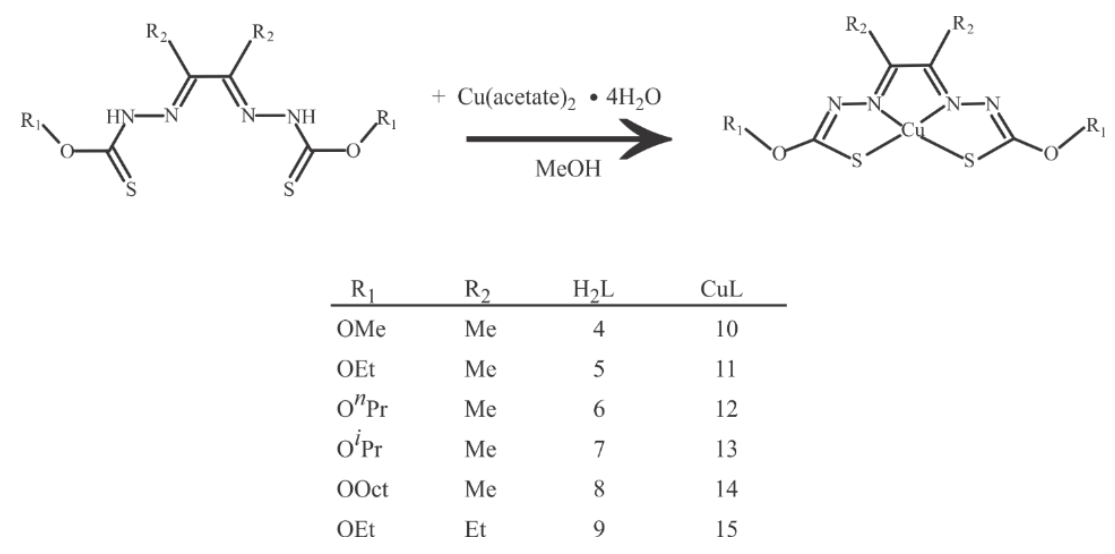

Scheme 3-1. Synthesis of compounds 10-15. 
The numerous derivatives of $\mathrm{Cu}(\mathrm{ATSM})$ reported to date have by and large maintained an $\mathrm{N}_{2} \mathrm{~S}_{2}$ donor core with terminal amine based $\mathrm{R}_{1}$ groups. The research in this dissertation highlights a modification to the ligand framework by incorporation of alkoxy groups at the terminal $\mathrm{R}_{1}$ position. As outlined in Scheme 3-1, a series of six ligands and their $\mathrm{Cu}(\mathrm{II})$ complexes have been synthesized and fully characterized. One compound was previously synthesized and characterized by UV-visible spectroscopy. ${ }^{93}$ As detailed below, this results in a substantial shift in the $\mathrm{Cu}(\mathrm{II} / \mathrm{I})$ reduction potential relative to $\mathbf{1}$.

\subsection{Results}

\subsubsection{Synthesis and Characterization}

A series of $\mathrm{N}_{2} \mathrm{~S}_{2} N, N^{\prime}$-bis(alkylthiocarbamate)butane-2,3-diimine ligands (4 - 8) with varying $O$-alkyl chain lengths and $N, N^{\prime}$-bis(methylthiocarbamate)hexane-3,4-diimine (9) have been prepared and complexed with $\mathrm{Cu}(\mathrm{II})$ yielding the square planar complexes 10 - 15. The ligands were obtained in three steps from inexpensive bulk commodity chemicals, Scheme 3-2.
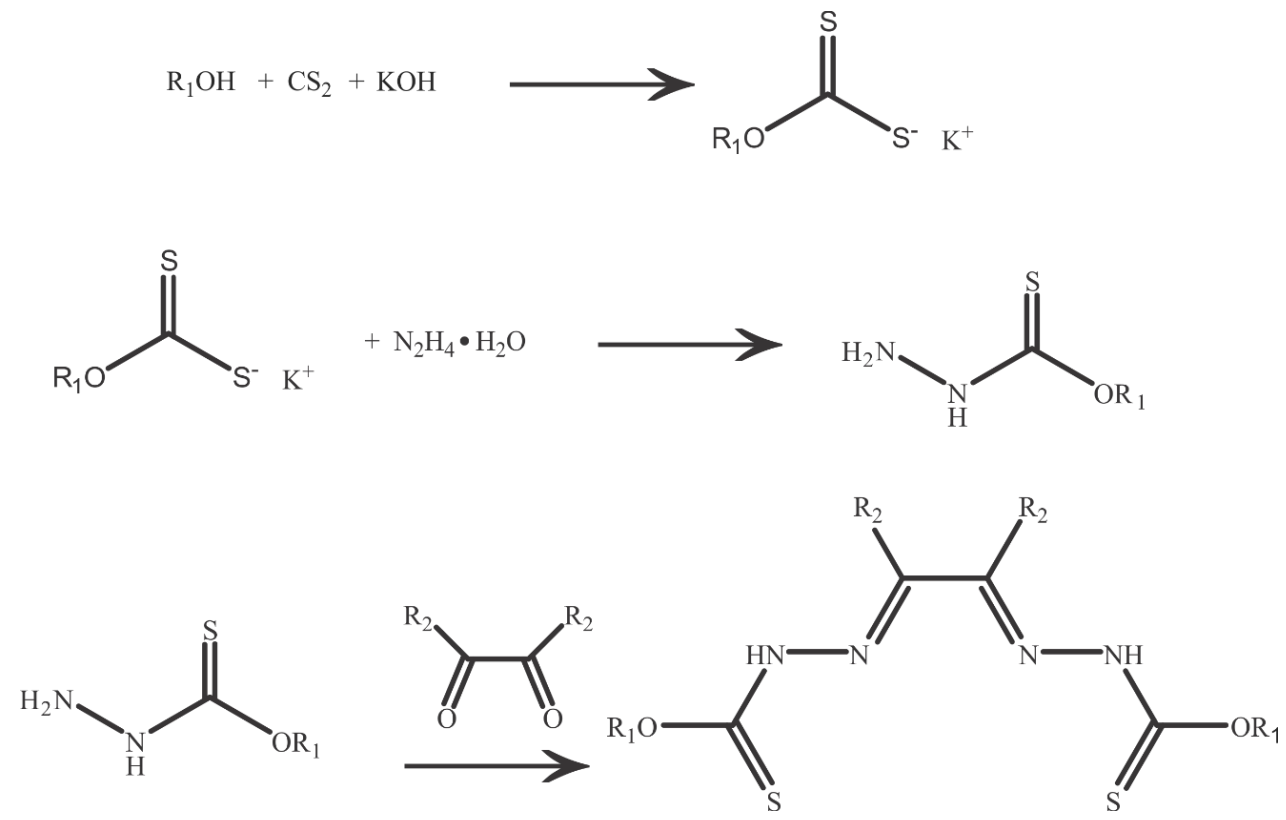

Scheme 3-2. Synthesis of ligands 4-9. 
Using previously reported methods, xanthates were prepared from the appropriate alcohol and carbon disulfide. ${ }^{79}$ The xanthates were subsequently condensed with hydrazine monohydrate to yield the hydrazinecarbothioc acid $O$-alkyl esters. ${ }^{80}$ Condensation of the ester with butanedione $(\mathbf{4}-\mathbf{8})$ or hexanedione (9) yielded the desired ligand in a reaction similar to $\mathrm{H}_{2} \mathrm{ATSM}$ synthesis. ${ }^{14}$ Notably, all ligand syntheses were conducted open to the atmosphere in ethanol solution with facile purification by filtration and washing. The simplicity of these methods and the variety of available alcohols and diones make $N, N^{\prime}-$ bis(alkylthiocarbamate)butane-2,3-diimine and its derivatives potentially useful new class of ligands related to the bis-thiosemicarbazone family.

The ${ }^{1} \mathrm{H}$ NMR of 4, seen in Figure 3-3, displays a prototypical chemical shift for methoxy protons at $\delta 4.03$. This chemical shift is a key identifier for this ligand class of the protons adjacent to the oxygen near 4.00. The backbone protons occur at $\delta 2.11$, which is another indication of condensation. A unique feature for this subset of ligands is the two chemical shifts at $\delta 11.46$ and 11.77 , corresponding to the imino hydrogen. BTSC analogs display only one peak for this hydrogen. This unique pattern could potentially arise from tautomerism, however temperature dependent NMR studies were not conducted. The ${ }^{13} \mathrm{C}$ NMR of 4, Figure 3-4, shows prototypical peaks of tertiary $\mathrm{C}=\mathrm{N}$ and $\mathrm{C}=\mathrm{S}$ carbons at $\delta$ 117.8 and 188.0, respectively. The methyl carbons of the backbone and alkoxy functionality are present at $\delta 58.5$ and 12.3 respectively. The remaining ${ }^{1} \mathrm{H}$ and ${ }^{13} \mathrm{C}$ NMR spectra for 5-9 can be found in the appendix, Figures A1-A9. 


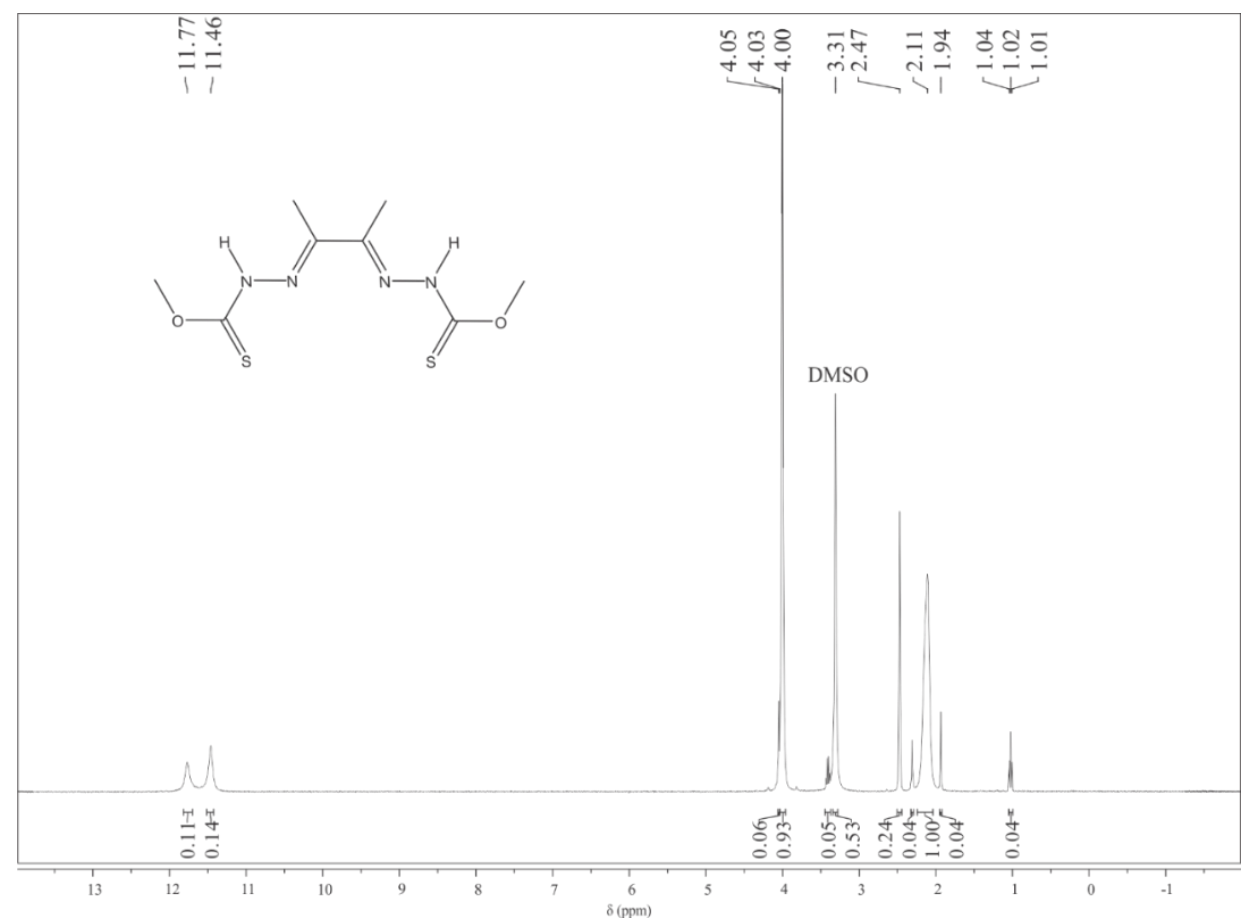

Figure 3-3. ${ }^{1} \mathrm{H}$ NMR of 4 in DMSO.

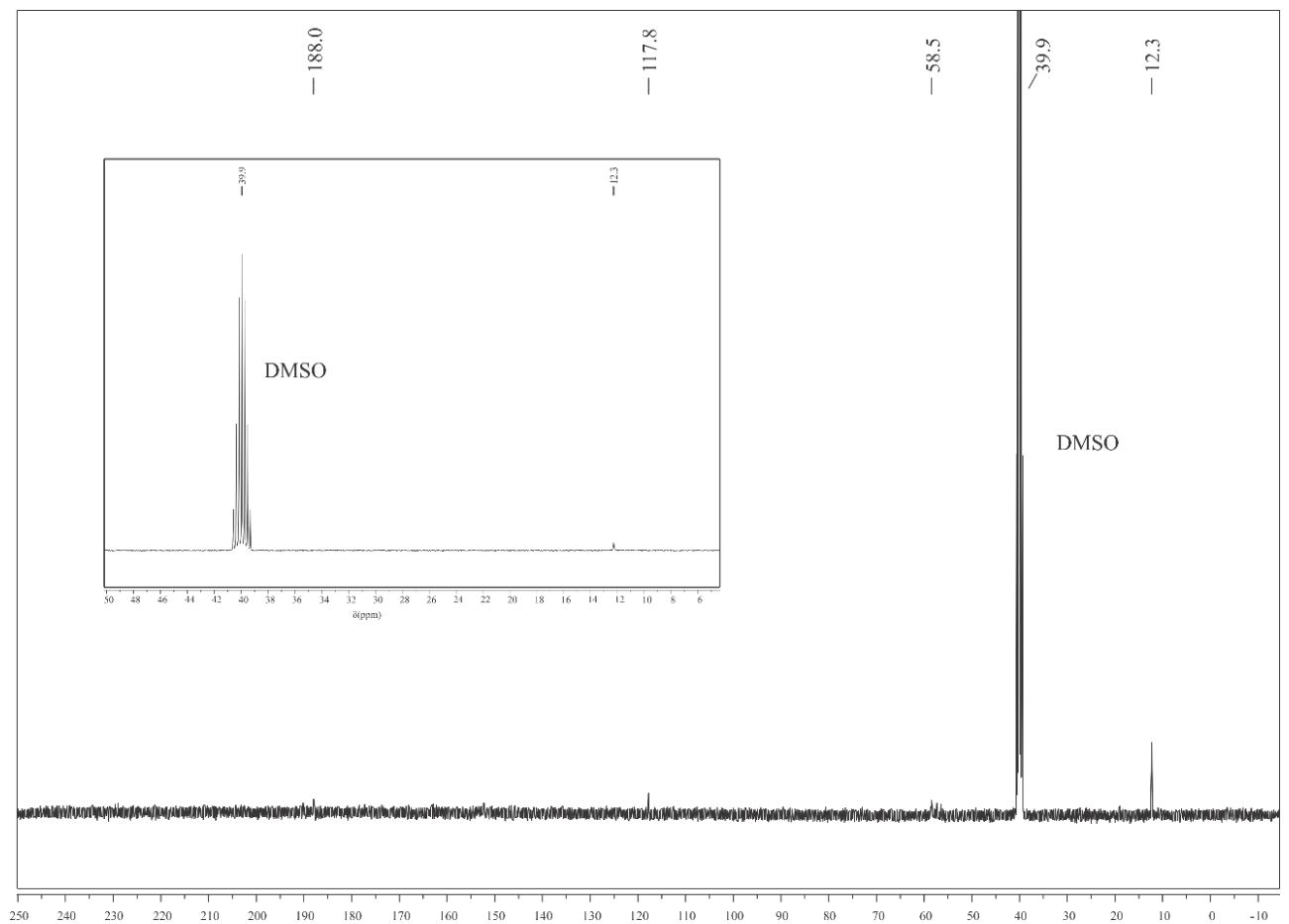

Figure 3-4. ${ }^{13} \mathrm{C}$ NMR of 4 in DMSO, with the ppm inset of a zoomed out image to display the DMSO peak. 
The FT-IR spectrum of 4, Figure 3-5A, is representative for this class of compounds. Key stretches are the $\mathrm{N}-\mathrm{H}, \mathrm{C}-\mathrm{O}$, and $\mathrm{C}=\mathrm{S}$. The $\mathrm{C}-\mathrm{O}$ and $\mathrm{C}=\mathrm{S}$ occur at 1137 and $1060 \mathrm{~cm}^{-1}$ in $\mathbf{4}$. The $\mathrm{N}-\mathrm{H}$ stretch, at 3240 for $\mathbf{4}$, is paramount in identification. The corresponding hydrazinecarbothioc acid $O$-alkyl ester, has a primary and secondary amine, thus has multiple $\mathrm{N}-\mathrm{H}$ stretches that are not present in the ligand. Further, the IR spectra of 10-15 display no $\mathrm{N}-\mathrm{H}$ stretching, indicative of deprotonation, Figure 3-5B. The loss of these protons generates a $2^{-}$charge on the ligand, in turn creating a neutral chelate from a metal $2^{+}$ion. Therefore, to generate a neutral complex, a suitable base is needed to deprotonate the ligand framework. A suitable metal salt may contain a basic counter ion (i.e., acetate) or an exogenous base may be used. Further, the $\mathrm{C}-\mathrm{O}$ and $\mathrm{C}=\mathrm{S}$ stretches shift to 1091 and $948 \mathrm{~cm}^{-1}$, respectively. Lastly, upon metalation, new peaks occur in the IR spectrum at 831 and $671 \mathrm{~cm}^{-1}$ that indicate $\mathrm{Cu}-\mathrm{S}$ and $\mathrm{Cu}-\mathrm{N}$ stretching. The remaining FTIR spectra can be found in the Appendix (Figures A10-A19).
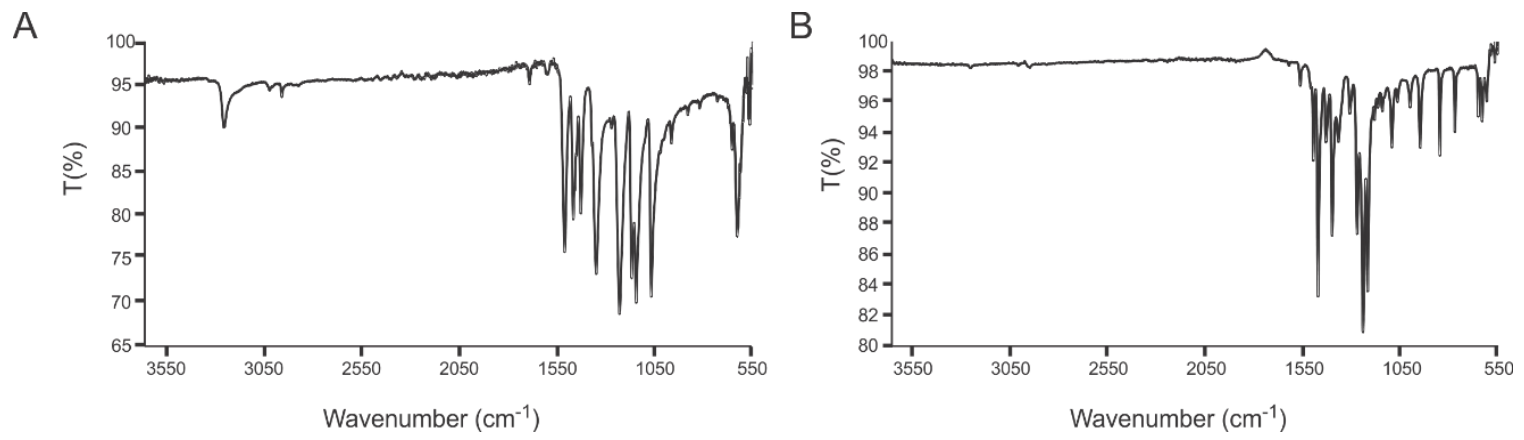

Figure 3-5. FT-IR spectra of 4 (A) and its copper complex 10 (B).

The electronic spectra of $\mathbf{1 0}$ - $\mathbf{1 5}$ recorded in dichloromethane display a ligand to metal charge transfer band near $485 \mathrm{~nm}$ and two more intense ligand to ligand charge transfer bands near 248 and $290 \mathrm{~nm}$. The spectrum of $\mathbf{1 0}$ is shown in Figure 3-6 as a representative of the whole class of compounds. The spectra of 11-15 can be found in the Appendix (Figures A20-A24). The transition energies are largely insensitive to the identity 
of the $O$-alkyl group $\mathrm{R}_{1}$ or the backbone substituent $\mathrm{R}_{2}$, Table 3-1. As shown in Table 31, the high energy bands for $\mathbf{1 0}$ at 246 and $290 \mathrm{~nm}$ are red shifted by 2340 and $2640 \mathrm{~cm}^{-1}$ with respect to the bands at 261 and $314 \mathrm{~nm}$ in $\mathrm{Cu}(\mathrm{ATSM}), \mathbf{1}$. The lower energy band of $\mathbf{1 0}$ at $486 \mathrm{~nm}$ is blue shifted by $388 \mathrm{~cm}^{-1}$ relative to the band at $477 \mathrm{~nm}$ in Cu(ATSM). ${ }^{13}$ The shifts are attributed to electronic differences within the ligand framework and no solvent effects. The spectra of $\mathbf{1 0}$ in DCM and DMSO are not substantially different.

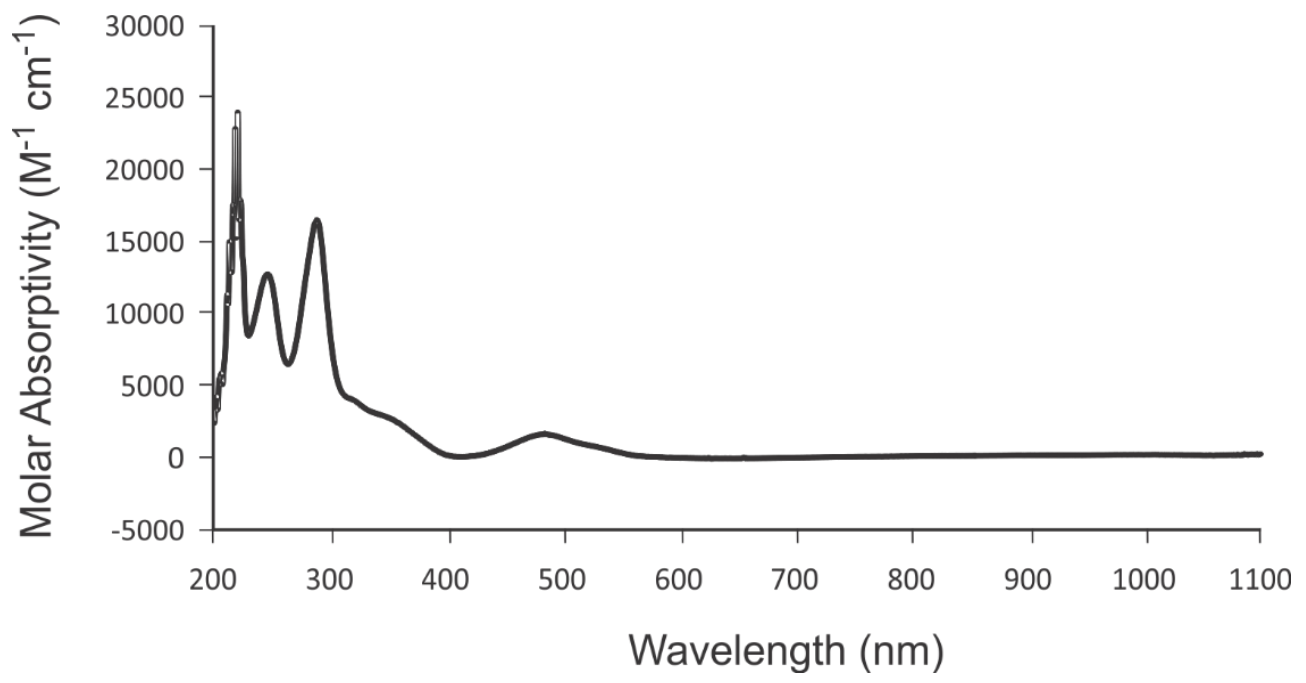

Figure 3-6. UV-visible spectrum of 10, representative of the class.

The EPR spectra of $\mathbf{1 0}-\mathbf{1 5}$ were recorded as powders at room temperature. Complexes 10 - 15 are expected to be square planar with an unpaired electron in the $d_{x 2-y 2}$ orbital. The unpaired electron could undergo transitions to the $d_{x y}, d_{x z}$, and $d_{y z}$ orbitals. Since the $d_{x z}$ and $d_{y z}$ are degenerate in a square planar environment, only two transitions are expected to be observed with the $d_{x z / y z}$ transition twice the intensity. As shown in Figure 3-7 complex 12 displays an axial spectrum with $\mathrm{g}_{\|}>\mathrm{g}_{\perp}>2$ (Table 3-1) consistent with square planar $\mathrm{Cu}(\mathrm{II})$ and a single unpaired electron located in $\mathrm{d}_{\mathrm{x} 2-\mathrm{y} 2}$. The remaining spectra can be found in the Appendix (Figures A25-A29). Simulated values for $g_{\|}$and $g_{\perp}$ fall in 
the narrow ranges of $2.031-2.042$ and $2.089-2.131$, respectively, consistent with the similarities observed for $\mathbf{1 1}-\mathbf{1 5}$ in the electronic spectrum. The spectrum of $\mathbf{1 0}$ is too broad to resolve the axial signal, but the observed g-value of 2.061 is near the average g-values of the other complexes.

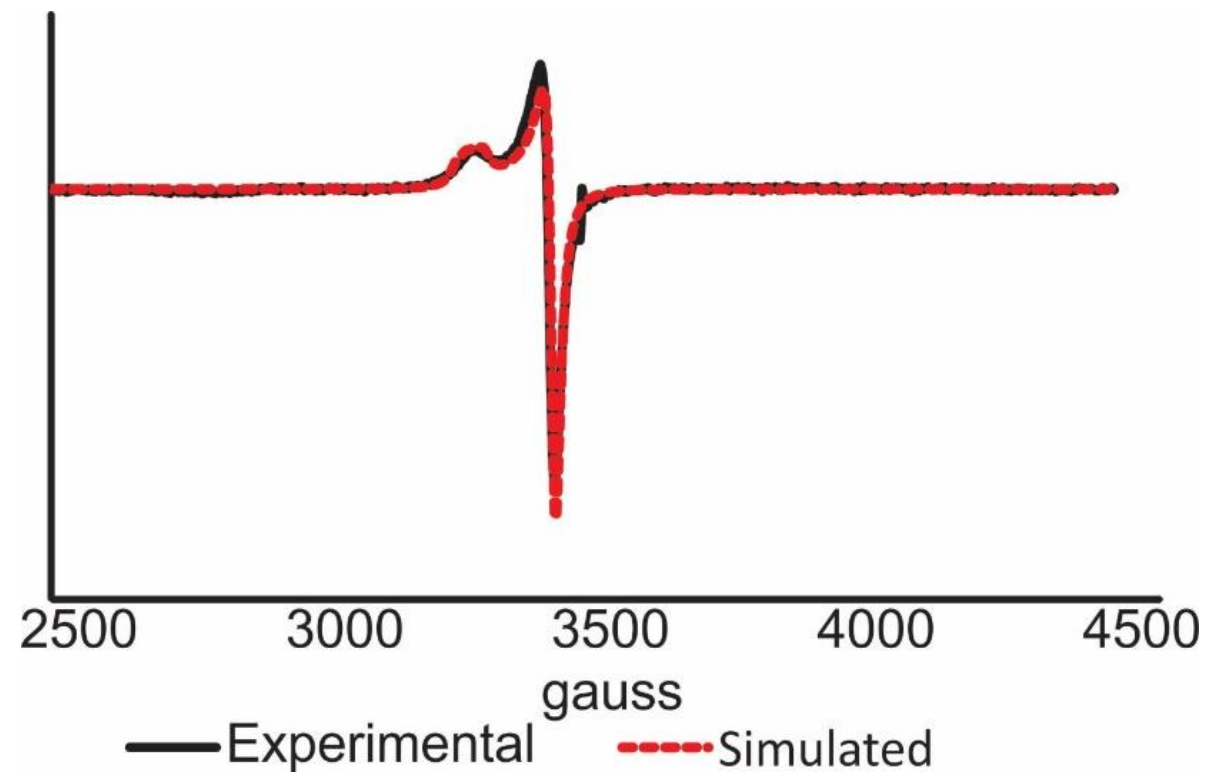

Figure 3-7. Experimental (solid black line) and simulated (dashed red line) EPR spectrum of 12. This spectrum is representative of the whole class of compounds.

A comparison of the EPR and UV-visible data shows only small changes in the spectroscopic features of complexes $\mathbf{1 0}$ - $\mathbf{1 5}$ as compared to similar bis-thiosemicarbazone analogues, $\mathbf{1}$ and $\mathbf{3 0}-\mathbf{3 3}$. The axial EPR spectra of $\mathbf{1}, \mathrm{Cu}(\mathrm{ATSM})$, is indistinguishable from those of $11-\mathbf{1 5}$. The reported $g_{\|}$and $g_{\perp}$ values of 2.115 and 2.038 for $\mathbf{1}$ fall within the narrow ranges of $2.031-2.042$ and $2.089-2.131$ for 11 to 15 , Table $3-1$, indicating no significant changes in the relative energies of the d-orbital manifold.

\subsubsection{Electrochemical Studies}

The cyclic voltammograms of $\mathbf{1 0}-\mathbf{1 5}$ in dichloromethane, each show two reversible events assigned to $\mathrm{Cu}^{\mathrm{III} / \mathrm{II}}$ and $\mathrm{Cu}^{\mathrm{II} / \mathrm{I}}$ couples, Table $3-1$. The $\mathrm{E}_{1 / 2}$ values for the 
$\mathrm{Cu}^{\mathrm{III} / \mathrm{II}}$ and $\mathrm{Cu}^{\mathrm{II} / \mathrm{I}}$ events of $\mathbf{1 0}$ are observed at +585 and $-857 \mathrm{mV}$ versus ferrocenium/ferrocene, respectively, Figure 3-8. The remaining voltammograms can ebe found in the Appendix (Figures A30-A34). Variation of the side-chain $\mathrm{R}_{1}$ and backbone $\mathrm{R}_{2}$ groups modulates the $\mathrm{Cu}^{\mathrm{I} / \mathrm{I}}$ reduction potential. Increasing the length of the $\mathrm{R}_{1}$ from OMe to $-\mathrm{OEt}$ and $-\mathrm{O}^{n} \mathrm{Pr}$ systematically decreases the $\mathrm{Cu}^{\mathrm{II} / \mathrm{I}}$ potential of $\mathbf{1 1}$ and $\mathbf{1 2}$ to -879 and $-892 \mathrm{mV}$, respectively. Further chain extension to -O-octyl, 14, slightly increases the potential to $-884 \mathrm{mV}$. Substitution of $-\mathrm{O}^{n} \operatorname{Pr}$ with $-\mathrm{O}^{i} \operatorname{Pr}$ further destabilizes $\mathrm{Cu}(\mathrm{I})$ as indicated by the $\mathrm{Cu}^{\mathrm{II} / \mathrm{I}}$ potential of $\mathbf{1 3}$ at $-904 \mathrm{mV}$. Changes at the $\mathrm{R}_{2}$ slightly influence the reduction potential as shown by a comparison of $\mathbf{1 1}$ and $\mathbf{1 5}$, with the former more difficult to reduce by $5 \mathrm{mV}$.

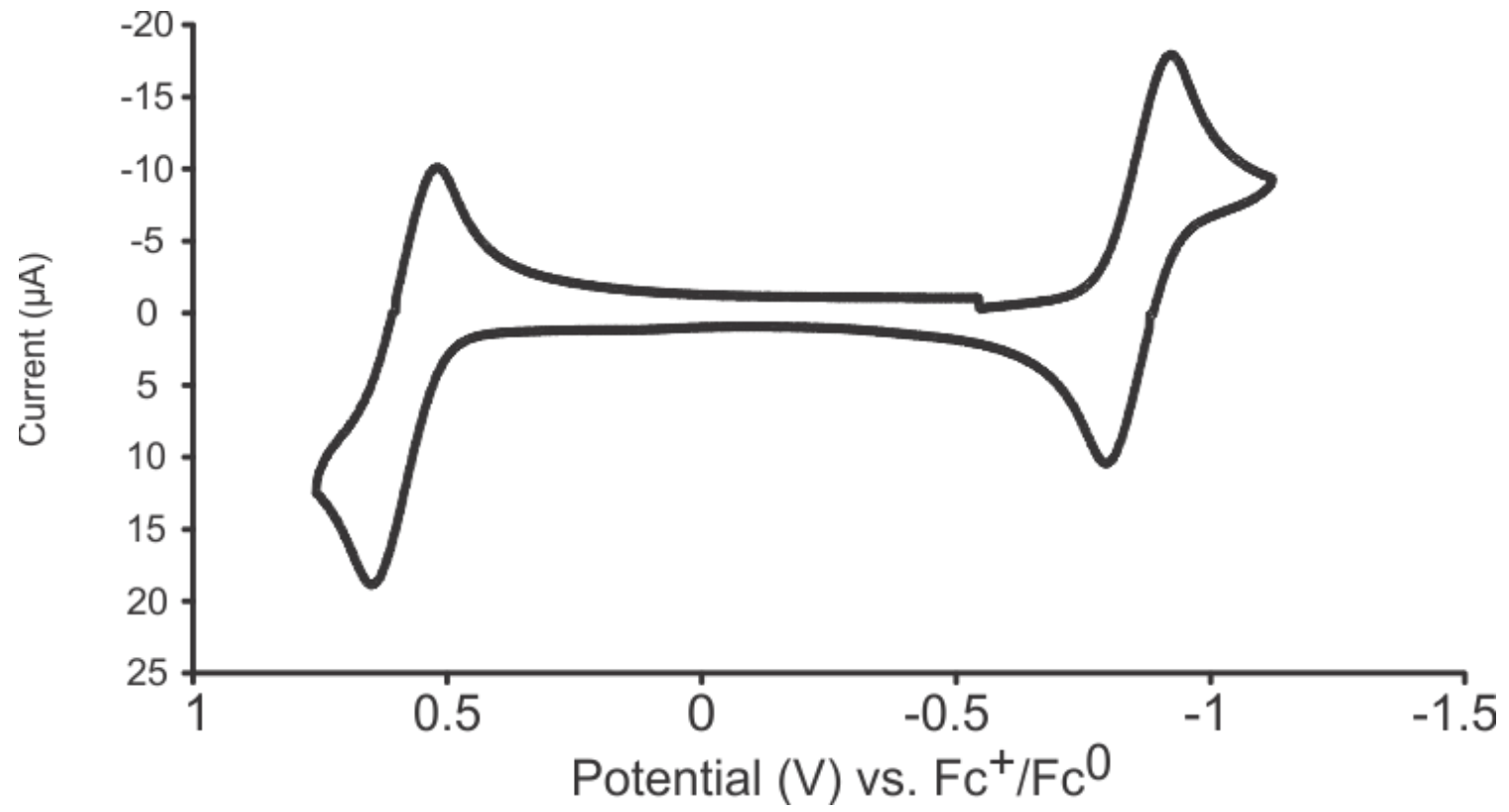

Figure 3-8. Cyclic voltammogram of $\mathbf{1 0}$ in $\mathrm{CH}_{2} \mathrm{Cl}_{2}$ with $0.1 \mathrm{M}$ tetrabutylammonium hexafluorophosphate as supporting electrolyte. Scan rate $=100 \mathrm{mV} / \mathrm{s}$; potentials referenced versus $\mathrm{Fc}^{+} / \mathrm{Fc}$. 
A comparison of the methylamine derivative $\mathbf{1}$ with the methoxy derivative $\mathbf{1 0}$ shows a $+253 \mathrm{mV}$ shift in the $\mathrm{Cu}^{\mathrm{II} / \mathrm{I}}$ reduction potential. A similar shift of $+241 \mathrm{mV}$ is observed upon substitution of -NHEt (34) with -OEt (11). These large shifts can be attributed to an inductive effect consistent with the electron withdrawing properties of the alkoxides and prior Hammet analysis of the -NHR functional group effect on reduction potentials. ${ }^{16}$ Extension of the carbon chain in the $\mathrm{R}_{2}$ position from Me to Et results in a 5 $\mathrm{mV}$ increase from compound $\mathbf{1 1}$ to $\mathbf{1 5}$, whereas the related bis-thiosemicarbazone analogues $\mathbf{1}$ and $\mathbf{3 6}$ show an increase of $10 \mathrm{mV}$ confirming the new class of compounds follow the same electrochemical trend. This difference in reduction potential would indicate that this new class of compounds would not be ideal for imaging given the ease of accessibility. However in comparison with $\mathbf{3 5}$, which as previously mentioned has potential therapeutic properties, this new class of compounds has a more anodic $\mathrm{Cu}^{\mathrm{II/I}}$ potential by approximately $100 \mathrm{mV}$. Thus, these compounds could be useful in the treatment of cancer. Unfortunately, these compounds are only soluble in DCM, which is not suitable for biological studies. In total, six new complexes with $\mathrm{Cu}^{\mathrm{II/I}}$ reduction potentials in the range of $-904 \mathrm{mV}$ to $-857 \mathrm{mV}$ have been prepared demonstrating that substitution of $-\mathrm{NHR}$ with $-\mathrm{OR}$ yields a large anodic shift in the $\mathrm{Cu}^{(\mathrm{II} / \mathrm{I})}$. 


\begin{tabular}{|c|c|c|c|c|c|c|c|}
\hline Complex & $\mathrm{R}_{1}$ & $\mathrm{R}_{2}$ & $\begin{array}{c}\lambda_{\max }^{a} \\
(\mathrm{~nm})(\varepsilon)\end{array}$ & $\begin{array}{l}g_{\|}, g_{\perp} \\
\left(g_{\text {avg }}\right)^{\mathrm{b}}\end{array}$ & $\begin{array}{c}\mathrm{Cu}^{\mathrm{III} / I \mathrm{II}} \\
E_{1 / 2}\left(i_{p c} / i_{p a}\right) \\
(\mathrm{mV})^{\mathrm{c}}\end{array}$ & $\begin{array}{c}\mathrm{Cu}^{\mathrm{II} / \mathrm{I}} \\
E_{1 / 2}\left(i_{p c} / i_{p a}\right) \\
(\mathrm{mV})^{\mathrm{c}}\end{array}$ & Ref. \\
\hline 10 & $\mathrm{OMe}$ & $\mathrm{Me}$ & $\begin{array}{c}246(16,000) 290(20,000) \\
486(2,680)\end{array}$ & $(2.061)$ & $585(0.934)$ & $-857(0.941)$ & 77 \\
\hline 11 & OEt & $\mathrm{Me}$ & $\begin{array}{c}248(16,300) 290(22,700) \\
485(2,920)\end{array}$ & $2.131,2.036(2.068)$ & $528(0.933)$ & $-879(0.965)$ & 77 \\
\hline 12 & $\mathrm{O}^{n} \mathrm{Pr}$ & $\mathrm{Me}$ & $\begin{array}{c}248(16,600) 291(23,800) \\
484(2,930)\end{array}$ & $2.127,2.034(2.065)$ & $542(0.964)$ & $-892(0.997)$ & 77 \\
\hline 13 & $\mathrm{O}^{i} \mathrm{Pr}$ & $\mathrm{Me}$ & $\begin{array}{c}248(16,500) 292(23,600) \\
484(3,210)\end{array}$ & $2.089,2.042(2.058)$ & $505(0.867)$ & $-904(0.964)$ & 77 \\
\hline 14 & O-octyl & $\mathrm{Me}$ & $\begin{array}{c}248(15,900) 291(23,600) \\
485(3,100)\end{array}$ & $2.130,2.034(2.066)$ & $545(0.929)$ & $-884(0.867)$ & 77 \\
\hline 15 & OEt & Et & $\begin{array}{c}249(18,300) 292(25,000) \\
485(3,340)\end{array}$ & $2.124,2.031(2.062)$ & $549(0.934)$ & $-874(0.975)$ & 77 \\
\hline 1 & $\mathrm{NHMe}$ & $\mathrm{Me}$ & 261314477 & $2.113,2.0292 .026(2.056)$ & 200 & -1110 & $13 a, c 8 b$ \\
\hline 30 & $\mathrm{NH}_{2}$ & $\mathrm{Me}$ & 263310477 & $\mathrm{~N} / \mathrm{A}$ & -180 & -1110 & 13 \\
\hline 31 & $\mathrm{NMe}_{2}$ & $\mathrm{Me}$ & 320450490525 & $2.0852 .0332 .024(2.047)$ & $\mathrm{N} / \mathrm{A}$ & $\mathrm{N} / \mathrm{A}$ & 8 \\
\hline 32 & $\mathrm{~N}\left(\mathrm{C}_{4} \mathrm{H}_{8}\right)$ & $\mathrm{Me}$ & 321368515566 & N/A & N/A & $-1050(1.03)$ & 39 \\
\hline 33 & $\mathrm{NPhMe}$ & $\mathrm{H}$ & 323381512567 & N/A & N/A & $-700(0.99)$ & 39 \\
\hline 34 & NHEt & $\mathrm{Me}$ & N/A & N/A & N/A & $-1120^{\mathrm{d}}$ & 94 \\
\hline 35 & NHMe & $\mathrm{H}$ & 194262324495 & N/A & N/A & -950 & 13 \\
\hline 36 & NHMe & Et & 194267316483 & N/A & 190 & -1100 & 13 \\
\hline
\end{tabular}

a) Electronic spectra measured in $\mathrm{CH}_{2} \mathrm{Cl}_{2}(\mathbf{1 0}-\mathbf{1 5})$, DMSO $(\mathbf{1}, \mathbf{3 0}, \mathbf{3 5}, \mathbf{3 6})$, or DMF (31 - 33) solution b) Powder EPR spectra collected on solids at room temperature $(\mathbf{1 0}-\mathbf{1 5}, \mathbf{1}, \mathbf{3 1}) ; g_{a v g}=1 / 3\left[g_{\|}+2 g_{\perp}\right]$. c) Electrochemical potentials versus $\mathrm{Fc}^{+} / \mathrm{Fc}$ measured versus an internal standard in $\mathrm{CH}_{2} \mathrm{Cl}_{2}$ with $0.1 \mathrm{M}^{\mathrm{n}} \mathrm{Bu}_{4} \mathrm{NBF}_{4}\left(\mathbf{1 0}\right.$ - 15) or calculated from reported potentials versus $\mathrm{Ag} / \mathrm{AgCl}$ in $\mathrm{DMSO}$ with $0.1 \mathrm{M}^{\mathrm{n}} \mathrm{Bu}_{4} \mathrm{NBF}_{4}$ $(\mathbf{1}, \mathbf{3 0}, \mathbf{3 5}, \mathbf{3 6})$ or versus $\mathrm{SCE}$ in DMF with $0.1 \mathrm{M} \mathrm{Bu}_{4} \mathrm{ClO}_{4}(\mathbf{3 2}, \mathbf{3 3})$ using the following conversions: $\mathrm{E}_{1 / 2}=-0.52$ for $\mathrm{Fc} / \mathrm{Fc} \mathrm{vs} . \mathrm{Ag} / \mathrm{AgCl}$ in DMSO; $=\mathrm{E}_{1 / 2}=-0.51$ for $\mathrm{Fc}^{+} / \mathrm{Fc}$ vs. SCE in $\mathrm{CH}_{2} \mathrm{Cl}_{2}(\mathbf{1 0}-\mathbf{1 5})$, DMSO $(\mathbf{1}, \mathbf{3 0}, \mathbf{3 5}, \mathbf{3 6})$, or DMF $(\mathbf{3 2}, \mathbf{3 3})$. d) Reported as $10 \mathrm{mV}$ more negative than $\mathbf{1}$. 


\subsubsection{Crystallographic Studies}

X-ray quality diffraction crystals of $\mathbf{1 0}-\mathbf{1 5}$ were obtained as orange needles or plates upon slow evaporation of a dichloromethane solution of the complex layered with ethanol. Crystal data and structure refinement details are listed in the Chapter 2, Tables 22 to 2-3. All complexes crystallize as discrete, square planar $\mathrm{Cu}(\mathrm{II})$ complexes with no solvent molecules in the crystal lattice. An ORTEP representation of $\mathbf{1 0}$ is shown in Figure 3-9 with selected bond distances and angles provided in Table 3-2. The structures of 11 $\mathbf{1 5}$ are highly similar to $\mathbf{1 0}$ with core structure bond distances and angles statistically indistinguishable from those in $\mathbf{1 0}$ (see Chapter 2 for $\mathrm{CCDC}$ deposit number). The following discussion of $\mathbf{1 0}$ is representative of the entire group of complexes.

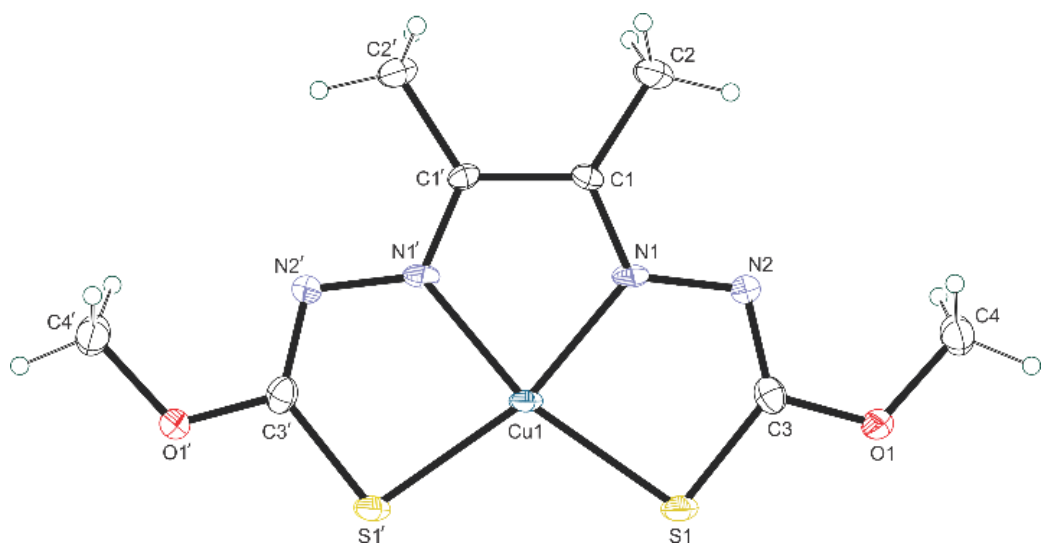

Figure 3-9. ORTEP ${ }^{95}$ view ( $50 \%$ probability) of $\mathbf{1 0}$ showing atom labeling for all non-hydrogen atoms in the asymmetric unit and symmetry generated (1-x, -y, 1/2-z) donor atoms.

The $\mathrm{Cu}$ of $\mathbf{1 0}$ occupies an $\mathrm{N}_{2} \mathrm{~S}_{2}$ square plane provided by the $N, N^{\prime}-$ bis(methylthiocarbamate)butane-2,3-diimine ligand with $\mathrm{Cu}-\mathrm{N} 1$ and $\mathrm{Cu}-\mathrm{S} 1$ bond distances of 1.958(4) and 2.2498(12) $\AA$, respectively. The S1' and N1' donors are symmetry generated as $\mathrm{Cu} 1$ sits on the special position $(0.5, \mathrm{y}, 0.25)$ in the orthorhombic space group Pbcn. A similar phenomenon occurs in 12, 14, 15, whereas in $\mathbf{1 1}$ and $\mathbf{1 3}$ the asymmetric unit contains independent atoms in the entire complex. Within the chelate ligand, bond 
distances are consistent with a conjugated $\pi$-system. One bonding MO representation can be seen in Figure 3-10. The $\mathrm{C} 3-\mathrm{S} 1, \mathrm{~N} 1-\mathrm{N} 2$, and $\mathrm{C} 1-\mathrm{Cl}^{\prime}$ bond distances of 1.741(5), 1.384(5), and 1.484(8) $\AA$, respectively, are typical of single $\mathrm{C}-\mathrm{S}, \mathrm{N}-\mathrm{N}$, and $\mathrm{C}-\mathrm{C}$ bonds. The shorter N1-C1 and N2-C3 bond distances of 1.295(5) and 1.301(6), respectively, reveal $\mathrm{C}=\mathrm{N}$ character. The sum of the four bond angles about the $\mathrm{Cu}$ is $360.0(3)^{\circ}$.

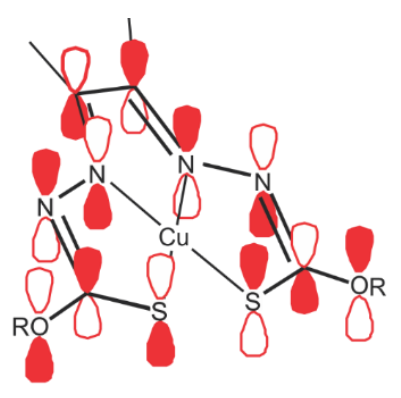

Figure 3-10. Representation of the conjugated $\pi$-network of the $N, N^{\prime}$ bis(alkylthiocarbamate)butane-2,3-diimine ligand of $\mathbf{1 0}$ highlighting localization of the $\mathrm{C}=\mathrm{N}$ bonds.

\begin{tabular}{|c|c|c|}
\hline \multicolumn{3}{|c|}{$\begin{array}{l}\text { Table 3-2. Selected bond distances }(\AA) \text { and bond } \\
\text { angles }\left({ }^{\circ}\right) \text { for } \mathbf{1 0} \text { and } \mathbf{1}^{96} \text { as representative } \\
\text { examples of } \mathrm{Cu}(\text { alkoxythiocarbamidates) and } \\
\mathrm{Cu}\left(\text { thiosemicarbazonates), respectively. }{ }^{\mathrm{a}}\right.\end{array}$} \\
\hline Bond Distance & 10 & 1 \\
\hline Cu-N1 & $1.958(4)$ & $1.9584(16)$ \\
\hline $\mathrm{Cu}-\mathrm{S} 1$ & $2.2498(12)$ & $2.2453(5)$ \\
\hline $\mathrm{N} 1-\mathrm{N} 2$ & $1.384(5)$ & $1.369(2)$ \\
\hline $\mathrm{N} 1-\mathrm{C} 1$ & $1.295(5)$ & $1.300(2)$ \\
\hline $\mathrm{N} 2-\mathrm{C} 3$ & $1.301(6)$ & $1.325(3)$ \\
\hline S1-C3 & $1.714(5)$ & $1.760(2)$ \\
\hline $\mathrm{C} 1-\mathrm{C} 1^{\prime}$ & $1.484(8)$ & $1.474(3)$ \\
\hline $\mathrm{C} 3-\mathrm{O} 1$ & $1.331(5)$ & \\
\hline $\mathrm{C} 3-\mathrm{N} 3^{\mathrm{b}}$ & & $1.338(3)$ \\
\hline \multicolumn{3}{|l|}{ Bond Angle } \\
\hline $\mathrm{N} 1-\mathrm{Cu}-\mathrm{S} 1$ & $85.00(11)$ & $85.11(5)$ \\
\hline $\mathrm{N} 1-\mathrm{Cu}-\mathrm{N} 1^{\prime}$ & $79.9(2)$ & $80.61(7)$ \\
\hline $\mathrm{S} 1-\mathrm{Cu}-\mathrm{S} 1^{\prime}$ & $110.12(7)$ & $109.21(2)$ \\
\hline
\end{tabular}

a) Atom labels for both complexes correspond to the numbering scheme of $\mathbf{1 0}$ in Figure 1. b) N3 is the nitrogen of $\mathrm{R}_{1}$ group $\mathrm{NHCH}_{3}$. 
Complex $\mathbf{1 0}$ is rigorously planar with the largest deviation from the best fit plane of all 17 non-hydrogen atoms of $\pm 0.118 \AA$ for $\mathrm{C} 3 / \mathrm{C}^{\prime}$ and a standard deviation of only $0.061 \AA$. This high degree of planarity extends throughout the whole range of complexes including the -O-octyl derivative, 14 (Figure 3-11). For 14, the best fit plane of all 31 nonhydrogen atoms of $\pm 0.364 \AA$ for $\mathrm{C} 4 / \mathrm{C} 4$ ' and a standard deviation of $0.258 \AA$. A comparison of the structural parameters of $\mathbf{1 0}$ and $\mathbf{1}$ provide a representative example of the similarities and differences of the $N, N^{\prime}$-bis(alkylthiocarbamate)butane-2,3-diimine and bisthiosemicarbazone complexes. The selected bond distances and angles in Table 3-2 reveal a highly similar structural core. The $\mathrm{Cu}-\mathrm{N} 1$ and $\mathrm{Cu}-\mathrm{S} 1$ bond lengths in $\mathbf{1 0}$ of 1.958(4) and 2.2498(12) A, respectively, are statistically equivalent with the values of 1.9584(16) and 2.2453(5) Å in 1. Within the ligand framework, the bond distances are also statistically similar. Both complexes exhibit bond distances consistent with alternating single and double bonds in an extended conjugated network as depicted in Figure 3-9 for 10. A comparison of bond angles associated with the metal-ligand core of $\mathbf{1 0}$ and $\mathbf{1}$ reveals values that are statistically equivalent or that vary less than one degree.

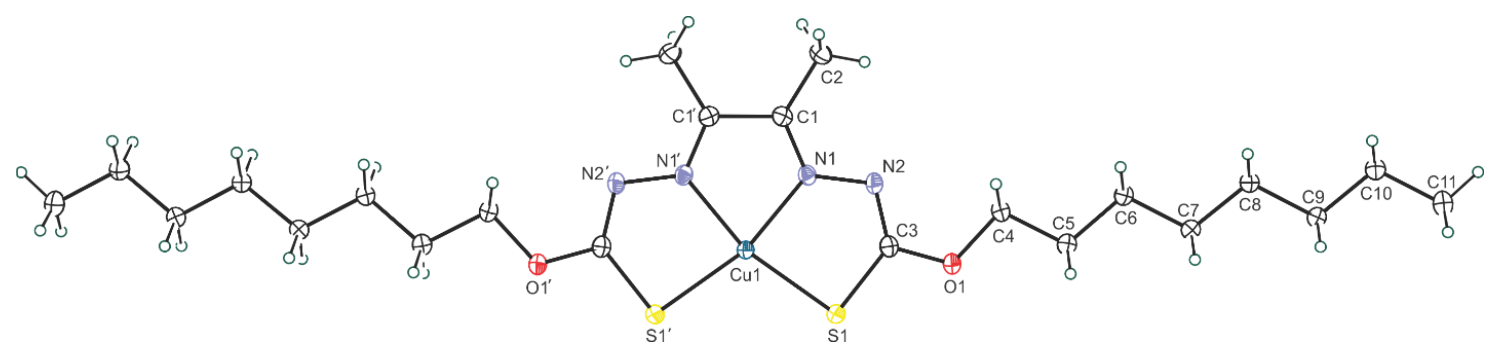

Figure 3-11. ORTEP ${ }^{95}$ view (50\% probability) of $\mathbf{1 4}$ showing atom labeling for all non-hydrogen atoms in the asymmetric unit and symmetry generated (1-x, -y, 1/2-z) donor atoms.

The degree of structural similarity makes these compounds promising for biological applications. As previously established, the structural properties of $\mathbf{1}$ and analogous 
compounds make these BTSC's suitable for diagnostic and therapeutic uses. The electrochemical properties distinguish compounds as suitable or unsuitable for therapeutic and diagnostic uses. Thus rendering the ability to maintain structural integrity with variation in the reduction potential crucial for further development of this class of compounds.

\subsection{Conclusions}

In summary, a new class of $N, N^{\prime}$-bis(alkylthiocarbamate)butane-2,3-diimine ligands and their $\mathrm{Cu}$ complexes have been reported as derivatives of bisthiosemicarbazones. Substitution of the terminal -NHR functional group with -OR allows tuning of the $\mathrm{Cu}^{\mathrm{II} / \mathrm{I}}$ and $\mathrm{Cu}^{\mathrm{III} / \mathrm{II}}$ reduction potentials with minimal impact on the physical and electronic structures of the complexes. The lack of appropriate solubility limits their biological applications and restoration of a hydrogen bond donor may increase the solubility in desired solvents. The ease of synthesis of these new ligands and the wide variety of commercially available alcohols and diones provides for a large variety of new bis-thiosemicarbazone like complexes for future development. Notably, the synthetic methodologies developed in this work will be applied in the synthesis of the hybrid complexes discussed in Chapter 5. The hydrazinecarbothioc acid $O$-alkyl esters can be condensed with a mono-keto-thiosemicarbazide to generate the hybrid complex. 


\section{CHAPTER IV}

\section{EFFECT OF STACKING INTERACTION ON THE TRANSLATION OF}

STRUCTURALLY RELATED BIS-THIOSEMICARBAZONE NI(II) HER

\section{CATALYSTS TO MODIFIED ELECTRODE SURFACES}

\subsection{Introduction}

Increasing worldwide demand for cheap energy has fueled the need to develop sustainable alternative energy sources. ${ }^{97}$ Hydrogen offers great potential as a carbon-free alternative to fossil fuels. ${ }^{98}$ One of the major challenges in this area is the development of artificial water splitting catalysts based on solid state materials and molecular based electrocatalysts that efficiently produce $\mathrm{O}_{2}$ and $\mathrm{H}_{2}$ from water utilizing either solar energy $^{99-102}$ or electrolysis. ${ }^{103-104}$ Platinum based catalysts are the gold standard catalyst for HER in aqueous acidic solutions. However, its practical widespread utility in electrocatalytic devices is limited by its scarcity and high cost. ${ }^{105-106}$ This has stimulated development of new electrocatalysts containing earth-abundant transition metals, such as $\mathrm{Fe},{ }^{107} \mathrm{Co},{ }^{108-110} \mathrm{Ni},{ }^{111-114} \mathrm{Cu},{ }^{115}$ and $\mathrm{Mo},{ }^{116-118}$ as metal phosphides, ${ }^{108,119}$ selenides,${ }^{120}$ sulfides, ${ }^{121-122}$ and small molecule complexes. ${ }^{12,34-35,74,123-125}$ Of these materials, molybdenum sulfides stand out as an economical alternative to Pt. ${ }^{126-128}$ Their high activity is thought to arise from the location and number of cis-sulfur edge sites in their layered structures. $^{128}$

There is consensus that most homogenous molecular electrocatalysts generate reactive metal-hydride intermediates during HER. ${ }^{35,129-132}$ Several of these electrocatalysts 
display low overpotential, high turnover frequencies, and Faradaic efficiencies in excess of $90 \% .{ }^{132-133}$ There is an emerging interest in the modification of electrode surfaces with small molecule HER catalysts. ${ }^{109-110,134-136}$ Most notable, Fontecave and coworkers developed multi-walled carbon nanotube $\left[\mathrm{Ni}\left(\mathrm{P}^{\mathrm{Ph}}{ }_{2} \mathrm{~N}^{\mathrm{Ph}}\right)_{2}\right]$ modified electrodes that display high catalytic activity at low overpotential. ${ }^{29}$ The Grapperhaus lab previously reported the translation of activity and mechanism of a rhenium-thiolate HER catalyst from solution to modified electrodes. ${ }^{137}$ Other examples of heterogeneous molecular catalysts for HER include $\left.\mathrm{Co}\left(\mathrm{dmgBF}_{2}\right)_{2}\left(\mathrm{CH}_{3} \mathrm{CN}\right)_{2}\right) /$ carbon black $(\mathrm{CB}) /$ Nafion coated $\mathrm{GC} 135$ and cobaloximes modified electrodes, ${ }^{135-136}$ as well as, cobalt porphyrin, ${ }^{138}$ metalphthalocyanine, ${ }^{139-142}$ and an organometallic complex incorporated in a Nafion film. ${ }^{143}$ However, these complexes either require high overpotential and/or are unstable over prolonged electrolysis reducing their practical use as HER catalysts.

Recently, the Grapperhaus lab and others have explored the use of thiosemicarbazone ligands in the design of new HER electrocatalysts. ${ }^{10,12,34-35,76}$ The thiosemicarbazone ligands are redox non-innocent and can function as a reservoir for charge with hydrogen evolution at either the metal or ligand. The HER mechanism proceeds via ligand-assisted metal-centered, ${ }^{144}$ ligand-centered, ${ }^{10,145-146}$ or metal-assisted ligand-centered reactivity depending on the identity of the metal. ${ }^{74,145,147}$ Herein, in collaboration with Alex Gupta and Dr. Gautam Gupta of the University of Louisville Engineering School, we report the synthesis, characterization, and electrocatalytic behavior of a series of structurally related bis-thiosemicarbazone Ni(II) complexes, deposited on carbon electrode surfaces. The ligands are obtained in high yields using readily available, inexpensive organic reagents according to literature methods. ${ }^{8,32}$ The resulting $\mathrm{Ni}(\mathrm{II})$ 
complexes have been characterized by NMR, FT-IR, and UV-vis spectroscopies, X-ray crystallography, and cyclic voltammetry in acetonitrile prior to deposition on glassy carbon electrodes (GCEs). Drop casting acetonitrile solutions of the Ni complexes on GCEs produce uniform coatings that were characterized by spectroscopic and microscopy methods before and after HER catalysis in aqueous acidic solutions.

\subsection{Results}

\subsubsection{Synthesis and Characterization}

The ligands $\mathrm{H}_{2} \mathrm{ATSM},{ }^{8} \mathrm{H}_{2} \mathrm{ATSDM},{ }^{32}$ and $\mathrm{H}_{2} \mathrm{ATSM}-\mathrm{F}_{6}{ }^{32}$ were prepared as previously reported. The $\mathrm{Ni}(\mathrm{II})$ complexes $\mathbf{2}, \mathbf{2 8}$, and $\mathbf{2 9}$ were synthesized using the established methodology for the preparation $2 .{ }^{8}$ The newly reported 28 and 29 were characterized by elemental analysis, cyclic voltammetry, mass spectrometry, and NMR, FT-IR, and UV-visible spectroscopies. The ${ }^{1} \mathrm{H}$ NMR spectra of $\mathbf{2 8}$ and 29, Figure 4-1 top and bottom respectively, are consistent with their proposed structures. The protons of the methyl group of the backbone, remained largely unchanged between 28 and 29 with $\delta$ values of 1.87 and 1.94 respectively. The terminal methyl protons of $\mathbf{2 8}(\delta 3.10)$ are substantially upfield of the methylene protons of $\mathbf{2 9}(\delta 4.00)$. Lastly, 29, displayed the amine proton at $\delta$ 8.28. This proton is not observed in $\mathbf{2 8}$ due to the presence of the dimethyl functionality, giving rise to the integration of the peak at $\delta 3.10$ being twice that of 1.87 .

The IR spectra of 28, Appendix, Figure A35 lacks the N-H stretching, indicative of chelation, because the metal displaces the proton. Further, it is concluded by the presence of $\mathrm{Ni}-\mathrm{S}$ and $\mathrm{Ni}-\mathrm{N}$ stretches at 900 and $783 \mathrm{~cm}^{-1}$, respectively, that the metalation was successful. The FT-IR of 29, Figure 4-2, displays the prototypical N-H stretch at $3462 \mathrm{~cm}^{-}$

${ }^{1}$ due to the presence of the pendant secondary amine. The $-\mathrm{CF}_{3}$ functionality gives rise to 
a C-F stretch at $1138 \mathrm{~cm}^{-1}$. Lastly, there is again the Ni-S and Ni-N stretches at 941 and $832 \mathrm{~cm}^{-1}$ respectively.
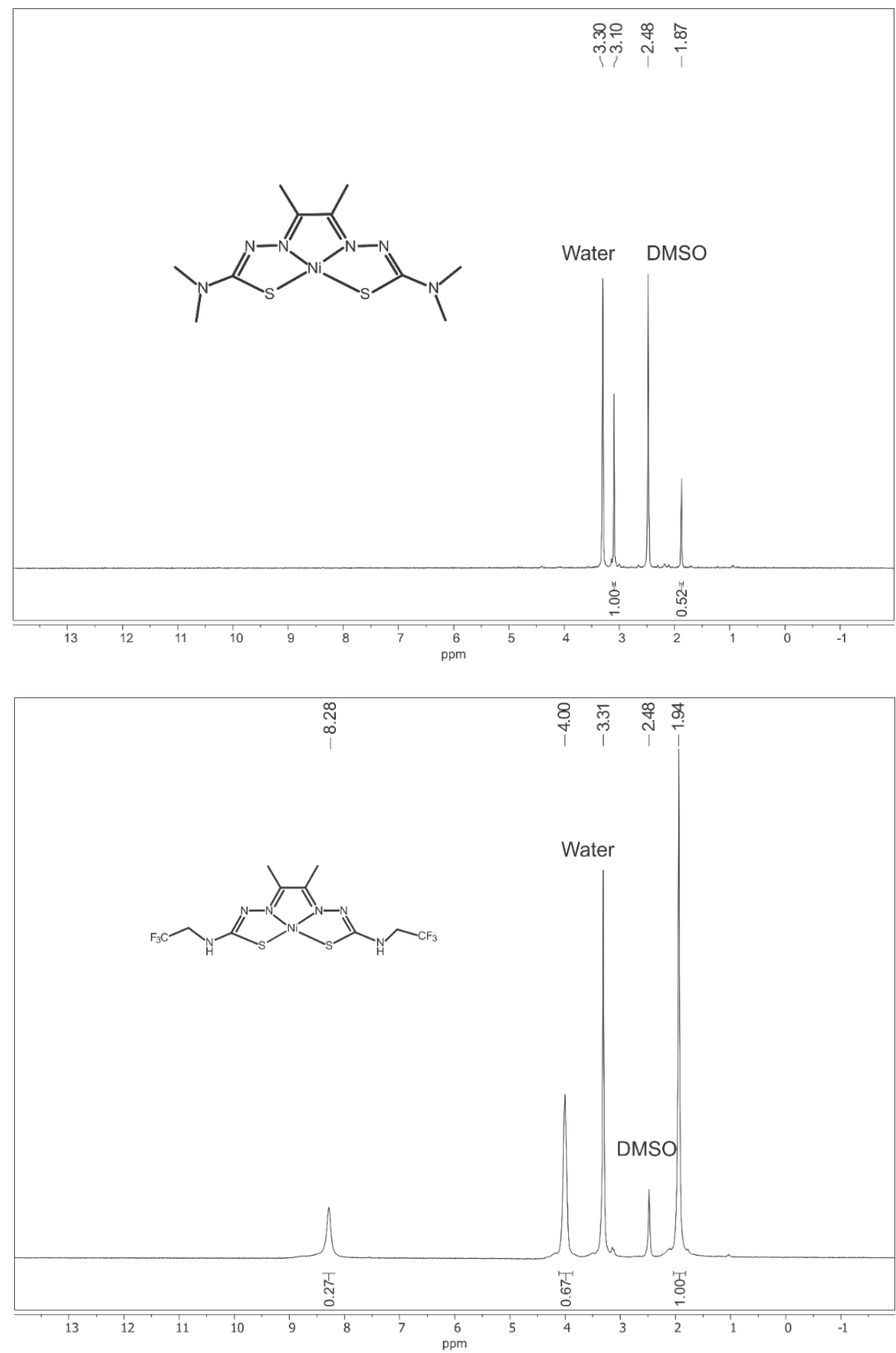

Figure 4-1. ${ }^{1} \mathrm{H}$ NMR of 28 (top) and 29 (bottom) in DMSO. 


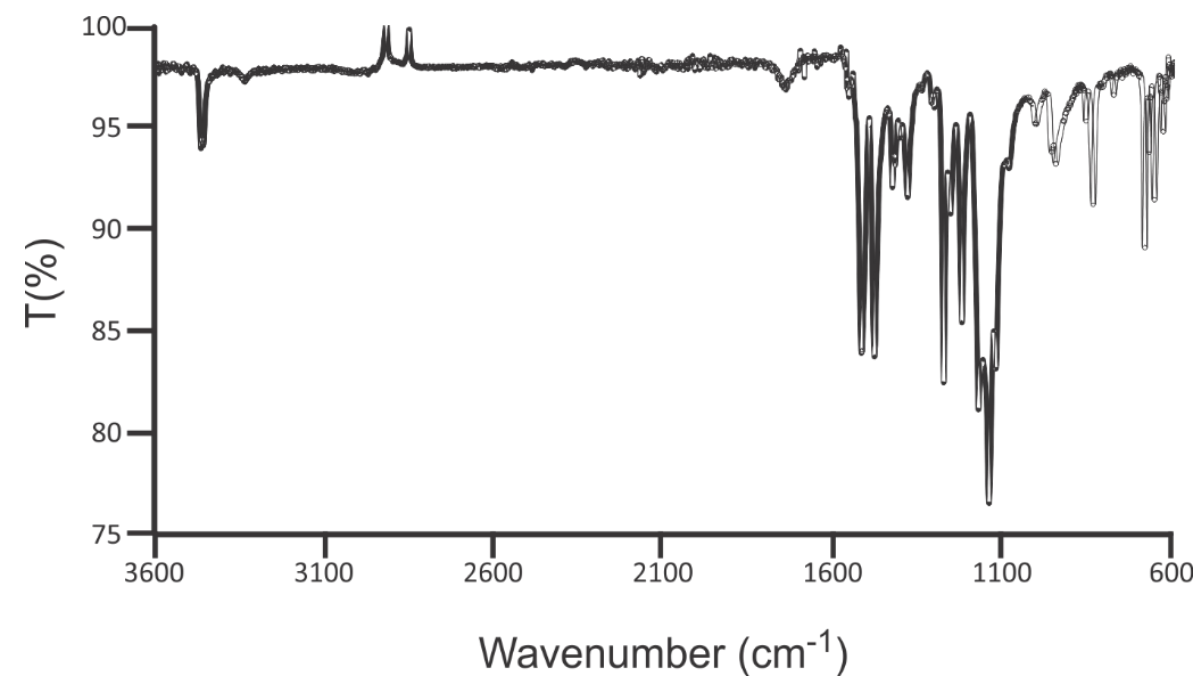

Figure 4-2. FT-IR of 29 as a powder (ATR).

All of the complexes gave green solutions in acetonitrile. The ligand-to-ligand charge transfer transitions ${ }^{8}$ in the UV-visible spectrum slightly increase in energy from $\mathbf{2 8}$ (411 and $436 \mathrm{~nm})$ to $2(398 \mathrm{~nm})$ to $29(392 \mathrm{~nm})$, Figure $4-3$. The change in the ligand to ligand charge transfer band exemplifies the effect of the functional group of the pendant amine. The energies increase from the most electron donating group, dimethyl, to the electron withdrawing group, $-\mathrm{CH}_{2} \mathrm{CF}_{3}$. Further, the ligand to ligand charge transfer band for 28 displays two $\lambda_{\max }$ values at 411 and $436 \mathrm{~nm}$. This feature appears to be absent in 2 and 29; however, the peak of $\mathbf{2}$ at $398 \mathrm{~nm}$ appears asymmetrical, indicating the possibility of a similar effect of two ligand-to-ligand charge transfer bands present. The spectrum of 29 does not readily display this feature. All complexes display similar $\pi$ to $\pi^{*}$ transitions at $256 \mathrm{~nm}$. 


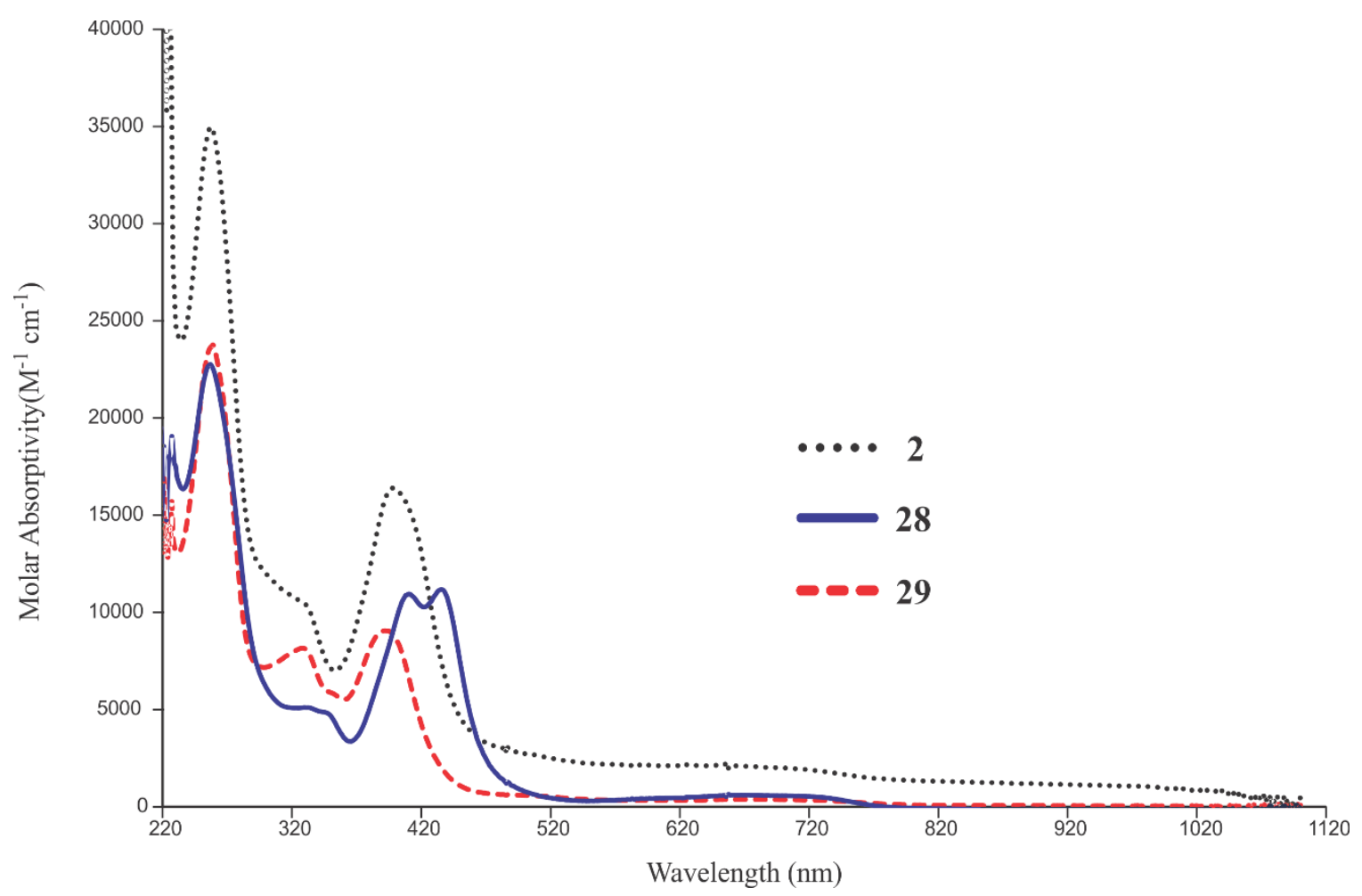

Figure 4-3. UV-visible spectra of 2 (black dots), 28 (solid blue), and 29 (dashed red) in acetonitrile.

\subsubsection{Crystallography}

The solid state structures of $\mathbf{2 8}$ and $\mathbf{2 9}$ were determined by single crystal X-ray diffraction for comparison with the known structure of $\mathbf{2} .{ }^{96}$ Crystals of $\mathbf{2 8}$ and $\mathbf{2 9}$ were obtained as orange prisms and red brown plates, respectively, via slow evaporation of an acetonitrile solution layered with ether. Crystallographic data and structure refinement details are listed in the Chapter 2, Table 2-4. The crystal lattices of $\mathbf{2}$ and $\mathbf{2 9}$ include an acetonitrile molecule, while the lattice of $\mathbf{2 8}$ is not solvated.

The ORTEP ${ }^{95}$ representations of $\mathbf{2 8}$ and 29 in Figure 4-4(top) and 4-4(bottom), respectively, show that each contains a square planar $\mathrm{Ni}(\mathrm{II})$ in an $\mathrm{N}_{2} \mathrm{~S}_{2}$ donor environment. Selected bond distances and angles for $\mathbf{2 , 2 8}$, and $\mathbf{2 9}$ are provided in Table 4-1. The Ni-N distances are statistically equivalent over the series with an average value of 1.856(5) $\AA$. 
Further, there is no significant differences in the $\mathrm{Ni}-\mathrm{S}$ bond distances, which have an average value of $2.1568(18) \AA$. The similarities in metric parameters are also observed in the bond distances within the ligand framework. For all three Ni complexes, the bond distances are consistent with a conjugated $\pi$-system of alternating single and double bonds.
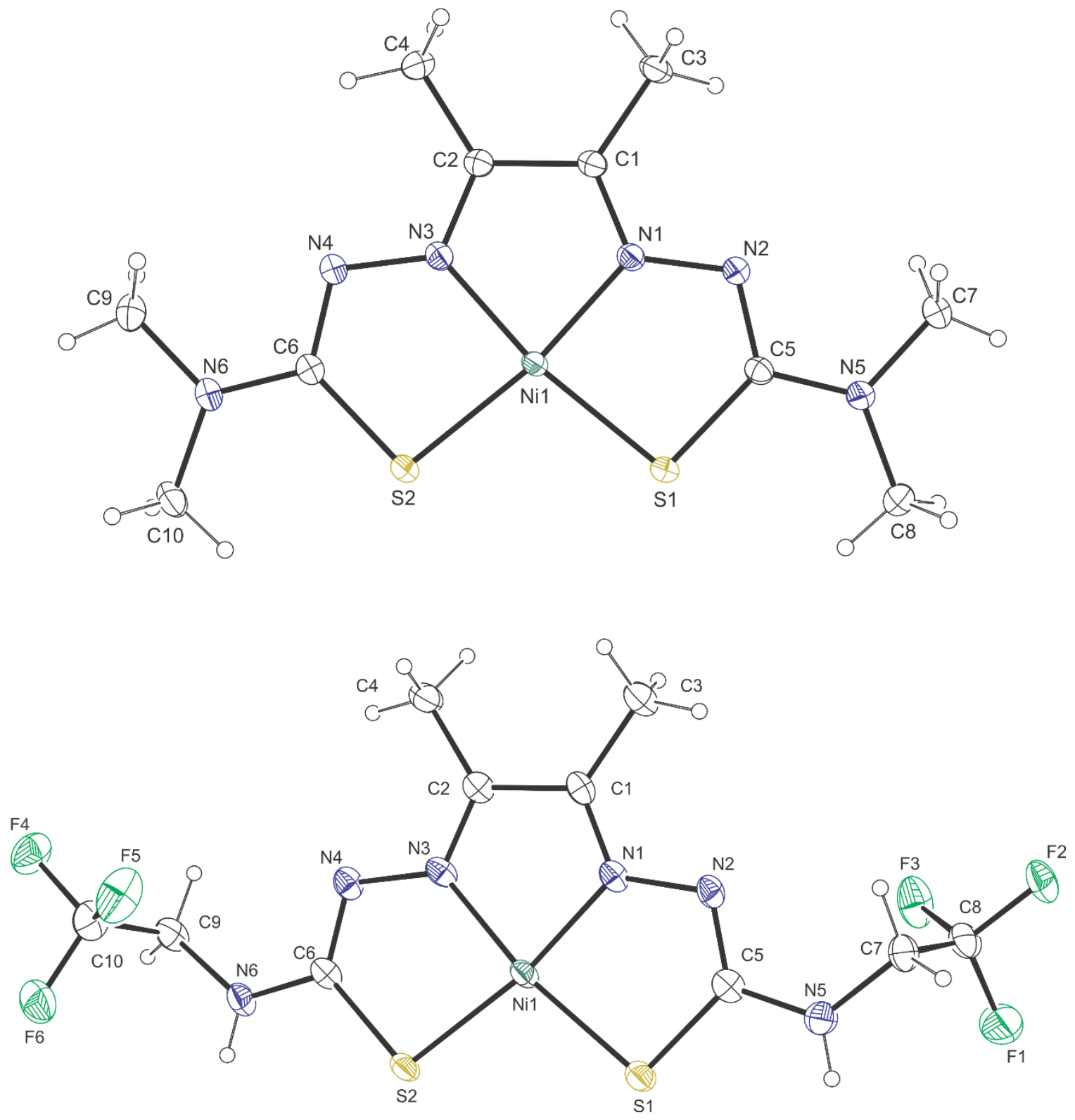

Figure 4-4. ORTEP ${ }^{95}$ view (50\% probability) of $\mathbf{2 8}$ (top) and $\mathbf{2 9}$ (bottom) showing atom labeling for all non-hydrogen atoms in the asymmetric unit. 
Table 4-1. Selected bond distances $(\AA)$ and bond angles $\left({ }^{\circ}\right)$ for $\mathbf{2},{ }^{96} \mathbf{2 8}$, and 29.

\begin{tabular}{|c|c|c|c|}
\hline Bond distance & $\mathbf{2}^{\mathrm{a}}$ & $\mathbf{2 8}$ & $\mathbf{2 9}$ \\
\hline $\mathrm{Ni}-\mathrm{N} 1$ & $1.861(2)$ & $1.8528(8)$ & $1.854(3)$ \\
\hline $\mathrm{Ni}-\mathrm{N} 3$ & $1.855(2)$ & $1.8580(8)$ & $1.863(3)$ \\
\hline $\mathrm{Ni}-\mathrm{S} 1$ & $2.1546(6)$ & $2.1617(3)$ & $2.1542(11)$ \\
\hline $\mathrm{Ni}-\mathrm{S} 2$ & $2.1598(7)$ & $2.1576(2)$ & $2.1527(10)$ \\
\hline $\mathrm{S} 1-\mathrm{C} 5$ & $1.767(3)$ & $1.7665(9)$ & $1.763(4)$ \\
\hline N2-C5 & $1.315(3)$ & $1.3222(12)$ & $1.308(5)$ \\
\hline $\mathrm{N} 1-\mathrm{N} 2$ & $1.378(3)$ & $1.3694(11)$ & $1.381(4)$ \\
\hline $\mathrm{N} 1-\mathrm{C} 1$ & $1.303(3)$ & $1.3038(11)$ & $1.310(5)$ \\
\hline $\mathrm{C} 1-\mathrm{C} 3$ & $1.489(4)$ & $1.4857(13)$ & $1.487(5)$ \\
\hline N5-C5 & $1.470(3)$ & $1.4658(13)$ & $1.474(5)$ \\
\hline N5-C7 & $1.339(3)$ & $1.3456(12)$ & $1.350(5)$ \\
\hline Bond angle & $1.449(3)$ & $1.4570(13)$ & $1.440(5)$ \\
\hline N1-Ni-S1 & $87.07(6)$ & & $87.35(10)$ \\
\hline N1-Ni-N3 & $83.59(9)$ & $87.09(3)$ & $83.65(13)$ \\
\hline S2-Ni-S1 & $101.81(3)$ & $102.019(10)$ & $101.94(4)$ \\
\hline
\end{tabular}

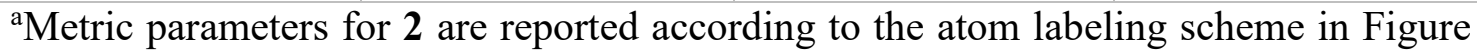
4-4, which differs from prior structural report. ${ }^{96}$

Overall, the structures of $\mathbf{2 , 2 8}$, and $\mathbf{2 9}$ are best described as square planar. Both $\mathbf{2}$ and $\mathbf{2 8}$ are rigorously planar with all non-hydrogen atoms in or near the best-fit plane. For 2, the best fit plane of all 17 non-hydrogen atoms has a standard deviation of $\pm 0.032 \AA$ with the largest deviation of $\pm 0.067 \AA$ for S2. Similarly, in 28, the standard deviation from the best fit plane of all 19 non-hydrogen atoms is $\pm 0.053 \AA$ with the largest deviation of $\pm 0.126 \AA$ for $\mathrm{C} 3$. The pendant $-\mathrm{CH}_{2} \mathrm{CF}_{3}$ groups of 29 disrupt the overall planarity of that molecule. However, with the exception of the $-\mathrm{CF}_{3}$ moieties the non-hydrogen atoms define a plane with a standard deviation of $\pm 0.033 \AA$ and a largest deviation of $\pm 0.083 \AA$ for $\mathrm{S} 2$. The $-\mathrm{CF}_{3}$ carbon atom $\mathrm{C} 8$ sits $1.46 \AA$ above the best fit plane for $\mathbf{2 9}$, whereas $\mathrm{C} 10$ lies $1.43 \AA$ below the plane. The deviation of planarity caused by the $\mathrm{CF}_{3}$ moiety can be seen in Figure 4-5. 


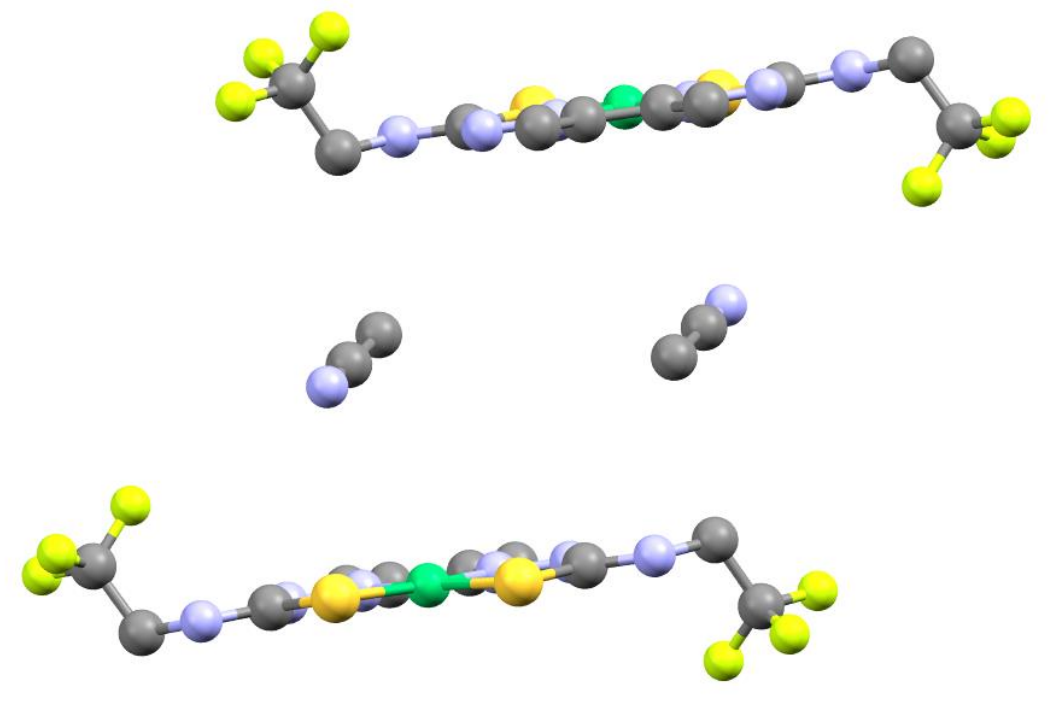

Figure 4-5. Ball and stick representation of 29 exemplifying the deviation of planarity caused by the $\mathrm{CF}_{3}$ group.

The crystal packing arrangements for the rigorously planar $\mathbf{2}$ and $\mathbf{2 8}$ display similar columns of paired complexes stacked along the $a$-axes, Figure 4-6. The bulkier $-\mathrm{CH}_{2} \mathrm{CF}_{3}$ groups in $\mathbf{2 9}$ preclude complex pairing resulting in long range stacking along the $b$-axis. For $\mathbf{2}$, the "inverse pair" of complexes containing $\mathrm{Ni}(x, y, z)$ and $\mathrm{Ni}^{\prime}(1-x, 1-y, 1-z)$ stack with an interplanar distance of $3.48 \AA$. These inverse pairs form a column of complexes generated by translation along the $a$-axis. The interplanar distance between $\mathrm{Ni}^{\prime}$ $(x, y, z)$ and $\mathrm{Ni}^{\prime \prime}(1+x, y, z)$ is $3.47 \AA$ A. Neighboring columns, with equivalent spacings, are generated by the $2_{1}$ screw axis $\left(\mathrm{Ni}^{\prime \prime \prime}\right)$ and $c$-glide $\left(\mathrm{Ni}^{\prime \prime \prime}\right)$ associated with the $\mathrm{P} 2{ }_{1} / \mathrm{c}$ space group. Complex 28, which crystallizes in the space group P-1, displays similar columns generated by translation of the inverse pair $\mathrm{Ni}(x, y, z)$ and $\mathrm{Ni}^{\prime}(1-x, 1-y, 1-z)$ along the $a$-axis with interplanar distances of 3.54 and $3.37 \AA$ for $\mathrm{Ni} / \mathrm{Ni}^{\prime}$ and $\mathrm{Ni}^{\prime} / \mathrm{Ni}^{\prime \prime}$, respectively. The crystal packing of $\mathbf{2 9}$ is unique as the bulkier $-\mathrm{CH}_{2} \mathrm{CF}_{3}$ groups prevent stacking of 
inverse pairs. As shown in Figure 4-6, $\mathrm{Ni}(x, y, z)$ and $\mathrm{Ni}^{\prime}(1-x, 1-y, 1-z)$ are contained in separate, neighboring columns directed along the $b$-axis. In contrast to $\mathbf{2}$ and $\mathbf{2 8}$, the interplanar distances for the columns of $\mathbf{2 9}$ are long, $9.40 \AA$, as each member of the column is generated by a translation in the $b$-direction.
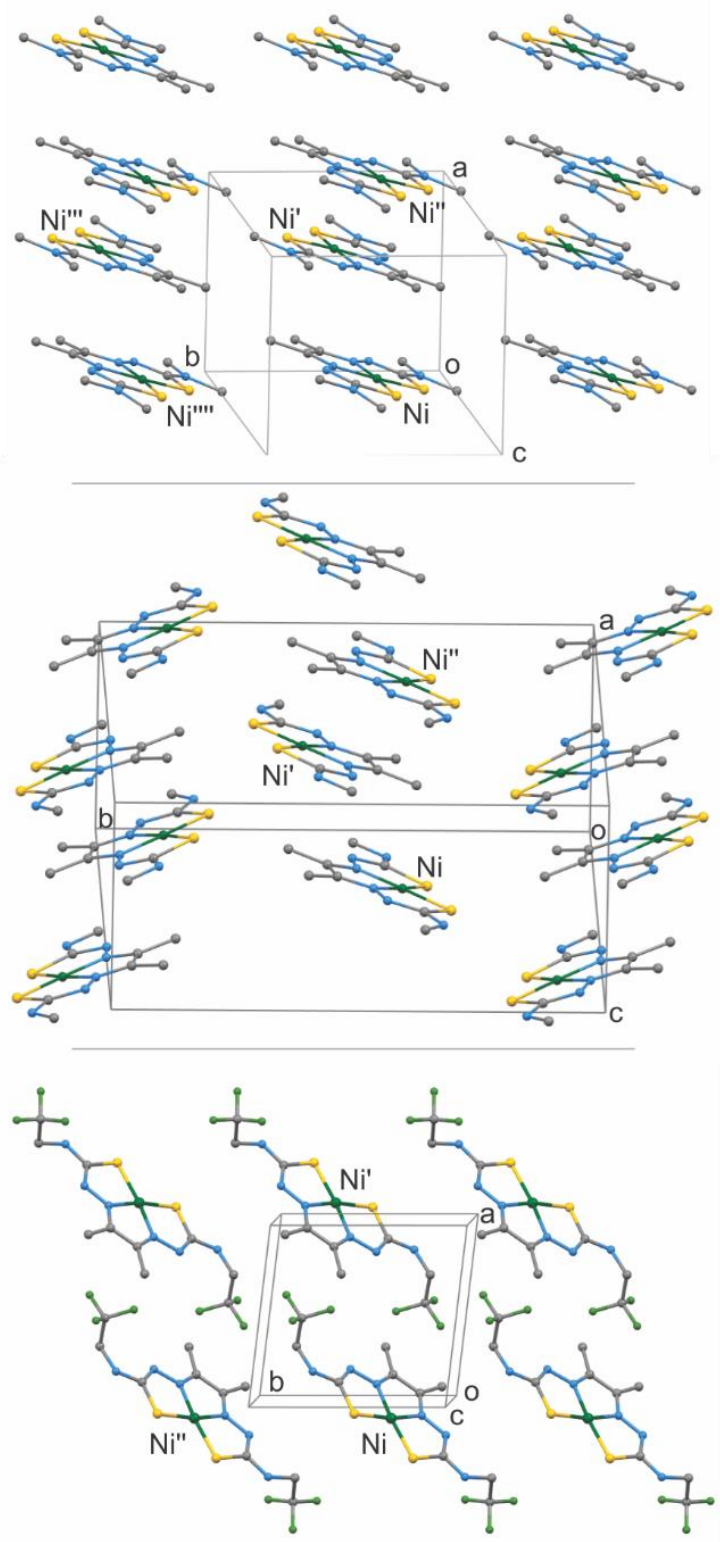

Figure 4-6. Packing diagrams for 2 (top), 28 (middle), and 29 (bottom). Individual labels are associated with the following symmetry operations: $\mathrm{Ni}, E(x, y, z)$; $\mathrm{Ni}^{\prime}, i(1-x, 1-y, 1-z), \mathrm{Ni}^{\prime \prime}$, translation along $a(1+x, y, z)$ for $\mathbf{2}$ and $\mathbf{2 8}$ or along $b(x, 1+y, z)$ for $\mathbf{2 9} ; \mathrm{Ni}^{\prime \prime \prime}, 2_{1}(1-x, 0.5+y, 1.5$ - z); $\mathrm{Ni}^{\prime \prime \prime \prime}, c$-glide $(x, 1.5-y, 0.5+z)$. 


\subsubsection{Homogeneous Electrochemical Characterization}

Cyclic voltammograms of $\mathbf{2}$ and $\mathbf{2 8}-\mathbf{2 9}$, Figure 4-7, show two reversible events at potentials more cathodic than $-1.3 \mathrm{~V}$ vs. $\mathrm{Fc}^{+} / \mathrm{Fc}$ in acetonitrile containing $0.1 \mathrm{M}$ tetrabutylammonium hexafluorophosphate (TBAHFP) as supporting electrolyte. Density Functional Theory calculations performed by Artero and co-workers ${ }^{35}$ assigned the more anodic event to a ligand-centered reduction and the more cathodic event to the $\mathrm{Ni}(\mathrm{II} / \mathrm{I})$ redox couple, Table 4-2. The ligand and metal centered $\mathrm{E}_{1 / 2}$ values for 2 occur at $-1.73 \mathrm{~V}$ and $-2.31 \mathrm{~V}$ vs. $\mathrm{Fc}^{+} / \mathrm{Fc}$, respectively. The pendant secondary amines of $\mathbf{2}$ are replaced with tertiary amines in $\mathbf{2 8}$ resulting in an $\sim 30 \mathrm{mV}$ cathodic shift in the ligand-centered reduction potential, $-1.76 \mathrm{~V}$. This is similar to corresponding $20 \mathrm{mV}$ shift observed for CuATSDM relative to CuATSM. ${ }^{13}$ The $\mathrm{Ni}(\mathrm{II} / \mathrm{I})$ event of $\mathbf{2 8}$ does not shift compared to $\mathbf{2}$. The $-\mathrm{CH}_{2} \mathrm{CF}_{3}$ functionalities of $\mathbf{2 9}$ result in a $160 \mathrm{mV}$ anodic shift of the ligand-centered event to -1.57 $\mathrm{V}$. The $\mathrm{Ni}(\mathrm{II} / \mathrm{I})$ couple of $\mathbf{2 9}$ is similarly shifted to $-2.18 \mathrm{~V}$. The magnitude of the shift is in good agreement with Que and co-workers' copper derivatives, which displayed $\sim 70 \mathrm{mV}$ anodic shift per $-\mathrm{CF}_{3}$ group when compared to CuATSM. ${ }^{32}$

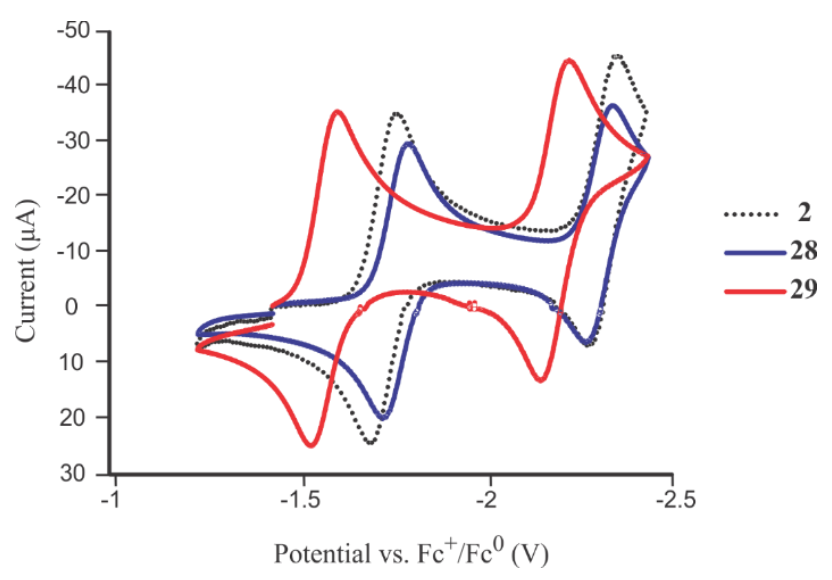

Figure 4-7. Cyclic voltammograms of 2 (black trace), 28 (blue trace), and 29 (red trace) in acetonitrile at $1 \mathrm{mM}$ concentration with $0.1 \mathrm{M}$ TBAHFP as supporting electrolyte. Scan rate $=200$ $\mathrm{mV} / \mathrm{s}$; potentials referenced versus $\mathrm{Fc}^{+} / \mathrm{Fc}$. 
Table 4-2. Reduction potentials versus $\mathrm{Fc}^{+} / \mathrm{Fc}$ and peak separation for $\mathbf{2}$ and 28- 29 in acetonitrile containing $0.1 \mathrm{M}$ TBAHFP as supporting electrolyte.

\begin{tabular}{|c|c|c|}
\hline Complex & $\mathrm{L}^{-} \mathrm{L}^{-} \mathrm{E}_{1 / 2}(\mathrm{~V}) \Delta \mathrm{E}(\mathrm{mV})$ & $\mathrm{Ni}(\mathrm{II} / \mathrm{I}) \mathrm{E}_{1 / 2}(\mathrm{~V}) \Delta \mathrm{E}(\mathrm{mV})$ \\
\hline $\mathbf{2}$ & $-1.73(78)$ & $-2.31(84)$ \\
\hline $\mathbf{2 8}$ & $-1.76(68)$ & $-2.31(73)$ \\
\hline $\mathbf{2 9}$ & $-1.57(67)$ & $-2.18(78)$ \\
\hline
\end{tabular}

\subsubsection{HER Activity of Modified Electrodes}

Modified electrodes GC-1 - GC-3 were prepared by mixing $4 \mathrm{mg}$ of 2, 28, or 29 respectively, and $12.5 \mu \mathrm{L}$ of a $10 \%$ aqueous Nafion solution with $1 \mathrm{~mL}$ acetonitrile. The resulting suspension was homogenized via ultrasonication, and $10 \mu \mathrm{L}$ of the suspension was applied dropwise to the surface of a glassy carbon electrode. The suspension was allowed to dry on the electrode with rotation at approximately 300 RPM. The freshly prepared modified electrodes required high overpotential of $\sim 700-750 \mathrm{mV}$ to reach a current density of $10 \mathrm{~mA} / \mathrm{cm}^{2}$ (the metric for comparing catalysts for water-splitting applications ${ }^{148-149}$ ) upon immersion in $0.5 \mathrm{M} \mathrm{H}_{2} \mathrm{SO}_{4}$ indicating poor initial catalytic activity.

The modified electrodes were then conditioned by reductive cycling. For GC-1 the overpotential decreases from $0.697 \mathrm{~V}$ to $0.451 \mathrm{~V}$, Figure 4-8A. Similarly, the overpotential for GC-2 decreases from $0.703 \mathrm{~V}$ to a minimum of $0.446 \mathrm{~V}$, Figure 4-8B. The large shift of $\sim 0.250 \mathrm{~V}$ represents a dramatic improvement in catalytic efficiency. Prior to conditioning, the Tafel slopes for GC-1 and GC-2 are 91 and $72 \mathrm{mV} /$ decade, respectively. Following conditioning, the Tafel slopes for GC-1 and GC-2 increase only slightly, Figures $4-8 \mathrm{D}$ and $4-8 \mathrm{E}$, suggesting the mechanism has not significantly changed. In contrast, conditioning does not improve the overpotential for GC-3, Figure 4-8C. In fact, the Tafel slope for GC-3, Figure 4-8F, increases dramatically from 53 to $167 \mathrm{mV} / \mathrm{dec}$ ade 
upon reductive cycling. This drastic change in electrode kinetics can be attributed to either a change in the active site or leaching of $\mathbf{2 9}$ into the electrolyte.
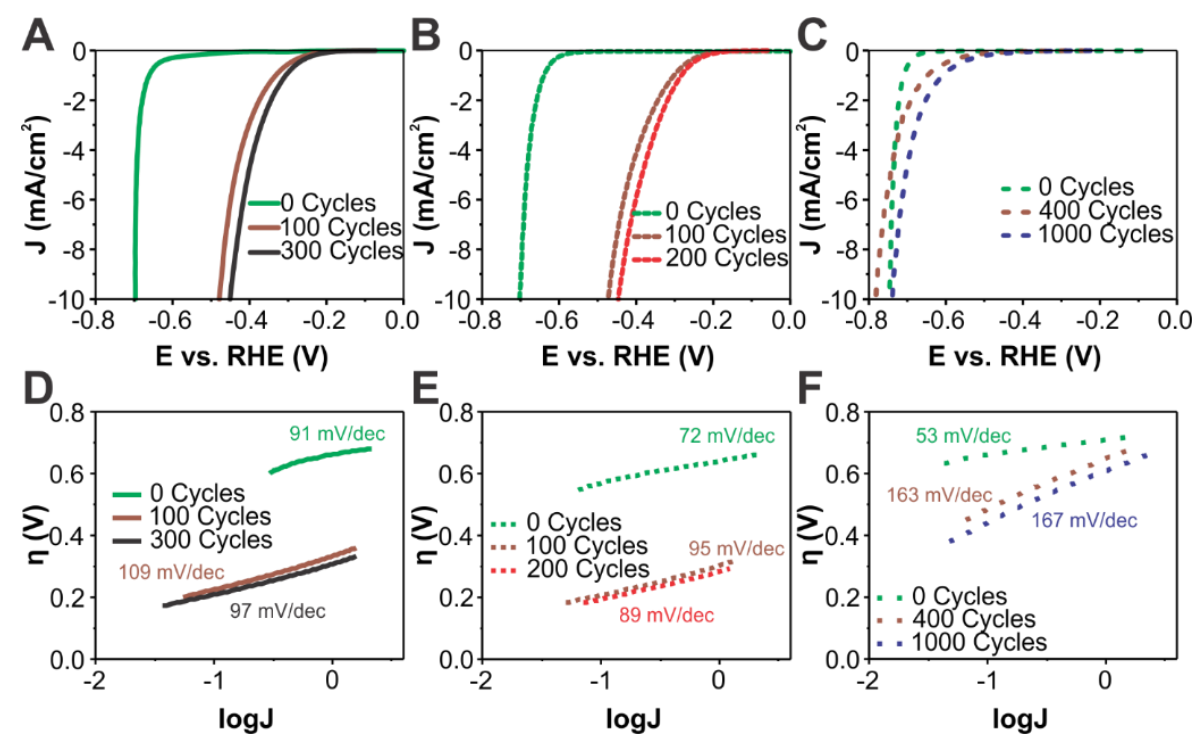

Figure 4-8. Effects of reductive cycling from 0 to $-0.8 \mathrm{~V}$ vs. RHE at $50 \mathrm{mV} / \mathrm{sec}$ on the performance of the three modified electrodes. (A) polarization curves for GC-1 exhibits peak HER performance after 300 cycles. (B) polarization curves for GC-2 exhibits peak HER performance after 200 cycles. (C) polarization curves for GC-3 exhibits peak performance after 1000 cycles. (D) Tafel slopes for GC-1 before and after 300 cycles. (E) Tafel slopes for GC-2 before and after 200 cycles. (F) Tafel slopes for GC-3 before and after 1000 cycles.

The experimentally determined Tafel plot is taken as the tangent to the onset region of the polarization curve. The onset region is traditionally defined as the region where the current is increasing while in between values of $0.5-2 \mathrm{~mA} / \mathrm{cm}^{2}$. The slope of this line is dictated by the equation slope $=\frac{-\alpha n F}{2.3 R T}$ where $R, T, n, F$, and $\alpha$ are the ideal gas constant, absolute temperature, moles of electrons transferred, Faradays constant, and transfer coefficient. For heterogeneous HER, Tafel plots provide insight into the mechanism of catalysis. From the determined Tafel slope, the rate-determining step is determined. The first step of any heterogeneous HER reaction is the Volmer step (120 mV/decade) which is the absorption of a proton to the modified electrode surface. From here, the mechanism can proceed one of two ways if the Volmer step is not rate limiting. The catalysis can 
involve the adsorbed hydrogen reacting with a free proton in solution, Heyrovsky step (40 $\mathrm{mV} /$ decade) or combination of two absorbed hydrogen atoms, Tafel step (30 mV/decade) to evolve $\mathrm{H}_{2}$, as seen in Scheme 4-1.

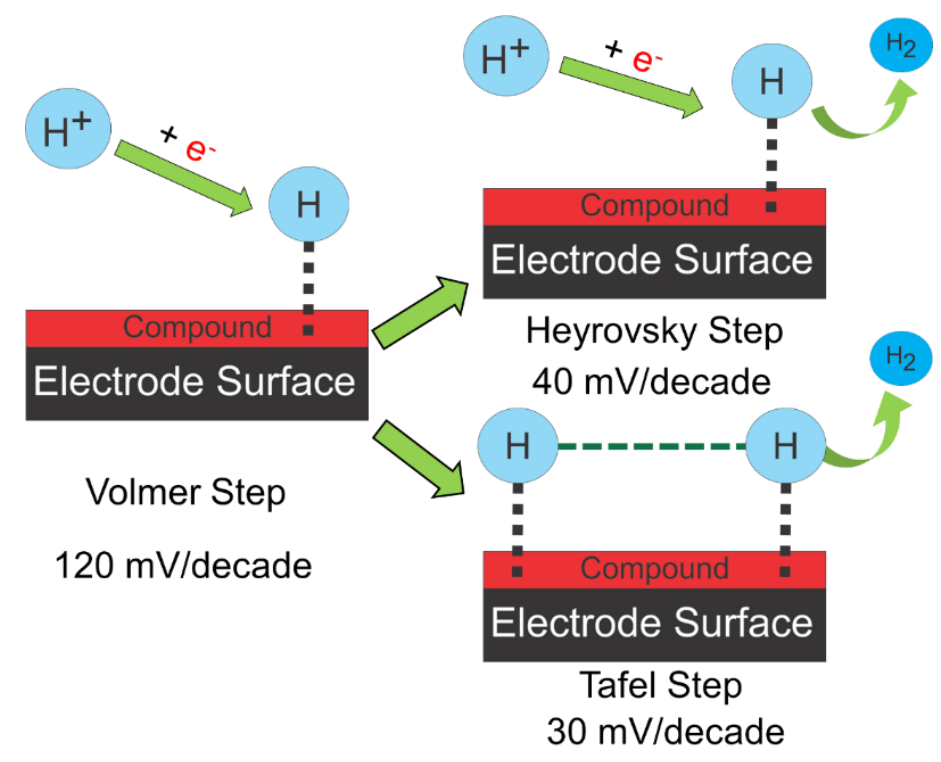

Scheme 4-1. Representations of the rate determining steps for heterogeneous HER and their corresponding Tafel slopes.

Extended reductive cycling beyond the point of conditioning was used to probe the stability of GC-1 - GC-3, Figure 4-8. No significant changes in Tafel slopes were observed over long-term cycling, suggesting no change in reaction kinetics. ${ }^{150}$ Only a minor increase in overpotential, $\sim 40 \mathrm{mV}$, was observed, consistent with a small amount of catalyst leaching into the electrolyte. For GC-1, the overpotential increases from the optimized value of $0.451 \mathrm{~V}$ after 300 cycles to $0.486 \mathrm{~V}$ after a total of 1000 cycles, Figure 4-9A. Similarly, for GC-2 the overpotential increases from $0.446 \mathrm{~V}$ after 200 cycles to $0.499 \mathrm{~V}$ after 1000 cycles, Figure 4-9B. After 1000 total cycles, the Tafel slopes for GC-1 and GC2, Figure 4-9D and 4-9E respectively, increase slightly. For GC-3, the overpotential increases from $0.740 \mathrm{~V}$ after 1000 cycles to $0.780 \mathrm{~V}$ after 1500 cycles, Figure 4-9C, with 
no significant change in Tafel slope, Figure 4-9F. The relative consistencies of the overpotential and Tafel slope over extended reductive cycling indicates this class of modified electrodes exhibits high durability for HER catalysis.

A comparison of modified electrode performance for GC-1 - GC-3 at peak electrochemical activity is summarized in Figure 4-10. The polarization curves, Figure 410A, show negligible differences in HER overpotential for GC-1 and GC-2. Both of these modified electrodes are superior to GC-3 by nearly $0.3 \mathrm{~V}$. The Tafel slopes, Figure 4-10B, for GC-1 and GC-2 of $97 \mathrm{mV} / \mathrm{dec}$ and $89 \mathrm{mV} / \mathrm{dec}$, respectively, suggest that neither proton adsorption or $\mathrm{H}_{2}$ diffusion from the active sites are rate limiting steps. ${ }^{117,151}$ The ratelimiting step is most likely the reaction of a solution proton with Ni-bound hydride to form $\mathrm{H}_{2}$ gas. The large Tafel slope of $167 \mathrm{mV} / \mathrm{dec}$ for GC-3 that implies proton adsorption is the rate-limiting step for HER catalyzed by this modified surface. ${ }^{152}$
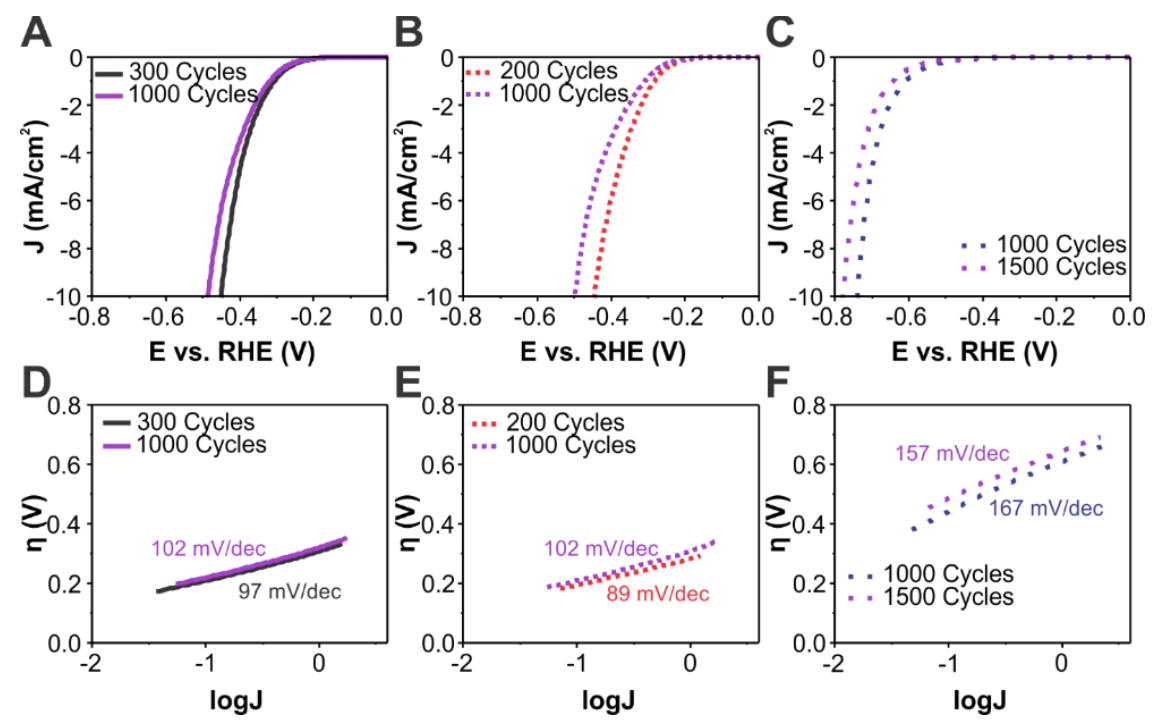

Figure 4-9. Effects of reductive cycling from 0 to $-0.8 \mathrm{~V}$ vs. RHE at $50 \mathrm{mV} / \mathrm{sec}$ on the performance of the three modified electrodes past peak activity. (A) polarization curves for GC-1, revealing that its performance diminishes following 300 cycles. (B) polarization curves for GC-2, revealing that its performance diminishes following 200 cycles. (C) polarization curves for GC-3, revealing that its performance diminishes following 1000 cycles. (D) Tafel slopes for GC-1 at 300 and 1000 cycles. (E) Tafel slopes for GC-2 at 200 and 1000 cycles. (F) Tafel slopes for GC-3 at 1000 and 1500 cycles. 


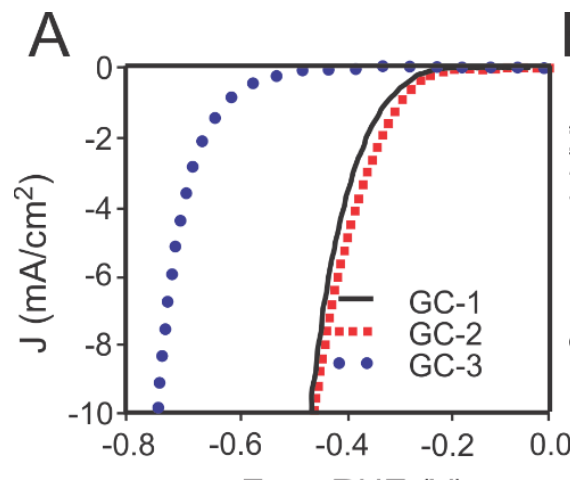

20

E vs. RHE (V)

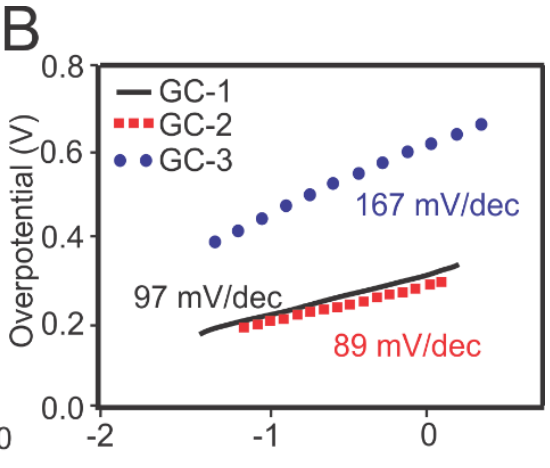

$\log \mathrm{J}$

Figure 4-10. HER performance of three nickel compounds evaluated following reductive cycling to the point of peak catalytic activity. Polarization curves (A) show the onset of hydrogen evolution and the overpotential required for each modified electrode to reach a current density of $10 \mathrm{~mA} / \mathrm{cm}^{2}$. Tafel plots (B) show the current response to increasing overpotential throughout the onset period.

This poor activity of $\mathbf{2 9}$ was perplexing since the mechanism of the NiBTSC's is proposed to be reduction first. This should then lead to 29 being the best catalyst since its reduction potentials are significantly more accessible than the other two catalysts. However, these assumptions are predicated on homogeneous catalysis. To investigate if 29 displayed better performance in homogeneous systems, 2, 28, and $\mathbf{2 9}$ were subjugated to identical homogeneous catalysis conditions, Figure 4-11.

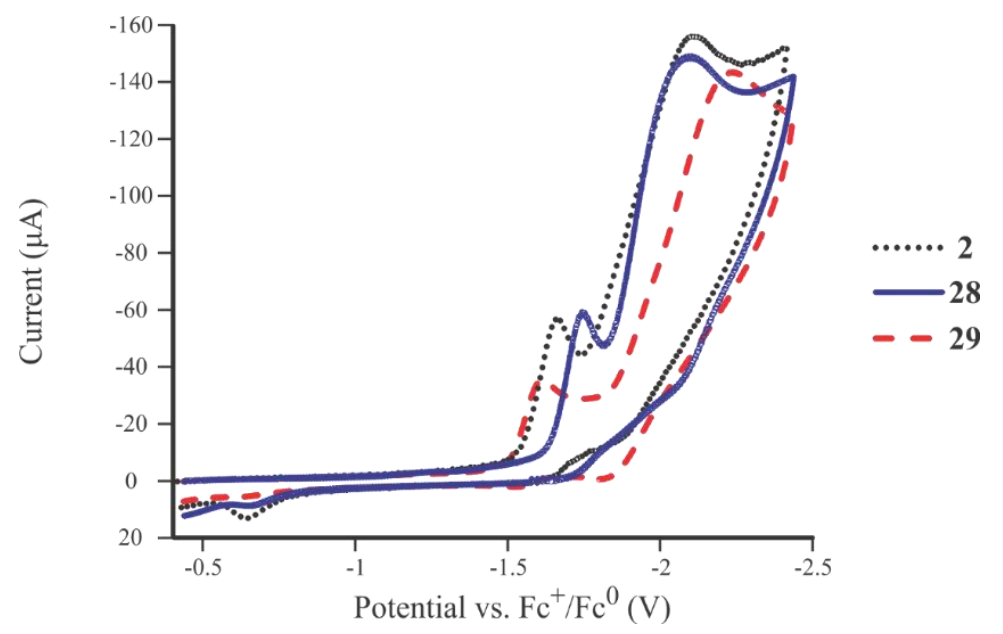

Figure 4-11. Cyclic voltammograms of 2 (black trace), 28 (blue trace) and, 29 (red trace) in acetonitrile at $1 \mathrm{mM}$ concentration, with $0.1 \mathrm{M}$ tetrabutylammonium hexafluorphospate supporting electrolyte, $16 \mu \mathrm{L}$ of acetic acid at a scan rate of $200 \mathrm{mV} / \mathrm{s}$. 
From Figure 4-11 it is observed that under identical conditions, $\mathbf{2 9}$ displays a slight cathodic shift in peak catalytic current. Interestingly, 29, has a more anodic reduction event after $-1.5 \mathrm{~V}$ compare to that of $\mathbf{2}$ and $\mathbf{2 8}$. Overall, these differences are minute and would not lead to the substantial differences in HER activity upon deposition on an electrode surface. Recently, Zhang et al. examined the effect of the electrode surface before and after reductive cycling of BTSC catalyst which revealed significant changes in morphology. ${ }^{77}$ It is believed the surface morphology changes cause the drastic differences.

\subsubsection{Surface Characterization of Modified Electrodes}

To investigate the effect of conditioning by reductive cycling, the surfaces of GC1 - GC-3 were visually characterized using SEM. Prior to conditioning, the images of GC1, Figure 4-12A, and GC-2, Figure 4-12B, show microcrystalline coatings. The crystal morphology is the same as the single crystals of $\mathbf{2}$ and $\mathbf{2 8}$ investigated by X-ray diffraction. The deposited compounds were cycled to their peak activity as established previously, to examine the surface at optimum catalysis. After conditioning, the surface of GC-1 lacks a crystalline appearance and resembles an amorphous microporous network, Figure 4-12D. A similar trend is observed for GC-2. Although the overall crystalline appearance remains intact after conditioning, Figure 4-12E, there is observable degradation of the crystalline edges and faces, Figure 4-13. In both cases, conditioning disrupts the crystal packing and reorders the catalyst on the electrode with an increase in surface area, which results in more exposed active sites. The morphology of GC-3 is very different from that of GC-1 and GC-2. Prior to conditioning, the appearance of the GC-3 surface, Figure 4-12C, is reminiscent of the thin plates of $\mathbf{2 9}$ used for single crystal X-ray diffraction studies. Following conditioning, the surface structure displays a fractured, plate-like appearance 
(Figure 4-12F) consistent with loss of complex from the surface and diminished catalyst coverage.
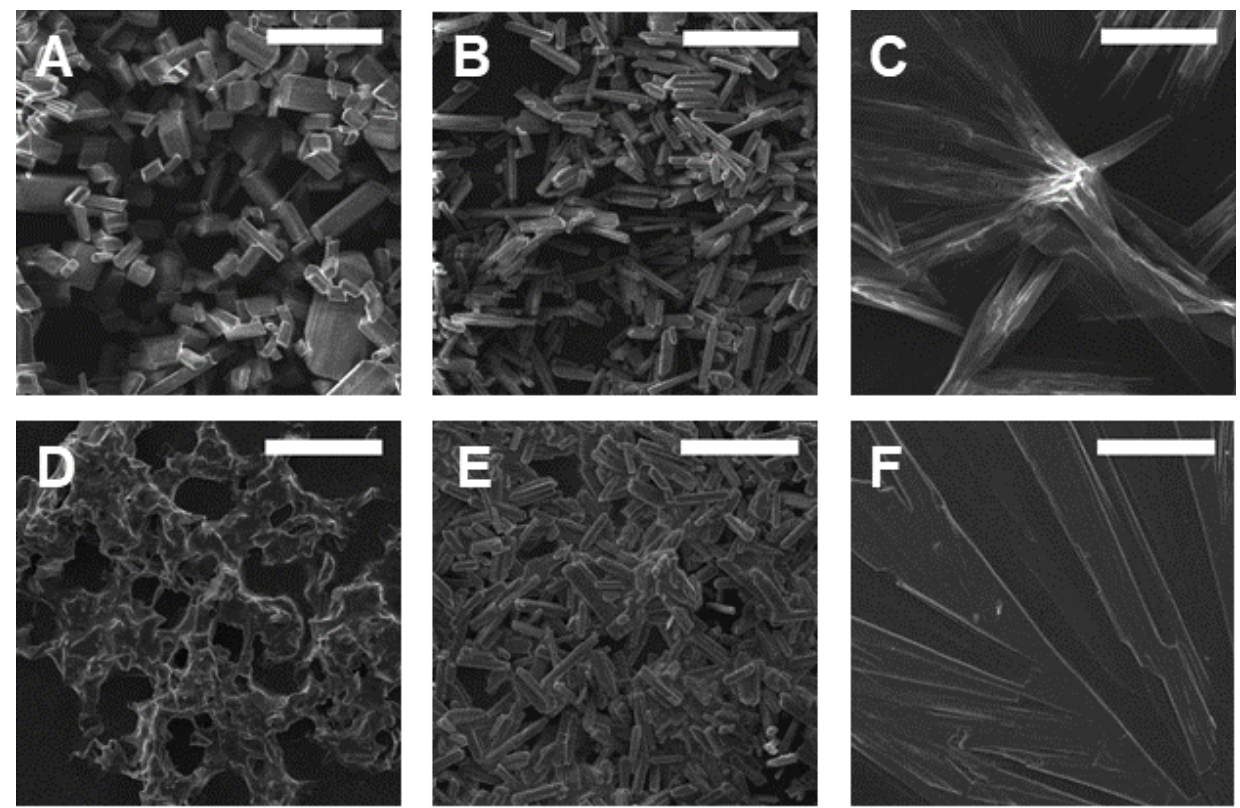

Figure 4-12. Scanning electron microscopy characterization of nickel compound films on glassy carbon electrodes before and after reductive cycling ( 0 to $-0.8 \mathrm{~V}$ vs. RHE, $50 \mathrm{mV} / \mathrm{sec}$ ) to peak activity. All scale bars correspond to $50 \mu \mathrm{m}$. (A) shows GC-1 before reductive cycling. (B) shows GC-2 before reductive cycling. (C) shows GC-3 before reductive cycling. (D) shows GC-1 after 300 cycles. (E) shows GC-2 after 200 cycles. (F) shows GC-3 after 1000 cycles.

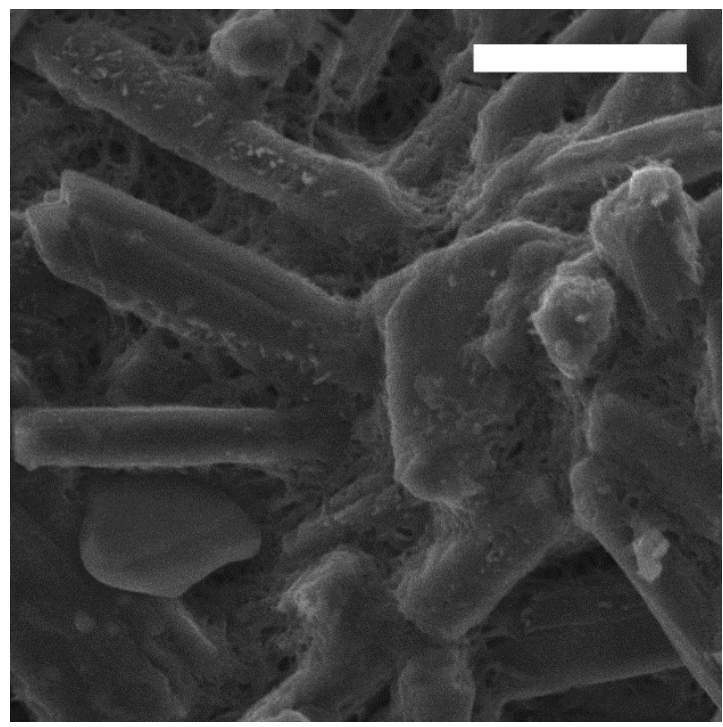

Figure 4-13. Scanning electron microscopy image of GC-2 after reductive conditioning. Image taken after reductive cycling ( 0 to $-0.8 \mathrm{~V}$ vs. RHE, $50 \mathrm{mV} / \mathrm{sec}$ ) to peak activity ( 200 cycles) highlights the fusion of crystals after cycling. Scale bar corresponds to $10 \mu \mathrm{m}$. 
The surfaces of GC-1 - GC-3 were further studied by Raman spectroscopy before and after conditioning, Figure 4-14. The Raman spectra for GC-1 and GC-2 before conditioning match those for powder samples of $\mathbf{2}$ and $\mathbf{2 8}$, respectively, confirming the successful translation of the intact complex to the GC surface. Following conditioning, the Raman spectra of GC-1 and GC-2 retain peaks associated with $\mathbf{2}$ and $\mathbf{2 8}$ clearly demonstrating the complexes remain on the surface. In contrast, for GC-3 before conditioning show intense Raman signals corresponding to the $D, G$, and $G$ ' bands of glassy carbon in addition to peaks associated with powder samples of 29. Following the reductive conditioning, the peaks associated with $\mathbf{2 9}$, such as the band at $1500 \mathrm{~cm}^{-1}$, are absent and only peaks associated with glassy carbon are observed.

This is attributed to poor initial coverage of the GC surface with substantial loss of catalyst upon reduction. The desorption of 29 is supported by the similar electrochemical activities of GC-3 following conditioning and bare GCE, Figure 4-15. Electrochemical impedance spectroscopy (EIS) was used to study the effect of conditioning on the electrical properties of the modified electrode surface. The impedance data was interpreted using an equivalent circuit model, Figure 4-16, consisting of the resistance of solution and wires $(R s)$ in series with an interfacial constant phase element (CPE) and a charge transfer resistance $(R c)$ circuit in parallel along with a Warburg element $(W)$. The CPE is a circuit element used to replace a capacitor and is generally associated with a nonhomogeneous surface and variable current density at the electrode. The impedance of the CPE is defined as:

$$
\mathrm{Z}_{\mathrm{CPE}}=1(\mathrm{j} \omega)^{-\mathrm{n}} / Q
$$


where $Q$ is the magnitude of the $\mathrm{CPE}, j$ is the imaginary unit, $\omega$ is the angular frequency, and $n$ is the CPE exponent. The Warburg element was introduced to account for diffusion resistance within crystalline domains, which are dominant prior to reductive cycling. Details are reported in the appendix, Table A1.
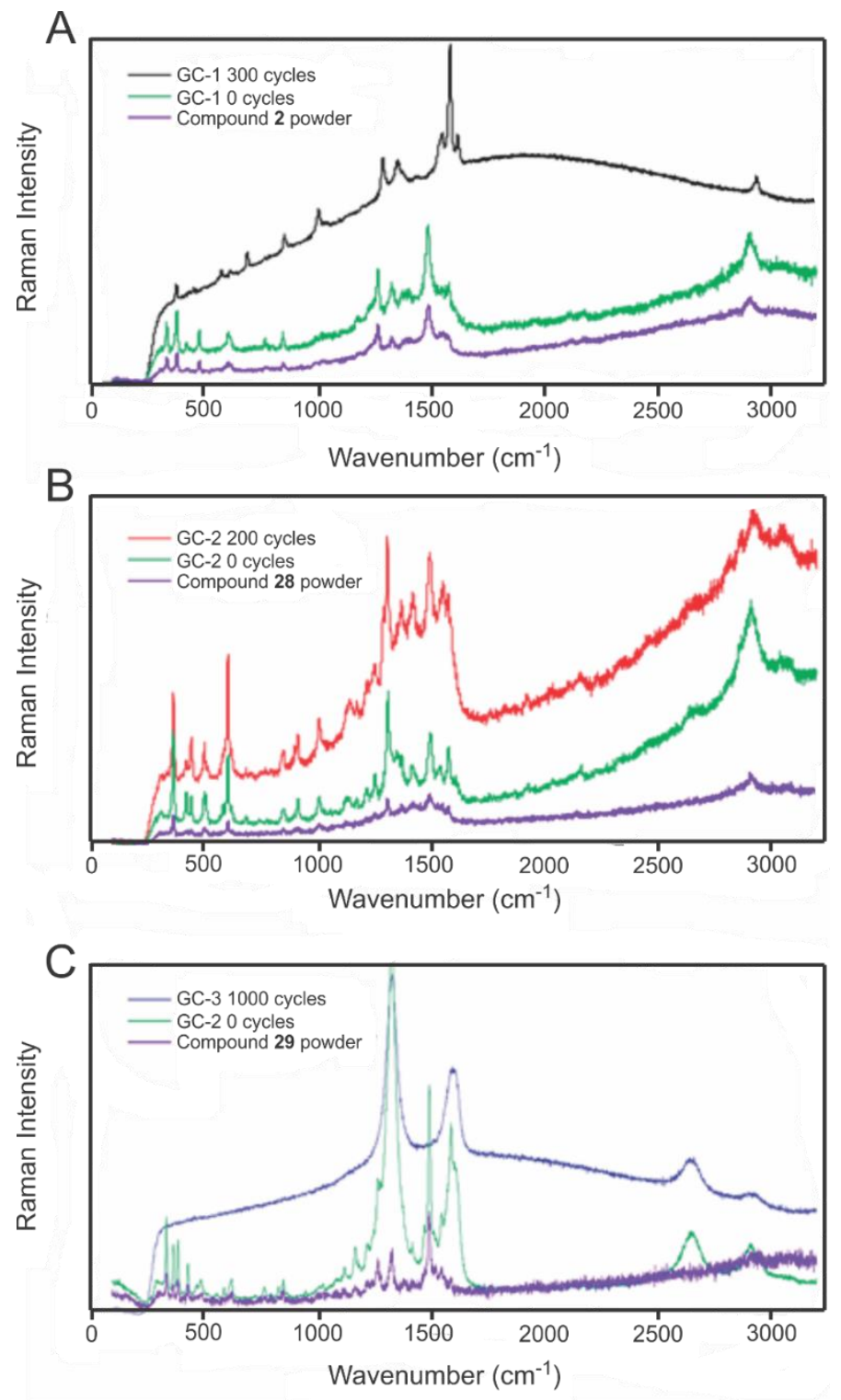

Figure 4-14. Raman spectra of 2, 28 - 29 and GC1 - GC3 before and after conditioning. Spectra were taken before and after reductive cycling ( 0 to $-0.8 \mathrm{~V}$ vs. RHE, $50 \mathrm{mV} / \mathrm{sec})$ to peak activity and nickel compound powders on glass slides. (A) shows powdered $\mathbf{2}$ (purple trace), GC-1 before cycling (green trace) and GC-1 after cycling (black trace). (B) shows powdered $\mathbf{2 8}$ (purple trace), GC-2 before cycling (green trace), and GC-2 after cycling (red trace). (C) shows powdered 29 (purple trace), GC-3 before cycling (green trace), and GC-3 after cycling (blue trace). 


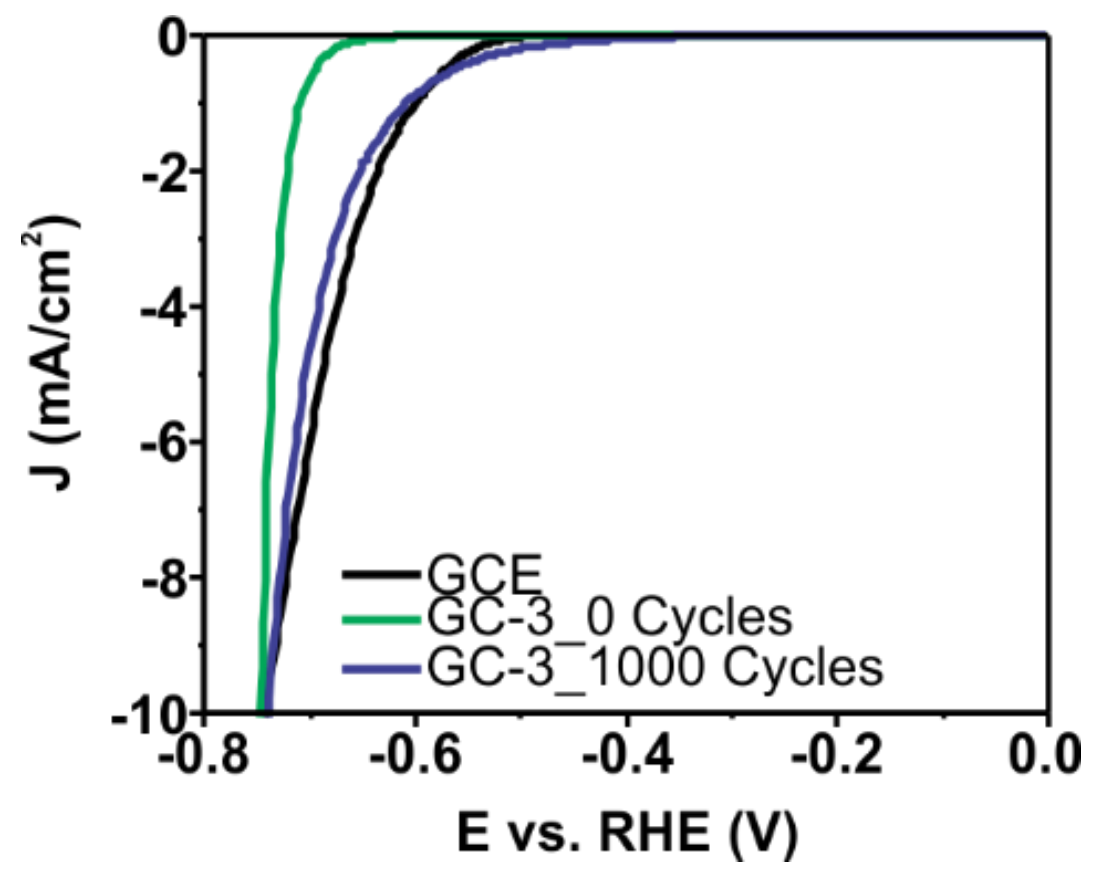

Figure 4-15. Polarization curves for bare glassy carbon (black trace) GC-3 prior to conditioning (green trace) and GC-3 after conditioning (purple trace).

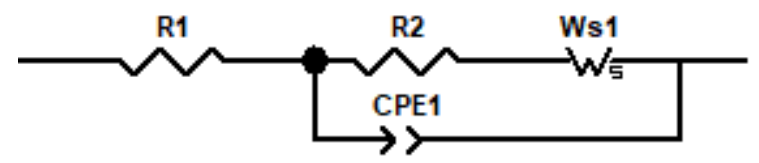

Figure 4-16. Equivalent circuit model used to fit EIS data.

Prior to conditioning, the Nyquist plots for GC-1 - GC-3 (Figure A36-A38) show

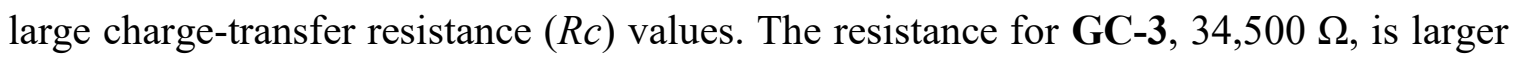
than GC-1, 25,100 $\Omega$, and GC-2, 18,800 $\Omega$. Following conditioning of 300 and 200 cycles, respectively, the charge transfer resistance for GC-1 and GC-2 drop substantially to 270 and $160 \Omega$, respectively. This dramatic decrease indicates excellent charge transfer kinetics between the film and electrode attributed to a reorganization of active sites to improve contact with the electrode and solution. In contrast, GC-3 does not exhibit significant change in charge transfer resistance upon conditioning and remains large, $34.0 \mathrm{k} \Omega$. Extended cycling to probe durability shows a slight increase in the charge transfer 
resistance for GC-1 and GC-2 to 370 and $420 \Omega$, respectively. This change is attributed to a decrease in the number of active sites due to leaching and is consistent with the small increase in overpotential.

The combined surface characterization techniques confirm morphological changes of the catalyst coatings associated with a decrease in charge transfer resistance upon exposure to reductive cycling. For GC-1 and GC-2, conditioning reorganizes the microcrystalline domains as an open network arrangement which ostensibly allows for greater solvent access and ion permeability. For GC-3, the initial catalyst film shows poor electrode coverage and reductive cycling results in significant loss of catalyst by delamination.

\subsection{Conclusions}

The immobilization of active homogeneous HER catalysts on electrode surfaces has garnered recent attention. A fundamental aspect of these studies is the ability to translate homogeneous solution phase activity to heterogeneous systems supported on an electrode. In homogeneous systems, the HER activity is largely dependent on the electronic structure of the catalyst, which dictates reduction potential and interactions with charged substrate. The physical structure can also be important as demonstrated in catalysts with pendant amines that help orient substrate for reduction. However, in heterogeneous systems

derived from small molecule catalysts the physical structure is an essential component as it relates to catalyst-electrode interactions and charge transfer between electrode, catalyst, and substrate.

The impact of small physical changes in electronically similar HER catalysts on the ability to translate activity from homogeneous to heterogeneous solution has been 
demonstrated from the evaluation of $\mathbf{2 , 2 8}$, and 29. A remarkable feature of this NiBTSC system is the good stability of the surface adhered catalysts without inclusion of covalent linkers to the surface. The non-covalent interactions between the catalyst and the electrode allow dynamic reorganization upon reductive cycling, Scheme 4-2. Complexes $\mathbf{2}$ and $\mathbf{2 8}$ are rigorously planar with the strong stacking interactions observed in single crystals, while the introduction of a $-\mathrm{CH}_{2} \mathrm{CF}_{3}$ side chain disrupts stacking interactions in the solid state of 29. The strong stacking interactions in $\mathbf{2}$ and $\mathbf{2 8}$ lead strong interaction between GC and the catalyst and films dominated by microcrystal domains. Upon reductive cycling, these domains reorganize with increased surface area and improved contact between electrode and solution. In contrast, the absence of stacking ability for $\mathbf{2 9}$ leads to poor initial electrode coverage and delamination upon reductive cycling. The results underscore the importance of physical, solid state structure for the translation of homogeneous catalysts to solid support and demonstrate how packing interactions in the crystal structure can be used to help discriminate between catalysts with similar electronic structures.

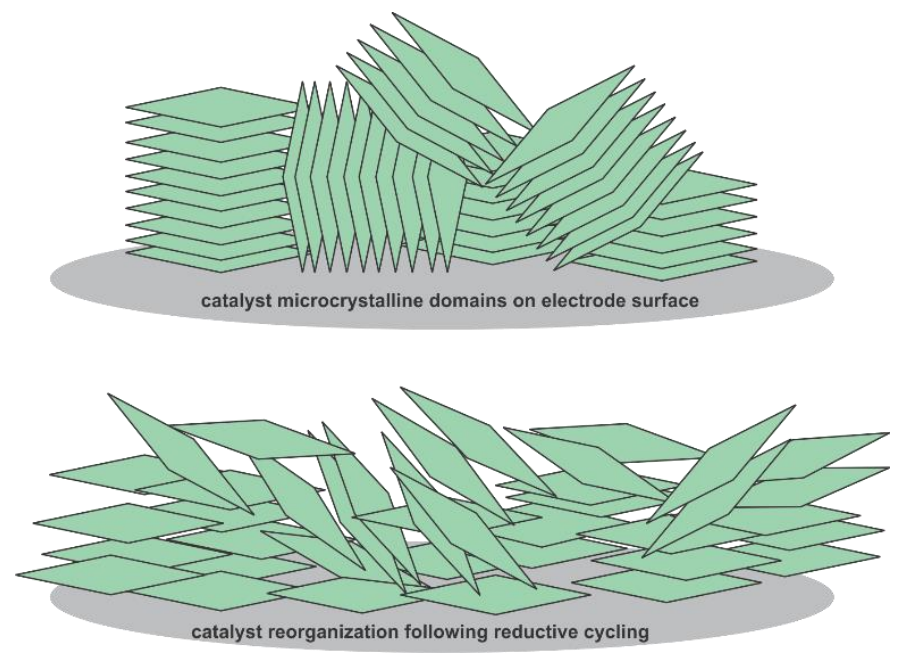

Scheme 4-2. Conditioning via reductive cycling. 


\section{CHAPTER V}

\section{SYNTHESIS, CHARACTERIZATION, AND BIOLOGICAL ACTIVITY OF NOVEL}

ASYMMETRIC BIS-THIOSEMICARBAZONE DERIVATIVES

\subsection{Introduction}

The treatment of cancer has included surgery, ${ }^{153-156}$ hormone therapy, ${ }^{157-160}$ radiation, ${ }^{161-164}$ immunotherapy, ${ }^{165-169}$ and chemotherapy. ${ }^{170-173}$ Each of these treatments come with side effects, with the most troublesome being the lack of selectivity between cancerous and healthy cells. The drive to develop a cancer therapeutic that discriminates in its apoptotic inducing behavior, has led to a new approach of targeted therapy. A prominent targeted therapy is the use of monoclonal antibodies, ${ }^{174-178}$ however, even this is not without side effects. Recently, the bis-thiosemicarbazone family of copper complexes has been garnering attention for potential biological applications with minimal side effects. ${ }^{38}$

The two most prominent bis-thiosemicarbazone complexes are $\mathbf{1}$ and CuGTSM. Both have been evaluated for in vivo applications with $\mathbf{1}$ seeing more use as a diagnostic tool for hypoxia imaging ${ }^{27-31}$ and CuGTSM as a therapeutic. ${ }^{38}$ The key difference of these two complexes lies in their backbone, which ultimately effects their $\mathrm{Cu}^{\mathrm{II} / \mathrm{I}}$ reduction potential with CuGTSM being $160 \mathrm{mV}$ more anodic. ${ }^{38}$ The ability to tune the reduction potential of these compounds allows for the exploitation of the different redox environments of cancer cells in contrast to healthy cells. Radiolabeled ${ }^{64} \mathrm{Cu}$ - and ${ }^{62} \mathrm{Cu}$ - 
ATSM have been shown to selectively remain in hypoxic tumor cells for improved imaging. The ease of modular synthesis had led to a variety of BTSC complexes being

synthesized. ${ }^{7,13,15-18,20-21,40}$ A prime example of this is the modification of the ligand framework to incorporate a small peptide sequence to enhance imaging. This synthetic methodology could be employed to enhance cell type specificity and uptake with the correct amino acid sequence. ${ }^{33}$ Further, Que and co-workers designed a novel imaging agent that eliminates the employment of radiolabeled copper and makes use of ${ }^{19} \mathrm{~F}$ MRI imaging. ${ }^{32}$

The exploitation of CuGTSMs more anodic $\mathrm{Cu}^{\mathrm{II} / \mathrm{I}}$ couple as a cancer therapeutic has led to an increase in studies on BTSCs and anti-cancer applications. ${ }^{11}$ Recently the development of cyclized derivatives, the condensation of 4-methyl-4phenylthiosemicarbazide with glyoxal, were evaluated. ${ }^{39}$ Interestingly, these compounds revealed a structure-activity relationship that suggested the mechanism of activity involving DNA strand breaking. Expanding upon the structure activity relationship revealed by the 4-methyl-4-phenylthiosemicarbazide derivatives, ${ }^{39}$ Palma et al. showed that cytotoxicity could be increased with incorporation of cyclic amines even in $\mathrm{Cu}$ BTSCs with similar $\mathrm{Cu}^{\mathrm{II} / \mathrm{I}}$ potentials to those observed for $1 .^{63}$ Further, removing the hydrogenbond donor ability of the pendant amine group has been shown to increase efficacy by preventing the system from being a substrate for MDR-1. ${ }^{65}$

All of these routes to improve the activity of $\mathbf{1}$ stem from modification of the pendant amine functional group. The recently synthesized alkoxy CuATSM derivatives 10-15 (Chapter 3) established that the structural components remained identical to the $\mathbf{1}$ while the electrochemical properties were changed. This chapter reports the synthesis, 
characterization, and electrocatalytic behavior of a series of hybrid $\mathrm{M}(\mathrm{II})$ (where $\mathrm{M}=\mathrm{Ni}$, $\mathrm{Cu}$, and $\mathrm{Zn}$ ) complexes derived from BTSCs, Scheme 5-1, for evaluation of anti-cancer properties. The ligands are obtained in high yields using readily available, inexpensive organic reagents according to literature methods. The resulting compounds have been characterized by NMR, FT-IR, and UV-vis spectroscopies, x-ray crystallography (18, 19, 21, and 22), and cyclic voltammetry.<smiles>[R]OC(=S)NNC(=S)O[R]</smiles>

\begin{tabular}{c|ll} 
Compound & $\mathrm{R}_{1}$ & \multicolumn{1}{c}{$\mathrm{R}_{2}$} \\
\hline 16 & $\mathrm{CH}_{3}$ & $\mathrm{CH}_{2} \mathrm{CH}_{3}$ \\
17 & $\mathrm{CH}_{3}$ & $\mathrm{CH}_{2} \mathrm{CH}_{2} \mathrm{CH}_{3}$ \\
18 & $\mathrm{C}_{6} \mathrm{H}_{6}$ & $\mathrm{CH}_{2} \mathrm{CH}_{3}$
\end{tabular}

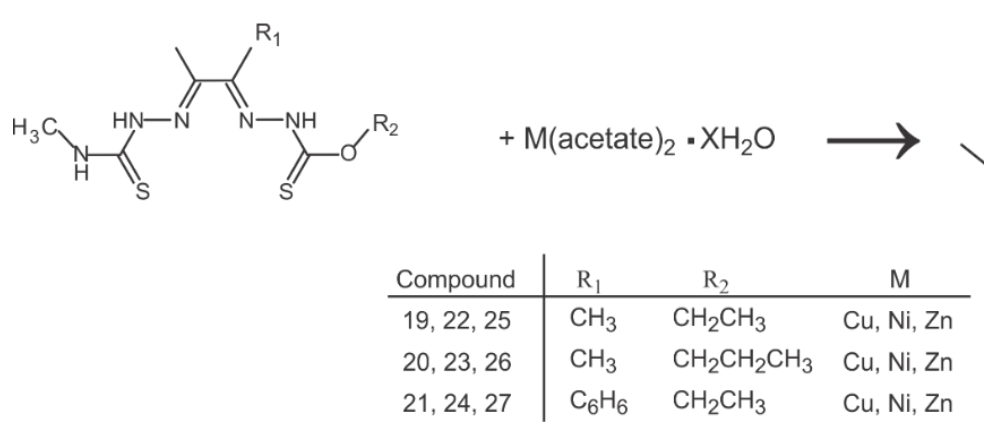

Scheme 5-1. Synthesis and numbering of ligand and metal chelates.

\subsection{Results}

\subsubsection{Synthesis and Characterization}

A series of hybrid ligands $(\mathbf{1 6}-\mathbf{1 8})$ with variation in the alkoxy alkyl chain length of ethyl (16) and propyl (17) as well as incorporation of a phenyl group in the backbone (18). The ligands were complexed with the metals $\mathrm{Cu}(\mathrm{II})(\mathbf{1 9}-\mathbf{2 1}), \mathrm{Ni}(\mathrm{II})(\mathbf{2 2}-\mathbf{2 4})$, and $\mathrm{Zn}$ (II) $(\mathbf{2 5}$ - 27). The ligands are obtained from bulk commodity chemicals. The synthetic 
routes involves those previously described in Chapter 3 for the hydrazinecarbothioc acid $O$-ethyl ester, and slight modification from previous literature reports for the mono-ketothiosemicarbazide. Briefly, dropwise addition of dilute 4-methyl-3-thiosemicarbazide to a concentrated dione solution with rapid stirring results in the mono-condensed product that is isolated via filtration. This can then be condensed with the hydrazinecarbothioc acid Oethyl ester to yield the full ligand as depicted in Scheme 5-1.

The ${ }^{1} \mathrm{H}$ NMR of 16, Figure 5-1, displays a combination of prototypical chemical shifts associated with BTSCs and the alkoxy ligands $(\mathbf{4}-\mathbf{9})$. Whereas the molecule is asymmetric, the backbone protons remain in similar magnetic environments and occur as a single peak at $2.17 \mathrm{ppm}$. The methyl protons from the pendant amine are observed as singlet at $3.00 \mathrm{ppm}$, while the methyl protons from the alkoxide moiety yield a singlet upfield at $1.28 \mathrm{ppm}$. The pendant amine proton is observed at $8.35 \mathrm{ppm}$, the iminio proton of the thiosemicarbazide side is at $10.21 \mathrm{ppm}$, and the imino proton of the alkoxide side displays the two chemical shifts of $11.31 \mathrm{ppm}$ and $11.62 \mathrm{ppm}$ (Chapter 3). The ${ }^{13} \mathrm{C}$ NMR of 16, Figure 5-2, exhibits the chemical shift for the alkoxy carbon at 66.3. The alkyl peaks of the backbone carbons, $-\mathrm{NHCH}_{3}$, and $\mathrm{CH}_{3}$ from the ethyl alkoxide are observed in the peaks at $\delta 11.8-31.7$. The $\mathrm{C}=\mathrm{N}$ is present at 179.0 and the $\mathrm{C}=\mathrm{S}$ at 152.5 and 147.9 respectively. The ${ }^{1} \mathrm{H}$ and ${ }^{13} \mathrm{C}$ NMR of $\mathbf{1 7}$ and $\mathbf{1 8}$ are similar and can be found in the Appendix (Figures A40-A43). 


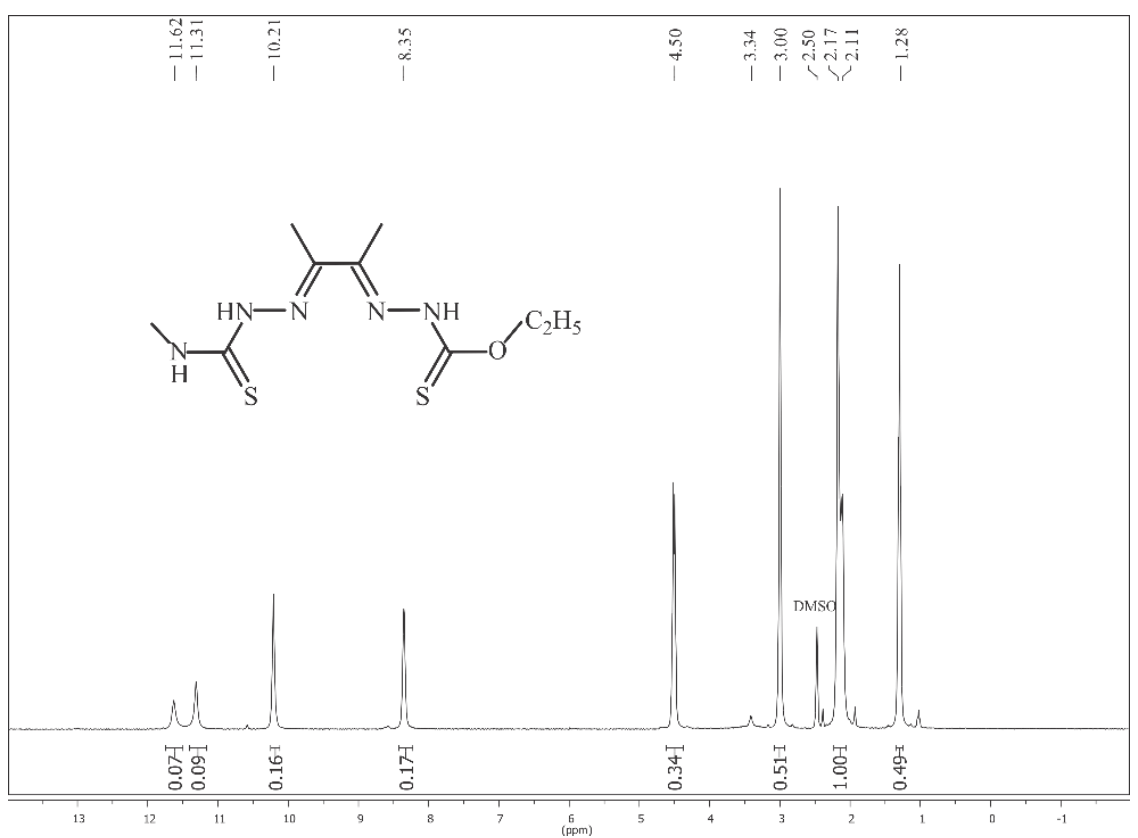

Figure 5-1. ${ }^{1} \mathrm{H}$ NMR of 16 in DMSO.

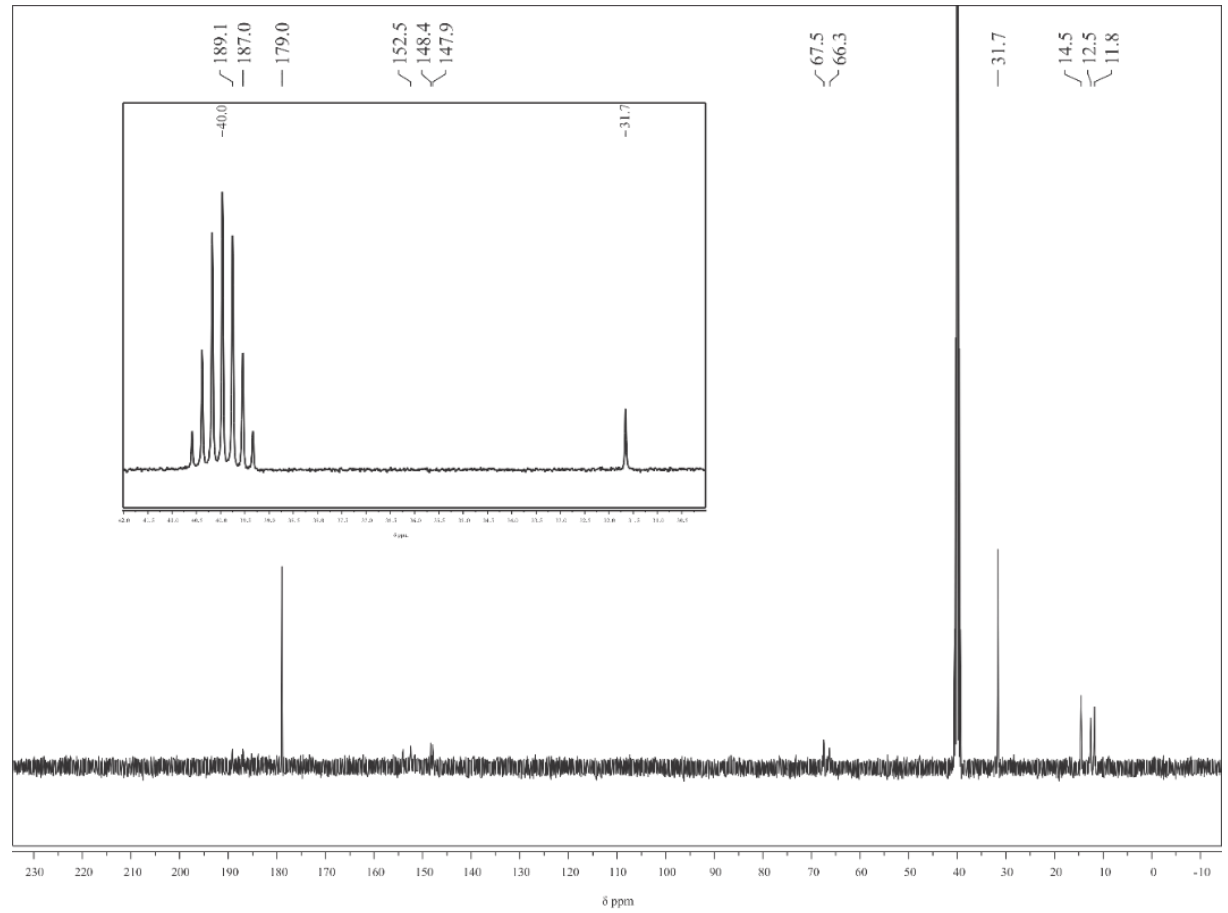

Figure 5-2. ${ }^{13} \mathrm{C}$ NMR of 16 in DMSO. Inset shows DMSO peak.

The FT-IR of 16 (Figure 5-3) is representative of the class of ligands with the remaining found in Appendix (Figures A44-A45). Notably, 16 displays a combination of 
hallmark stretches of both BTSCs, two N-H stretches at 3382 and $3253 \mathrm{~cm}^{-1}$, and the previously synthesized alkoxy ligands, $\mathrm{C}-\mathrm{O}$ at $1130 \mathrm{~cm}^{-1}$. Upon metalation with copper (19), Figure 5-3 B, the $\mathrm{N}-\mathrm{H}$ stretch present at $3382 \mathrm{~cm}^{-1}$ is absent consistent with deprotonation of the imino hydrogen. However, an $\mathrm{N}-\mathrm{H}$ stretch is observed at $3305 \mathrm{~cm}^{-1}$ due to the presence of the pendant amine. The $\mathrm{C}-\mathrm{O}$ stretch remains largely unaffected at $1132 \mathrm{~cm}^{-1}$. Further, the addition of stretches at 846 and $782 \mathrm{~cm}^{-1}$ are attributed to $\mathrm{C}-\mathrm{S}$ and $\mathrm{Cu}-\mathrm{N}$ bonds respectively. Similar stretches, such as the appearance of $\mathrm{Ni}-\mathrm{S} / \mathrm{Ni}-\mathrm{N}$ and $\mathrm{Zn}-$ $\mathrm{S} / \mathrm{Zn}-\mathrm{N}$ are observed in the remaining FT-IR spectra and can be found in the Appendix (Figures A46 - A51).
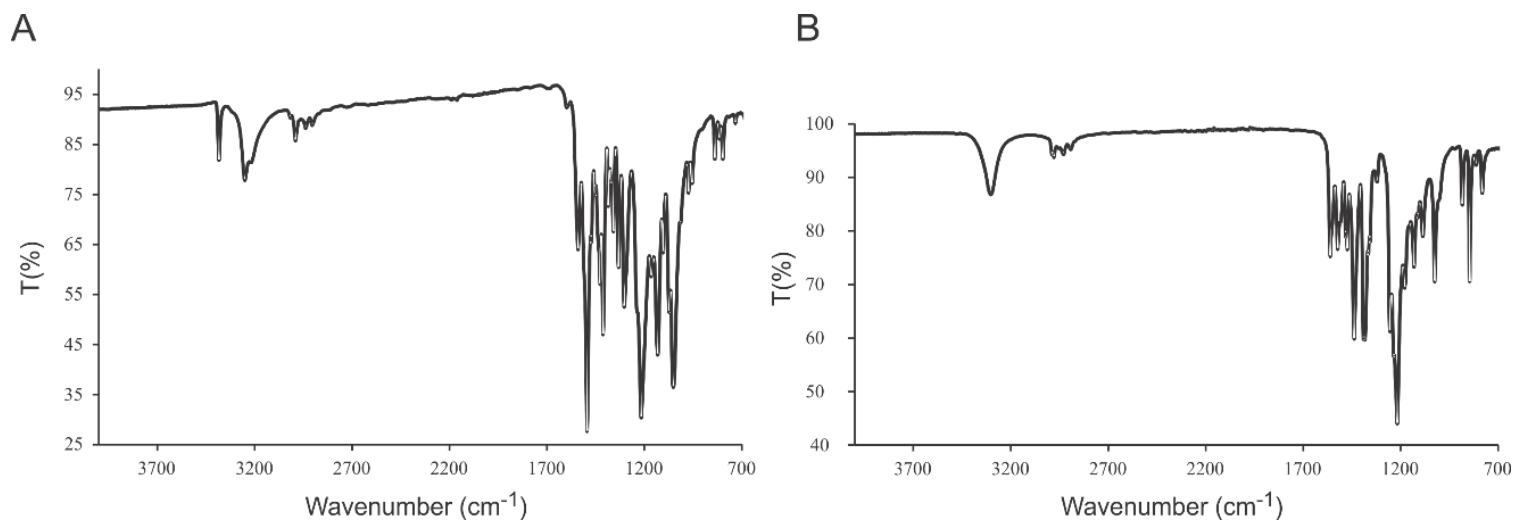

Figure 5-3. FT-IR of 16 (A) and 19 (B) as powders.

When 16 is chelated with zinc (25) the ${ }^{1} \mathrm{H}$ NMR (Figure 5-4) displays full deprotonation of the imino hydrogens with the absence of a peaks at 10.21 and $11.31 / 11.62$ ppm. This trend is consistent with the remaining zinc complexes (Figures A52-56). The nickel derivatives display a similar ${ }^{1} \mathrm{H}$ NMR pattern, however in all nickel complexes a peak at 8.55 is present (Figures A57-A59). This is not attributed to the imino hydrogens as those are over 2 ppm downfield, and in the FT-IR (Figure 5-5) of 22 there is only one N$\mathrm{H}$ stretch. This peak is attributed to a small amount of residual acetic acid. 


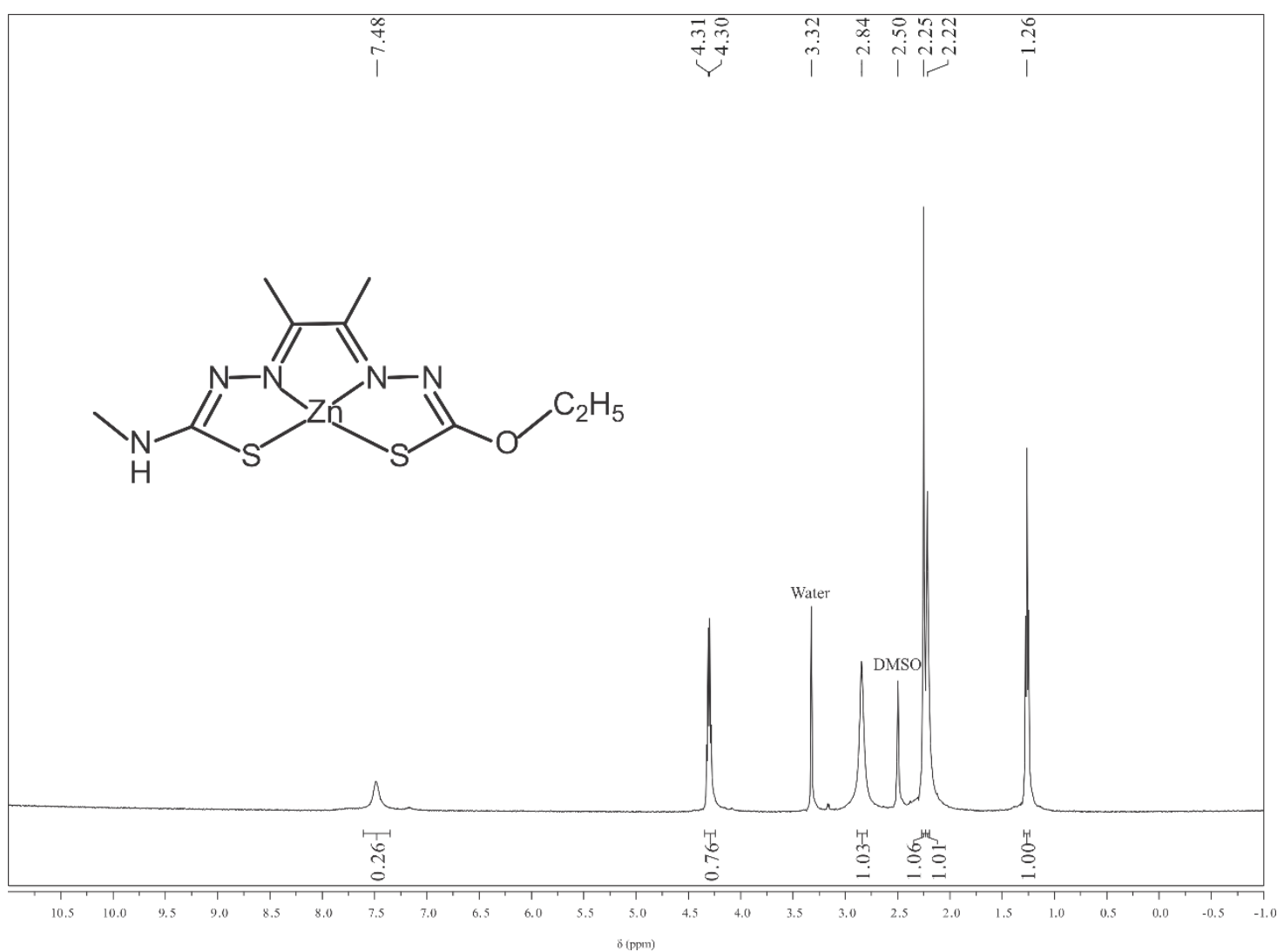

Figure 5-4. ${ }^{1} \mathrm{H}$ NMR of 25 in DMSO.

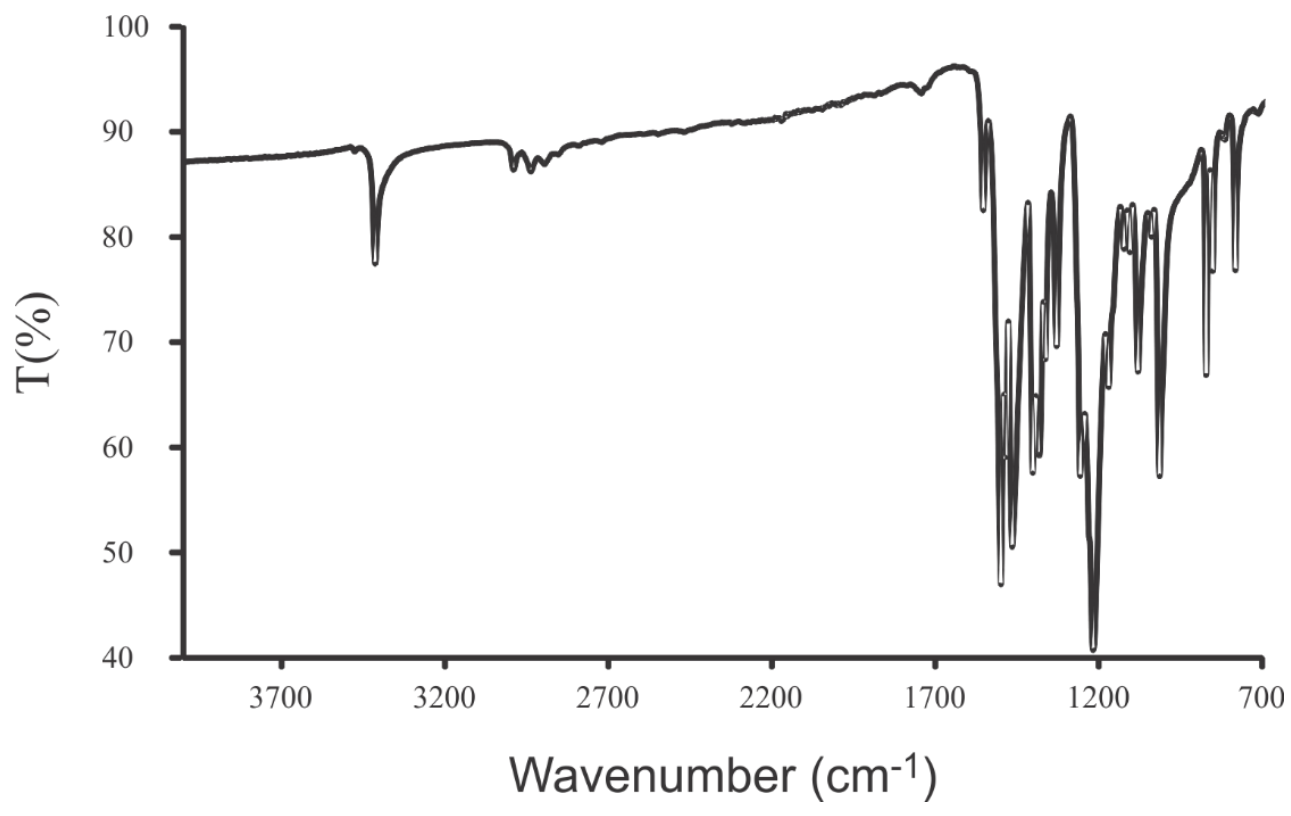

Figure 5-5. FT-IR of 22 as a powder. 
The electronic spectra of $\mathbf{1 9} \mathbf{- 2 7}$ were collected in acetonitrile, and all display two ligand to ligand charge transfer bands and one metal to ligand charge transfer band. The electronic spectra of the $\mathrm{Cu}$ complexes $(\mathbf{1 9}-\mathbf{2 1})$ are displayed in Figure 5-6. Compounds 19 and 20 contain ligand to metal charge transfer bands at 477 and $473 \mathrm{~nm}$ respectively. The charge transfer band in 21 is redshifted by $346 \mathrm{~cm}^{-1}(8 \mathrm{~nm})$ to $485 \mathrm{~nm}$ relative to 19 . This redshift is observed within the higher energy ligand to ligand charge transfer values. The recorded ligand to ligand charge transfer band for $\mathbf{1 9}$ and $\mathbf{2 0}$ are observed at 248 and $249 \mathrm{~nm}$. For 21 this value is red shifted by $2,300 \mathrm{~cm}^{-1}$ relative to 19 , to $263 \mathrm{~nm}$. Interestingly, the lower energy band remains consistent in all complexes at a value of 309 $\mathrm{nm}$. The remaining electronic spectra of $\mathrm{Ni}(\mathbf{2 2}-\mathbf{2 4})$ and $\mathrm{Zn}(\mathbf{2 5}-\mathbf{2 7})$ can be found in the Appendix (Figures A60-A61). In both of these systems the redshift is observed for the phenyl derivative, $\mathbf{2 4}$ for the $\mathrm{Ni}$ and $\mathbf{2 7}$ for the $\mathrm{Zn}$. In the Ni system, however, the high energy ligand to ligand charge transfer band remains similar with all occurring around 255 $\mathrm{nm}$. The lower energy band however has values of 329 and $330 \mathrm{~nm}$ for $\mathbf{2 2}$ and $\mathbf{2 3}$ respectively, whereas 24 appears at $342 \mathrm{~nm}$, a redshift of $1156 \mathrm{~cm}^{-1}$. Lastly, in the $\mathrm{Zn}$ complexes, all charge transfer bands of $\mathbf{2 7}$ are redshifted relative to $\mathbf{2 5}$ and $\mathbf{2 6}$. The ligand to ligand charge transfer bands are observed at 243 and $299 \mathrm{~nm}$ for 25 and the metal to ligand charge transfer occurring at $422 \mathrm{~nm}$. However, in 27 the corresponding transfer bands occur at 269, 326, and $430 \mathrm{~nm}$, redshift values of $3,978 \mathrm{~cm}^{-1}, 2,770 \mathrm{~cm}^{-1}$, and 441 $\mathrm{cm}^{-1}$. 


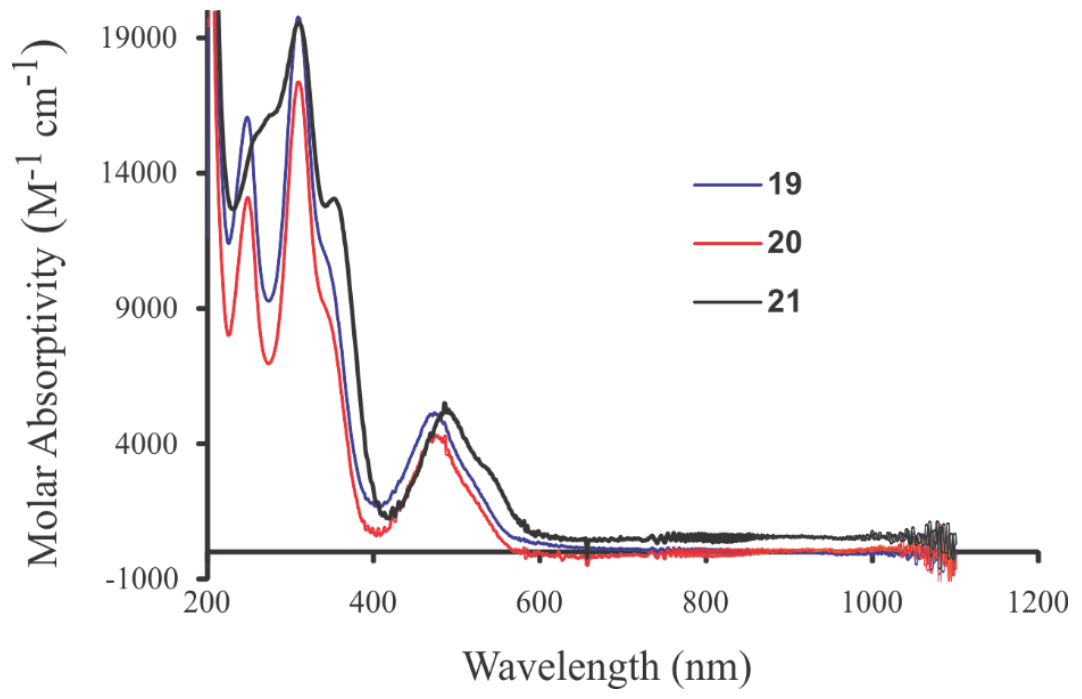

Figure 5-6. UV-visible spectra of 19 (blue trace), 20 (red trace), and 21 (black trace) recorded in acetonitrile.

\subsubsection{HER Catalysis Evaluation}

We had previously established that $\mathbf{1}, \mathbf{3}$, and its ligand, ${ }^{10,34}$ are viable for proton reduction catalysts. As described in Chapter 3, substitution of the -NHMe groups of 1 with the-OMe of $\mathbf{1 0}$ results in a large anodic shift in the reduction potential. Such a shift could potentially lower the overpotential of these types of HER catalysts. To this end $\mathbf{1 0}$ was evaluated as a possible HER catalyst, but ultimately failed. The absence of HER activity was attributed to the first step of the mechanism of HER evolution of CuATSM, protonation. A ligand framework with electron withdrawing groups (EWG) can lower reduction potentials. However, the employment of these EWGs leads to a loss of basicity in the ligand framework. To rectify this situation, a hybrid ligand was developed in which one pendant group was -NHMe and one was-OEt. The complex was further modified by incorporation of a phenyl group in the backbone, 21. Under catalytic conditions 21 (blue trace), was found to produce hydrogen unlike $\mathbf{1 0}$ (red trace), Figure 5-7. Ultimately though 21 did not produce $\mathrm{H}_{2}$ at a reasonable overpotential. To investigate this, spectrophotometric 
titrations were employed. CuATSM was used as the standard since at this point it was the best performing CuBTSC HER catalyst.

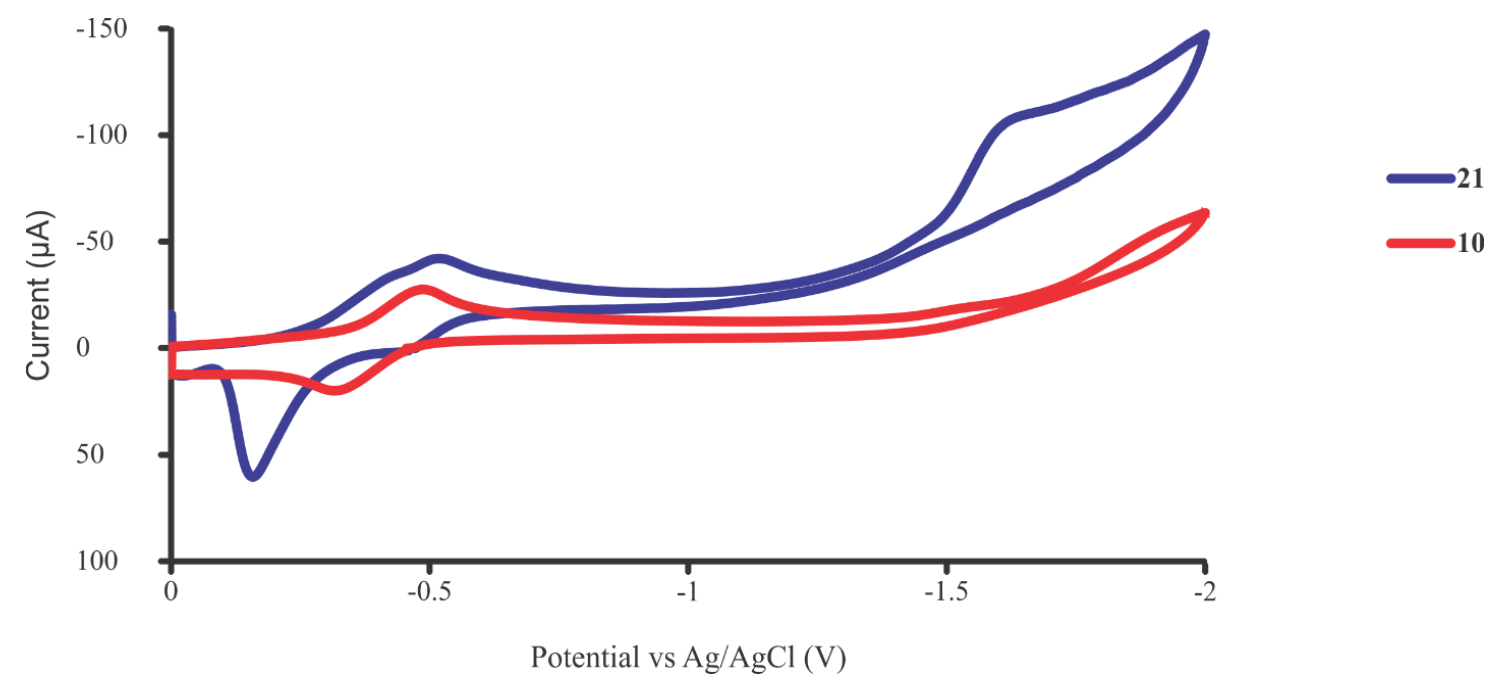

Figure 5-7. Comparison of 21 and 10 under catalytic HER conditions.

The dissolution of $\mathbf{1}$ in DMSO gave a red solution which then had its UV-vis recorded, Figure 5-8 top, black trace. Then triflic acid was added in sequential $30 \mu \mathrm{L}$ aliquots, with spectrums recorded after each addition, Figure 5-8 (for clarity, only select total additions are shown). During the course of the titration a color change was observed from red to yellow, as indicated in the blue shift of the $\lambda_{\max }$ from 480 to $460 \mathrm{~nm}$. To ensure that the endpoint was reached, the corrected absorbance of 480 and $460 \mathrm{~nm}$ vs acid addition was plotted until a plateau was achieved, Figure 5-8 bottom. 

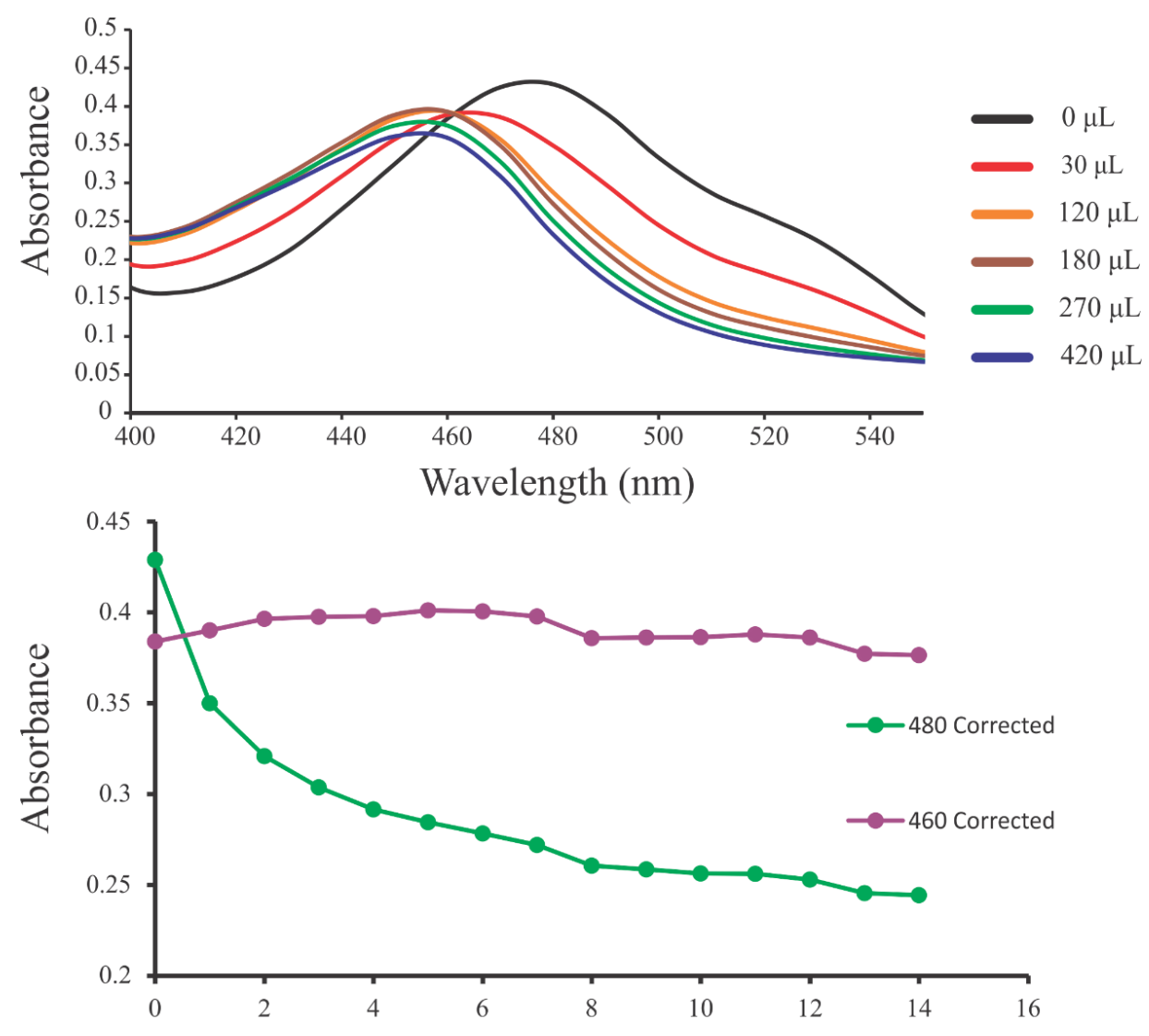

Addition

Figure 5-8. Selected UV-Vis spectra of 1 during titration (top) Corrected absorbance vs additions of acid for 480 and $460 \mathrm{~nm}$ (bottom).

This same experiment was perfomred for 21, Figure 5-9. As previously seen with 1, there is a blue shift in the $\lambda_{\max }$, from 490 to $450 \mathrm{~nm}$ and an isobestic point is observed at $460 \mathrm{~nm}$. These observations lead to the conclusion that restoration of the amine moiety allowed for protonation. However, it is important to note the volume of acid requisite to reach the saturation point of 21 of $660 \mu \mathrm{L}$ compared to that of $\mathbf{1}$ of $420 \mu \mathrm{L}$. This increase in acid addition is attributed again to the EWG of the alkoxy moiety leading to a decrease in overall basicity of the complex. 

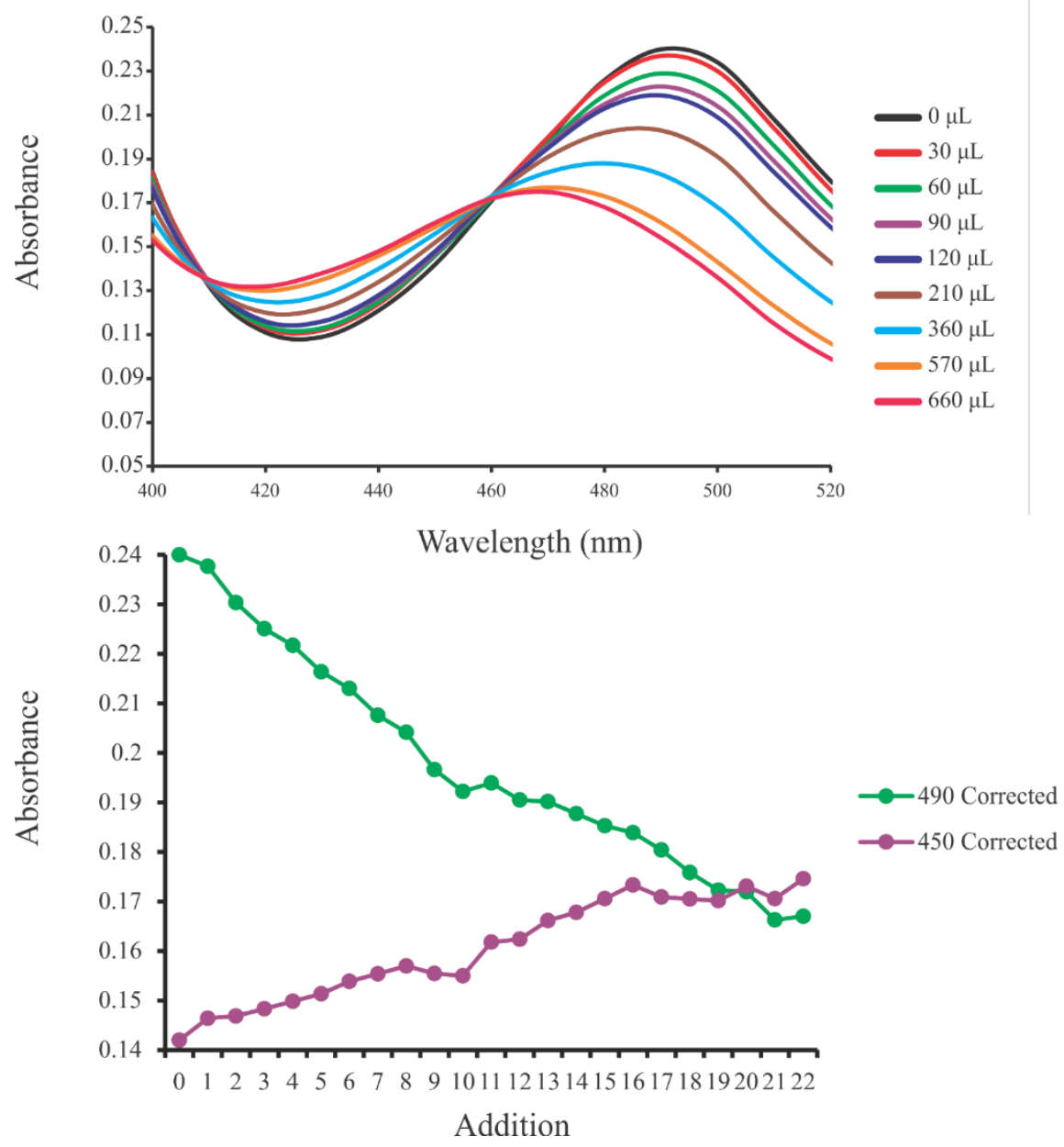

Figure 5-9. Selected UV-Vis spectra of 21 during titration (top) Corrected absorbance vs additions of acid for 490 and $450 \mathrm{~nm}$ (bottom).

Work done by Jain et al. ${ }^{76}$ and others ${ }^{35}$ highlighted the potential for NiBTSC complexes as homogeneous HER catalysts. Notably, the first step in the HER mechanism of NiBTSCs is reduction, not protonation. This change in mechanism could potentially allow for the employment of stronger EWG's without shutting down catalysis due to lack of basicity. These results lead to the use of NiATSM (2) as a parent compound, and synthesize 28 and 29 to investigate the effect of EWGs and electron donating groups (EDG) on heterogeneous HER catalysis, as described in Chapter 4. 


\subsubsection{Crystallography}

The solid state structures of $\mathbf{1 8}, \mathbf{1 9}, \mathbf{2 1}$, and $\mathbf{2 2}$ were determined by single crystal xray diffraction. The copper complexes 19 and 21 crystallized as a dark orange needle and an orange plate, respectively, in the space group $P-1$. The nickel complex 22 crystalized as an orange plate in the space group $P 2{ }_{1} / c$. The ligand, 18, crystallized as a yellow prism in the space group $P 2{ }_{1} / c$. All four complexes crystallize without inclusion of solvent molecules. Crystallographic data and structural refinement details are listed in the Chapter 2 Tables $2-3$ to $2-4$.

The ORTEP ${ }^{95}$ representations of 18, 19, 21, and 22, Figure 5-10 (A-D), show that each metal lays in a square planar $\mathrm{N}_{2} \mathrm{~S}_{2}$ donor environment. Selected bond distances and angles can be found in Table $5-1$. The $\mathrm{Ni}-\mathrm{N}$ and $\mathrm{Ni}-\mathrm{S}$ bonds are different from that of the $\mathrm{Cu}-\mathrm{N}$ and $\mathrm{Cu}-\mathrm{S}$ bonds, with the former having the shorter bond lengths. However, within a metal class, the chelation bonds are not statistically different. The $\mathrm{Cu}-\mathrm{N}$ bond distances of 1.959(2) and 1.967(2) for $\mathbf{1 9}$ are not different from those of CuATSM ${ }^{96}$ with corresponding lengths of $1.9584(16)$. This is also in good agreement with the $\mathrm{Cu}(\mathrm{II})$ compounds reported in Chapter 3. ${ }^{82}$ The $\mathrm{Cu}-\mathrm{S}$ bonds, of 2.2529(7) and 2.2795(7) for 21, and 2.2453(5) for that of CuATSM are also statistically equivalent. The same structural trend is observed for the $\mathrm{Ni}(\mathrm{II})$ complexes with the $\mathrm{Ni}-\mathrm{N}$ and $\mathrm{Ni}-\mathrm{S}$ bond lengths for all compounds. Within the ligand framework, all bond distances across the series regardless of the chelated metal are statistically equivalent. They are consistent with a conjugated $\pi-$ system of alternating single and double bonds. The bond distances in $\mathbf{1 9}$ of S1-C5, N1N2, and C1-C2, are 1.761(3), 1.362(3), and 1.477(3) A, respectively, which are typical of single $\mathrm{C}-\mathrm{S}, \mathrm{N}-\mathrm{N}$, and $\mathrm{C}-\mathrm{C}$ bonds. The remaining ligand framework bonds $\mathrm{N} 1-\mathrm{C} 1$ and $\mathrm{N} 2-$ 
C5 are shorter with distances of 1.293(3) and 1.318(3) Å respectively, consistent with C=N bond character. The sum of the four bond angles about the $\mathrm{Cu}$ is $358.33(0.12)^{\circ}$.

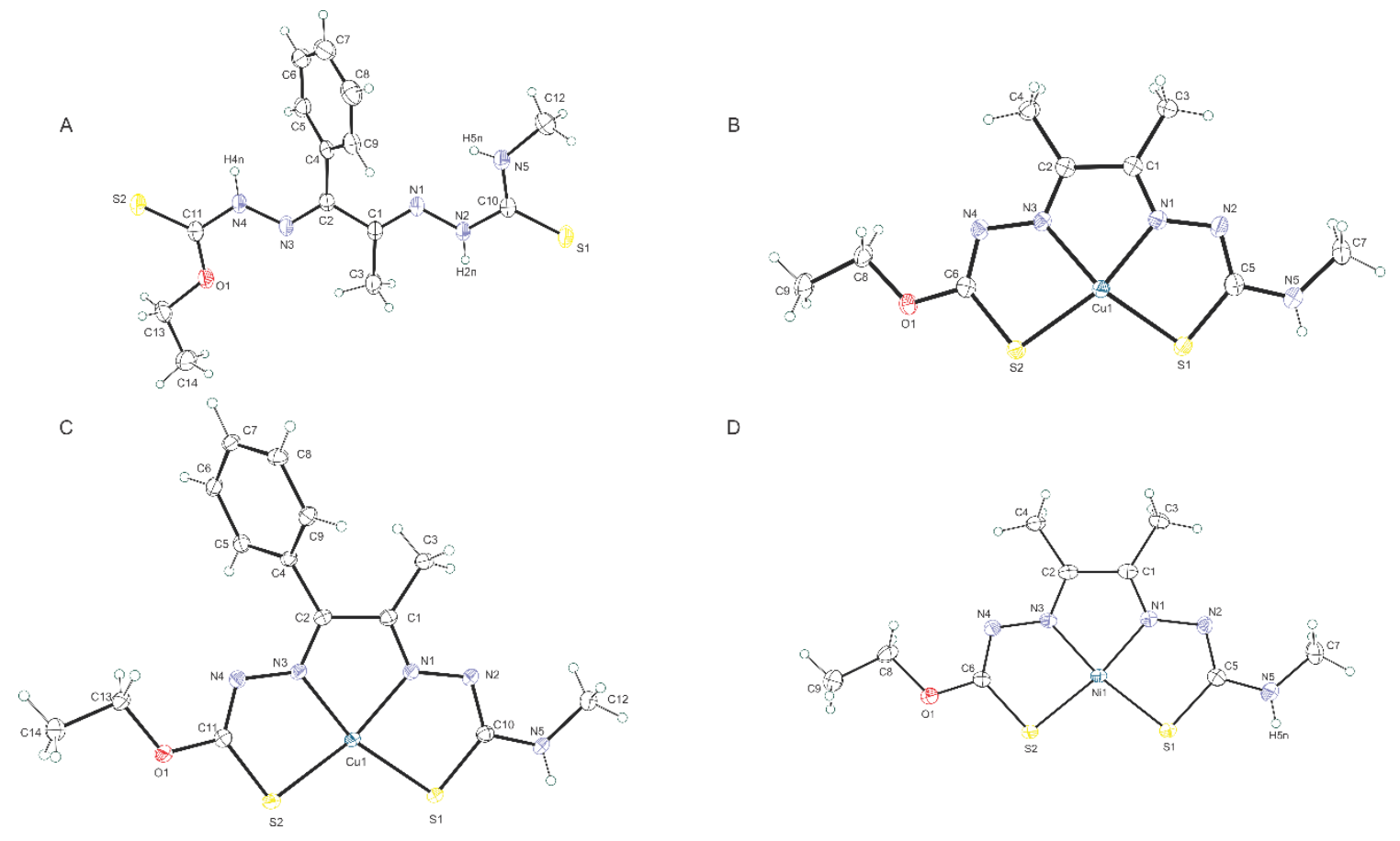

Figure 5-10. ORTEP $^{95}$ representations of 18(A), $19(\mathrm{~B}), 21(\mathrm{C})$, and $22(\mathrm{D})$.

All three chelates are rigorously planar. For complex 22, the best fit plane of all 18 non-hydrogen atoms has a standard deviation of $\pm 0.128 \AA$ with the largest deviation being $\pm 0.326 \AA$ for C9. For 19, the best fit plane for all 18 non-hydrogen atoms has a standard deviation of $\pm 0.067 \AA$ with the largest deviation of $\pm 0.116 \AA$ for C9. Excluding the phenyl ring in 21 (C5-C9) the best fit plane for all 18 non- hydrogen atoms has a standard deviation of $\pm 0.137 \AA$ with the largest deviation of $\pm 0.329 \AA$ for $\mathrm{C} 12$ (C7). The phenyl ring atoms of C5-C9 are planar within the ring, with a standard deviation of $\pm 0.013 \AA$ with the largest deviation of $\pm 0.01662 \AA$ for C5. The phenyl ring within the free ligand exhibits the same phenomenon with a standard deviation of $\pm 0.013 \AA$ and with the largest deviation of $\pm 0.01662 \AA$ for C5. 
Table 5-1. Selected bond distances and angles for 18, 19, 21, and 22.

\begin{tabular}{lllll}
\hline Bond Distance & $\mathbf{1 8}$ & $\mathbf{1 9}$ & $\mathbf{2 1}$ & $\mathbf{2 2}$ \\
M-N1 & N/A & $1.967(2)$ & $1.9755(14)$ & $1.868(3)$ \\
M-N3 & N/A & $1.959(2)$ & $1.9590(13)$ & $1.859(2)$ \\
M-S1 & N/A & $2.2529(7)$ & $2.2725(4)$ & $2.1554(8)$ \\
M-S2 & N/A & $2.2795(7)$ & $2.2871(5)$ & $2.1740(8)$ \\
S1-C5 $(C 10)^{a}$ & $1.6736(13)$ & $1.761(3)$ & $1.7584(17)$ & $1.760(3)$ \\
S2-C6 $(C 11)^{a}$ & $1.6568(14)$ & $1.758(3)$ & $1.7376(16)$ & $1.753(3)$ \\
O1-C6 $(C 11)^{a}$ & $1.3160(13)$ & $1.332(3)$ & $1.3339(19)$ & $1.334(4)$ \\
N1-C1 & $1.2898(17)$ & $1.293(3)$ & $1.298(2)$ & $1.303(4)$ \\
N1-N2 & $1.3660(15)$ & $1.362(3)$ & $1.3611(19)$ & $1.376(4)$ \\
N2-C5 $(C 10)^{a}$ & $1.3672(17)$ & $1.318(3)$ & $1.319(2)$ & $1.319(4)$ \\
N5-C5(C10) & $1.3251(18)$ & $1.338(3)$ & $1.331(2)$ & $1.338(4)$ \\
C1-C2 & $1.4778(18)$ & $1.477(3)$ & $1.472(2)$ & $1.473(4)$ \\
C1-C3 & $1.496(2)$ & $1.492(4)$ & $1.492(2)$ & $1.488(4)$ \\
\hline Bond Angles & & & & \\
\hline N3-M-N1 & N/A & $80.31(9)$ & $79.68(6)$ & $83.38(11)$ \\
N1-M-S1 & N/A & $84.58(6)$ & $83.31(4)$ & $87.18(8)$ \\
N3-M-S2 & N/A & $84.59(6)$ & $84.49(4)$ & $87.27(8)$ \\
S1-M-S2 & N/A & $108.85(3)$ & $111.359(16)$ & $102.15(3)$ \\
\hline
\end{tabular}

a) Numbers are different due to presence of benzene rings in $\mathbf{1 8}$ and $\mathbf{2 1}$.

\subsubsection{Electrochemistry}

The importance of electrochemical properties of the BTSC complexes has been well documented. The cyclic voltammograms of the free ligands and metal chelates can be found in Figure 5-11 (A-D). Notably, both the $\mathrm{Cu}$ and $\mathrm{Ni}$ derivatives display quasireversible events in their respective voltammograms. This is in contrast to the $\mathrm{Zn}$ and free ligand voltammograms, which display two irreversible reductions. As the chelated metal is varied, a periodic trend is observed with $\mathrm{Cu}$ have the most anodic events, followed by $\mathrm{Ni}$, then $\mathrm{Zn}$, and lastly the free ligand. The $\mathrm{Cu}^{\mathrm{II} / \mathrm{I}}$ couple is $0.57-0.63 \mathrm{~V}$ more anodic than that of the first redox event of the Ni class (ligand centered) and $1.28-1.36 \mathrm{~V}$ more anodic than the $\mathrm{Ni}{ }^{\mathrm{I} / \mathrm{I}}$ couple, Table $5-2$. The Ni ligand centered reduction is $0.41-0.42 \mathrm{~V}$ more anodic than that of the first $\mathrm{Zn}$ reduction and $0.55-0.68 \mathrm{~V}$ than the first free ligand 
reduction. Lastly the $\mathrm{Zn}$ stabilizes the ligand centered reductions by $0.14-0.26 \mathrm{~V}$ and 0.47 $-0.56 \mathrm{~V}$ more anodic than the respective free ligand events.
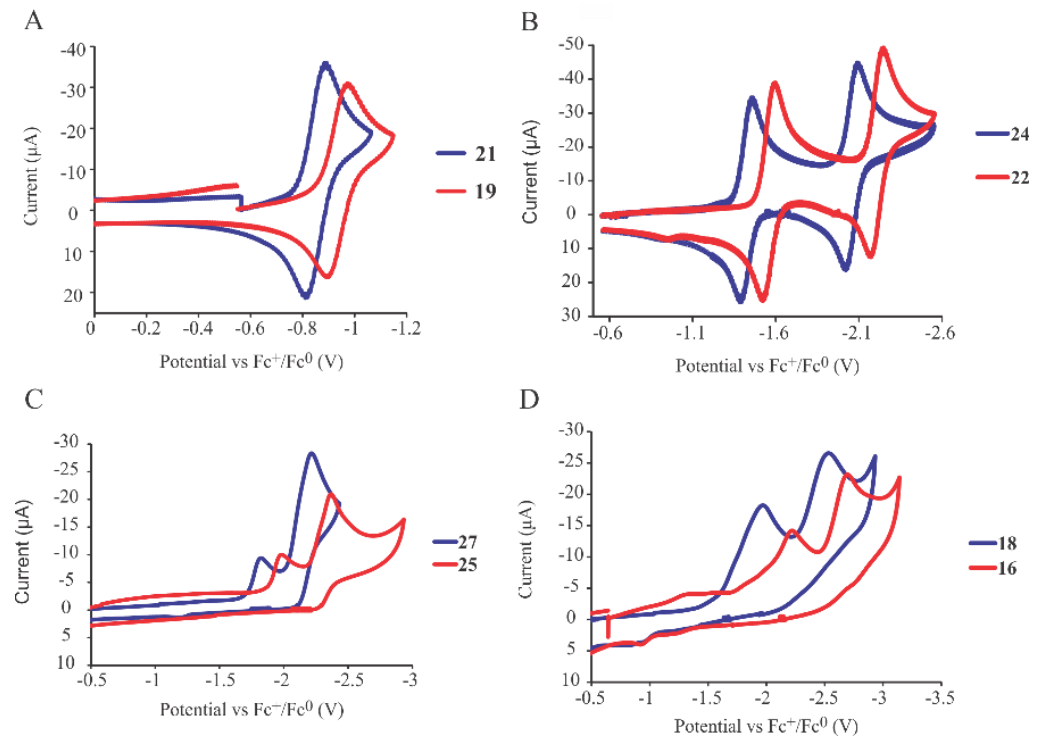

Figure 5-11. Cyclic voltammograms over lays of ligand framework $\mathbf{1 6}$ (red trace) and $\mathbf{1 8}$ (blue trace) with their respective chelates copper (A), nickel (B), zinc (C) and, free ligand (D).

Across the ligand series, there is a clear effect of electron donor ability from the $\mathrm{R}$ groups on reduction potential. The alkyl extension of R2 from ethyl to propyl resulted in a minimal or no effect on reduction potential. ${ }^{82}$ This is exemplified in the $0.01 \mathrm{~V}$ shifts for $\mathrm{Cu}$ complexes 19 and $\mathbf{2 0}$, and first free ligand reduction, $\mathbf{1 6}$ and $\mathbf{1 7}$, event and the $0.03 \mathrm{~V}$ shift for the second $\mathrm{Zn}$ reduction in $\mathbf{2 5}$ and $\mathbf{2 6}$. All other redox couples were unchanged by alkyl extension. However, the incorporation of a phenyl group in R1 caused a significant anodic shift in reduction potential for all compounds. For the $\mathrm{Cu}$ complexes 19 and 21, a $0.08 \mathrm{~V}$ anodic shift is observed. The most dramatic effect on the reduction potential is for the free ligand $\mathbf{1 6}$ and $\mathbf{1 8}$, which exhibited a $0.17 \mathrm{~V}$ anodic shift in potential.

In comparison with the previously reported ATSM and GTSM the copper derivatives are found to lay more anodic than both, with potentials of -1.11 and $-0.950 \mathrm{~V}$ 
respectively. This indicates that these compounds could have potential use in anti-cancer applications given the accessibility of their reduction potentials. This clearly demonstrates the ability to tune the reduction potential via the incorporation of an alkoxy moiety on only one side of the ligand framework cause the potential to lay in between that of the bis-amine and bis-alkoxy derivatives.

Table 5-2. $\mathrm{E}_{1 / 2}$ values for 16-27 $(\mathrm{V}) \mathrm{vs}^{\mathrm{Fc}} / \mathrm{Fc}$

\begin{tabular}{cccccccc}
\hline Ligand & $\mathrm{Cu}(\mathrm{II} / \mathrm{I})$ & $\mathrm{NiL} / \mathrm{NiL}^{-}$ & $\mathrm{Ni}(\mathrm{II} / \mathrm{I})$ & $\mathrm{ZnL}^{-} \mathrm{ZnL}^{-}$ & $\mathrm{ZnL}^{-} / \mathrm{ZnL}^{2-}$ & $\mathrm{L} / \mathrm{L}^{-}$ & $\mathrm{L}^{-} / \mathrm{L}^{2-}$ \\
\hline $\mathbf{1 6}$ & -0.930 & -1.56 & -2.21 & -1.98 & -2.37 & -2.23 & -2.70 \\
$\mathbf{1 7}$ & -0.940 & -1.56 & -2.22 & -1.98 & -2.40 & -2.24 & -2.70 \\
$\mathbf{1 8}$ & -0.850 & -1.42 & -2.12 & -1.83 & -2.22 & -1.97 & -2.53 \\
\hline
\end{tabular}

\subsubsection{Cell line Evaluation}

To investigate any potential therapeutic effects the compounds may have for anticancer, they were screened against the cancer cell line A549 and its healthy counterpart IMR-90. This work was conducted in collaboration with Dr. Paula Bates at the University of Louisville Medical School. All MTT assay experiments were performed by Dr. Sarah Andres. The MTT assay of ligand system 16, 17, and 18 are shown in Figure 5-12 and 513 (A and B), respectively. Since all compounds display redox activity, redox activity alone does not lead to enhanced therapeutic potential. The $\mathrm{Cu}$ complexes 19, 20, and 21 have respective GI50 values of $0.082,0.29$, and $0.065 \mu \mathrm{M}$ for A549, compared to >2.0, >2.0, and $1.3 \mu \mathrm{M}$ of the IMR-90 cells line, Table 5-3. The nickel derivatives, 22, 23, and 24, display larger GI50 values for both A549 and IMR90 cell lines, with no clear trend in selectivity. The difference in performance for the $\mathrm{Cu}$ and $\mathrm{Ni}$ complexes is attributed to the relatively accessible reduction potential of the $\mathrm{Cu}$ complexes as compared to $\mathrm{Ni}$, Table 52. With the copper chelates displaying substantially more anodic potentials, the $\mathrm{Cu}(\mathrm{II})$ core of 19 - 20 are capable of being reduced to $\mathrm{Cu}(\mathrm{I})$ intracellularly and thus have high 
bioactivity. This selectivity trend based on reduction potential not only explains the difference between our copper and nickel compounds but also previously reported ATSM derivatives, appendix Figure A62. It is clear that when both cell types are treated with CuATSM, a larger dose is required to see the anti-proliferation effects with the A549 and IMR90 cells. This result shows that even with the same chelated metal, $\mathrm{Cu}$, the $\mathrm{Cu}^{\mathrm{IIII}}$ reduction potential is crucial in creating a selective therapeutic.

Table 5-3. GI50 values for 16-27.

\begin{tabular}{lcccc}
\hline Ligand/Cell line GI50 $(\mu \mathrm{M})$ & $\mathrm{Cu}$ & $\mathrm{Ni}$ & $\mathrm{Zn}$ & Ligand \\
16 A549 & 0.082 & 3.0 & 0.59 & 0.60 \\
16 IMR90 & $>2.0$ & 5.3 & 0.68 & 1.1 \\
17 A549 & 0.29 & 10.1 & 0.45 & 0.47 \\
17 IM90 & $>2$ & 13 & 0.52 & $>2$ \\
18 A549 & 0.065 & 37 & 0.055 & 0.091 \\
18 IMR90 & 1.3 & $>100$ & 1.1 & $>2$ \\
\hline
\end{tabular}

To examine any potential trans-metallation and structural ligand effects both the free ligand and $\mathrm{Zn}$ complexes were evaluated for their potential therapeutic capabilities. It is evident from the MTT assays that the ligand framework can play a key role in biological activity. The free ligands $\mathbf{1 6}$ and $\mathbf{1 7}$ and $\mathrm{Zn}$ complexes $\mathbf{2 5}$ and $\mathbf{2 6}$ show lower therapeutic potential relative to their $\mathrm{Cu}$ analogues. The compounds $\mathbf{1 6}, \mathbf{1 7}, \mathbf{2 5}$, and 26 display improved therapeutic capabilities when they are compared to their corresponding Ni analogues. This is not the case however, for the free ligand 18 and $\mathrm{Zn}$ complex 27. The MTT assay profiles are nearly identical to their copper analog $\mathbf{2 1}$. This degree of similarity could suggest potential trans-metallation is occurring in the growth media and the copper complex is being generated in situ and is the active species. Should this have been the case then both $\mathbf{2 5}$ and $\mathbf{2 6}$ would have displayed similar profiles to their copper analogs, which they do not. Interestingly, all the free ligands $\mathbf{1 6}-\mathbf{1 8}$ display similar 
profiles to their $\mathrm{Zn}$ complexes $\mathbf{2 5} \mathbf{- 2 7}$. Thus any difference in the activity of the ligands and $\mathrm{Zn}$ compounds can be attributed to a structural effect. The major structural difference of $\mathbf{1 8}$ compared to $\mathbf{1 6}$ and $\mathbf{1 7}$ is the presence of the phenyl group in the backbone. It should be noted that 18, was not purified from the crude product, which may be contaminated with starting material, so some activity could be from the unreacted starting material. It is also possible that there was a difference in copper concentration in the culture media from the different lots of media employed during screening, leading to more extensive transmetallation in the evaluation of $\mathbf{1 8}$ and $\mathbf{2 7}$. To ensure all compounds are evaluated under identical copper concentrations, the media needs to be scrubbed with tetrathiomolybdate.

Interestingly, the phenyl structural component does not lead to activity in $\mathbf{2 4}$. The lack of activity of $\mathbf{2 4}$ can be attributed to its d-electron count. Compounds $\mathbf{2 1}$, and $\mathbf{2 7}$ after reduction, are $\mathrm{d}^{10}$ thus are kinetically labile however, even after reduction, $\mathbf{2 4}$ is only $\mathrm{d}^{9}$. In the kinetically labile $\mathbf{2 1}$ and $\mathbf{2 7}$, the metal is proposed to dissociate leaving the active ligand which has more conformation flexibility than the metal complexes. In $\mathbf{2 4}$ however, the ligand framework is forced to remain in the square planar chelate system and thus is not capable of achieving the required orientation for inhibition. An interesting possibility for a mechanism of action is that the ligand is the biologically active material and the metal plays the role of chaperone. If reduction potential was the dominant factor for potency, then it would be expected that $\mathbf{2 1}$ would display substantially higher activity compared to its more cathodic analogs $\mathbf{1 9}$ and $\mathbf{2 0}$. Given the similarity of their GI50 values, this is most likely not the case. However, if bioactivity was predominantly based on structure, then the nickel derivatives $\mathbf{2 2}-\mathbf{2 4}$ would be as active. The ligand itself also does not display equal 
or better activity to the copper derivatives. This led to the hypothesis that the square planar nature of the metal chelate enhances cellular uptake and the reduction potential then allows for selectivity. After reduction in the desired cell, the metal can be released leaving the free ligand to inhibit cellular respiration (Scheme 5-2).
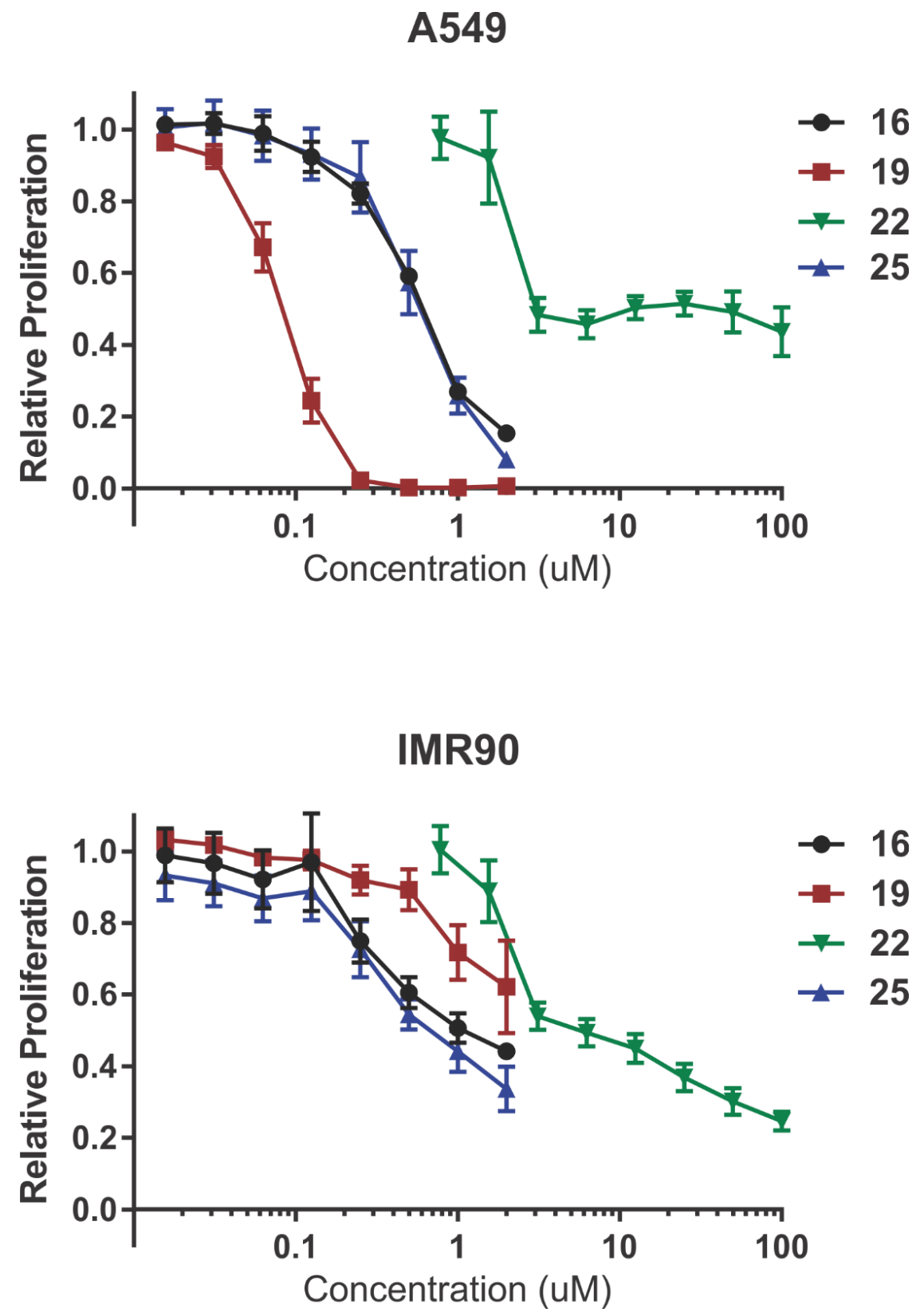

Figure 5-12. MTT assay results of cell lines A549 (top) and IMR90 (bottom) for the free ligand 16 (black trace), $\mathrm{Cu}$ complex 19 (red trace), Ni complex 22 (green trace) and $\mathrm{Zn}$ complex 25 (blue trace). 
A

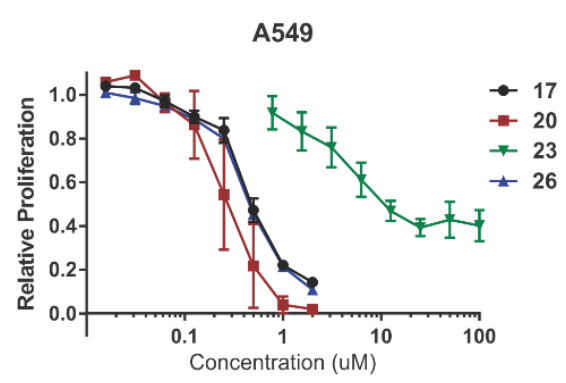

IMR90

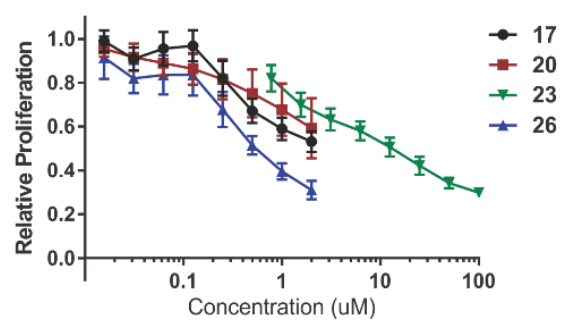

B
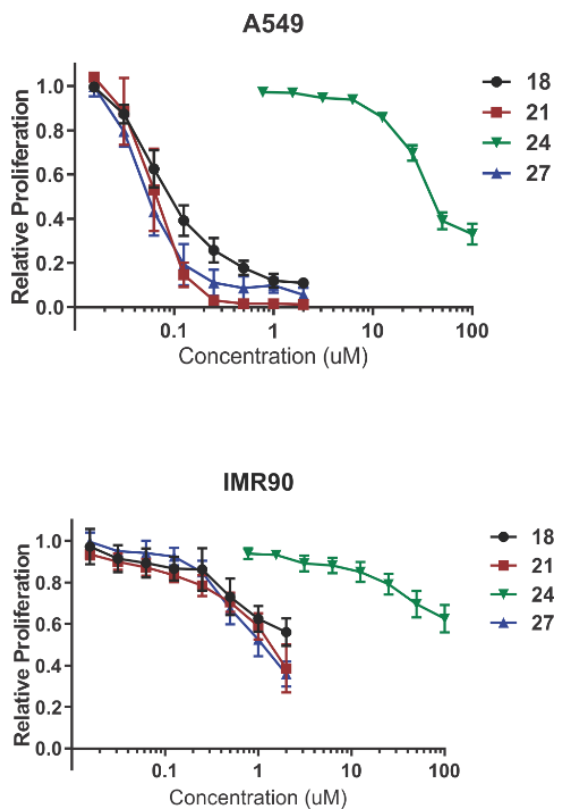

Figure 5-13. MTT assay results of cell lines A549 (top) and IMR90 (bottom) for the free ligands 17 (A) and 18 (B) (black trace), Cu complexes 20 (A) and 21 (B) (red trace), Ni complexes 23 (A) and 24 (B) (green trace) and $\mathrm{Zn}$ complexes 26 (A) and 27 (B) (blue trace).

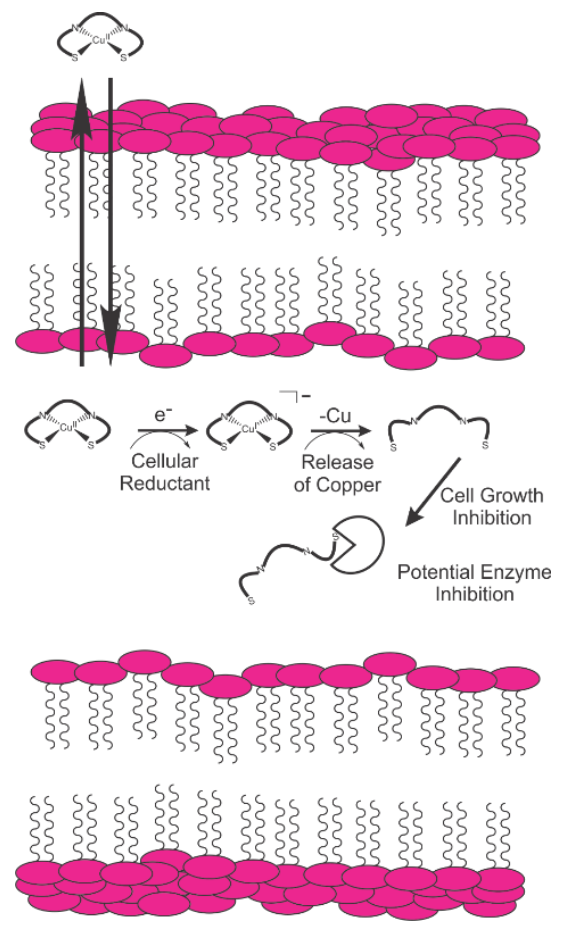

Scheme 5-2. Proposed mechanism of action for anti-cancer effects from copper chelates. 


\subsubsection{National Cancer Institute 60 Cell Line}

Compounds 19 and 21 were sent to the NIH to be examined with the National Cancer Institute 60 Cell Line (NCI-60) screening. The NCI-60 is a panel of a variety of carcinogenic cell lines used to examine compounds for their anti-cancer properties. Notably, the diversity of the carcinogenic cell lines employed allows for the comparison of tested compounds against known anti-cancer agents to identify a possible mechanism of action. The results, shown in Figure 5-14 and 5-15 respectively, show that these compounds are active against most of the tested cell lines with similar profiles, and in most cases were cytotoxic. It should be noted however that both compounds were completely inactive against all leukemia lines. The stark contrast in activity may be attributed to the difference in the types of cancer. The compounds were most active against cell lines derived from solid tumors, whereas leukemia is not. The cell lines derived from solid tumors may provide a more hypoxic environment that would allow the redox active compounds to operate as anti-cancer agents, whereas a lack of a hypoxic environment, such as in the leukemia cells, would prevent any cytotoxicity via redox active compounds. It should be noted that the cell lines derived from solid tumors are not grown under a hypoxic environment. Also, several tumor cell lines displayed resistance to our compounds. This resistance has yet to be fully understood, but elucidation of their differences relative to the rest of the solid tumors will provide valuable insight into the mode of action of these compounds.

\subsection{Conclusions}

A new ligand system and the corresponding nickel, copper and, zinc chelates were successfully synthesized. Incorporation of the alkoxy moiety allows tuning of the 
reduction potential to achieve values relevant for biological activity. Further, the effect of structure on activity was demonstrated with the improved potency of the phenyl derivative. The NCI-60 screening demonstrates that this new class of chelates are active against a wide variety of cell lines however, a clear mechanism of action is yet to be elucidated.

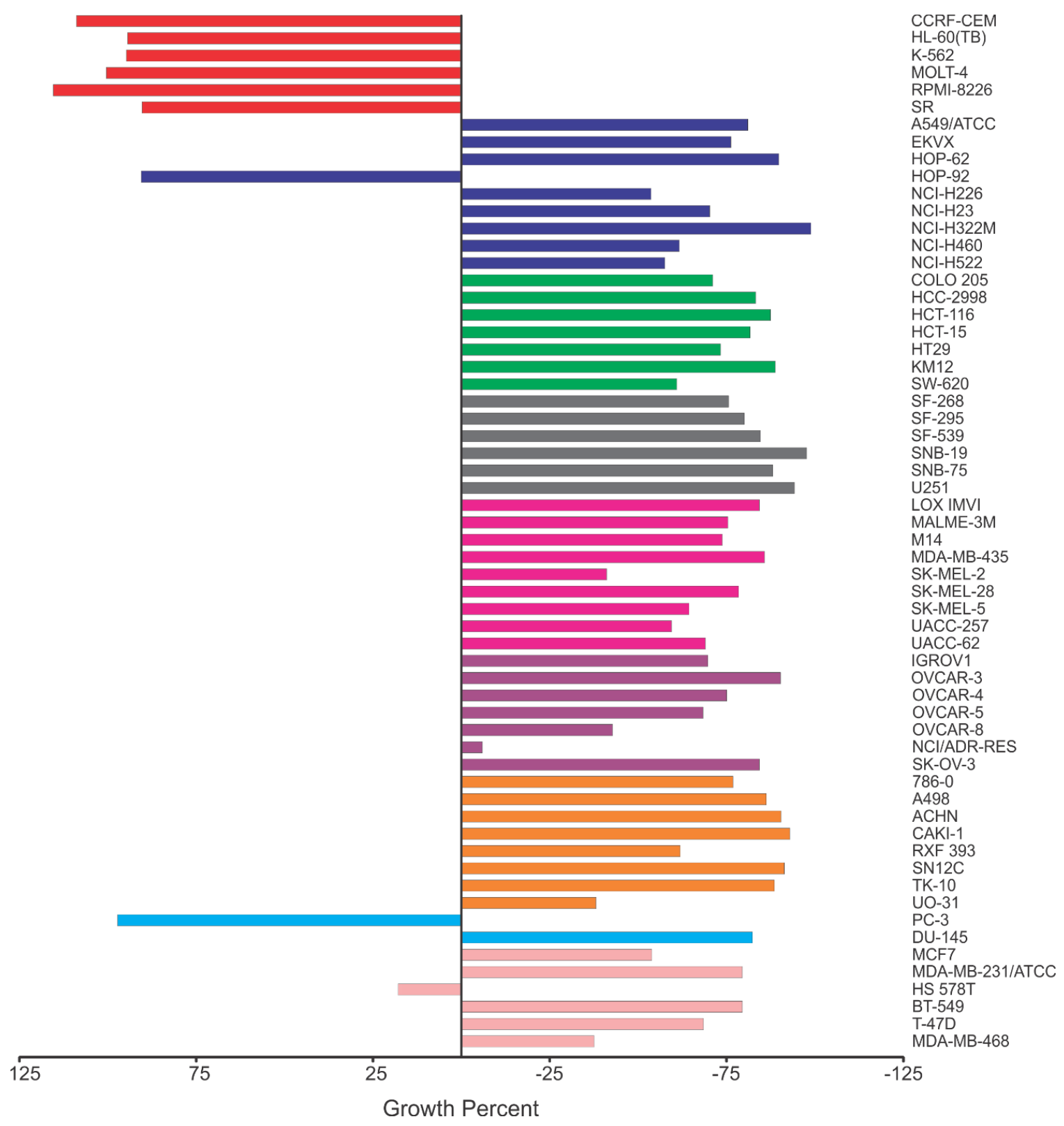

Figure 5-14. NCI-60 profile of 19 displaying the percent growth relative to the control. 


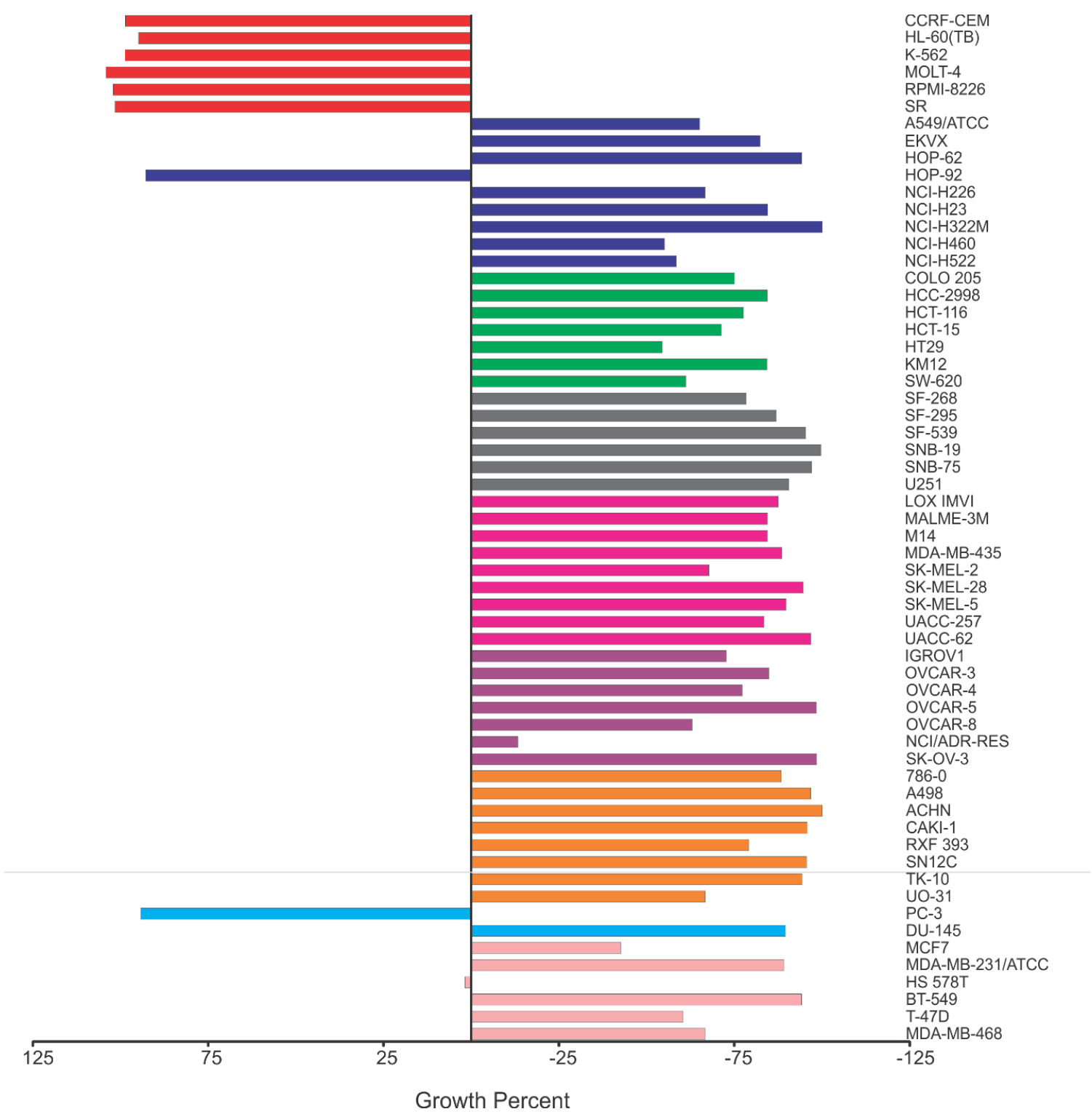

Figure 5-15. NCI-60 profile of 21 displaying the percent growth relative to the control. 


\section{CHAPTER VI}

\section{CONCLUSIONS}

The progress of BTSC's in therapeutics and diagnostics prompted the synthesis of alkoxy analogues to explore a new functional group and its effect on structure and reduction potentials. A total of six new ligands $(\mathbf{4}-\mathbf{9})$ and their copper chelates $(\mathbf{1 0}-\mathbf{1 5})$ were synthesized from bulk commodity chemicals with decent yields. Importantly the synthetic route uses alcohols, both primary and secondary alcohols for the starting material, which enables a variety of derivatives to be synthesized. The investigation into the structure revealed that all of the copper chelates $(\mathbf{1 0}-\mathbf{1 5})$ were analogous to CuATSM. Single crystal X-ray crystallography showed that these chelates are rigorously planar, a crucial facet to be capable of entering cells. Further the relative energy of the d-orbital manifold was found to be similar. However, the electrochemistry revealed a substantial anodic shift in the $\mathrm{Cu}^{\mathrm{II} / \mathrm{I}}$ potential of $\sim 250 \mathrm{mV}$ when comparing $\mathbf{1 0}$ to CuATSM. This large shift even puts these systems more anodic than CuGTSM, which makes them interesting as possible drug candidates. The observed shift allows for greater tuning of the electrochemistry of these types of ligand frameworks, which has been shown to be important to the function of the BTSC class. However, by removing the two hydrogen bond donors that are present in the ATSM framework, the solubility was significantly altered. These alkoxy systems could only be dissolved in halogenated organic solvents, like DCM, effectively neutralizing any potential therapeutic properties that require dissolution prior to treatment. If a suspension or oral gavage could be employed these systems can be evaluated for their drug like 
properties. It should be noted that the reduction potential of these systems is only modulated within a three carbon chain length, meaning effectively the solubility could be restored with a functionalized alcohol without effecting the redox couple. One possible synthetic route that could be explored to implement these more exotic alcohols is transesterification much like Que and co-workers demonstration of the applicability of transamination. Further the alkoxy class of ligands failed to act as HER catalysts due to the poor basicity of the ligand framework. This result demonstrated the limit of electron withdrawing that future investigations could employ to attempt to minimize the overpotential of such catalysts, a central step in rational design.

The data from the alkoxy systems $(\mathbf{4}-\mathbf{1 5})$ prompted the restoration of the pendant amine on one side of the ligand to create a hybrid system. Notably, this improved solubility, catalysis performance, and cathodically shifted the $\mathrm{Cu}^{\mathrm{II/I}}$ potential relative to the alkoxy functionality. Ultimately though, the hybrid system failed to perform adequately as an HER catalyst. However, this again showed that while this system was capable of producing $\mathrm{H}_{2}$, the electron withdrawing functional group prevents sufficient performance, leading to the focus of improvement on traditional BTSC complexes.

Three Ni BTSCs were immobilized on a glassy carbon electrode for heterogeneous HER catalysis. Catalysts $\mathbf{2}$ and $\mathbf{2 8}$ displayed similar HER performance, whereas 29, was substantially different. This difference in performance is attributed to the solid state intermolecular packing. Single crystal X-ray crystallography provided essential insight to how the complexes interacted in the solid state. The two rigorously planar systems, $\mathbf{2}$ and $\mathbf{2 8}$ behaved and packed in nearly identical fashions with closer intermolecular contacts less than $3.60 \AA$ A. The fluorinated groups of $\mathbf{2 9}$ prevented the molecules from forming close 
contacts with one another and the electrode surface. Thus inhibiting electron transport and leading to larger overpotentials and charge transfer resistance. Whereas the planar derivatives $\mathbf{2}$ and $\mathbf{2 8}$ can undergo dynamic re-arrangement during reductive cycling to form a better contact to the electrode surface that improves overall performance. Raman spectroscopy further revealed that $\mathbf{2 9}$ was lost from the electrode surface during reductive cycling.

The significant improvement after conditioning is vital to further enhancing overall catalyst performance of these systems. Both $\mathbf{2}$ and $\mathbf{2 8}$ display remarkable stability on the electrode surface and retained activity after extended cycling. The remarkable longevity without a covalent linker is crucial in development of these systems as the dynamic rearrangement is necessary for catalyst improvement and would be impossible if covalently linked to the electrode surface. A potential improvement could be made by incorporation of a molecule that facilitates electron transfer to further lower the charge transfer resistance for $\mathbf{2}$ and $\mathbf{2 8}$. For 29, the poor packing and lack of stability prevented an adequate analysis of electron withdrawing groups on heterogeneous catalysis for NiBTSCs. Thus synthesis of a novel derivative that can form close contacts and longevity on the electrode surface as displayed by $\mathbf{2}$ and $\mathbf{2 8}$, is paramount to further catalyst development.

The hybrid system failed for HER catalysis but the anodically shifted $\mathrm{Cu}^{\mathrm{I} / \mathrm{I}}$ reduction potential and improved solubility due to restoration of the hydrogen bond donor amine, indicated potential anti-cancer properties. A series of ligands $(\mathbf{1 6}-\mathbf{1 8})$ were synthesized and chelated with metals $\mathrm{Cu}(\mathbf{1 9}-\mathbf{2 1}), \mathrm{Ni}(\mathbf{2 2}-\mathbf{2 4})$, and $\mathrm{Zn}(\mathbf{2 5}-\mathbf{2 7})$ to give a total of 12 new compounds, which were each screened for therapeutic potential via an MTT assay. The results of the MTT assay point to the copper derivative being the most 
potent and selective between carcinogenic cells and healthy. Interestingly these results show that while reduction potential is crucial for activity and selectivity, there is an emerging importance of structure. The more active systems of the copper, zinc, and free ligand are deemed capable of becoming the free ligand (or are already) when in the cell. The copper system is capable of being reduced to $\mathrm{Cu}(\mathrm{I})$ which is kinetically labile, like zinc. Notably, the reduction potential of the zinc system is not capable of being achieved intracellularly, meaning the activity observed from zinc is purely structural. The same argument however does translate to the nickel derivative. The exception is that even if reduction was achieved for the nickel system, it would still be kinetically inert, so only structural effects of the chelate are observed. This explains why the zinc and free ligand have such similar profiles and the nickel performs poorly. Further, this explains the enhanced selectivity and potency of the copper complexes. The chelated form allows for facile diffusion across membranes, and the copper is only released when reduced. Additionally, if the reduction potential was the dominant factor in effectiveness, then $\mathbf{2 1}$ should show a significant increase in performance relative to 19. Although 21 does display a minor decrease in concentration required to achieve a GI 50, it is minimal considering the larger anodic shift. Additionally, $\mathbf{1 9}$ and $\mathbf{2 1}$ were screened against the 60 cell lines in the NCI 60 assay. Both systems exhibited a similar profile with high activity and cytotoxicity against a wide variety of cell lines derived from solid tumors. The leukemia cell lines remained unaffected by these complexes, providing valuable insight that can help elucidate a clear mechanism. These results are the first to demonstrate that the structure of the ligand and the $\mathrm{Cu}^{\mathrm{II} / \mathrm{I}}$ redox couple together lead to selectivity and activity. This will 
allow for better design of new systems to exploit the metal as the chaperone, but tuning the ligand to act as the drug.

Further, it has been demonstrated in the literature that BTSCs are substrates for MDR-1 due to their hydrogen bond donor pendant amine. It is plausible that tuning the reduction potential with the alkoxy functionality led to enhanced potency of these systems via inhibiting specificity for MDR-1. A binding affinity assay comparing ATSM to the hybrid could prove this, and thus further render the previous alkoxy complexes, $10-15$ desirable for therapeutics with lack of hydrogen bond donors and more anodic $\mathrm{Cu}^{\mathrm{I} / \mathrm{I}}$ potentials. However, solubility needs to be increased without restoration of hydrogen bond donors so as not to make the modified system a substrate for MDR-1. 


\section{REFERENCES}

1. Gardner, T. S.; Smith, F. A.; Wenis, E.; Lee, J., The Chemotherapy of Tuberculosis. III. Thiosemicarbazide Derivatives. J. Am. Chem. Soc. 1952, 74, 2106-2107.

2. Gardner, T. S.; Smith, F. A.; Wenis, E.; Lee, J., The Synthesis of Compounds for the Chemotherapy of Tuberculosis. I. Heterocyclic Thiosemicarbazide Derivatives. J. Org. Chem 1951, 16, 1121-1125.

3. $\quad$ Adsule, S.; Barve, V.; Chen, D.; Ahmed, F.; Dou, Q. P.; Padhye, S.; Sarkar, F. H., Novel Schiff Base Copper Complexes of Quinoline-2 Carboxaldehyde as Proteasome Inhibitors in Human Prostate Cancer Cells. J. Med. Chem. 2006, 49, 7242-7246.

4. $\quad$ Kowol, C. R.; Berger, R.; Eichinger, R.; Roller, A.; Jakupec, M. A.; Schmidt, P. P.; Arion, V. B.; Keppler, B. K., Gallium(III) and Iron(III) Complexes of $\alpha-\mathrm{N}-H e t e r o c y c l i c$ Thiosemicarbazones: Synthesis, Characterization, Cytotoxicity, and Interaction with Ribonucleotide Reductase. J. Med. Chem. 2007, 50, 1254-1265.

5. Raichurkar, A. V.; Kulkarni, V. M., Understanding the Antitumor Activity of Novel Hydroxysemicarbazide Derivatives as Ribonucleotide Reductase Inhibitors Using CoMFA and CoMSIA. J. Med. Chem. 2003, 46, 4419-4427.

6. Zhang, J., Preparation, Characterization and Application of Thiosemicarbazide Grafted Multiwalled Carbon Nanotubes for Solid-Phase Extraction of $\mathrm{Cd}(\mathrm{II}), \mathrm{Cu}(\mathrm{II})$ and $\mathrm{Pb}$ (II) in Environmental Samples. J. Environ. Sci. 2013, 25, 2331-2337.

7. $\quad$ Hickey, J. L.; Lim, S.; Hayne, D. J.; Paterson, B. M.; White, J. M.; Villemagne, V. L.; Roselt, P.; Binns, D.; Cullinane, C.; Jeffery, C. M.; Price, R. I.; Barnham, K. J.; Donnelly, P. S., Diagnostic Imaging Agents for Alzheimer's Disease: Copper Radiopharmaceuticals that Target A $\beta$ Plaques. J. Am. Chem. Soc. 2013, 135, 16120-16132.

8. $\quad$ West, D. X.; Ives, J. S.; Bain, G. A.; Liberta, A. E.; Valdés-Martínez, J.; Ebert, K. H.; Hernández-Ortega, S., Copper(II) and Nickel(II) Complexes of 2,3-butanedione Bis(N(3)Substituted Thiosemicarbazones). Polyhedron 1997, 16, 1895-1905.

9. Beraldo, H.; Boyd, L. P.; West, D. X., Copper(II) and Nickel(II) Complexes of Glyoxaldehyde Bis $\{\mathrm{N}(3)-S u b s t i t u t e d$ Thiosemicarbazones $\}$. Transition Met. Chem. 1997, 23, 67-

71.

10. Haddad, A. Z.; Garabato, B. D.; Kozlowski, P. M.; Buchanan, R. M.; Grapperhaus, C. A., Beyond Metal-Hydrides: Non-Transition-Metal and Metal-Free Ligand-Centered Electrocatalytic Hydrogen Evolution and Hydrogen Oxidation. J. Am. Chem. Soc. 2016, 138, 7844-7847.

11. King, A. P.; Gellineau, H. A.; Ahn, J.-E.; MacMillan, S. N.; Wilson, J. J., Bis(thiosemicarbazone) Complexes of Cobalt(III). Synthesis, Characterization, and Anticancer Potential. Inorg. Chem. 2017, 56, 6609-6623.

12. Tatiana, S.; Renaud, H.; Julien, M.; Mireille, A.; Bruno, F.; Michel, G.; Marius, R.; Maylis, O., Influence of the Metal Ion on the Electrocatalytic Hydrogen Production by a Thiosemicarbazone Palladium Complex. Eur. J. Inorg. Chem. 2018, 2018, 2259-2266.

13. Dearling, J. L.; Lewis, J. S.; Mullen, G. E.; Welch, M. J.; Blower, P. J., Copper Bis(thiosemicarbazone) Complexes as Hypoxia Imaging Agents: Structure-Activity Relationships. J. Biol. Inorg. Chem. 2002, 7, 249-259.

14. Betts, H. M.; Barnard, P. J.; Bayly, S. R.; Dilworth, J. R.; Gee, A. D.; Holland, J. P., Controlled Axial Coordination: Solid-Phase Synthesis and Purification of Metallo-

Radiopharmaceuticals. Angew. Chem. Int. Ed. 2008, 47, 8416-8419. 
15. Holland, J. P.; Aigbirhio, F. I.; Betts, H. M.; Bonnitcha, P. D.; Burke, P.; Christlieb, M.; Churchill, G. C.; Cowley, A. R.; Dilworth, J. R.; Donnelly, P. S.; Green, J. C.; Peach, J. M.; Vasudevan, S. R.; Warren, J. E., Functionalized Bis(thiosemicarbazonato) Complexes of Zinc and Copper: Synthetic Platforms Toward Site-Specific Radiopharmaceuticals. Inorg. Chem. 2007, 46, 465-485.

16. Holland, J. P.; Green, J. C.; Dilworth, J. R., Probing the Mechanism of Hypoxia Selectivity of Copper Bis(thiosemicarbazonato) Complexes: DFT Calculation of Redox Potentials and Absolute Acidities in Solution. Dalton Trans. 2006, 783-794.

17. Holland, J. P.; Barnard, P. J.; Collison, D.; Dilworth, J. R.; Edge, R.; Green, J. C.; McInnes, E. J., Spectroelectrochemical and Computational Studies on the Mechanism of Hypoxia Selectivity of Copper Radiopharmaceuticals. Chem. Eur. J. 2008, 14, 5890-5907.

18. Christlieb, M.; Struthers, H. S. R.; Bonnitcha, P. D.; Cowley, A. R.; Dilworth, J. R., The Exocyclic Functionalisation of Bis(thiosemicarbazonate) Complexes of Zinc and Copper: The Synthesis of Monomeric and Dimeric Species. Dalton Trans. 2007, 5043-5054.

19. Paterson, B. M.; Karas, J. A.; Scanlon, D. B.; White, J. M.; Donnelly, P. S., Versatile New Bis(thiosemicarbazone) Bifunctional Chelators: Synthesis, Conjugation to Bombesin(7-14)$\mathrm{NH}_{2}$, and Copper-64 Radiolabeling. Inorg. Chem. 2010, 49, 1884-1893.

20. Cortezon-Tamarit, F.; Sarpaki, S.; Calatayud, D. G.; Mirabello, V.; Pascu, S. I., Applications of "Hot" and "Cold" Bis(thiosemicarbazonato) Metal Complexes in Multimodal Imaging. Chem. Rec. 2016, 16, 1380-1397.

21. Pascu, S. I.; Waghorn, P. A.; Conry, T. D.; Betts, H. M.; Dilworth, J. R.; Churchill, G. C.; Pokrovska, T.; Christlieb, M.; Aigbirhio, F. I.; Warren, J. E., Designing Zn(II) and Cu(II) Derivatives as Probes for In Vitro Fluorescence Imaging. Dalton Trans 2007, 4988-4997.

22. Kenche, V. B.; Barnham, K. J., Alzheimer's Disease \& Metals: Therapeutic Opportunities. Br. J. Pharmacol. 2011, 163, 211-219.

23. Hung, L. W.; Villemagne, V. L.; Cheng, L.; Sherratt, N. A.; Ayton, S.; White, A. R.; Crouch, P. J.; Lim, S.; Leong, S. L.; Wilkins, S.; George, J.; Roberts, B. R.; Pham, C. L.; Liu, X.; Chiu, F. C.; Shackleford, D. M.; Powell, A. K.; Masters, C. L.; Bush, A. I.; O'Keefe, G.; Culvenor, J. G.; Cappai, R.; Cherny, R. A.; Donnelly, P. S.; Hill, A. F.; Finkelstein, D. I.; Barnham, K. J., The hypoxia Imaging Agent Cu ${ }^{\mathrm{II}}(\mathrm{ATSM})$ is Neuroprotective and Improves Motor and Cognitive Functions in Multiple Animal Models of Parkinson's Disease. J. Exp. Med. 2012, 209, 837-54.

24. Roberts, B. R.; Lim, N. K.; McAllum, E. J.; Donnelly, P. S.; Hare, D. J.; Doble, P. A.; Turner, B. J.; Price, K. A.; Lim, S. C.; Paterson, B. M.; Hickey, J. L.; Rhoads, T. W.; Williams, J. R.; Kanninen, K. M.; Hung, L. W.; Liddell, J. R.; Grubman, A.; Monty, J.-F.; Llanos, R. M.; Kramer, D. R.; Mercer, J. F.; Bush, A. I.; Masters, C. L.; Duce, J. A.; Li, Q.-X.; Beckman, J. S.; Barnham, K. J.; White, A. R.; Crouch, P. J., Oral Treatment with Cu (atsm) Increases Mutant SOD1 In Vivo but Protects Motor Neurons and Improves the Phenotype of a Transgenic Mouse Model of Amyotrophic Lateral Sclerosis. J. Neurosci. 2014, 34, 8021-8031.

25. McAllum, E. J.; Roberts, B. R.; Hickey, J. L.; Dang, T. N.; Grubman, A.; Donnelly, P. S.; Liddell, J. R.; White, A. R.; Crouch, P. J., Zn ${ }^{\mathrm{II}}$ (ATSM) is Protective in Amyotrophic Lateral Sclerosis Model Mice via a Copper Delivery Mechanism. Neurolbiol. Dis. 2015, 81, 20-24.

26. Limited, C. M. D. P., Phase 1 Dose Escalation and PK Study of Cu(II)ATSM in ALS/MND. ClinicalTrials.gov [Internet]: Bethesda (MD): National Library of Medicine (US), 2016.

27. Bonnitcha, P. D.; Vāvere, A. L.; Lewis, J. S.; Dilworth, J. R., In Vitro and In Vivo Evaluation of Bifunctional Bisthiosemicarbazone ${ }^{64} \mathrm{Cu}$-Complexes for the Positron Emission Tomography Imaging of Hypoxia. J. Med. Chem. 2008, 51, 2985-2991. 
28. Ikawa, M.; Okazawa, H.; Kudo, T.; Kuriyama, M.; Fujibayashi, Y.; Yoneda, M., Evaluation of Striatal Oxidative Stress in Patients with Parkinson's Disease Using $\left[{ }^{62} \mathrm{Cu}\right]$ ATSM PET. Nucl. Med. Biol. 2011, 38, 945-951.

29. Ikawa, M.; Okazawa, H.; Arakawa, K.; Kudo, T.; Kimura, H.; Fujibayashi, Y.;

Kuriyama, M.; Yoneda, M., PET Imaging of Redox and Energy States in Stroke-Like Episodes of MELAS. Mitochondrion 2009, 9, 144-148.

30. Fujibayashi, Y.; Taniuchi, H.; Yonekura, Y.; Ohtani, H.; Konishi, J.; Yokoyama, A., Copper-62-ATSM: A New Hypoxia Imaging Agent with High Membrane Permeability and Low Redox Potential. J. Nucl. Med. 1997, 38, 1155-1160.

31. Vavere, A. L.; Lewis, J. S., Cu-ATSM: A Radiopharmaceutical for the PET Imaging of Hypoxia. Dalton Trans. 2007, 4893-4902.

32. Xie, D.; King, T. L.; Banerjee, A.; Kohli, V.; Que, E. L., Exploiting Copper Redox for ${ }^{19}$ F Magnetic Resonance-Based Detection of Cellular Hypoxia. J. Am. Chem. Soc. 2016, 138, 2937-2940.

33. Cowley, A. R.; Dilworth, J. R.; Donnelly, P. S.; Heslop, J. M.; Ratcliffe, S. J.,

Bifunctional Chelators for Copper Radiopharmaceuticals: The Synthesis of [Cu(ATSM)-Amino Acid] and [Cu(ATSM)-Octreotide] Conjugates. Dalton Trans. 2007, 209-217.

34. Haddad, A. Z.; Cronin, S. P.; Mashuta, M. S.; Buchanan, R. M.; Grapperhaus, C. A., Metal-Assisted Ligand-Centered Electrocatalytic Hydrogen Evolution upon Reduction of a Bis(thiosemicarbazonato)Cu(II) Complex. Inorg. Chem. 2017, 56, 11254-11265.

35. Straistari, T.; Fize, J.; Shova, S.; Réglier, M.; Artero, V.; Orio, M., A

Thiosemicarbazone-Nickel(II) Complex as Efficient Electrocatalyst for Hydrogen Evolution. ChemCatChem 2017, 9, 2262-2268.

36. Liu, J.; Hajibeigi, A.; Ren, G.; Lin, M.; Siyambalapitiyage, W.; Liu, Z.; Simpson, E.; Parkey, R. W.; Sun, X.; Oz, O. K., Retention of the radiotracers 64Cu-ATSM and 64Cu-PTSM in human and murine tumors is influenced by MDR1 protein expression. J Nucl Med 2009, 50, 1332-9.

37. Young, H.; Carnochan, P.; Zweit, J.; Babich, J.; Cherry, S.; Ott, R., Evaluation of copper(II)-pyruvaldehyde bis (N-4-methylthiosemicarbazone) for tissue blood flow measurement using a trapped tracer model. Eur J Nucl Med 1994, 21, 336-41.

38. Cater, M. A.; Pearson, H. B.; Wolyniec, K.; Klaver, P.; Bilandzic, M.; Paterson, B. M.; Bush, A. I.; Humbert, P. O.; La Fontaine, S.; Donnelly, P. S.; Haupt, Y., Increasing Intracellular Bioavailable Copper Selectively Targets Prostate Cancer Cells. ACS Chem. Biol. 2013, 8, 16211631.

39. Palanimuthu, D.; Shinde, S. V.; Somasundaram, K.; Samuelson, A. G., In Vitro and in Vivo Anticancer Activity of Copper Bis(thiosemicarbazone) Complexes. J. Med. Chem. 2013, 56, $722-734$.

40. $\quad$ Hickey, J. L.; James, J. L.; Henderson, C. A.; Price, K. A.; Mot, A. I.; Buncic, G.; Crouch, P. J.; White, J. M.; White, A. R.; Smith, T. A.; Donnelly, P. S., Intracellular Distribution of Fluorescent Copper and Zinc Bis(thiosemicarbazonato) Complexes Measured with Fluorescence Lifetime Spectroscopy. Inorg. Chem. 2015, 54, 9556-9567.

41. Crim, J. A.; Petering, H. G., The Antitumor Activity of Cu(II)KTS, the Copper(II) Chelate of 3-Ethoxy-2-oxobutyraldehyde Bis(thiosemicarbazone). Cancer Res. 1967, 27, 12781285 .

42. Donnelly, P. S.; Liddell, J. R.; Lim, S.; Paterson, B. M.; Cater, M. A.; Savva, M. S.; Mot, A. I.; James, J. L.; Trounce, I. A.; White, A. R.; Crouch, P. J., An Impaired Mitochondrial Electron Transport Chain Increases Retention of the Hypoxia Imaging Agent Diacetylbis(4methylthiosemicarbazonato)Copper ${ }^{\text {II }}$. Proc. Natl. Acad. Sci. U. S. A. 2012, 109, 47-52. 43. vandenBerghe, Peter V E.; Folmer, Dineke E.; Malingré, Helga E M.; vanBeurden, E.; Klomp, Adriana E M.; vandeSluis, B.; Merkx, M.; Berger, R.; Klomp, Leo W J., Human Copper 
Transporter 2 is Localized in Late Endosomes and Lysosomes and Facilitates Cellular Copper Uptake. Biochem. J. 2007, 407, 49-59.

44. Hurwitz, A. A.; Foster, B. A.; Allison, J. P.; Greenberg, N. M.; Kwon, E. D., The TRAMP Mouse as a Model for Prostate Cancer. Curr. Protoc. Immunol. 2001, 45, 20.5.120.5.23.

45. Suttie, A.; Nyska, A.; Haseman, J. K.; Moser, G. J.; Hackett, T. R.; Goldsworthy, T. L., A Grading Scheme for the Assessment of Proliferative Lesions of the Mouse Prostate in the TRAMP Model. Toxicol Pathol 2003, 31, 31-8.

46. Sunderman, F. W., Jr., The Influence of Zinc on Apoptosis. Ann Clin Lab Sci 1995, 25, 134-42.

47. Chen, D.; Cui, Q. C.; Yang, H.; Barrea, R. A.; Sarkar, F. H.; Sheng, S.; Yan, B.; Reddy, G. P.; Dou, Q. P., Clioquinol, a Therapeutic Agent for Alzheimer's Disease, has ProteasomeInhibitory, Androgen Receptor-Suppressing, Apoptosis-Inducing, and Antitumor Activities in Human Prostate Cancer Cells and Xenografts. Cancer Res 2007, 67, 1636-44.

48. Chen, D.; Cui, Q. C.; Yang, H.; Dou, Q. P., Disulfiram, a Clinically used Anti-

Alcoholism Drug and Copper-Binding Agent, Induces Apoptotic Cell Death in Breast Cancer Cultures and Xenografts via Inhibition of the Proteasome Activity. Cancer Res 2006, 66, 1042533.

49. Cater, M. A.; Haupt, Y., Clioquinol Induces Cytoplasmic Clearance of the X-Linked Inhibitor of Apoptosis Protein (XIAP): Therapeutic Indication for Prostate Cancer. Biochem. $J$ 2011, 436, 481-91.

50. Mattsson, K.; Pokrovskaja, K.; Kiss, C.; Klein, G.; Szekely, L., Proteins Associated with the Promyelocytic Leukemia Gene Product (PML)-Containing Nuclear Body Move to the Nucleolus upon Inhibition of Proteasome-Dependent Protein Degradation. Proc Natl Acad Sci U $S$ A 2001, 98, 1012-7.

51. Ogiso, Y.; Tomida, A.; Tsuruo, T., Nuclear Localization of Proteasomes Participates in Stress-Inducible Resistance of Solid Tumor Cells to Topoisomerase II-Directed Drugs. Cancer Res 2002, 62, 5008-12.

52. Fujita, T.; Iwasa, J.; Hansch, C., A New Substituent Constant, $\pi$, Derived from Partition Coefficients. J. Am. Chem. Soc. 1964, 86, 5175-5180.

53. Hansch, C.; Maloney, P. P.; Fujita, T.; Muir, R. M., Correlation of Biological Activity of Phenoxyacetic Acids with Hammett Substituent Constants and Partition Coefficients. Nature 1962, 194, 178.

54. Hall, I. H.; Lackey, C. B.; Kistler, T. D.; Ives, J. S.; Beraldo, H.; Ackerman, L. J.; West, D. X., The Cytotoxicity of Symmetrical and Unsymmetrical Bis(thiosemicarbazones) and their Metal Complexes in Murine and Human Tumor Cells. Arch Pharm (Weinheim) 2000, 333, 21725.

55. Gopal, Y. N.; Jayaraju, D.; Kondapi, A. K., Inhibition of Topoisomerase II Catalytic Activity by Two Ruthenium Compounds: A Ligand-Dependent Mode of Action. Biochemistry 1999, 38, 4382-8.

56. Bhuyan, B. K.; Betz, T., Studies on the Mode of Action of the Copper(II) Chelate of 2keto-3-ethoxybutyraldehyde-bis(thiosemicarbazone). Cancer Res 1968, 28, 758-63.

57. Vock, C. A.; Ang, W. H.; Scolaro, C.; Phillips, A. D.; Lagopoulos, L.; Juillerat-Jeanneret, L.; Sava, G.; Scopelliti, R.; Dyson, P. J., Development of Ruthenium Antitumor Drugs that Overcome Multidrug Resistance Mechanisms. J. Med. Chem. 2007, 50, 2166-2175.

58. Paterson, B. M.; Donnelly, P. S., Copper Complexes of Bis(thiosemicarbazones): From Chemotherapeutics to Diagnostic and Therapeutic Radiopharmaceuticals. Chem. Soc. Rev. 2011, 40, 3005-18.

59. Miller, M. C., 3rd; Stineman, C. N.; Vance, J. R.; West, D. X.; Hall, I. H., The Cytotoxicity of Copper(II) Complexes of 2-acetyl-pyridyl-4N-substituted Thiosemicarbazones. Anticancer Res. 1998, 18, 4131-9. 
60. Miller, M. C.; Stineman, C. N.; Vance, J. R.; West, D. X.; Hall, I. H., Multiple Mechanisms for Cytotoxicity Induced by Copper(II) Complexes of 2-acetylpyrazine-Nsubstituted Thiosemicarbazones. Appl. Organomet. Chem. 1999, 13, 9-19.

61. Zeglis, B. M.; Divilov, V.; Lewis, J. S., Role of Metalation in the Topoisomerase II $\alpha$ Inhibition and Antiproliferation Activity of a Series of $\alpha$-Heterocyclic-N4-Substituted Thiosemicarbazones and their $\mathrm{Cu}(\mathrm{II})$ Complexes. J. Med. Chem. 2011, 54, 2391-8.

62. Tewey, K. M.; Rowe, T. C.; Yang, L.; Halligan, B. D.; Liu, L. F., Adriamycin-Induced DNA Damage Mediated by Mammalian DNA Topoisomerase II. Science 1984, 226, 466-8.

63. Palma, E.; Mendes, F.; Morais, G. R.; Rodrigues, I.; Santos, I. C.; Campello, M. P. C.; Raposinho, P.; Correia, I.; Gama, S.; Belo, D.; Alves, V.; Abrunhosa, A. J.; Santos, I.; Paulo, A., Biophysical Characterization and Antineoplastic Activity of New Bis(thiosemicarbazonato) $\mathrm{Cu}$ (II) Complexes. J. Inorg. Biochem. 2017, 167, 68-79.

64. Price, K. A.; Crouch, P. J.; Volitakis, I.; Paterson, B. M.; Lim, S.; Donnelly, P. S.; White, A. R., Mechanisms Controlling the Cellular Accumulation of Copper Bis(thiosemicarbazonato) Complexes. Inorg. Chem. 2011, 50, 9594-9605.

65. Acevedo, K. M.; Hayne, D. J.; McInnes, L. E.; Noor, A.; Duncan, C.; Moujalled, D.; Volitakis, I.; Rigopoulos, A.; Barnham, K. J.; Villemagne, V. L.; White, A. R.; Donnelly, P. S., Effect of Structural Modifications to Glyoxal-bis(thiosemicarbazonato)copper(II) Complexes on Cellular Copper Uptake, Copper-Mediated ATP7A Trafficking, and P-Glycoprotein Mediated Efflux. J. Med. Chem. 2018, 61, 711-723.

66. Stefani, C.; Al-Eisawi, Z.; Jansson, P. J.; Kalinowski, D. S.; Richardson, D. R., Identification of Differential Anti-Neoplastic Activity of Copper Bis(thiosemicarbazones) that is Mediated by Intracellular Reactive Oxygen Species Generation and Lysosomal Membrane Permeabilization. J. Inorg. Biochem. 2015, 152, 20-37.

67. Lovejoy, D. B.; Jansson, P. J.; Brunk, U. T.; Wong, J.; Ponka, P.; Richardson, D. R., Antitumor Activity of Metal-Chelating Compound Dp44mT is Mediated by Formation of a Redox-Active Copper Complex that Accumulates in Lysosomes. Cancer Res 2011, 71, 5871-80. 68. Brewer, G. J.; Dick, R. D.; Grover, D. K.; LeClaire, V.; Tseng, M.; Wicha, M.; Pienta, K.; Redman, B. G.; Jahan, T.; Sondak, V. K.; Strawderman, M.; LeCarpentier, G.; Merajver, S. D., Treatment of Metastatic Cancer with Tetrathiomolybdate, an Anticopper, Antiangiogenic Agent: Phase I Study. Clin Cancer Res 2000, 6, 1-10.

69. U.S. EIA, M. E. R., Table 1.3, April 2017, preliminary data.

70. Hu, X.; Brunschwig, B. S.; Peters, J. C., Electrocatalytic Hydrogen Evolution at Low Overpotentials by Cobalt Macrocyclic Glyoxime and Tetraimine Complexes. J. Am. Chem. Soc. 2007, 129, 8988-8998.

71. Savéant, J. M.; Su, K. B., Homogeneous Redox Catalysis of Electrochemical Reaction: Part VI. Zone Diagram Representation of the Kinetic Regimes. J. Electroanal. Chem. Interfacial Electrochem. 1984, 171, 341-349.

72. Marinescu, S. C.; Winkler, J. R.; Gray, H. B., Molecular Mechanisms of CobaltCatalyzed Hydrogen Evolution. Proc Natl Acad Sci U S A 2012, 109, 15127-31.

73. Kotani, H.; Hanazaki, R.; Ohkubo, K.; Yamada, Y.; Fukuzumi, S., Size- and ShapeDependent Activity of Metal Nanoparticles as Hydrogen-Evolution Catalysts: Mechanistic Insights into Photocatalytic Hydrogen Evolution. Chem. Eur. J. 2011, 17, 2777-2785.

74. Haddad, A. Z.; Kumar, D.; Ouch Sampson, K.; Matzner, A. M.; Mashuta, M. S.; Grapperhaus, C. A., Proposed Ligand-Centered Electrocatalytic Hydrogen Evolution and Hydrogen Oxidation at a Noninnocent Mononuclear Metal-Thiolate. J. Am. Chem. Soc. 2015, 137, 9238-9241.

75. Jain, R. Evaluating Ligand Assisted Activation of Small Molecules Through Proton Redcution and Alcohol Oxidation. University of Louisville, 2017. 
76. Jain, R.; Mamun, A. A.; Buchanan, R. M.; Kozlowski, P. M.; Grapperhaus, C. A., Ligand-Assisted Metal-Centered Electrocatalytic Hydrogen Evolution upon Reduction of a Bis(thiosemicarbazonato)Ni(II) Complex. Inorg. Chem. 2018, 57, 13486-13493.

77. Zhang, W. Heterogenization of Complexes with Redox Active Ligands for Hydrogen Evolution Reaction. University of Louisville, 2017.

78. Stoll, S.; Schweiger, A., EasySpin, a Comprehensive Software Package for Spectral Simulation and Analysis in EPR. J. Magn. Reson 2006, 178, 42-55.

79. Carta, F.; Akdemir, A.; Scozzafava, A.; Masini, E.; Supuran, C. T., Xanthates and Trithiocarbonates Strongly Inhibit Carbonic Anhydrases and Show Antiglaucoma Effects In Vivo. J. Med. Chem. 2013, 56, 4691-4700.

80. Reüfenacht, K., Arbetien über Phosphosäure- und Thiosphosphorsäureester mit einem Heterocyclischen Substituenten Thiadiazol-Ringschluss und eine Dabei Auftretende Methylübertragung. Helv. Chim. Acta 1972, 120, 1178-1187.

81. Bocokic, V.; Lutz, M.; Spek, A. L.; Reek, J. N. H., Bis-(thiosemicarbazonato) Zn(II) Complexes as Building Blocks for Construction of Supramolecular Catalysts. Dalton Trans. 2012, 41, 3740-3750.

82. Vishnosky, N. S.; Mashuta, M. S.; Buchanan, R. M.; Grapperhaus, C. A., Syntheses, structures, and electrochemical studies of N,N'-bis(alkylthiocarbamate)butane-2,3-diimine $\mathrm{Cu}$ (II) complexes as pendent alkoxy derivatives of Cu(ATSM). Inorg. Chim. Acta 2017, 461, 45-51.

83. Vishnosky, N. S.; Andres, S.; Mashuta, M. S.; Bates, P. J.; Buchanan, R. M.;

Grapperhaus, C. A., in preparation.

84. Vishnosky, N. S.; Gupta, A. J.; Mashuta, M. S.; Gupta, G.; Buchanan, R. M.; Grapperhaus, C. A., under revision.

85. CrysAlis PRO (CCD and RED), V 1.171.36.32; Oxford Diffraction Ltd.: Yarnton, England, 2013.

86. SCALE3, ABSPACK included in the CrysAlis PRO RED V 1.171.36.32.

87. Sheldrick, G., A Short History of SHELX. Acta Crystallogr. Sect. A: Found. Crystallogr. 2008, 64, 112-122.

88. Bates, P. J.; Kahlon, J. B.; Thomas, S. D.; Trent, J. O.; Miller, D. M., Antiproliferative Activity of G-rich Oligonucleotides Correlates with Protein Binding. J. Biol. Chem. 1999, 274, 26369-26377.

89. S Šalipur, F. R.; Merit Reyes-Reyes, E.; Xu, B.; Hammond, G. B.; Bates, P. J., A novel small molecule that induces oxidative stress and selectively kills malignant cells. Free Radical Biol. Med. 2014, 68, 110-121.

90. Morgan, D. M., Tetrazolium (MTT) Assay for Cellular Viability and Activity. Methods Mol Biol 1998, 79, 179-83.

91. Cowley, A. R.; Dilworth, J. R.; Donnelly, P. S.; Labisbal, E.; Sousa, A., An Unusual Dimeric Structure of a $\mathrm{Cu}(\mathrm{I}) \mathrm{Bis}$ (thiosemicarbazone) Complex: Implications for the Mechanism of Hypoxic Selectivity of the Cu(II) Derivatives. J. Am. Chem. Soc. 2002, 124, 5270-5271. 92. Christlieb, M.; Dilworth, J. R., Ligands for Molecular Imaging: The Synthesis of Bis(thiosemicarbazone) Ligands. Chem. - Eur. J. 2006, 12, 6194-6206.

93. Ballschmitter, K.; Greber, H.; Steinhäuser, H., Substituentenffekte bei LigandMatallelektronenübergängen von $\mathrm{Cu}$ (II)-Chelaten der $\alpha$-diketo-bis-thiosemicarbazone. J. Inorg. Nucl. Chem. 1978, 40, 631-634.

94. McQuade, P.; Martin, K. E.; Castle, T. C.; Went, M. J.; Blower, P. J.; Welch, M. J.; Lewis, J. S., Investigation Into ${ }^{64} \mathrm{Cu}$-labeled Bis(selenosemicarbazone) and Bis(thiosemicarbazone) Complexes as Hypoxia Imaging Agents. Nucl. Med. Biol. 2005, 32, $147-$ 156.

95. Farrugia, L., ORTEP-3 for Windows - a Version of ORTEP-III with a Graphical User Interface (GUI). J. Appl. Crystallogr. 1997, 30, 565. 
96. Blower, P. J.; Castle, T. C.; Cowley, A. R.; Dilworth, J. R.; Donnelly, P. S.; Labisbal, E.; Sowrey, F. E.; Teat, S. J.; Went, M. J., Structural Trends in Copper(II) Bis(thiosemicarbazone) Radiopharmaceuticals. Dalton Trans. 2003, 4416-4425.

97. Hoffert, M. I.; Caldeira, K.; Jain, A. K.; Haites, E. F.; Harvey, L. D. D.; Potter, S. D.; Schlesinger, M. E.; Schneider, S. H.; Watts, R. G.; Wigley, T. M. L.; Wuebbles, D. J., Energy Implications of Future Stabilization of Atmospheric $\mathrm{CO}_{2}$ Content. Nature 1998, 395, 881-884. 98. Lewis, N. S.; Nocera, D. G., Powering the Planet: Chemical Challenges in Solar Energy Utilization. Proc. Natl. Acad. Sci. U. S. A. 2006, 103, 15729-15735.

99. Park, J. H.; Kim, S.; Bard, A. J., Novel Carbon-Doped $\mathrm{TiO}_{2} \mathrm{Nanotube}$ Arrays with High Aspect Ratios for Efficient Solar Water Splitting. Nano Lett. 2006, 6, 24-28.

100. Khaselev, O.; Turner, J. A., A Monolithic Photovoltaic-Photoelectrochemical Device for Hydrogen Production via Water Splitting. Science 1998, 280, 425-427.

101. Kim, T. W.; Choi, K.-S., Nanoporous $\mathrm{BiVO}_{4}$ Photoanaodes with Dual-Layer Oxygen Evolution Catalysts for Solar Water Splitting. Science 2014, 343, 990-994.

102. Sivula, K.; Le Formal, F.; Grätzel, M., Solar Water Splitting: Progress Using Hematite $\left(\alpha-\mathrm{Fe}_{2} \mathrm{O}_{3}\right)$ Photoelectrodes. ChemSusChem 2011, 4, 432-449.

103. Turner, J. A., A Realizable Renewable Energy Future. Science 1999, 285, 687-689.

104. Council, N. R., The hydrogen economy: opportunities, costs, barriers, and R\&D needs. National Academies Press: 2004.

105. Kibler, L. A., Hydrogen Electrocatalysis. ChemPhysChem 2006, 7, 985-991.

106. Subbaraman, R.; Tripkovic, D.; Strmcnik, D.; Chang, K.-C.; Uchimura, M.; Paulikas, A. P.; Stamenkovic, V.; Markovic, N. M., Enhancing Hydrogen Evolution Activity in Water Splitting by Tailoring $\mathrm{Li}^{+}-\mathrm{Ni}(\mathrm{OH})_{2}-\mathrm{Pt}$ Interfaces. Science 2011, 334, 1256-1260.

107. Di Giovanni, C.; Wang, W.-A.; Nowak, S.; Grenèche, J.-M.; Lecoq, H.; Mouton, L.; Giraud, M.; Tard, C., Bioinspired Iron Sulfide Nanoparticles for Cheap and Long-Lived Electrocatalytic Molecular Hydrogen Evolution in Neutral Water. ACS Catal. 2014, 4, 681-687. 108. Popczun, E. J.; Read, C. G.; Roske, C. W.; Lewis, N. S.; Schaak, R. E., Highly Active Electrocatalysis of the Hydrogen Evolution Reaction by Cobalt Phosphide Nanoparticles. Angew. Chem. Int. Ed. 2014, 53, 5427-5430.

109. Lei, H.; Han, A.; Li, F.; Zhang, M.; Han, Y.; Du, P.; Lai, W.; Cao, R., Electrochemical, Spectroscopic and Theoretical Studies of a Simple Bifunctional Cobalt Corrole Catalyst for Oxygen Evolution and Hydrogen Production. PCCP 2014, 16, 1883-1893.

110. Mondal, B.; Sengupta, K.; Rana, A.; Mahammed, A.; Botoshansky, M.; Dey, S. G.; Gross, Z.; Dey, A., Cobalt Corrole Catalyst for Efficient Hydrogen Evolution Reaction from $\mathrm{H}_{2} \mathrm{O}$ under Ambient Conditions: Reactivity, Spectroscopy, and Density Functional Theory Calculations. Inorg. Chem. 2013, 52, 3381-3387.

111. Tang, C. P., Z.; Liu, Q.; Asiri, A. M.; Sun, X., NiS 2 Nanosheets Array Grown on Carbon Cloth as an Efficient 3D Hydrogen Evolution Cathode. Electrochim. Acta 2015, 153, 508-514.

112. Tran, P. D.; Le Goff, A.; Heidkamp, J.; Jousselme, B.; Guillet, N.; Palacin, S.; Dau, H.; Fontecave, M.; Artero, V., Noncovalent Modification of Carbon Nanotubes with PyreneFunctionalized Nickel Complexes: Carbon Monoxide Tolerant Catalysts for Hydrogen Evolution and Uptake. Angew. Chem. 2011, 123, 1407-1410.

113. Rodríguez-Maciá, P.; Priyadarshani, N.; Dutta, A.; Weidenthaler, C.; Lubitz, W.; Shaw, W. J.; Rüdiger, O., Covalent Attachment of the Water-insoluble Ni(PCy2NPhe2)2 Electrocatalyst to Electrodes Showing Reversible Catalysis in Aqueous Solution. Electroanalysis 2016, 28, 2452-2458.

114. Oughli, A. A.; Ruff, A.; Boralugodage, N. P.; Rodríguez-Maciá, P.; Plumeré, N.; Lubitz, W.; Shaw, W. J.; Schuhmann, W.; Rüdiger, O., Dual Properties of a Hydrogen Oxidation NiCatalyst Entrapped Within a Polymer Promote Self-Defense Against Oxygen. Nat. Commun. 2018, 9, 864-869. 
115. Tian, J.; Liu, Q.; Cheng, N.; Asiri, A. M.; Sun, X., Self-Supported Cu 3 P Nanowire Arrays as an Integrated High-Performance Three-Dimensional Cathode for Generating Hydrogen from Water. Angew. Chem. Int. Ed. 2014, 53, 9577-9581.

116. Ye, G.; Gong, Y.; Lin, J.; Li, B.; He, Y.; Pantelides, S. T.; Zhou, W.; Vajtai, R.; Ajayan, P. M., Defects Engineered Monolayer $\mathrm{MoS}_{2}$ for Improved Hydrogen Evolution Reaction. Nano Lett. 2016, 16, 1097-1103.

117. Li, Y.; Wang, H.; Xie, L.; Liang, Y.; Hong, G.; Dai, H., $\mathrm{MoS}_{2}$ Nanoparticles Grown on Graphene: An Advanced Catalyst for the Hydrogen Evolution Reaction. J. Am. Chem. Soc. 2011, 133, 7296-7299.

118. Yan, Y.; Xia, B.; Xu, Z.; Wang, X., Recent Development of Molybdenum Sulfides as Advanced Electrocatalysts for Hydrogen Evolution Reaction. ACS Catal. 2014, 4, 1693-1705.

119. Popczun, E. J.; McKone, J. R.; Read, C. G.; Biacchi, A. J.; Wiltrout, A. M.; Lewis, N. S.; Schaak, R. E., Nanostructured Nickel Phosphide as an Electrocatalyst for the Hydrogen Evolution Reaction. J. Am. Chem. Soc. 2013, 135, 9267-9270.

120. Kong, D.; Cha, J. J.; Wang, H.; Lee, H. R.; Cui, Y., First-Row Transition Metal Dichalcogenide Catalysts for Hydrogen Evolution Reaction. Energy Environ. Sci. 2013, 6, 3553 3558 .

121. Bonde, J.; Moses, P. G.; Jaramillo, T. F.; Nørskov, J. K.; Chorkendorff, I., Hydrogen Evolution on Nano-Particulate Transition Metal Sulfides. Faraday Discuss. 2009, 140, 219-231. 122. Merki, D.; Hu, X., Recent Developments of Molybdenum and Tungsten Sulfides as Hydrogen Evolution Catalysts. Energy Environ. Sci. 2011, 4, 3878-3888.

123. Rountree, E. S.; Dempsey, J. L., Reactivity of Proton Sources with a Nickel Hydride Complex in Acetonitrile: Implications for the Study of Fuel-Forming Catalysts. Inorg. Chem. 2016, 55, 5079-5087.

124. Dempsey, J. L.; Brunschwig, B. S.; Winkler, J. R.; Gray, H. B., Hydrogen Evolution Catalyzed by Cobaloximes. Acc. Chem. Res. 2009, 42, 1995-2004.

125. Mejia-Rodriguez, R.; Chong, D.; Reibenspies, J. H.; Soriaga, M. P.; Darensbourg, M. Y., The Hydrophilic Phosphatriazaadamantane Ligand in the Development of $\mathrm{H}_{2}$ Production Electrocatalysts: Iron Hydrogenase Model Complexes. J. Am. Chem. Soc. 2004, 126, 1200412014.

126. Merki, D.; Vrubel, H.; Rovelli, L.; Fierro, S.; Hu, X., Fe, Co, and Ni Ions Promote the Catalytic Activity of Amorphous Molybdenum Sulfide Films for Hydrogen Evolution. Chem. Sci. 2012, 3, 2515-2525.

127. Xiao, P.; Sk, M. A.; Thia, L.; Ge, X.; Lim, R. J.; Wang, J.-Y.; Lim, K. H.; Wang, X., Molybdenum Phosphide as an Efficient Electrocatalyst for the Hydrogen Evolution Reaction. Energy Environ. Sci. 2014, 7, 2624-2629.

128. Jaramillo, T. F.; Jørgensen, K. P.; Bonde, J.; Nielsen, J. H.; Horch, S.; Chorkendorff, I., Identification of Active Edge Sites for Electrochemical $\mathrm{H}_{2}$ Evolution from $\mathrm{MoS}_{2}$ Nanocatalysts. Science 2007, 317, 100-102.

129. Lazarides, T.; McCormick, T.; Du, P.; Luo, G.; Lindley, B.; Eisenberg, R., Making Hydrogen from Water Using a Homogeneous System Without Noble Metals. J. Am. Chem. Soc. 2009, 131, 9192-9194.

130. Fukuzumi, S.; Yamada, Y.; Suenobu, T.; Ohkubo, K.; Kotani, H., Catalytic Mechanisms of Hydrogen Evolution with Homogeneous and Heterogeneous Catalysts. Energy Environ. Sci. 2011, 4, 2754-2766.

131. Ford, P. C., The Water Gas Shift Reaction: Homogeneous Catalysis by Ruthenium and Other Metal Carbonyls. Acc. Chem. Res. 1981, 14, 31-37.

132. Rakowski DuBois, M.; DuBois, D. L., Development of Molecular Electrocatalysts for $\mathrm{CO}_{2}$ Reduction and $\mathrm{H}_{2}$ Production/Oxidation. Acc. Chem. Res. 2009, 42, 1974-1982.

133. Wiese, S.; Kilgore, U. J.; DuBois, D. L.; Bullock, R. M., [Ni( $\left.\left.\mathrm{PMe}_{2} \mathrm{NPh}_{2}\right)_{2}\right]\left(\mathrm{BF}_{4}\right)_{2}$ as an Electrocatalyst for $\mathrm{H}_{2}$ Production. ACS Catal. 2012, 2, 720-727. 
134. Le Goff, A.; Artero, V.; Jousselme, B.; Tran, P. D.; Guillet, N.; Métayé, R.; Fihri, A.; Palacin, S.; Fontecave, M., From Hydrogenases to Noble Metal-Free Catalytic Nanomaterials for $\mathrm{H}_{2}$ Production and Uptake. Science 2009, 326, 1384-1387.

135. Pantani, O.; Anxolabéhère-Mallart, E.; Aukauloo, A.; Millet, P., Electroactivity of Cobalt and Nickel Glyoximes with Regard to the Electro-Reduction of Protons into Molecular Hydrogen in Acidic Media. Electrochem. Commun. 2007, 9, 54-58.

136. Berben, L. A.; Peters, J. C., Hydrogen Evolution by Cobalt Tetraimine Catalysts Adsorbed on Electrode Surfaces. Chem. Commun. 2010, 46, 398-400.

137. Zhang, W.; Haddad, A. Z.; Garabato, B. D.; Kozlowski, P. M.; Buchanan, R. M.; Grapperhaus, C. A., Translation of Ligand-Centered Hydrogen Evolution Reaction Activity and Mechanism of a Rhenium-Thiolate from Solution to Modified Electrodes: A Combined Experimental and Density Functional Theory Study. Inorg. Chem. 2017, 56, 2177-2187. 138. Abe, T.; Taguchi, F.; Imaya, H.; Zhao, F.; Zhang, J.; Kaneko, M., Highly Active Electrocatalysis by Cobalt Tetraphenylporphyrin Incorporated in a Nafion Membrane for Proton Reduction. Polym. Adv. Technol. 1998, 9, 559-562.

139. Koca, A., Copper Phthalocyanine Complex as Electrocatalyst for Hydrogen Evolution Reaction. Electrochem. Commun. 2009, 11, 838-841.

140. Koca, A.; Kalkan, A.; Altuntaş Bayır, Z., Electrochemical, In Situ Spectroelectrochemical, In Situ Electrocolorimetric and Electrocatalytic Characterization of Metallophthalocyanines Bearing Four Dioctylaminocarbonyl Biphenyloxy Substituents. Electroanalysis 2010, 22, 310-319.

141. Koca, A.; Kalkan, A.; Bayır, Z. A., Electrocatalytic Oxygen Reduction and Hydrogen Evolution Reactions on Phthalocyanine Modified Electrodes: Electrochemical, In Situ Spectroelectrochemical, and In Situ Electrocolorimetric Monitoring. Electrochim. Acta 2011, 56, 5513-5525.

142. Koca, A., Hydrogen Evolution Reaction on Glassy Carbon Electrode Modified with Titanyl Phthalocyanines. Int. J. Hydrogen Energy 2009, 34, 2107-2112.

143. Grass, V.; Lexa, D.; Savéant, J.-M., Electrochemical Generation of Rhodium Porphyrin Hydrides. Catalysis of Hydrogen Evolution. J. Am. Chem. Soc. 1997, 119, 7526-7532.

144. Jurss, J. W.; Khnayzer, R. S.; Panetier, J. A.; El Roz, K. A.; Nichols, E. M.; HeadGordon, M.; Long, J. R.; Castellano, F. N.; Chang, C. J., Bioinspired Design of Redox-Active Ligands for Multielectron Catalysis: Effects of Positioning Pyrazine Reservoirs on Cobalt for Electro- and Photocatalytic Generation of Hydrogen from Water. Chem. Sci. 2015, 6, 4954-4972. 145. Thompson, E. J.; Berben, L. A., Electrocatalytic Hydrogen Production by an Aluminum(III) Complex: Ligand-Based Proton and Electron Transfer. Angew. Chem. Int. Ed. 2015, 54, 11642-11646.

146. Sherbow, T. J.; Fettinger, J. C.; Berben, L. A., Control of Ligand pKa Values Tunes the Electrocatalytic Dihydrogen Evolution Mechanism in a Redox-Active Aluminum(III) Complex. Inorg. Chem. 2017, 56, 8651-8660.

147. Panetier, J. A.; Letko, C. S.; Tilley, T. D.; Head-Gordon, M., Computational Characterization of Redox Non-Innocence in Cobalt-Bis(Diaryldithiolene)-Catalyzed Proton Reduction. J. Chem. Theory Comput. 2016, 12, 223-230.

148. Walter, M. G.; Warren, E. L.; McKone, J. R.; Boettcher, S. W.; Mi, Q.; Santori, E. A.; Lewis, N. S., Solar Water Splitting Cells. Chem. Rev. 2010, 110, 6446-6473.

149. Weber, M. F.; Dignam, M. J., Efficiency of Splitting Water with Semiconducting Photoelectrodes. J. Electrochem. Soc. 1984, 131, 1258-1265.

150. Conway, B. E.; Tilak, B. V., Interfacial Processes Involving Electrocatalytic Evolution and Oxidation of $\mathrm{H}_{2}$, and the Role of Chemisorbed H. Electrochim. Acta 2002, 47, 3571-3594. 151. Fletcher, S., Tafel Slopes from First Principles. J. Solid State Electrochem. 2009, 13, 537-549. 
152. Shinagawa, T.; Garcia-Esparza, A. T.; Takanabe, K., Insight on Tafel Slopes from a Microkinetic Analysis of Aqueous Electrocatalysis for Energy Conversion. Sci. Rep. 2015, 5 , 13801-13821.

153. Sano, T.; Sasako, M.; Yamamoto, S.; Nashimoto, A.; Kurita, A.; Hiratsuka, M.; Tsujinaka, T.; Kinoshita, T.; Arai, K.; Yamamura, Y.; Okajima, K., Gastric cancer surgery: morbidity and mortality results from a prospective randomized controlled trial comparing D2 and extended para-aortic lymphadenectomy--Japan Clinical Oncology Group study 9501. J Clin Oncol 2004, 22, 2767-2773.

154. Heald, R. J.; Husband, E. M.; Ryall, R. D. H., The mesorectum in rectal cancer surgerythe clue to pelvic recurrence? Br. J. Surg. 1982, 69, 613-616.

155. Poleshuck, E. L.; Katz, J.; Andrus, C. H.; Hogan, L. A.; Jung, B. F.; Kulick, D. I.; Dworkin, R. H., Risk Factors for Chronic Pain Following Breast Cancer Surgery: A Prospective Study. J. Pain 2006, 7, 626-634.

156. Veronesi, U.; Valagussa, P., Inefficacy of internal mammary nodes dissection in breast cancer surgery. Cancer 1981, 47, 170-175.

157. Mazzaferri, E. L.; Jhiang, S. M., Long-Term Impact of Initial Surgical and Medical Therapy on Papillary and Follicular Thyroid Cancer. Am. J. Med. 1994, 97, 418-428.

158. Byar, D. P.; Corle, D. K., Hormone Therapy for Prostate Cancer: Results of the Veterans Administration Cooperative Urological Research Group Studies. J. Natl. Cancer Inst. Monogr. 1988, $165-170$.

159. Grodstein, F.; Newcomb, P. A.; Stampfer, M. J., Postmenopausal Hormone Therapy and the Risk of Colorectal Cancer: A Review and Meta-Analysis. Am. J. Med. 1999, 106, 574-582. 160. Leitzel, K.; Teramoto, Y.; Konrad, K.; Chinchilli, V. M.; Volas, G.; Grossberg, H.; Harvey, H.; Demers, L.; Lipton, A., Elevated Serum c-erbB-2 Antigen Levels and Decreased Response to Hormone Therapy of Breast Cancer. J. Clin. Oncol. 1995, 13, 1129-1135.

161. Pollack, A.; Zagars, G. K.; Starkschall, G.; Antolak, J. A.; Lee, J. J.; Huang, E.; von Eschenbach, A. C.; Kuban, D. A.; Rosen, I., Prostate Cancer Radiation Dose Response: Results of the M. D. Anderson Phase III Randomized Trial. Int. J. Radiat. Oncol., Biol., Phys. 2002, 53, 1097-1105.

162. Neugut, A. I.; Murray, T.; Santos, J.; Amols, H.; Hayes, M. K.; Flannery, J. T.; Robinson, E., Increased Risk of Lung Cancer After Breast Cancer Radiation Therapy in Cigarette Smokers. Cancer 1994, 73, 1615-1620.

163. Komaki, R.; Scott, C.; Ettinger, D.; Lee, J. S.; Fossella, F. V.; Curran, W.; Evans, R. F.; Rubin, P.; Byhardt, R. W., Randomized Study of Chemotherapy/Radiation Therapy Combinations for Favorable Patients With Locally Advanced Inoperable Nonsmall Cell Lung Cancer: Radiation Therapy Oncology Group (RTOG) 92-04. Int. J. Radiat. Oncol., Biol., Phys. 1997, 38, 149-155.

164. Sause, W.; Kolesar, P.; Taylor, S.; Johnson, D.; Livingston, R.; Komaki, R.; Emami, B.; Curran, W.; Byhardt, R.; Dar, A. R.; Turrisi, A., Final Results of Phase III Trial in Regionally Advanced Unresectable Non-Small Cell Lung Cancer: Radiation Therapy Oncology Group, Eastern Cooperative Oncology Group, and Southwest Oncology Group. Chest 2000, 117, 358364.

165. Blattman, J. N.; Greenberg, P. D., Cancer Immunotherapy: A Treatment for the Masses. Science 2004, 305, 200-205.

166. Schuler, G.; Schuler-Thurner, B.; Steinman, R. M., The Use of Dendritic Cells in Cancer Immunotherapy. Curr. Opin. Immunol. 2003, 15, 138-147.

167. Hoos, A.; Eggermont, A. M. M.; Janetzki, S.; Hodi, F. S.; Ibrahim, R.; Anderson, A.; Humphrey, R.; Blumenstein, B.; Old, L.; Wolchok, J., Improved Endpoints for Cancer Immunotherapy Trials. JNCI, J. Natl. Cancer Inst 2010, 102, 1388-1397. 
168. Sharma, P.; Wagner, K.; Wolchok, J. D.; Allison, J. P., Novel Cancer Immunotherapy Agents With Survival Benefit: Recent Successes and Next Steps. Nat. Rev. Cancer 2011, 11, 805812.

169. Schumacher, T. N.; Schreiber, R. D., Neoantigens in Cancer Immunotherapy. Science 2015, 348, 69-74.

170. Kelland, L., The resurgence of platinum-based cancer chemotherapy. Nat. Rev. Cancer 2007, 7, 573-584.

171. Kaufmann, S. H.; Earnshaw, W. C., Induction of Apoptosis by Cancer Chemotherapy. Experimental Cell Research 2000, 256, 42-49.

172. Farokhzad, O. C.; Cheng, J.; Teply, B. A.; Sherifi, I.; Jon, S.; Kantoff, P. W.; Richie, J. P.; Langer, R., Targeted Nanoparticle-Aptamer Bioconjugates for Cancer Chemotherapy in vivo.

Proc. Natl. Acad. Sci. U. S. A. 2006, 103, 6315-6320.

173. Frei, E., III; Canellos, G. P., Dose: A Critical Factor in Cancer Chemotherapy. Am. J. Med. 1980, 69, 585-594.

174. Scott, A. M.; Wolchok, J. D.; Old, L. J., Antibody Therapy of Cancer. Nat. Rev. Cancer 2012, 12, 278-287.

175. Adams, G. P.; Weiner, L. M., Monoclonal antibody therapy of cancer. Nat. Biotechnol. 2005, 23, 1147-1157.

176. Riethmüller, G.; Holz, E.; Schlimok, G.; Schmiegel, W.; Raab, R.; Höffken, K.; Gruber, R.; Funke, I.; Pichlmaier, H.; Hirche, H.; Buggisch, P.; Witte, J.; Pichlmayr, R., Monoclonal Antibody Therapy for Resected Dukes' C Colorectal Cancer: Seven-Year Outcome of a Multicenter Randomized Trial. J. Clin. Oncol. 1998, 16, 1788-1794.

177. Trikha, M.; Corringham, R.; Klein, B.; Rossi, J.-F., Targeted Anti-Interleukin-6 Monoclonal Antibody Therapy for Cancer: A Review of the Rationale and Clinical Evidence. Clin. Cancer Res. 2003, 9, 4653-4665.

178. Davis, T. A.; Grillo-López, A. J.; White, C. A.; McLaughlin, P.; Czuczman, M. S.; Link, B. K.; Maloney, D. G.; Weaver, R. L.; Rosenberg, J.; Levy, R., Rituximab Anti-CD20 Monoclonal Antibody Therapy in Non-Hodgkin's Lymphoma: Safety and Efficacy of ReTreatment. J. Clin. Oncol. 2000, 18, 3135-3143.

179. Johnson, D., ZView: a Software Program for IES Analysis, Version 2.8, Scribner Associates. Inc., Southern Pines, NC 2002, 200.

180. Chinarro, E.; Jurado, J.; Figueiredo, F.; Frade, J., Bulk and grain boundary conductivity of Ca0. 97Ti1- xFexO3- $\delta$ materials. Solid State Ionics 2003, 160, 161-168.

181. Gore, C. M.; White, J. O.; Wachsman, E. D.; Thangadurai, V., Effect of Composition and Microstructure on Electrical Properties and $\mathrm{CO}_{2}$ Stability of Donor-Doped, Proton Conducting $\mathrm{BaCe}_{1-(\mathrm{x}+\mathrm{y})} \mathrm{Zr}_{\mathrm{x}} \mathrm{Nb}_{\mathrm{y}} \mathrm{O}_{3}$. J. Mater. Chem. A 2014, 2, 2363-2373.

182. Li, Q.; Thangadurai, V., Synthesis, Structure and Electrical Properties of Mo-Doped $\mathrm{CeO}_{2}-$ Materials for SOFCs. Fuel Cells 2009, 9, 684-698.

183. Li, Q.; Thangadurai, V., A Comparative 2 and 4-Probe DC and 2-Probe AC Electrical Conductivity of Novel Co-Doped $\mathrm{Ce}_{0.9-\mathrm{x}} \mathrm{RE}_{\mathrm{x}} \mathrm{Mo}_{0.1} \mathrm{O}_{2.1-0.5 \mathrm{x}}(\mathrm{RE}=\mathrm{Y}, \mathrm{Sm}, \mathrm{Gd} ; \mathrm{x}=0.2,0.3) . J$. Mater. Chem. 2010, 20, 7970-7983.

184. Hjalmarsson, P.; Søgaard, M.; Mogensen, M., Electrochemical Behaviour of $\left(\mathrm{La}_{1-\mathrm{x}} \mathrm{Sr}_{\mathrm{x}}\right) \mathrm{sCo}_{1-\mathrm{y}} \mathrm{NiyO}_{3-\delta}$ as Porous SOFC Cathodes. Solid State Ionics 2009, 180, 1395-1405. 
APPENDIX

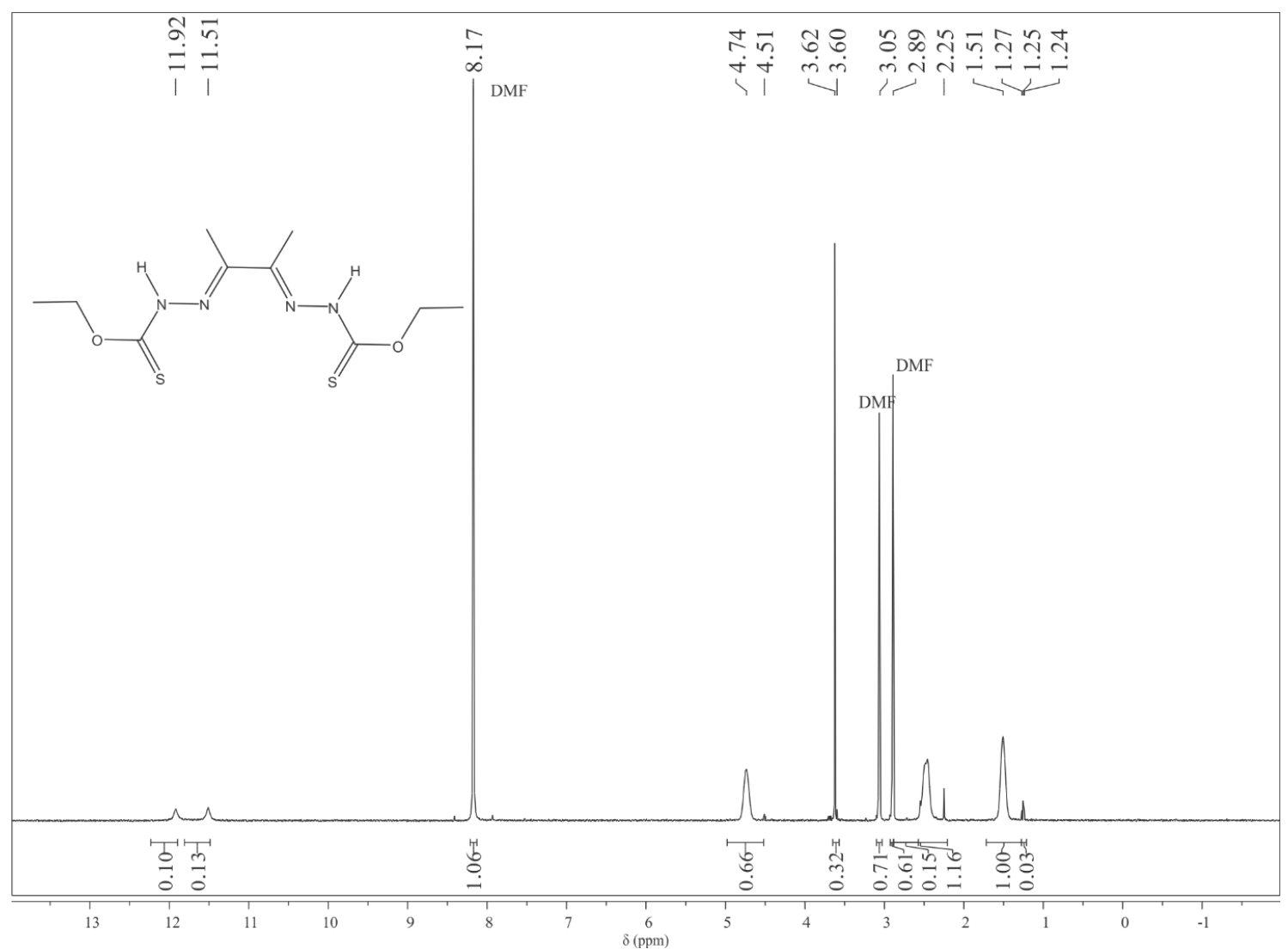

Figure A1. $\mathrm{H}_{2} \mathrm{ETCB}(5){ }^{1} \mathrm{H} \mathrm{NMR}$

$\left(\mathrm{C}_{2} \mathrm{D}_{6} \mathrm{NO}, 400 \mathrm{MHz}\right) \delta 1.51(\mathrm{br}, 6 \mathrm{H}) 2.46(\mathrm{br}, 4 \mathrm{H}) 4.74(\mathrm{~s}, 6 \mathrm{H}) 11.51(\mathrm{~s}, 1 \mathrm{H}) 11.92(\mathrm{~s}$, $1 \mathrm{H})$. 


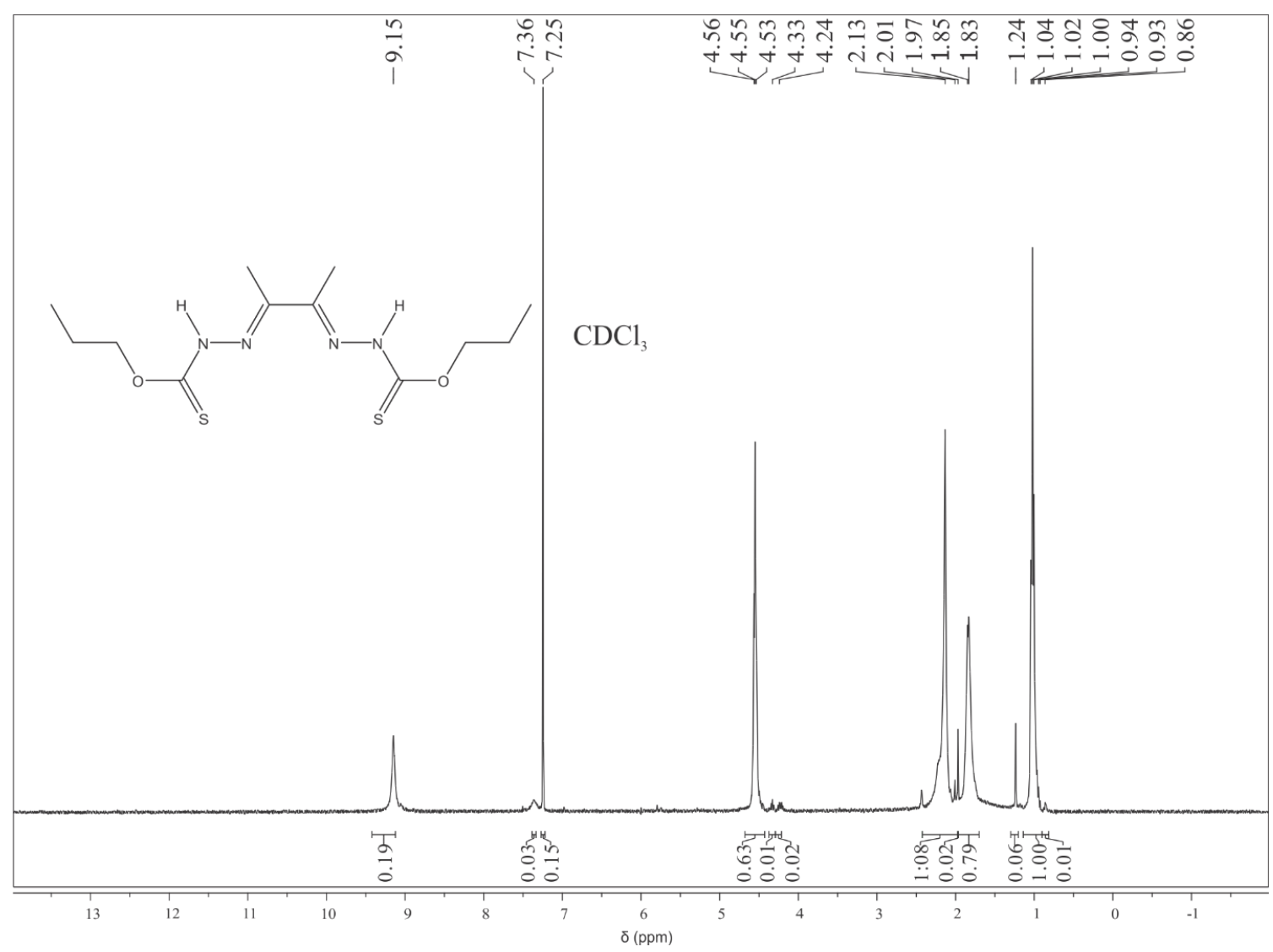

Figure A2. $\mathrm{H}_{2} \mathrm{PTCB}(6){ }^{1} \mathrm{H}$ NMR

$\left(\mathrm{CDCl}_{3}, 400 \mathrm{MHz}\right) \delta 0.99(\mathrm{t}, 6 \mathrm{H}, J=7.28) 1.84$ (quin, 4H, J=6.24) $2.13(\mathrm{~s}, 6 \mathrm{H}) 4.55$ (t, $4 \mathrm{H}, \mathrm{J}=6.22) 9.15(\mathrm{~s}, 2 \mathrm{H})$. 


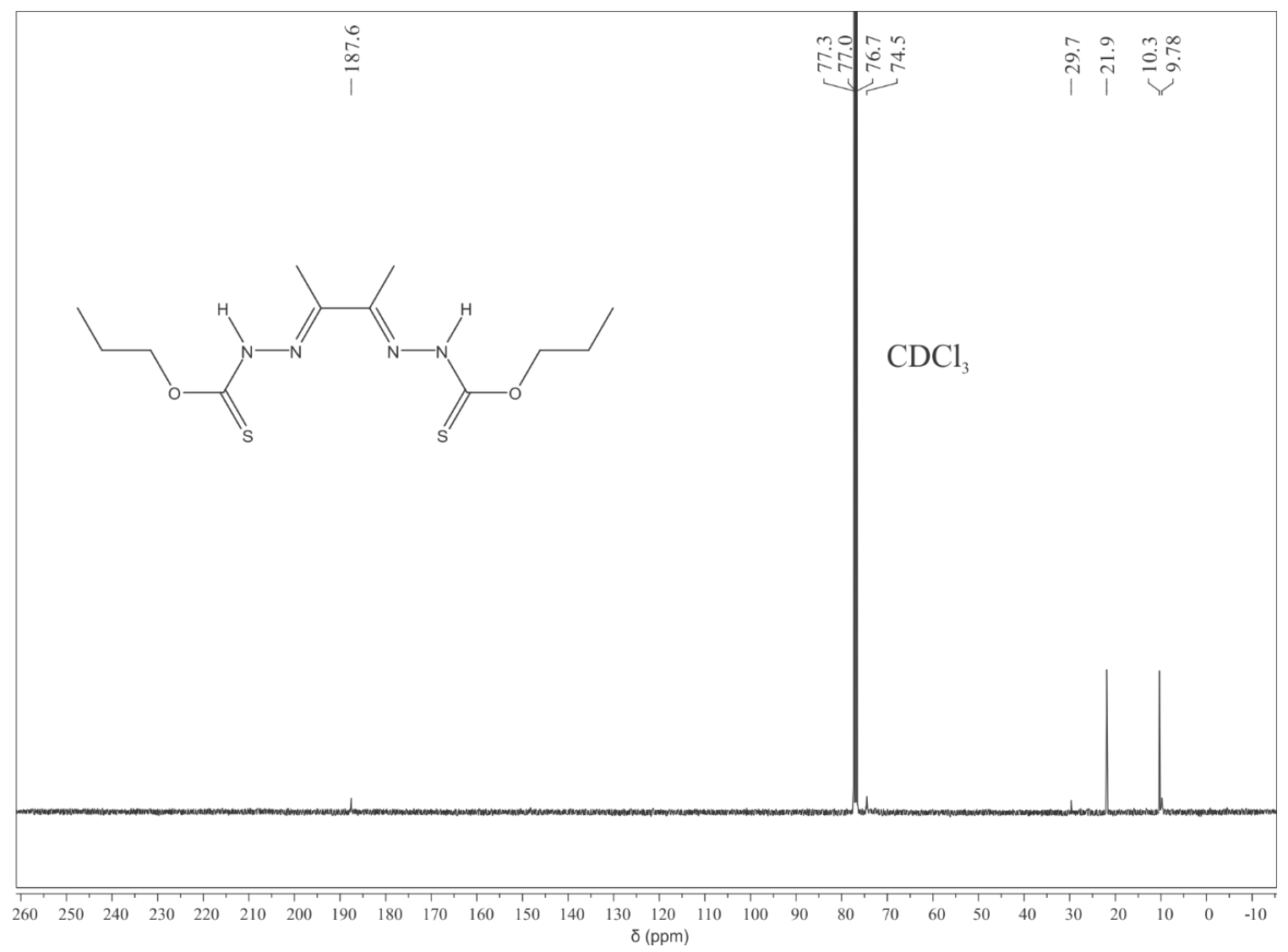

Figure A3. $\mathrm{H}_{2} \mathrm{PTCB}(6){ }^{13} \mathrm{C} \mathrm{NMR}$

$\left(\mathrm{CDCl}_{3}, 100 \mathrm{MHz}\right) \delta 9.78,10.3,21.9,29.7,187.6$ 


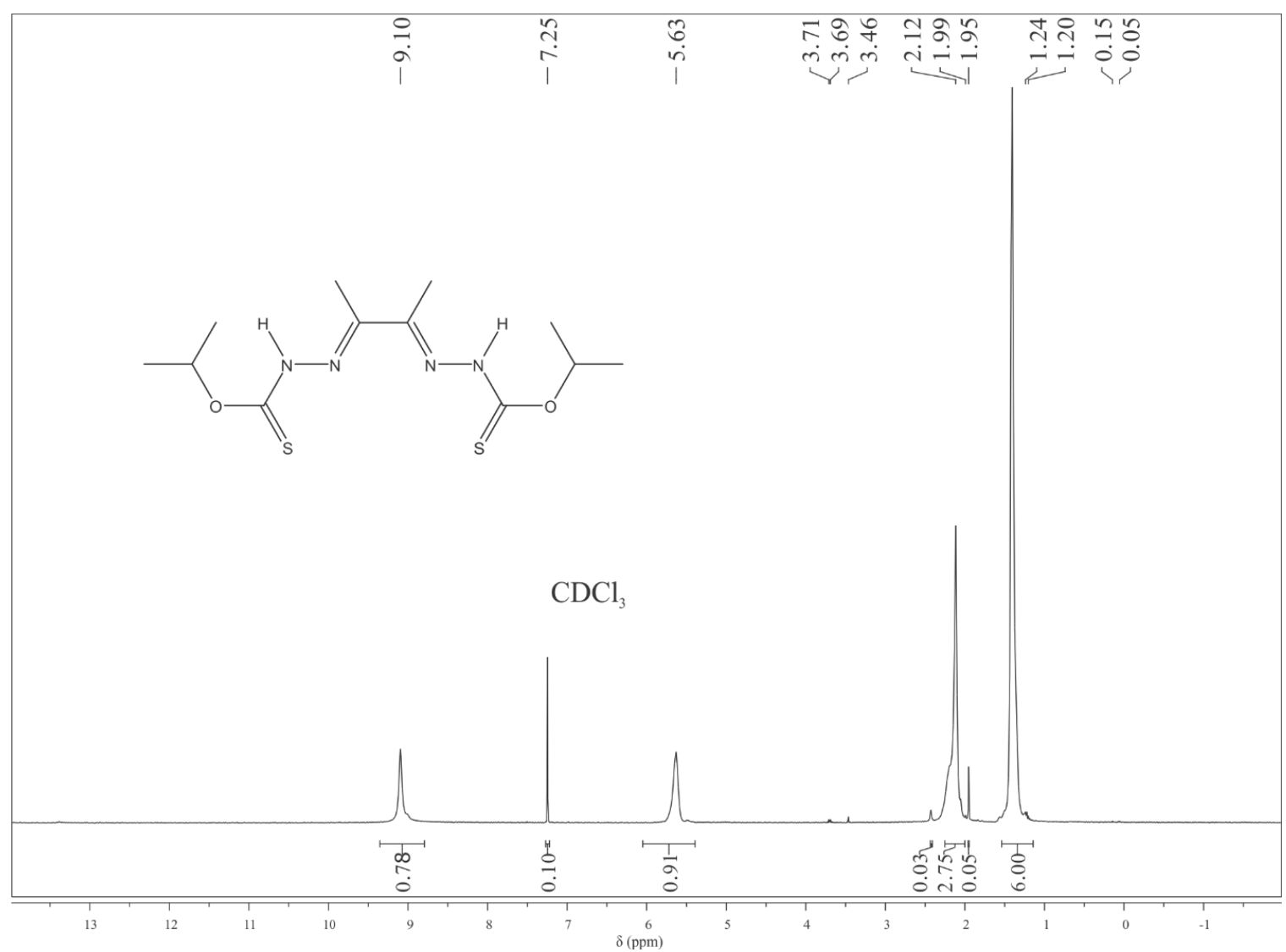

Figure A4. $\mathrm{H}_{2}{ }^{i} \mathrm{PTCB}(7){ }^{1} \mathrm{H}$ NMR

$\left(\mathrm{CDCl}_{3}, 400 \mathrm{MHz}\right) \delta 1.41(\mathrm{br}, 12 \mathrm{H}) 2.12(\mathrm{~s}, 6 \mathrm{H}) 5.63(\mathrm{br}, 2 \mathrm{H}) 9.10(\mathrm{~s}, 2 \mathrm{H})$. 


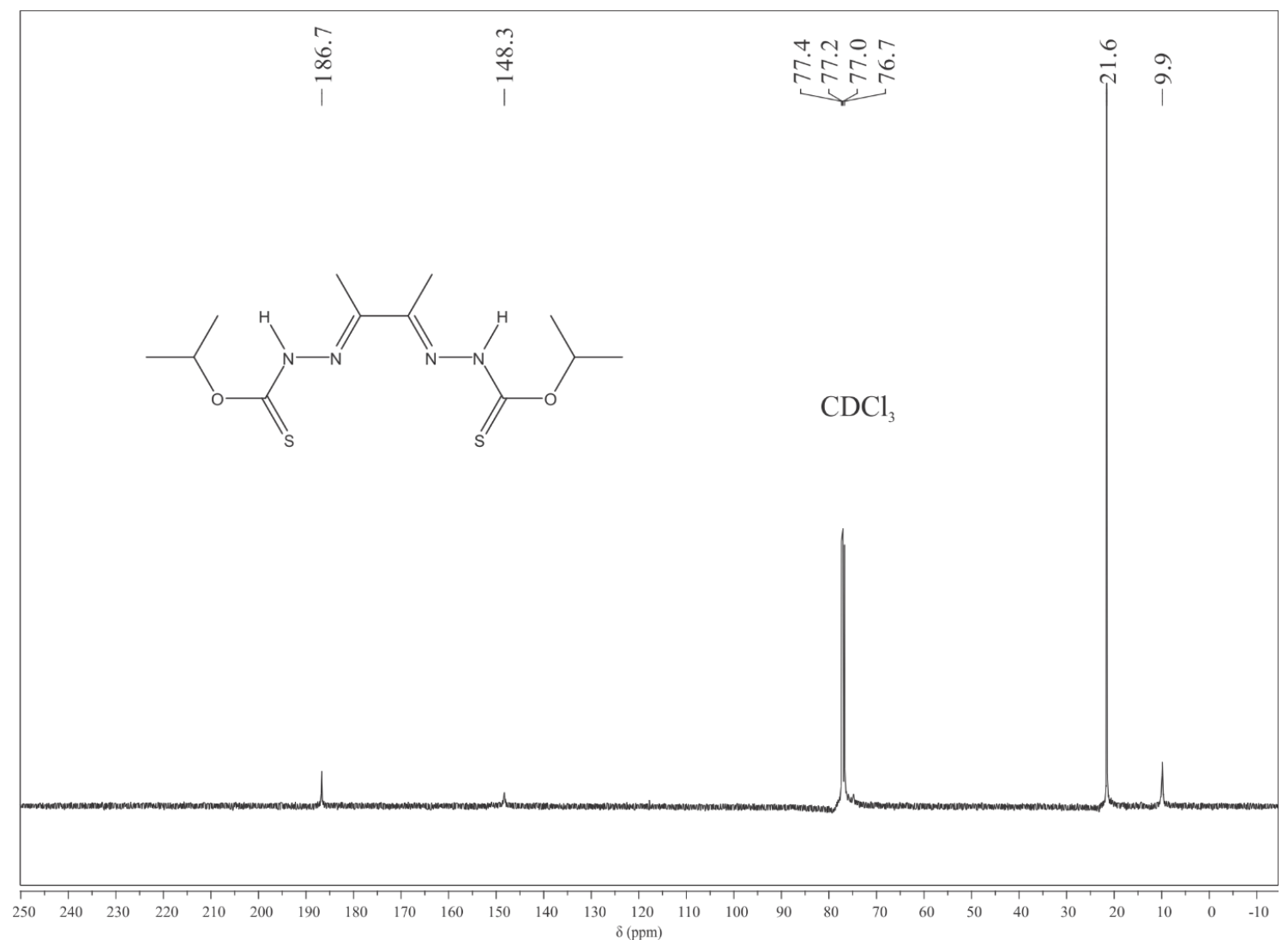

Figure A5. $\mathrm{H}_{2}{ }^{i} \mathrm{PTCB}(7){ }^{13} \mathrm{C} \mathrm{NMR}$

$\left(\mathrm{CDCl}_{3}, 100 \mathrm{MHz}\right) \delta 9.9,21.6,148.3,186.7$. 


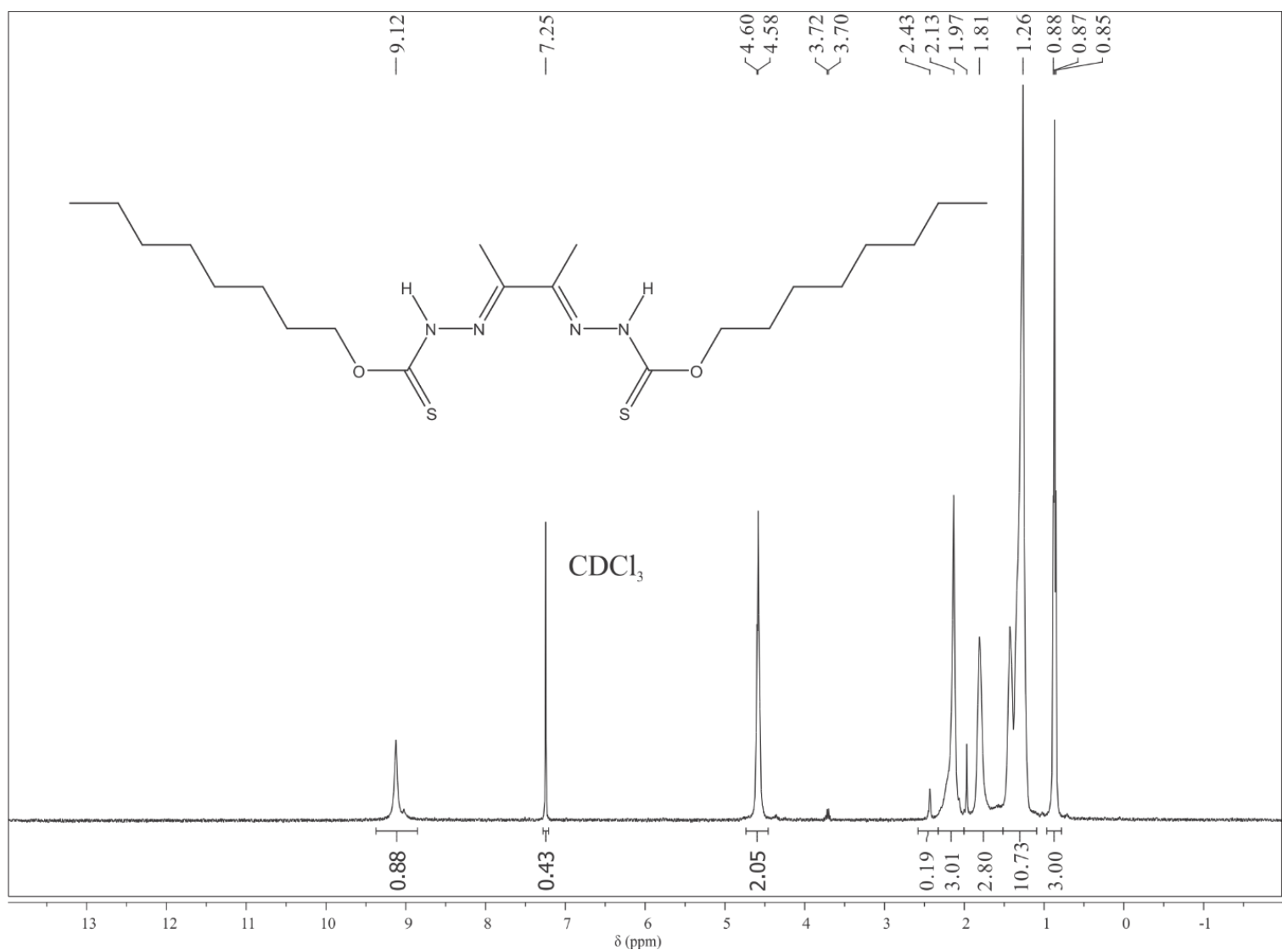

Figure A6. $\mathrm{H}_{2} \mathrm{OTCB}(8){ }^{1} \mathrm{H}$ NMR

$\left(\mathrm{CDCl}_{3}, 400 \mathrm{MHz}\right) \delta 0.87(\mathrm{br}, 6 \mathrm{H}) 1.28(\mathrm{~m}, 16 \mathrm{H}) 1.43(\mathrm{br}, 4 \mathrm{H}) 1.81(\mathrm{br}, 4 \mathrm{H}) 2.13(\mathrm{~s}, 6 \mathrm{H})$ 4.59 (br, 4H) 9.12 (s, 2H). 


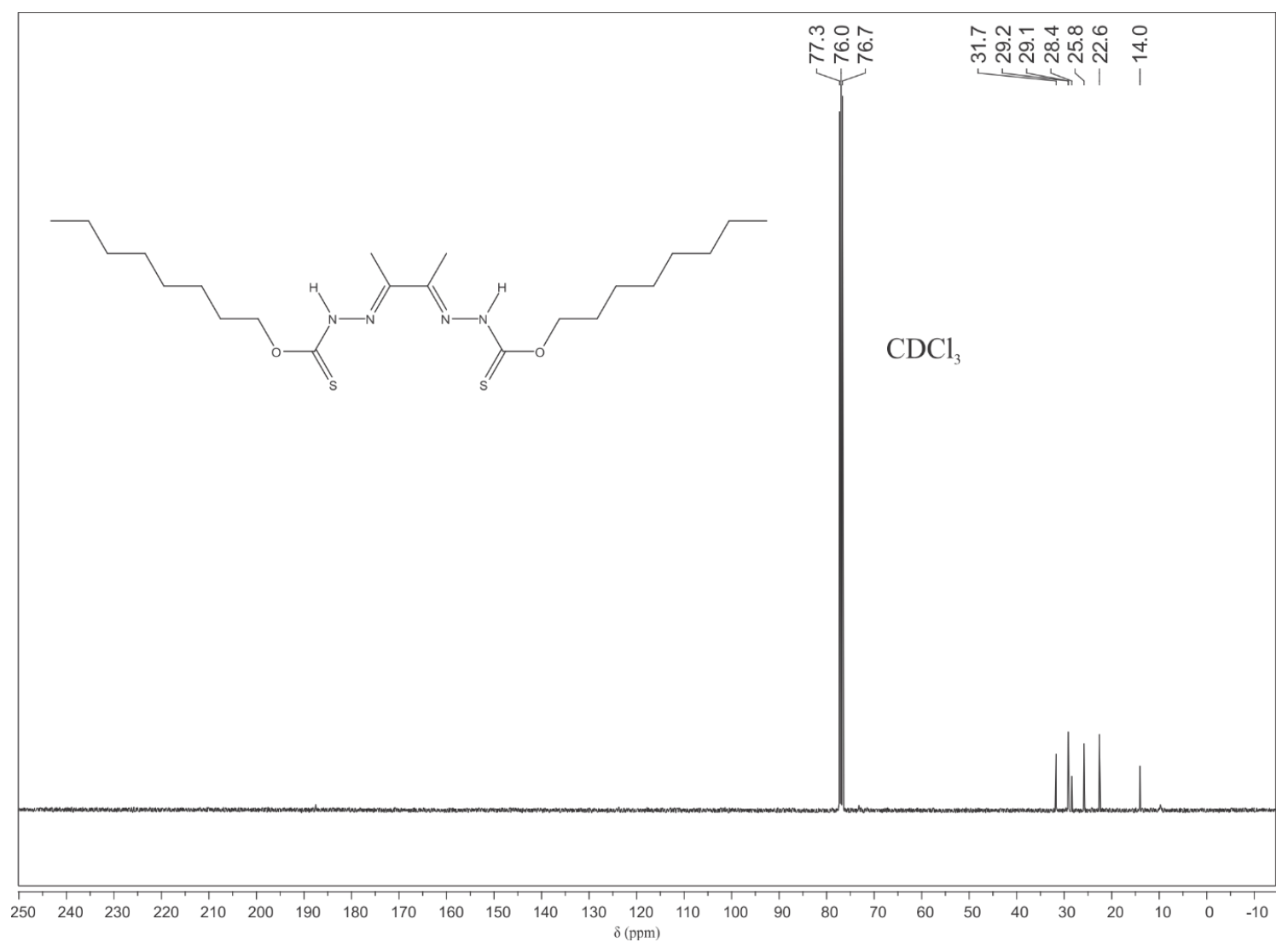

Figure A7. $\mathrm{H}_{2} \mathrm{OTCB}(8){ }^{13} \mathrm{C}$ NMR

$\left(\mathrm{CDCl}_{3}, 100 \mathrm{MHz}\right) \delta 14.1,22.6,25.8,28.4,29.1,31.7$. 


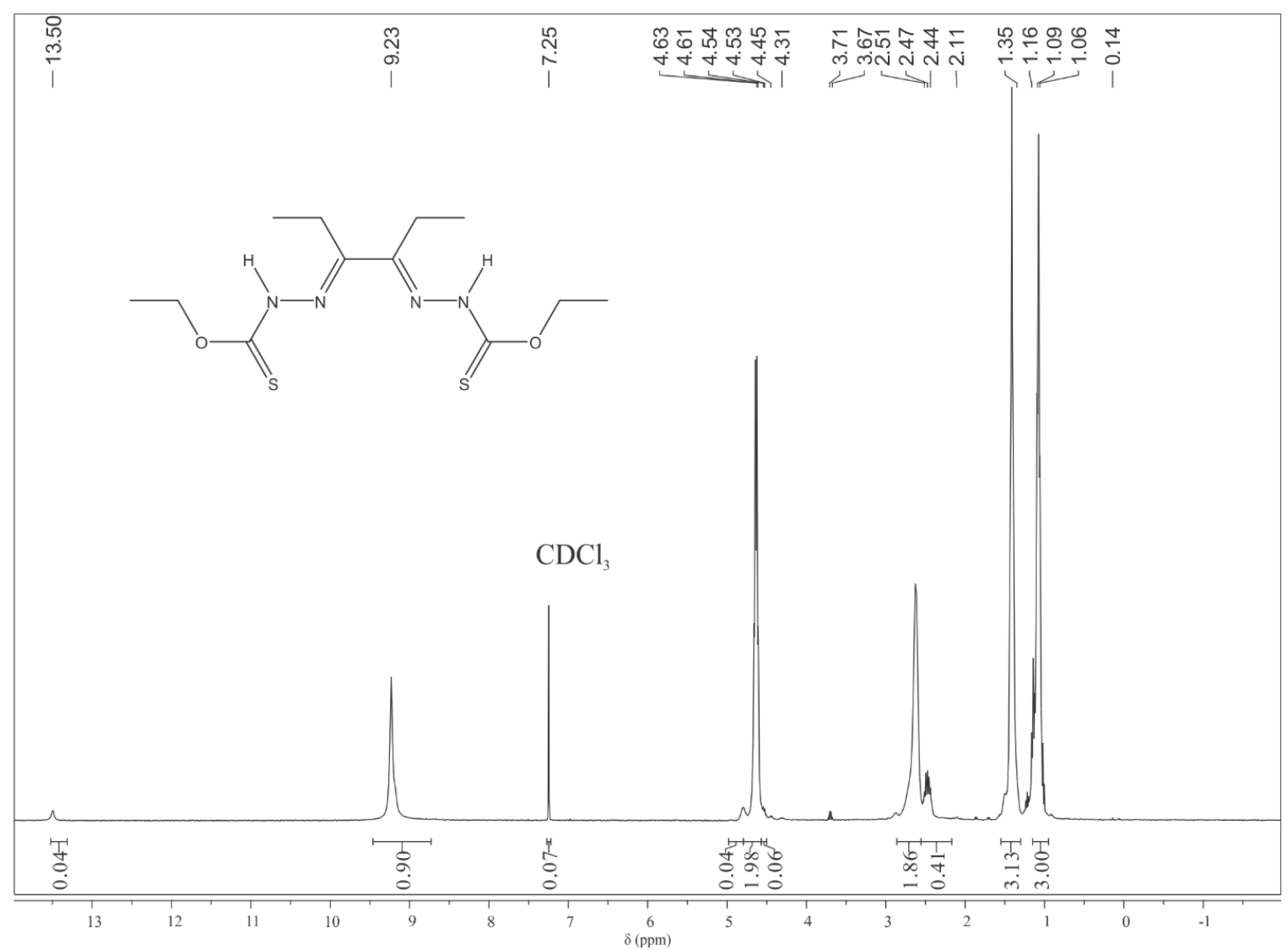

Figure A8. $\mathrm{H}_{2} \mathrm{ETCH}(9){ }^{1} \mathrm{H}$ NMR

$\left(\mathrm{CDCl}_{3}, 400 \mathrm{MHz}\right) \delta 1.08$ (br, 6H) 1.41 (br, 6H) 2.63 (br, 4H) 4.63 (br, 4H) 9.23 (br, 2H). 


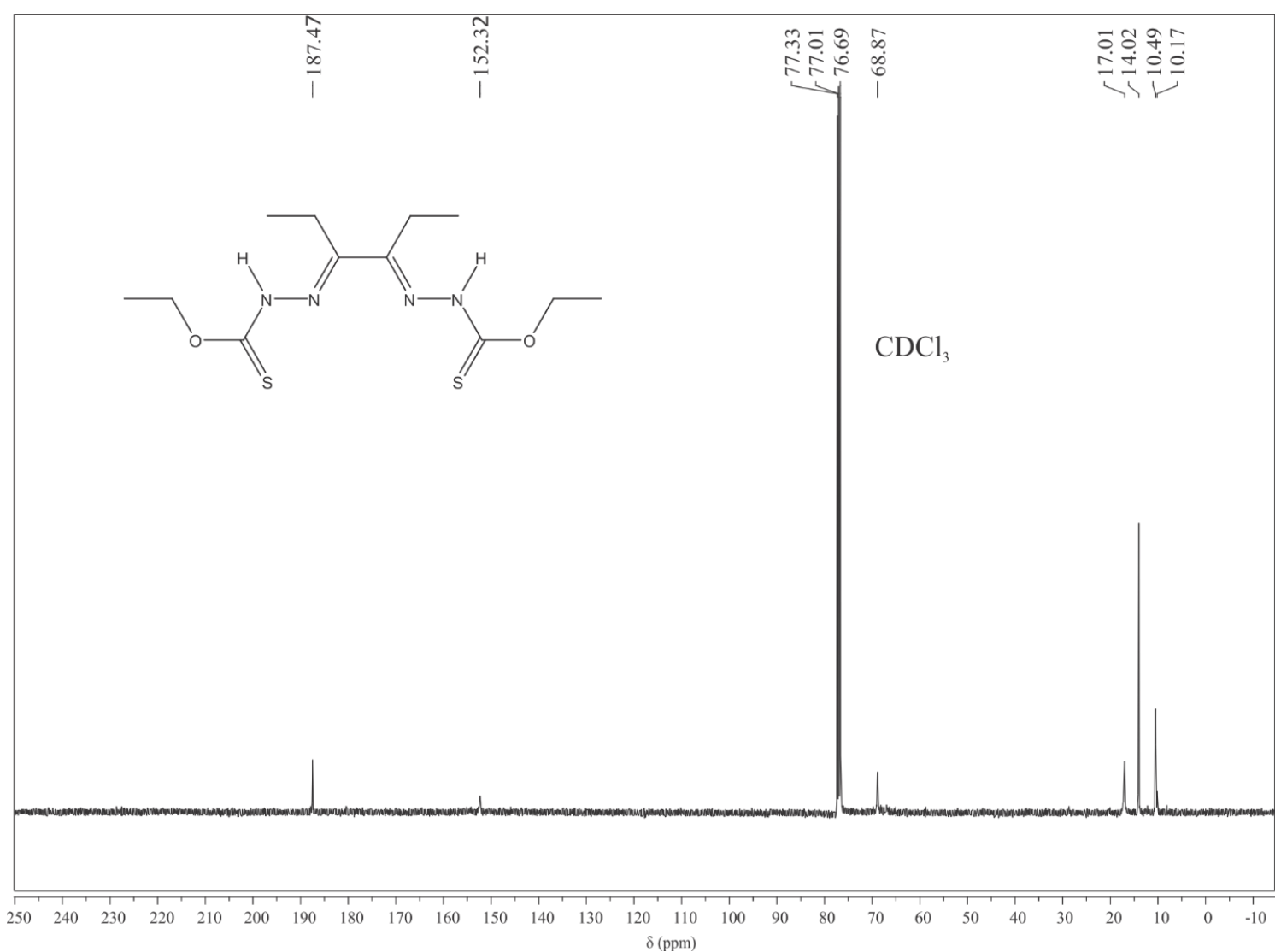

Figure A9. $\mathrm{H}_{2} \mathrm{ETCH}(9){ }^{13} \mathrm{C}$ NMR

$\left(\mathrm{CDCl}_{3}, 100 \mathrm{MHz}\right) \delta 10.49,14.02,17.01,68.87,152.27,187.47$. 


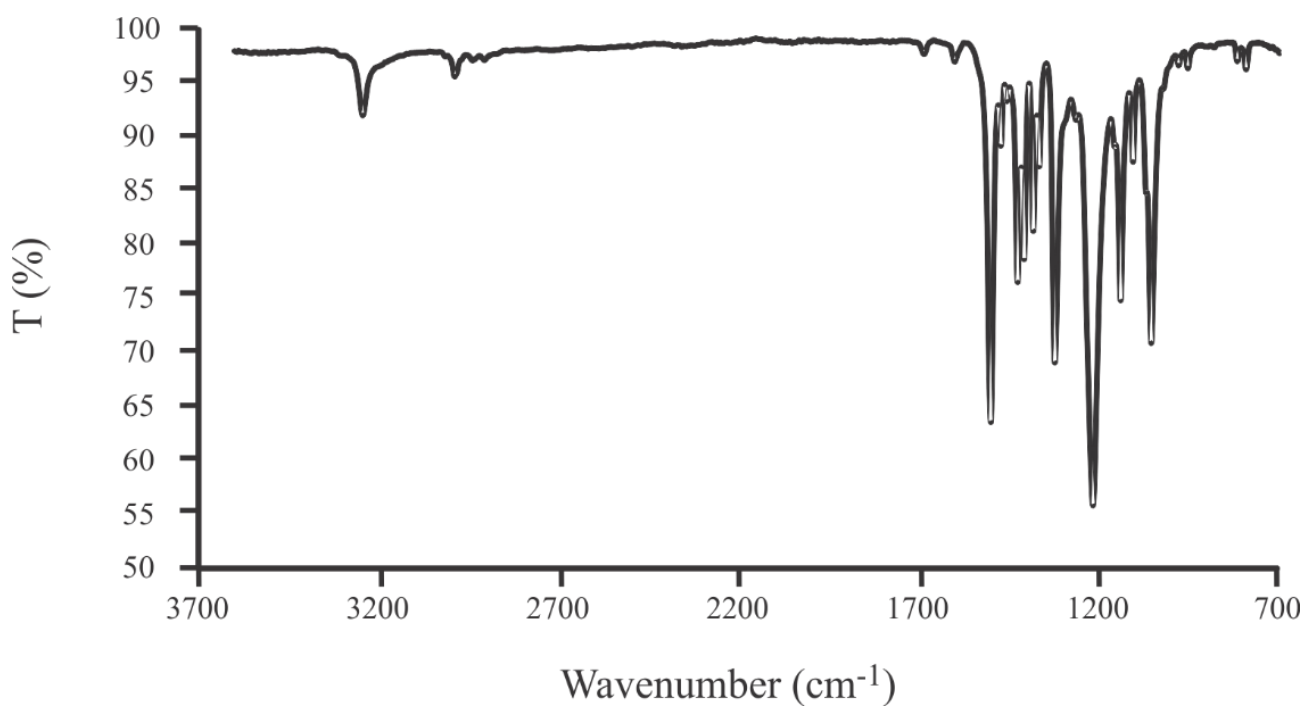

Figure A10: FT-IR of $\mathrm{H}_{2} \mathrm{ETCB}$ (5)

Infrared spectrum of the $\mathrm{H}_{2} \mathrm{ETCB}$ ligand as a powder (ATR)

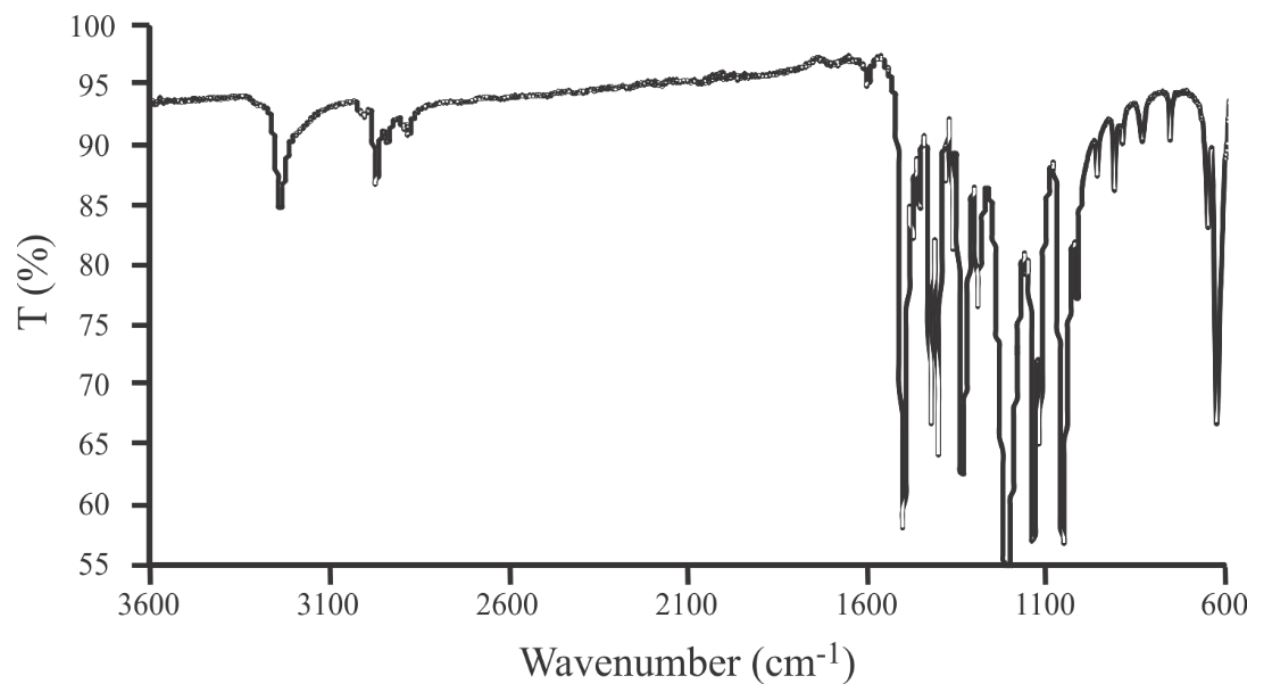

Figure A11: FT-IR of $\mathrm{H}_{2} \mathrm{PTCB}$ (6)

Infrared spectrum of the $\mathrm{H}_{2} \mathrm{PTCB}$ ligand as a powder (ATR) 


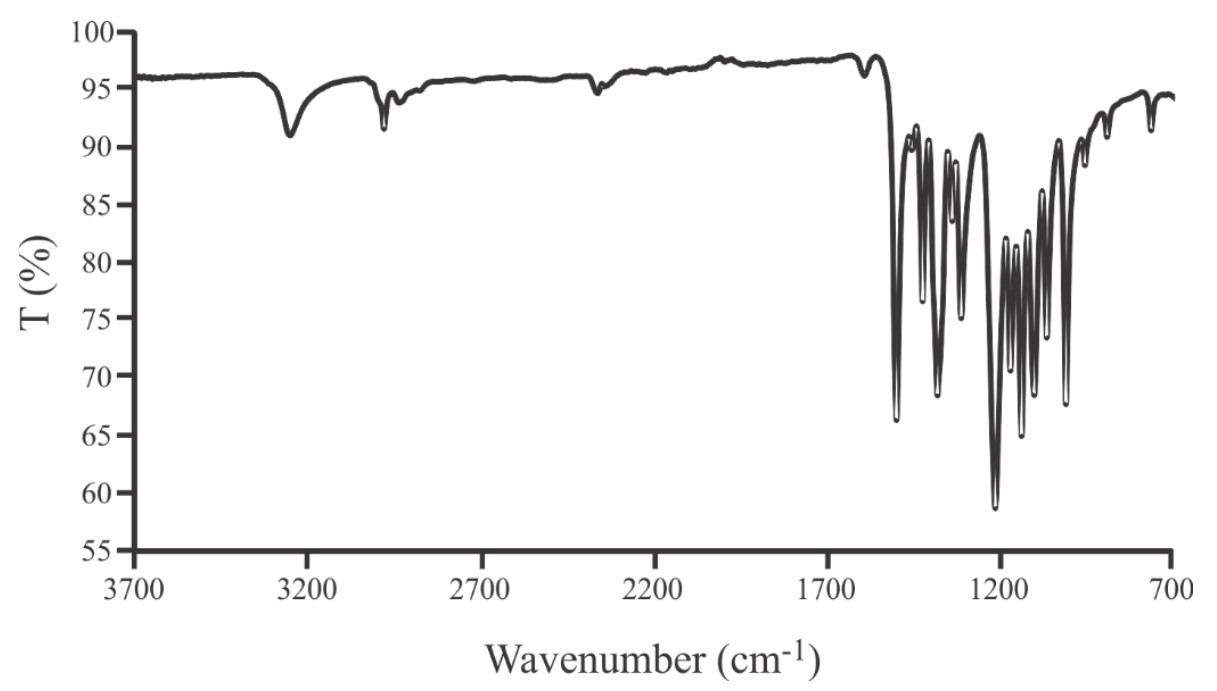

Figure A12: FT-IR of $\mathrm{H}_{2}{ }^{i} \mathrm{PTCB}$ (7)

Infrared spectrum of the $\mathrm{H}_{2}{ }^{i} \mathrm{PTCB}$ ligand as a powder (ATR)

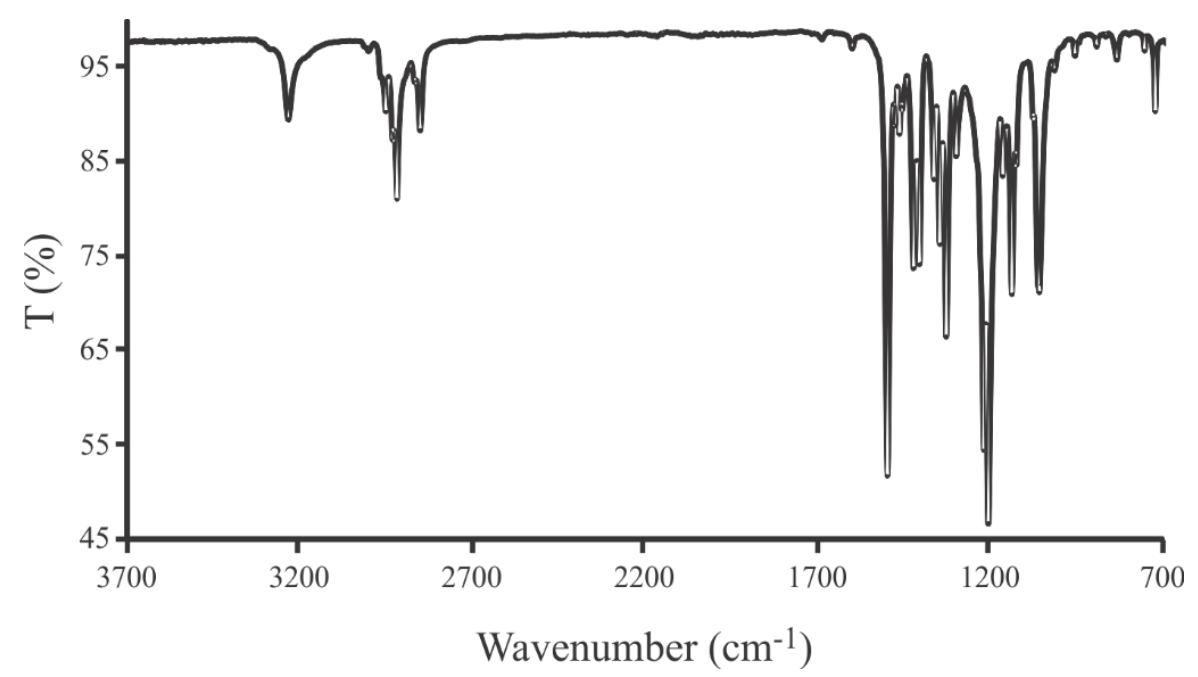

Figure A13: FT-IR of $\mathrm{H}_{2} \mathrm{OTCB}$ (8)

Infrared spectrum of the $\mathrm{H}_{2} \mathrm{OTCB}$ ligand as a powder (ATR) 


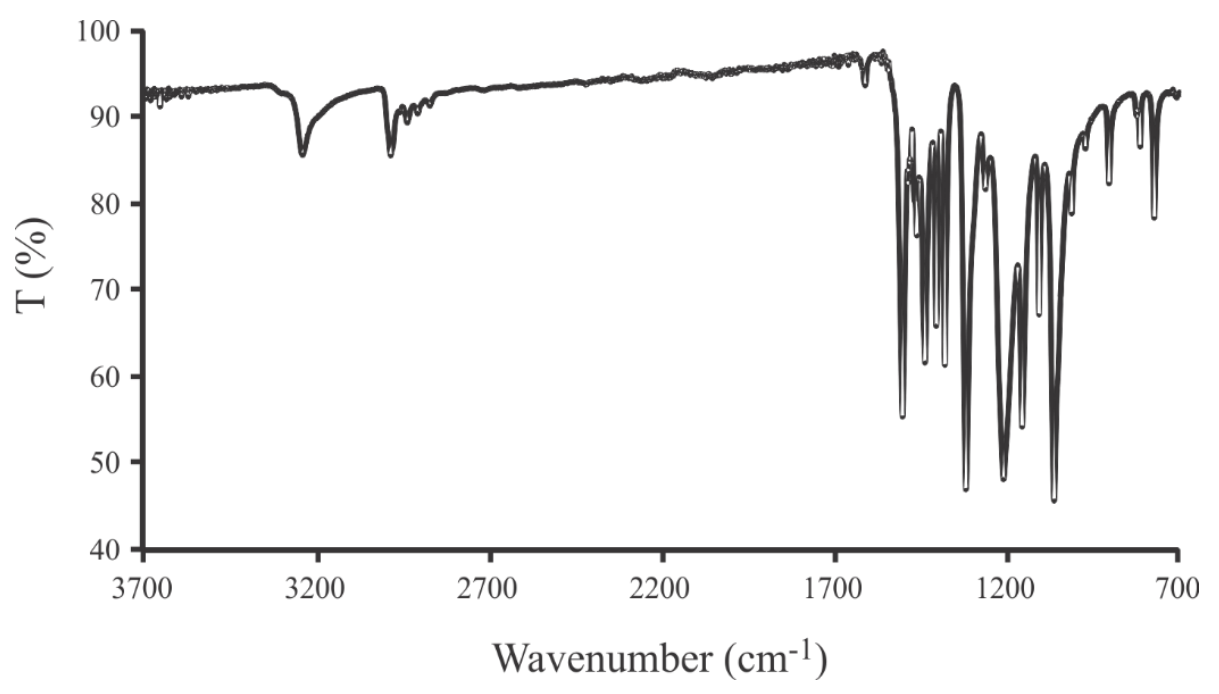

Figure A14: FT-IR of $\mathrm{H}_{2} \mathrm{ETCH}$ (9)

Infrared spectrum of the $\mathrm{H}_{2} \mathrm{ETCH}$ ligand as a powder (ATR)

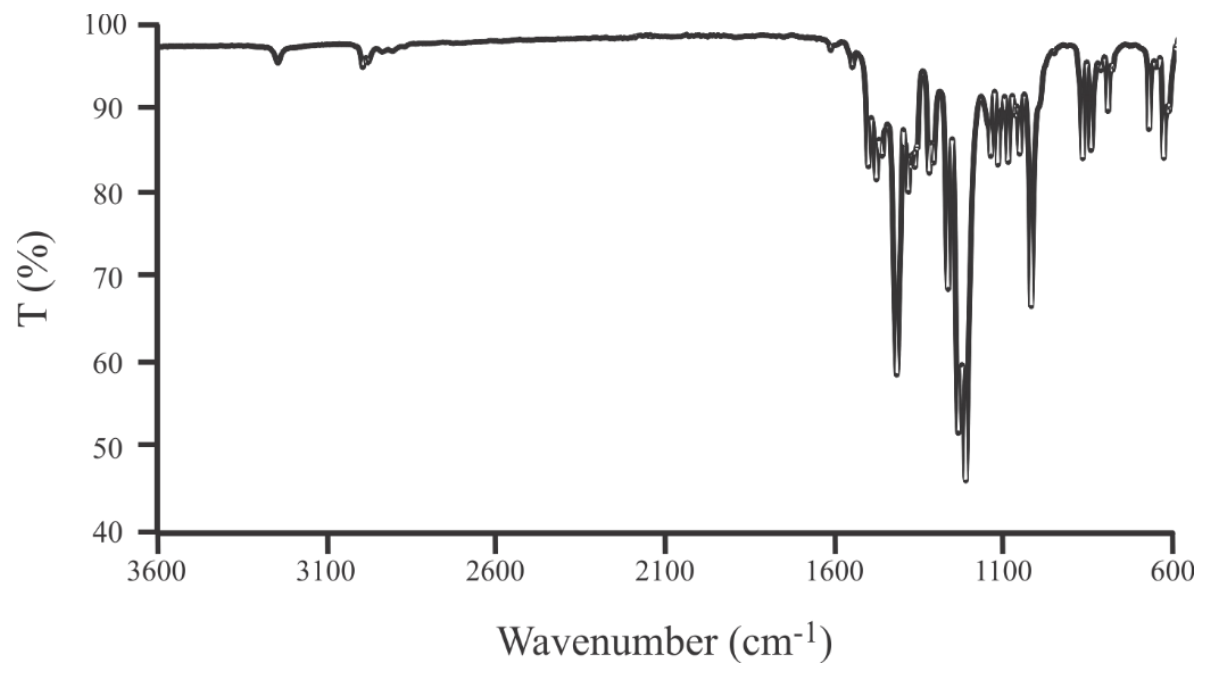

Figure A15: FT-IR of $\mathrm{Cu}(\mathrm{ETCB})$ (11)

Infrared spectrum of the $\mathrm{Cu}(\mathrm{ETCB})$ ligand as a powder (ATR) 


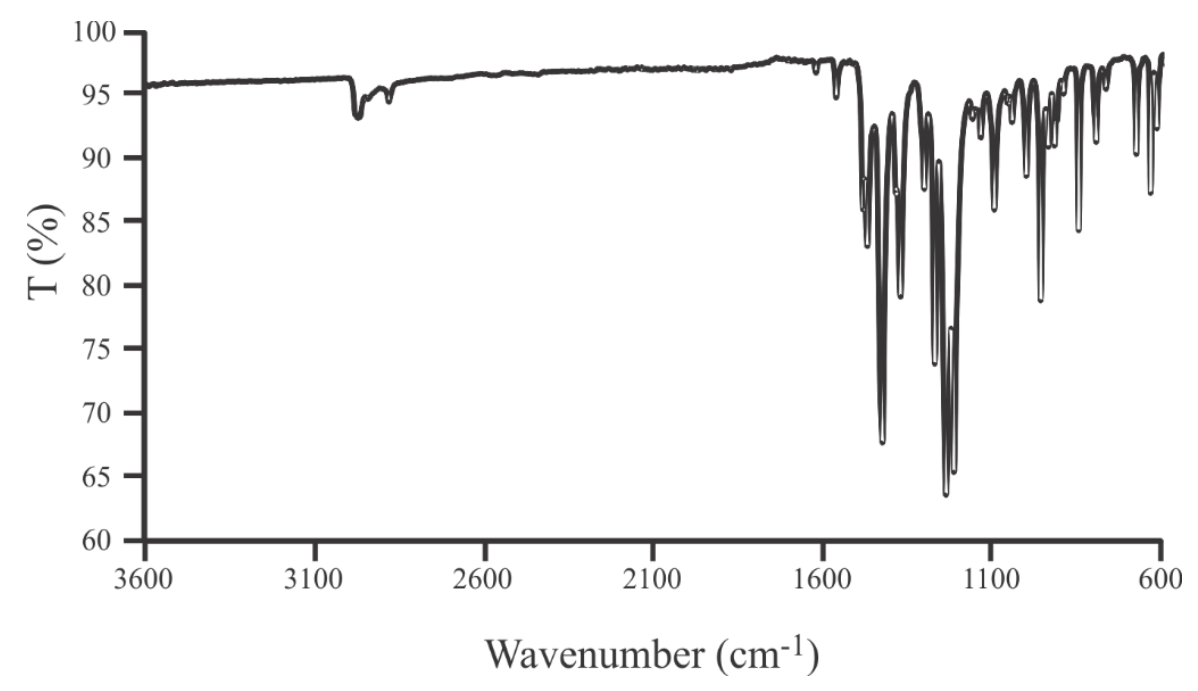

Figure A16: IR Spectrum of $\mathrm{Cu}(\mathrm{PTCB})(\mathbf{1 2})$

Infrared spectrum of $\mathrm{Cu}(\mathrm{PTCB})$ as a powder (ATR)

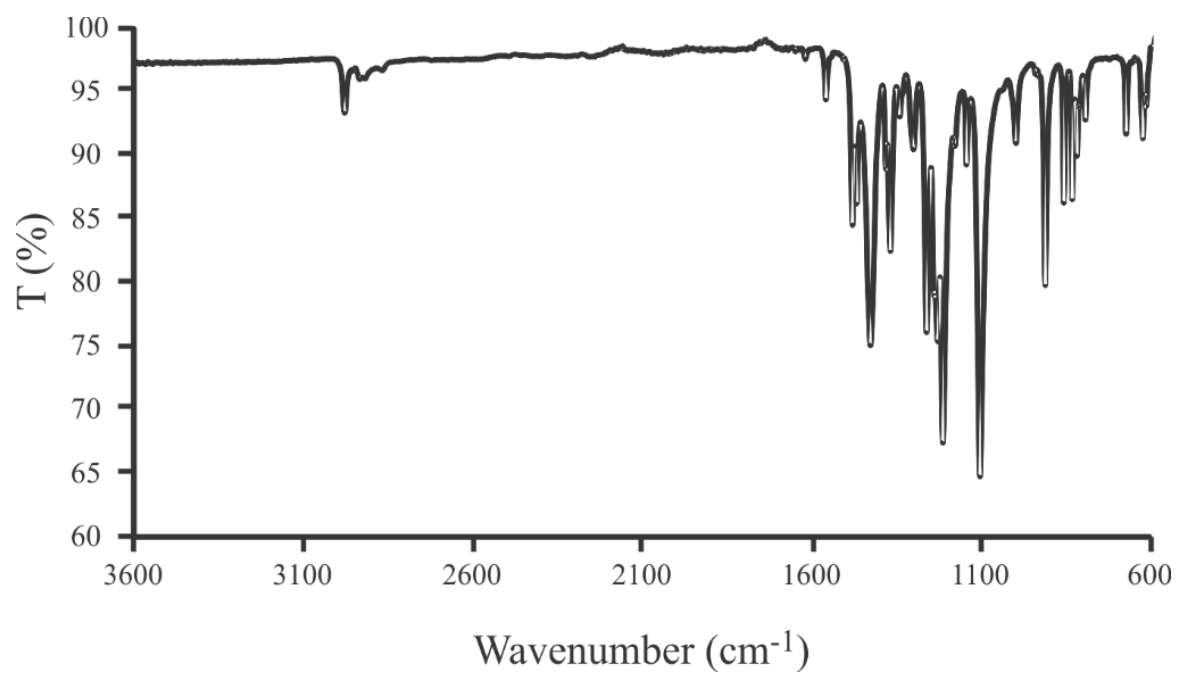

Figure A17: IR Spectrum of $\mathrm{Cu}\left({ }^{i} \mathrm{PTCB}\right)(\mathbf{1 3})$

Infrared spectrum of $\mathrm{Cu}\left({ }^{i} \mathrm{PTCB}\right)$ as a powder (ATR) 


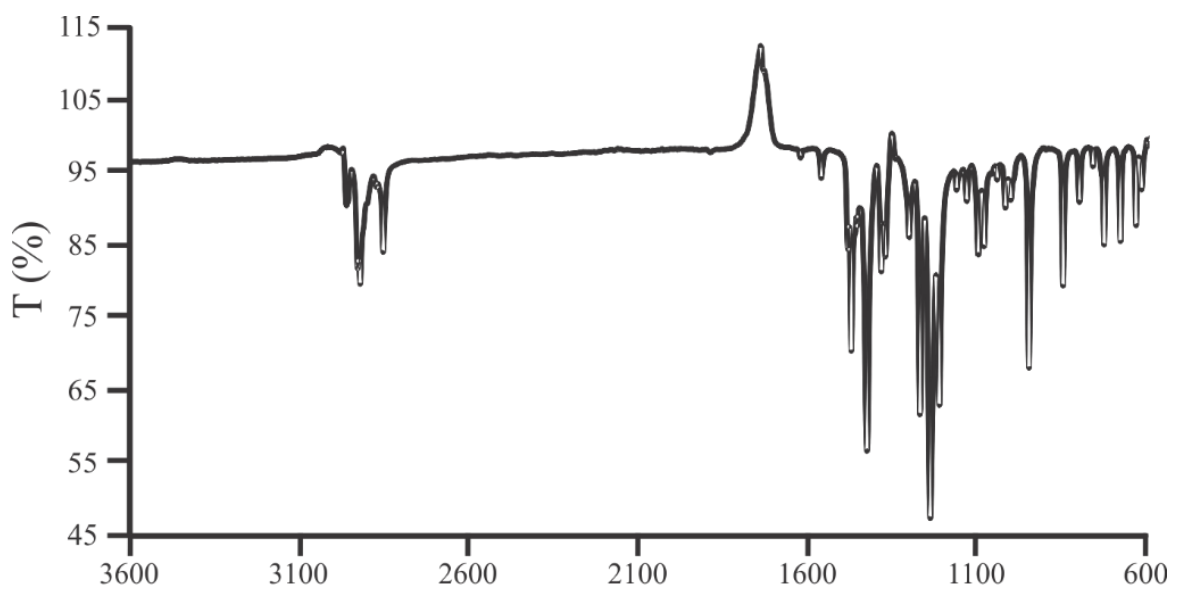

Wavenumber $\left(\mathrm{cm}^{-1}\right)$

Figure A18: IR Spectrum of $\mathrm{Cu}(\mathrm{OTCB})(\mathbf{1 4})$

Infrared spectrum of $\mathrm{Cu}(\mathrm{OTCB})$ as a powder (ATR)

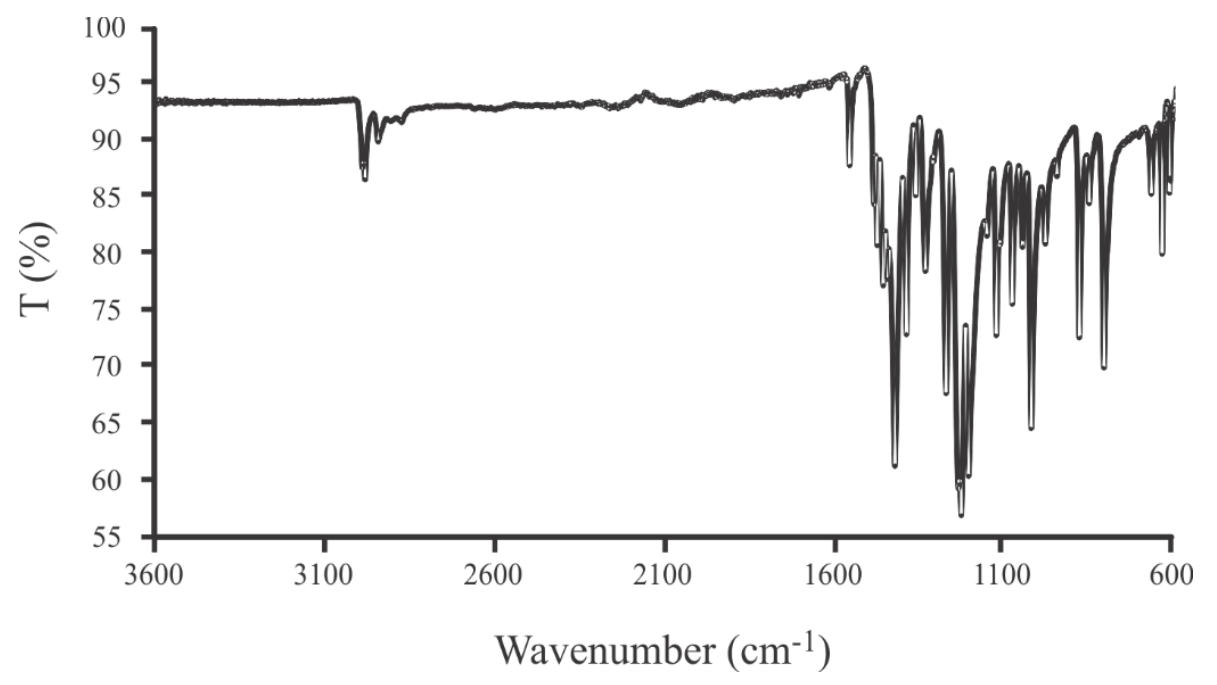

Figure A19: IR Spectrum of $\mathrm{Cu}(\mathrm{ETCH})(\mathbf{1 5})$

Infrared spectrum of $\mathrm{Cu}(\mathrm{ETCH})$ as a powder (ATR) 


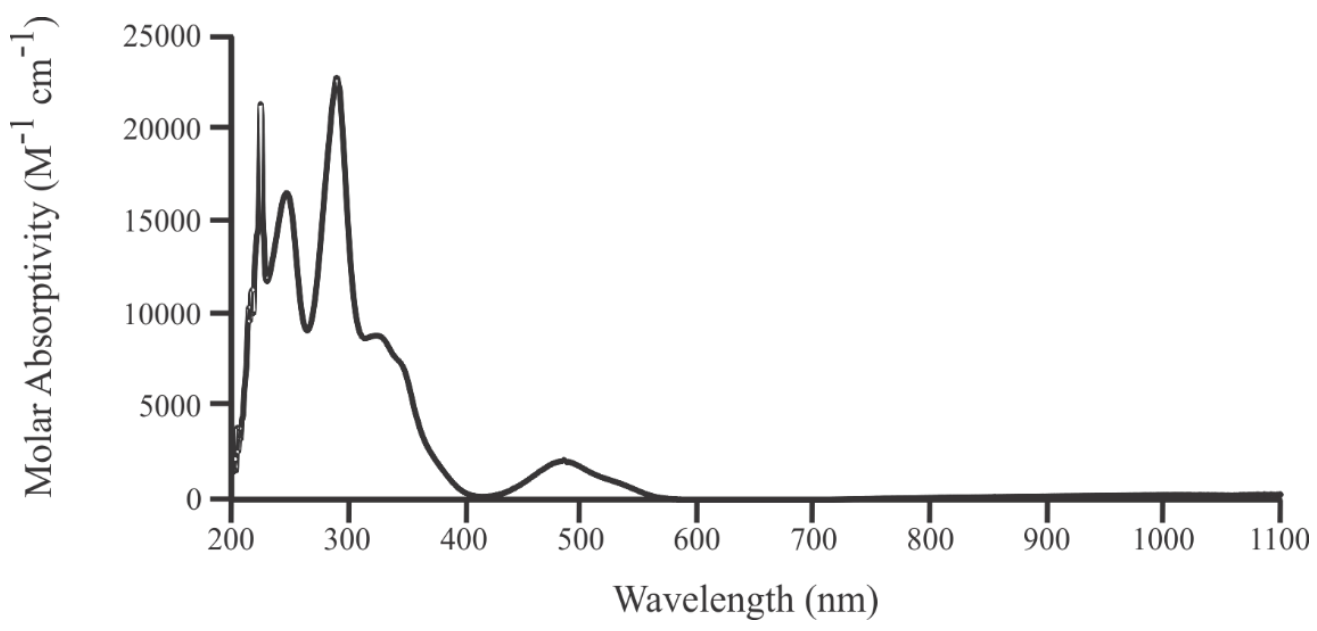

Figure A20. $\mathrm{Cu}$ (ETCB) (11) UV-Vis in dichloromethane

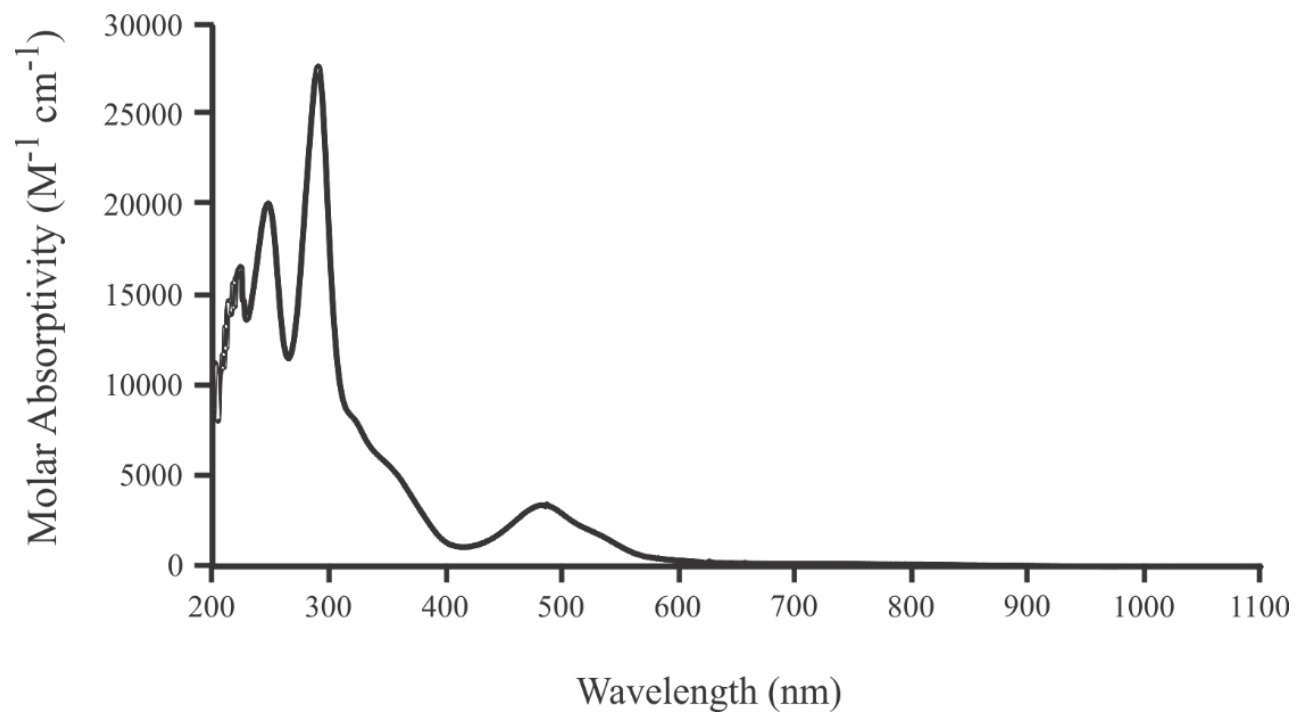

Figure A21. Cu(PTCB) (12) UV-Vis in dichloromethane 


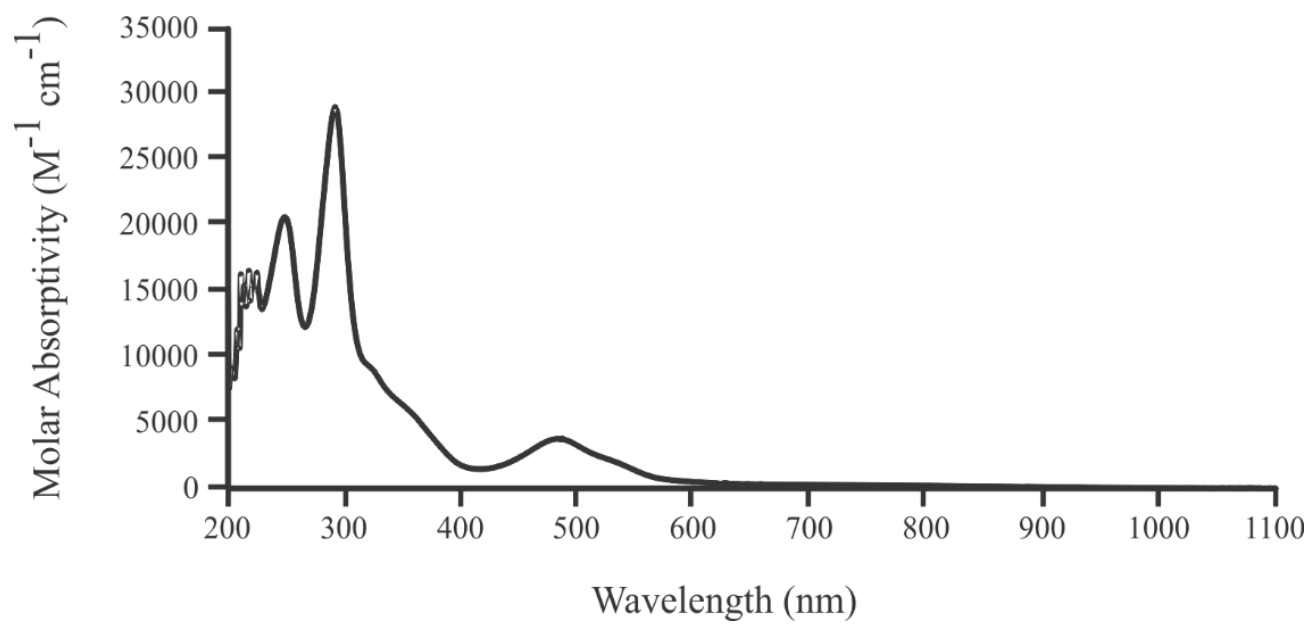

Figure A22. $\mathrm{Cu}\left({ }^{i} \mathrm{PTCB}\right)(\mathbf{1 3}) \mathrm{UV}-\mathrm{V}$ is in dichloromethane

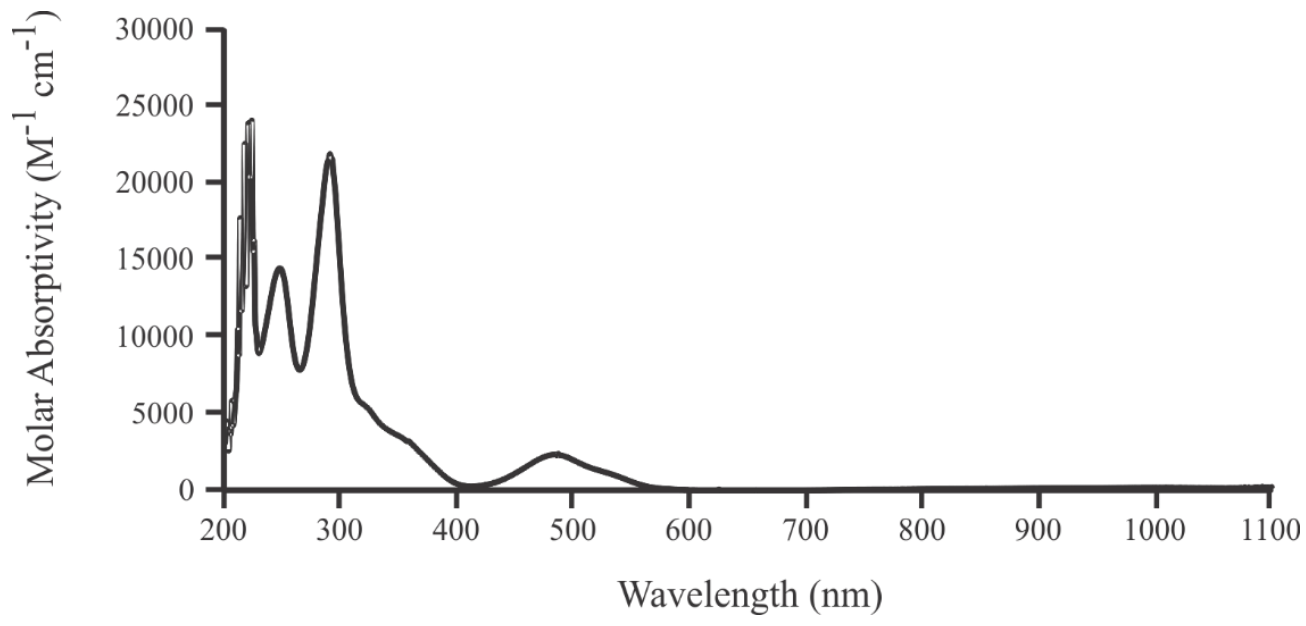

Figure A23. $\mathrm{Cu}(\mathrm{OTCB})$ (14) UV-Vis in dichloromethane 


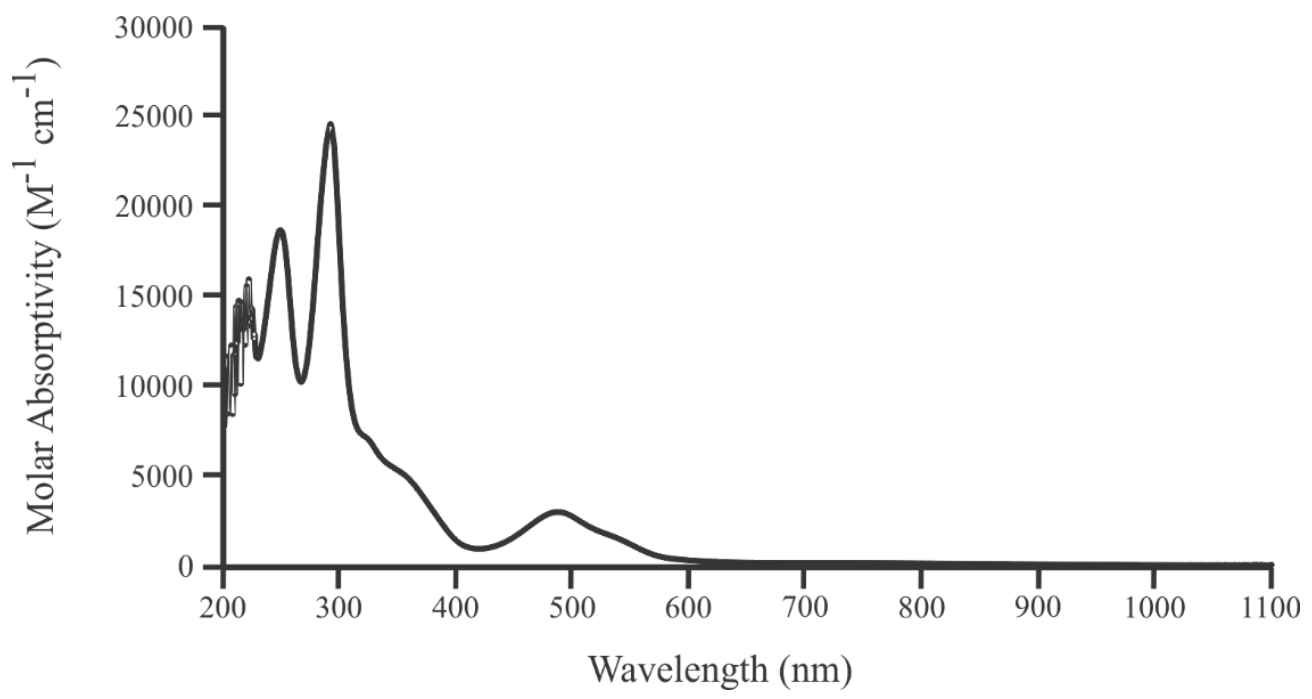

Figure A24. $\mathrm{Cu}(\mathrm{ETCH})(\mathbf{1 5}) \mathrm{UV}$-Vis in dichloromethane

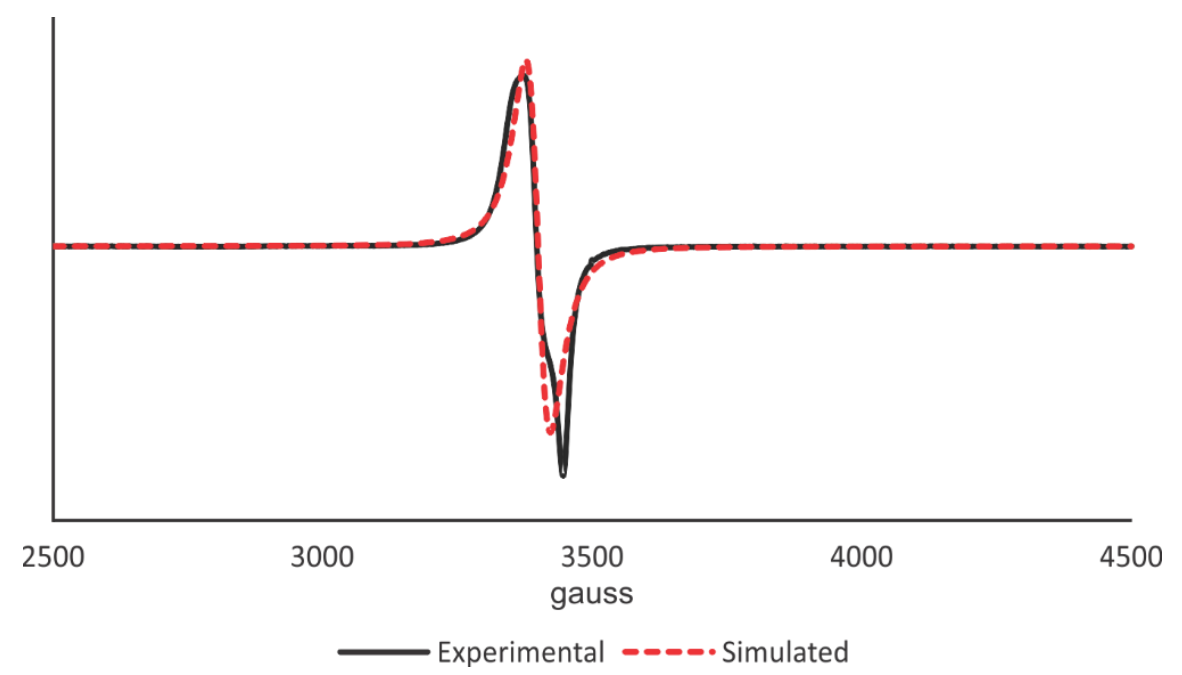

Figure A25. Cu(MTCB) (10) EPR on powder at room temperature 


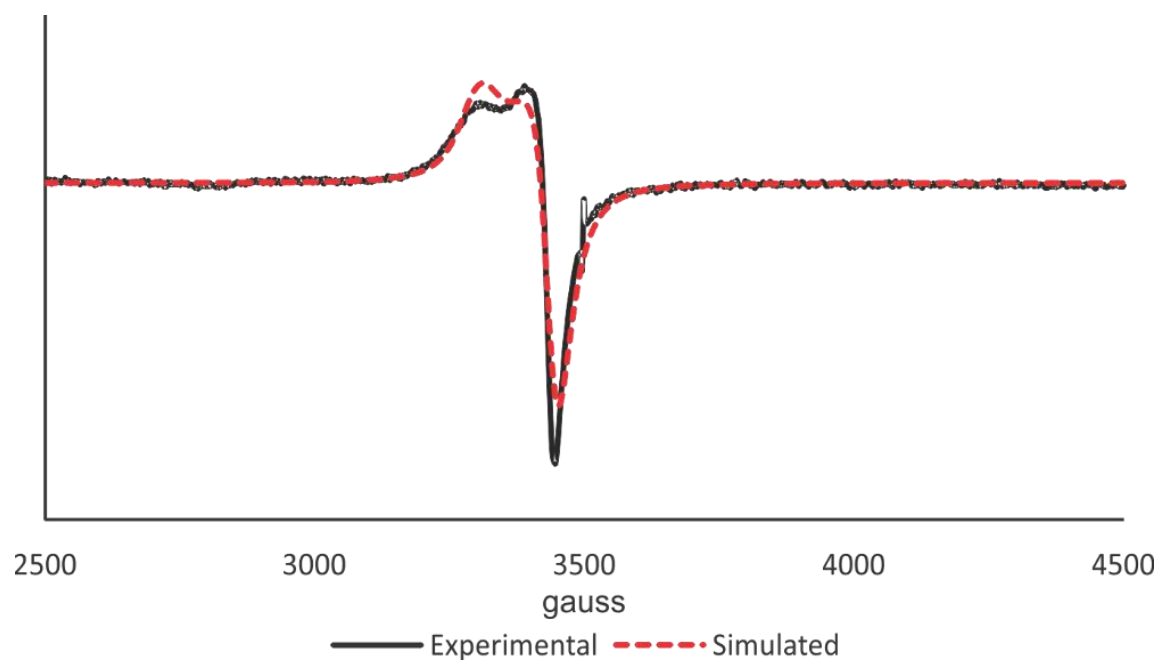

Figure A26. $\mathrm{Cu}(\mathrm{ETCB})$ (11) EPR on powder at room temperature

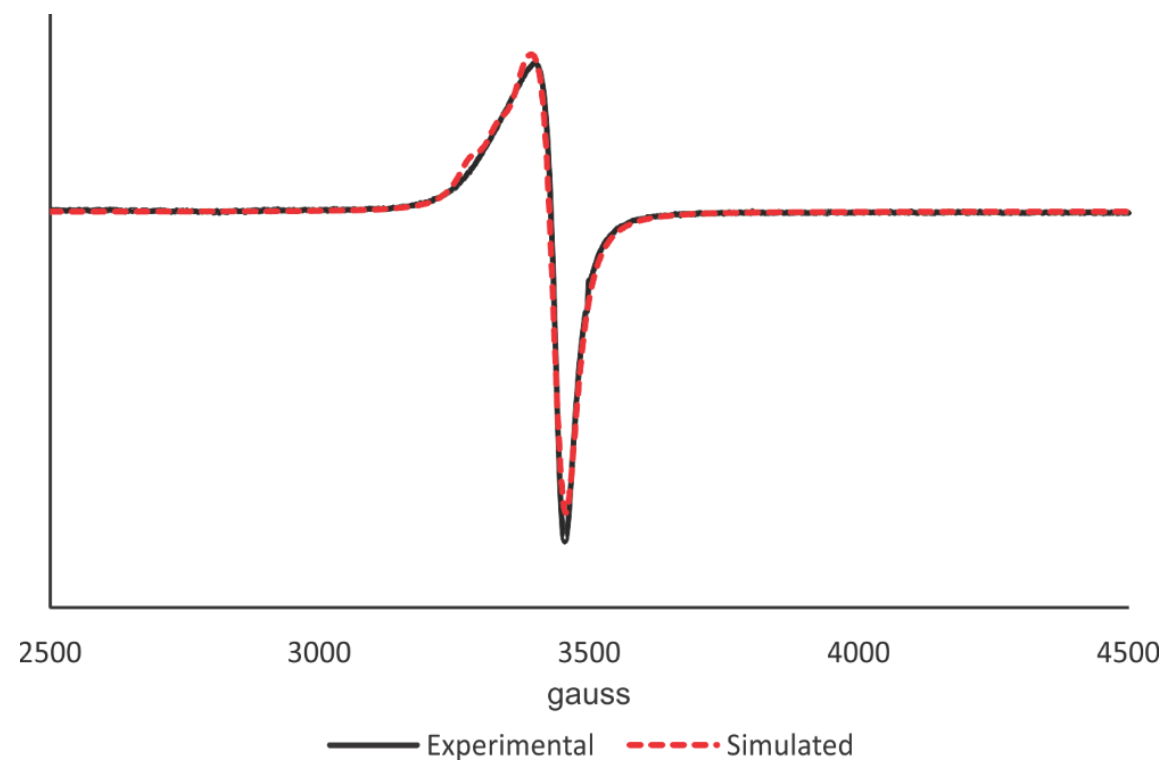

Figure A27. $\mathrm{Cu}\left({ }^{i} \mathrm{PTCB}\right)(\mathbf{1 3}) \mathrm{EPR}$ on powder at room temperature 


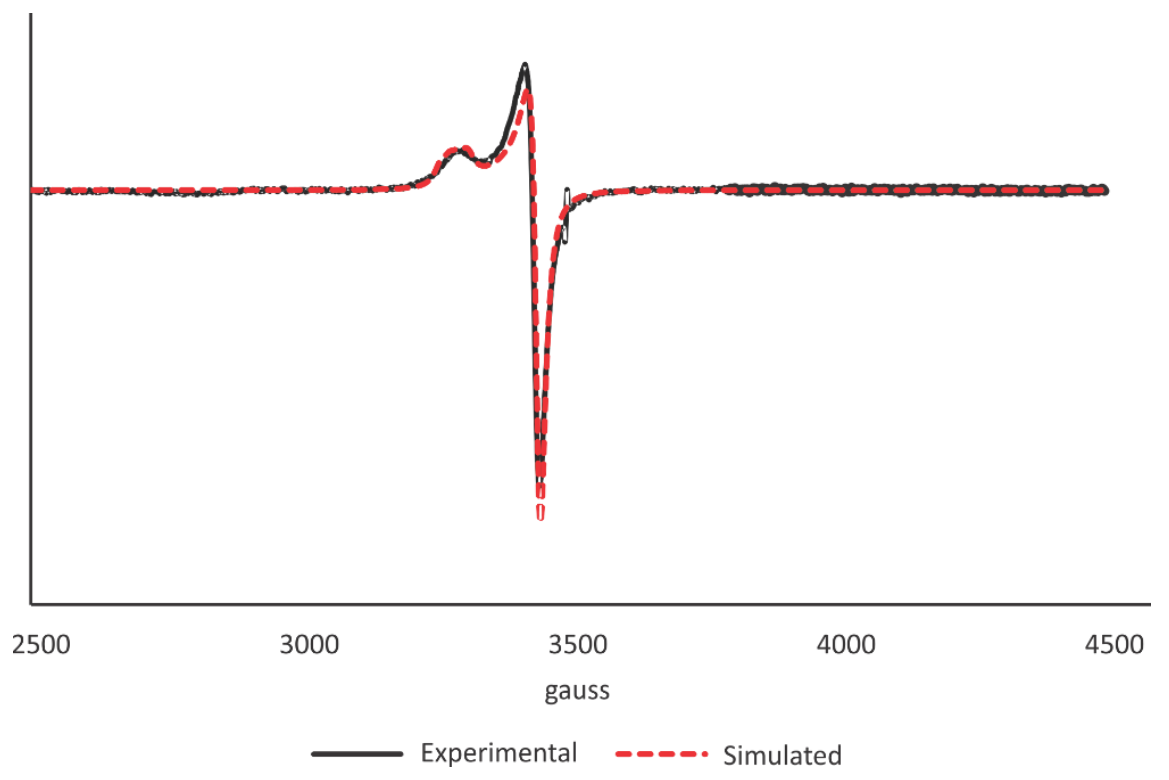

Figure A28. $\mathrm{Cu}(\mathrm{OTCB})$ (14) EPR on powder at room temperature

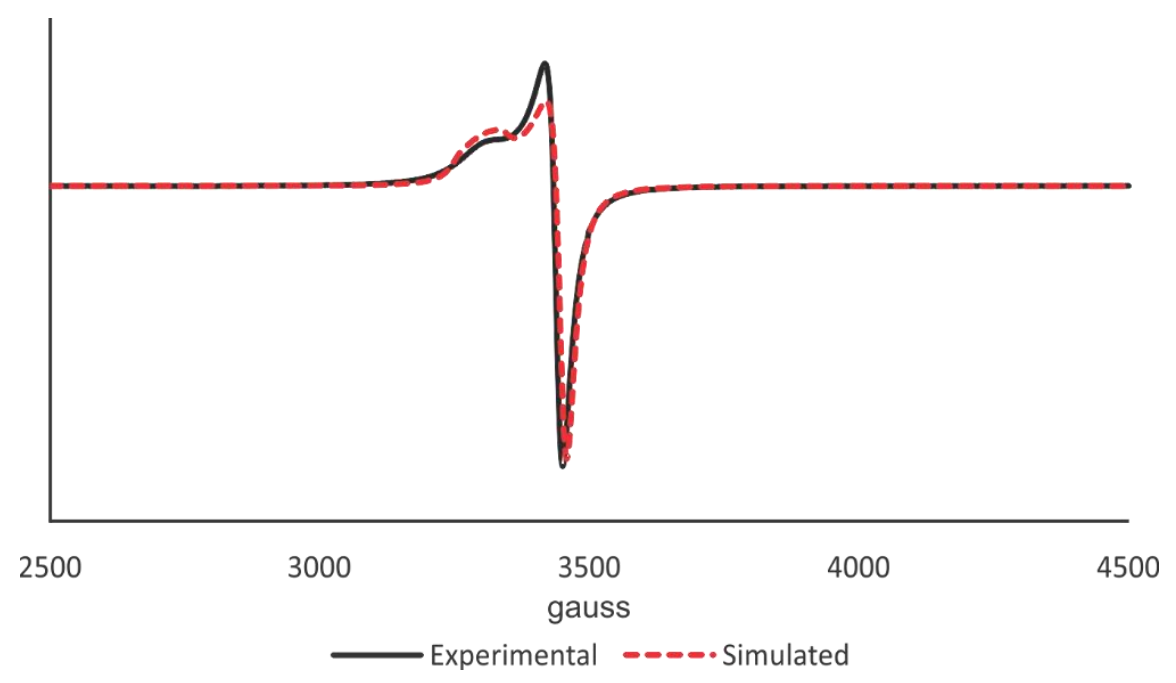

Figure A29. $\mathrm{Cu}(\mathrm{ETCH})(\mathbf{1 5}) \mathrm{EPR}$ on powder at room temperature 


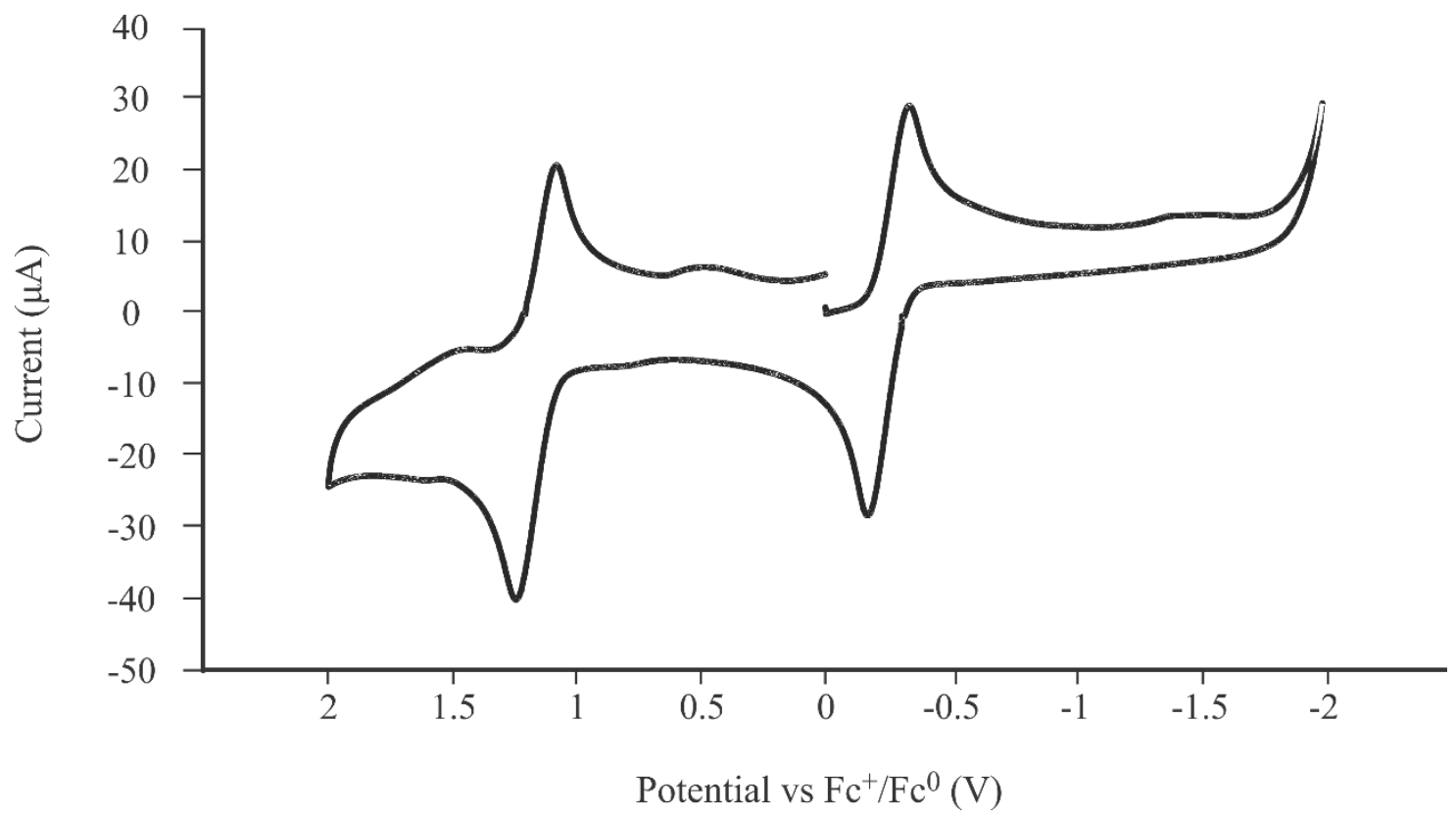

Figure A30. Cyclic voltammogram of $\mathbf{1 1}$ in DCM at $200 \mathrm{mV} / \mathrm{s}$

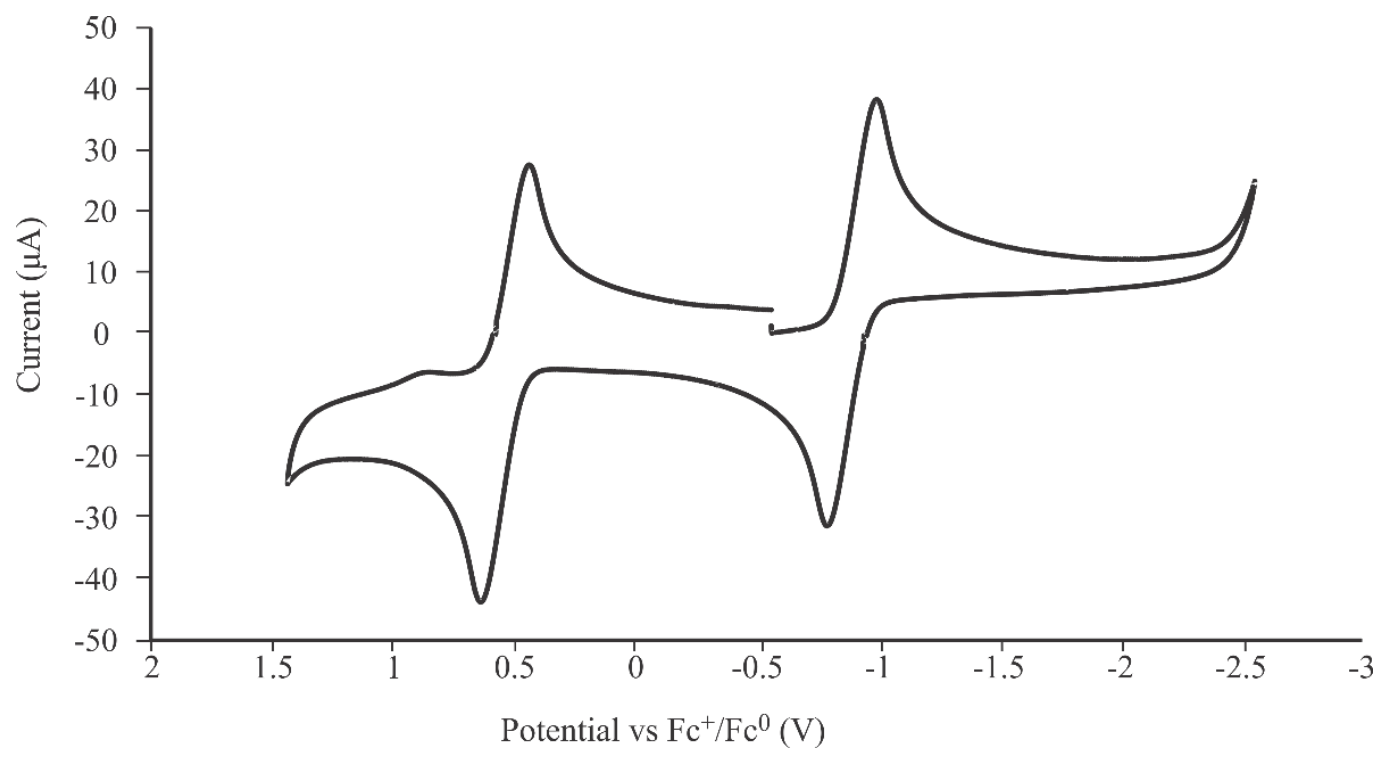

Figure A31. Cyclic voltammogram of $\mathbf{1 2}$ in DCM at $200 \mathrm{mV} / \mathrm{s}$ 


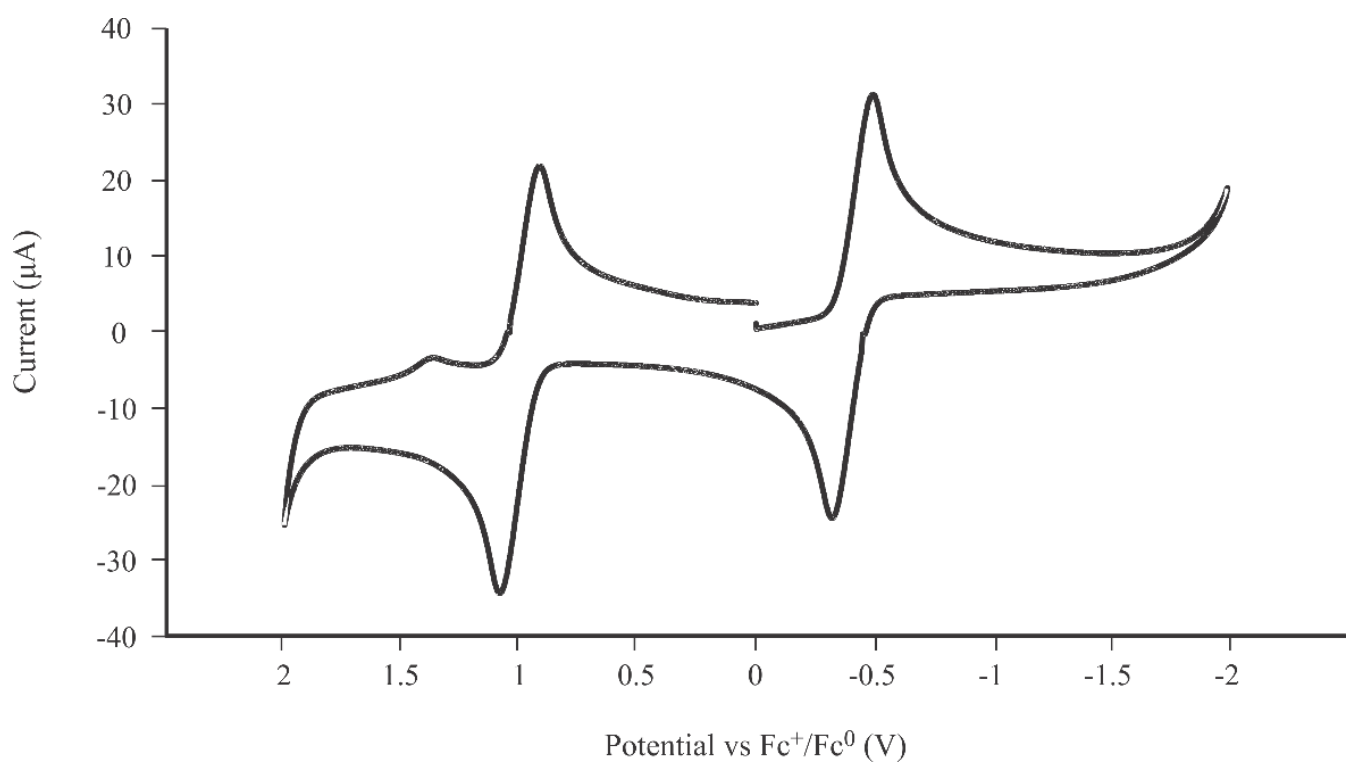

Figure A32. Cyclic voltammogram of $\mathbf{1 3}$ in DCM at $200 \mathrm{mV} / \mathrm{s}$

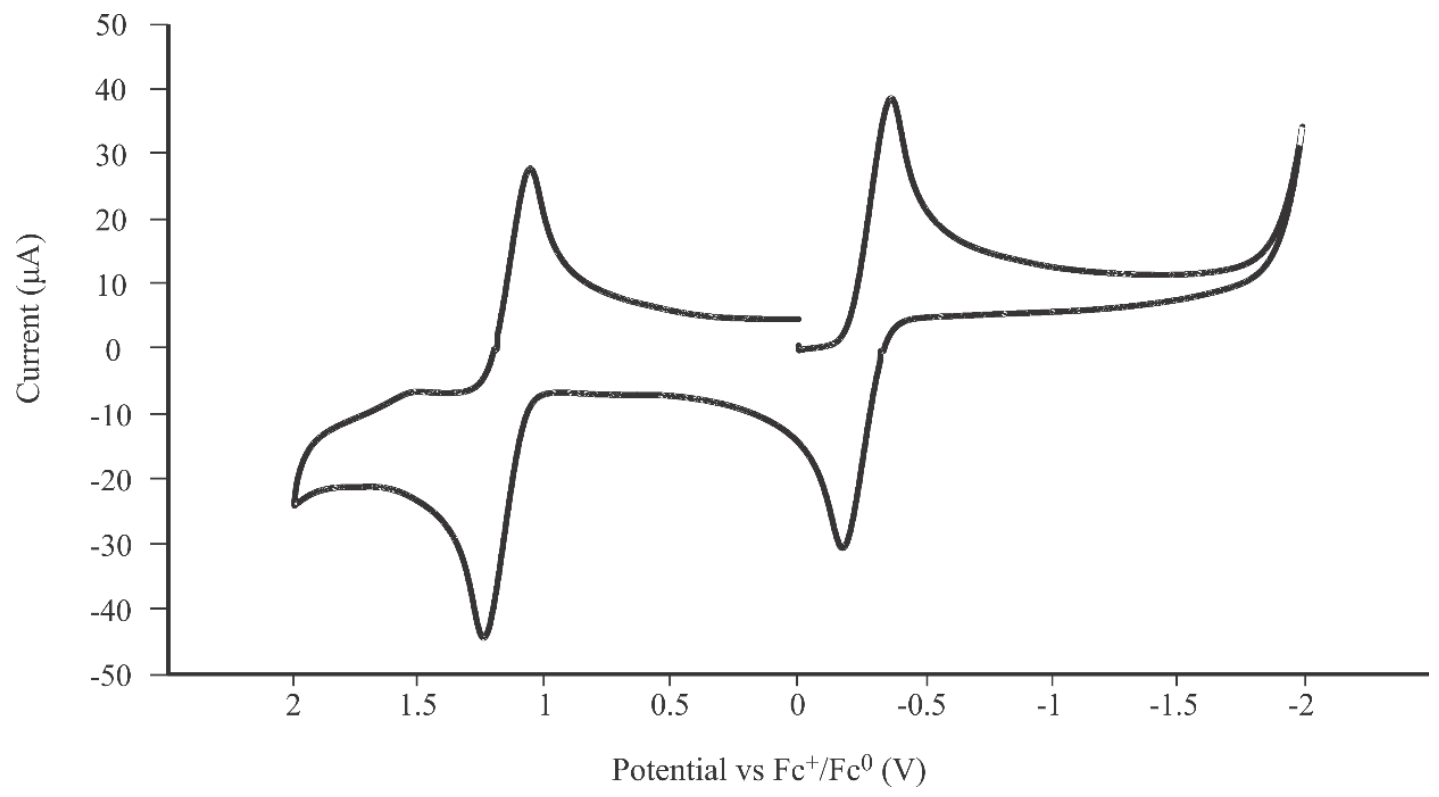

Figure A33. Cyclic voltammogram of 14 in DCM at $200 \mathrm{mV} / \mathrm{s}$ 


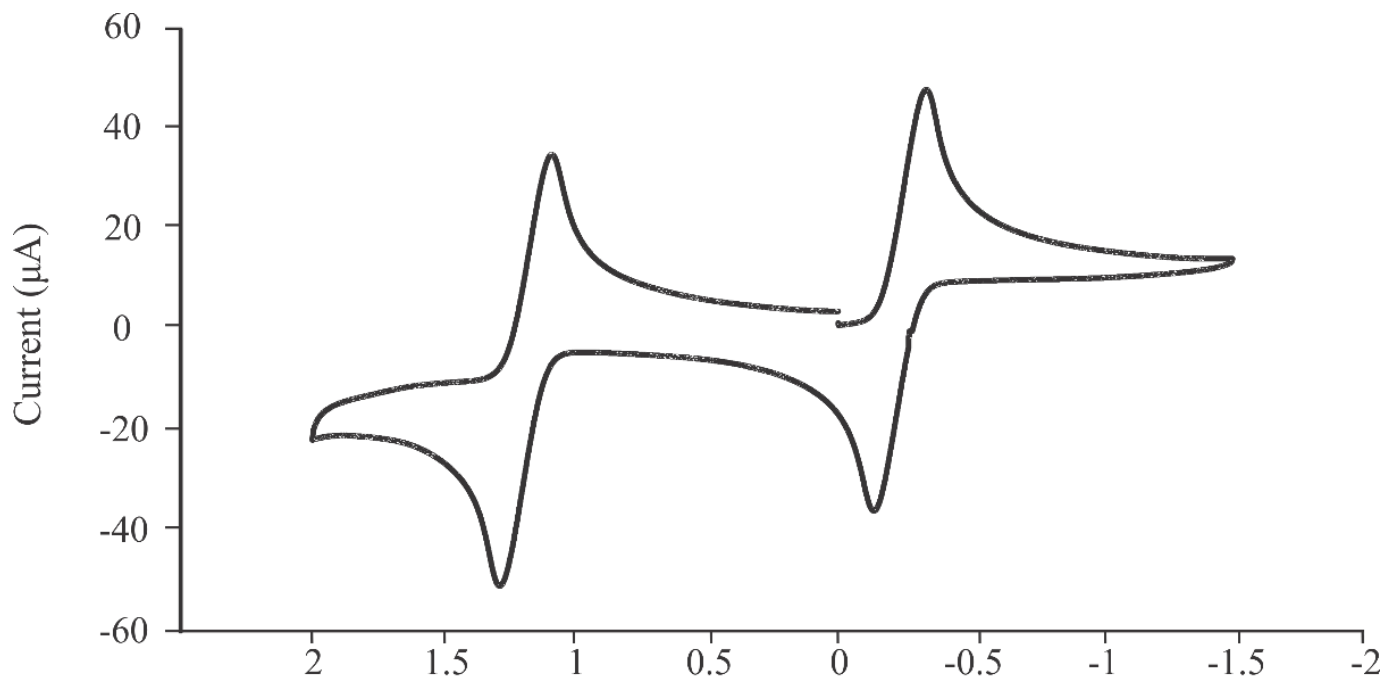

Potential vs $\mathrm{Fc}^{+} / \mathrm{Fc}^{0}(\mathrm{~V})$

Figure A34. Cyclic voltammogram of 15 in DCM at $200 \mathrm{mV} / \mathrm{s}$

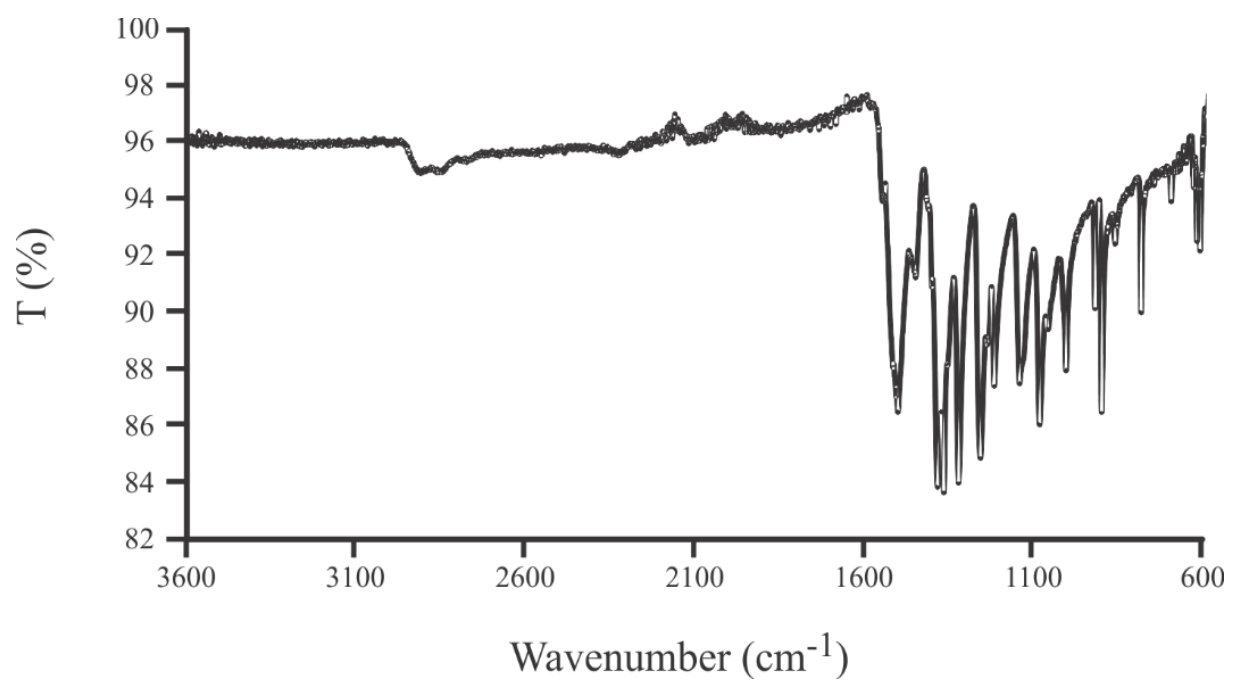

Figure A35. FTIR of $\mathbf{2 8}$ as a powder (ATR) 
Table A3. Parameters obtained by fitting frequency response analysis (FRA) data to simulated RCW circuit

Zview software ${ }^{179}$ was used to fit data. $Q$ is the magnitude of the CPE, $R_{c}$ is the charge transfer resistance, $\mathrm{R}_{\mathrm{S}}$ is approximate solution resistance, $\mathrm{n}$ is the CPE exponent, and $\mathrm{C}$ is the capacitance. Capacitance values were calculated using $C=R_{C}^{\frac{1-n}{n}} * Q^{\frac{1}{n}} \cdot{ }^{180-184}$

\begin{tabular}{l|lll|lll|lll}
$\mathbf{G C}$ & $\mathbf{1}$ & & & $\mathbf{2}$ & & & $\mathbf{3}$ & & \\
$\#$ & $\mathbf{0}$ & $\mathbf{3 0 0}$ & $\mathbf{1 0 0 0}$ & $\mathbf{0}$ & $\mathbf{2 0 0}$ & $\mathbf{1 0 0 0}$ & $\mathbf{0}$ & $\mathbf{1 0 0 0}$ & $\mathbf{1 5 0 0}$ \\
$\mathbf{C y c l e}$ & & & & & & & & & \\
$\mathbf{S}$ & & & & & & & & & \\
$\mathbf{Q}(\mathbf{F}$ & $2.43 \mathrm{E}-$ & $4.52 \mathrm{E}-$ & $4.46 \mathrm{E}-$ & $2.63 \mathrm{E}-$ & $6.10 \mathrm{E}-$ & $6.33 \mathrm{E}-$ & $2.24 \mathrm{E}-$ & $1.75 \mathrm{E}-$ & $1.73 \mathrm{E}-$ \\
$\mathbf{\mathbf { s } ^ { - 1 } \mathbf { 1 }}$ & 6 & 6 & 6 & 6 & 6 & 6 & 6 & 6 & 6 \\
$\mathbf{R}_{\mathbf{c}}$ & 25099 & 270 & 374 & 18843 & 158 & 374 & 34518 & 34049 & 45793 \\
$\mathbf{( \Omega )}$ & & & & & & & & & \\
$\mathbf{R}_{\mathbf{s}}$ & 26.22 & 20.12 & 17.99 & 16.80 & 15.09 & 13.9 & 7.24 & 5.37 & 5.78 \\
$\mathbf{( \Omega )}$ & & & & & & & & & \\
$\mathbf{N}$ & 0.908 & 0.929 & 0.913 & 0.916 & 0.893 & 0.864 & 0.893 & 0.899 & 0.896 \\
$\mathbf{C}(\mathbf{F})$ & $1.83 \mathrm{E}-$ & $2.71 \mathrm{E}-$ & $2.43 \mathrm{E}-$ & $2.00 \mathrm{E}-$ & $2.65 \mathrm{E}-$ & $2.49 \mathrm{E}-$ & $1.65 \mathrm{E}-$ & $1.27 \mathrm{E}-$ & $1.29 \mathrm{E}-$ \\
& 6 & 6 & 6 & 6 & 6 & 6 & 6 & 6 & 6 \\
$\boldsymbol{\chi}^{\mathbf{2}}$ & 0.0001 & 0.0012 & 0.0014 & 0.0007 & 0.0005 & 0.0005 & 0.0003 & 0.0031 & 0.0025 \\
& 5 & 1 & 1 & 2 & 7 & 7 & 0 & 3 & 2
\end{tabular}

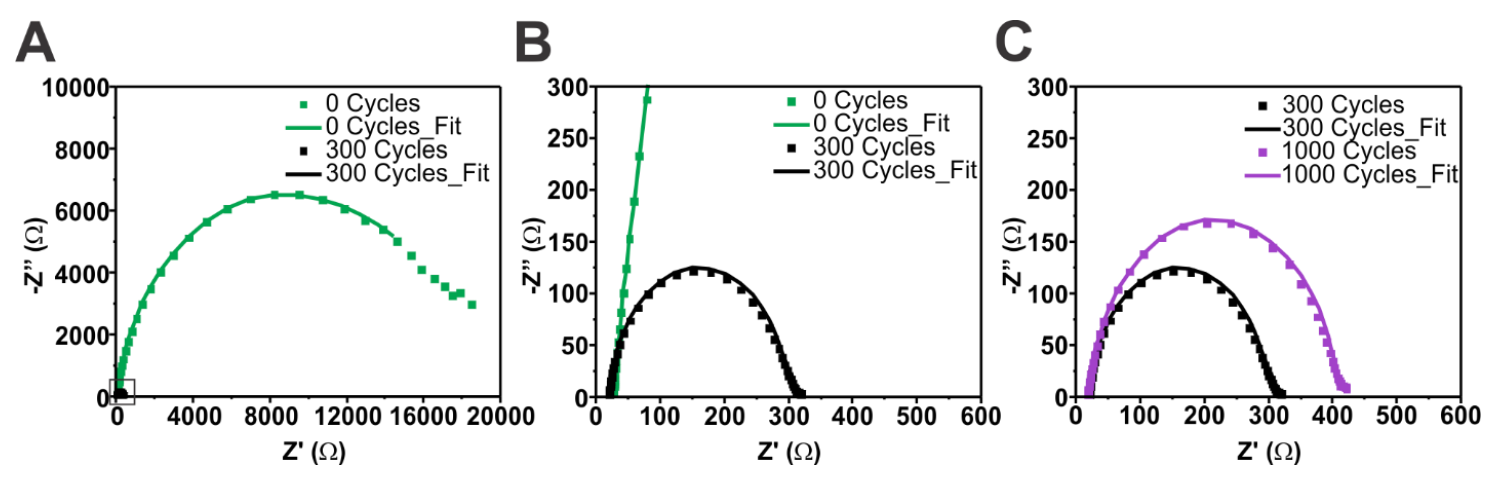

Figure A36. Nyquist plots showing the effects of reductive cycling from 0 to $-0.8 \mathrm{~V}$ vs. RHE at $50 \mathrm{mV} / \mathrm{sec}$ on the impedance of GC-1.

Solid traces represent fitted impedance data, while dotted traces represent actual data. (A) Nyquist plot showing impedance at 0 cycles (before any reductive cycling) and after 300 cycles (peak activity, in small frame). (B) The same data as in (A), but with axes scaled down to show impedance after 300 cycles in greater detail. (C) Comparison of Nyquist plots for GC-1 after 300 cycles and 1000 cycles. 


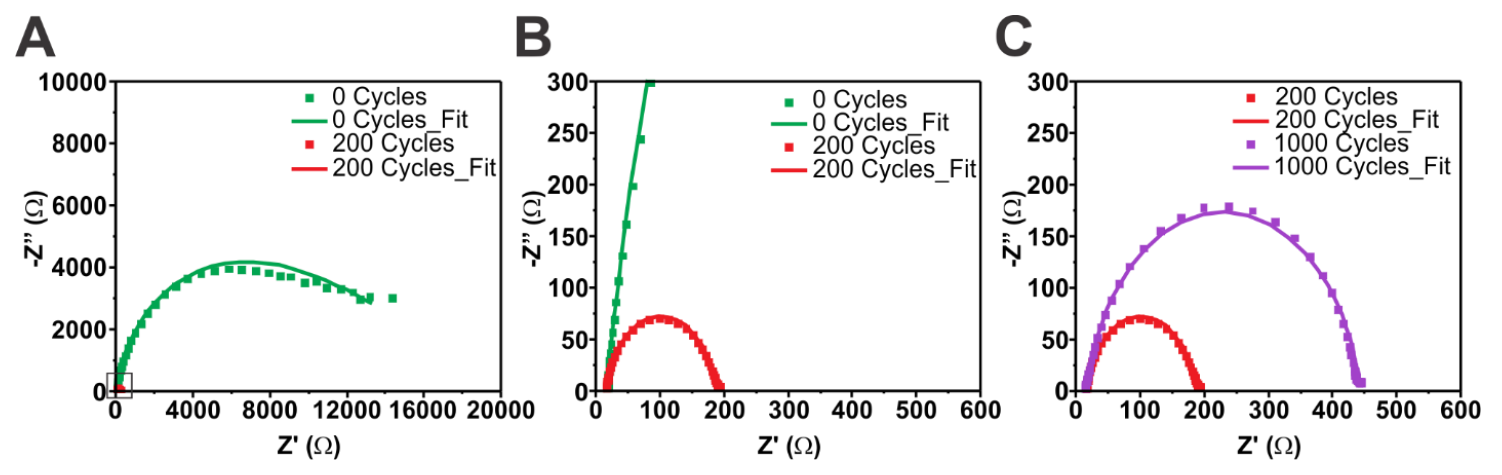

Figure A37. Nyquist plots showing the effects of reductive cycling from 0 to $-0.8 \mathrm{~V}$ vs. $\mathrm{RHE}$ at $50 \mathrm{mV} / \mathrm{sec}$ on the impedance of GC-2.

Solid traces represent fitted impedance data, while dotted traces represent actual data. (A) Nyquist plot showing impedance at 0 cycles (before any reductive cycling) and after 200 cycles (peak activity, in small frame). (B) The same data as in (A), but with axes scaled down to show impedance after 200 cycles in greater detail. (C) Comparison of Nyquist plots for GC-2 after 200 and 1000 cycles.
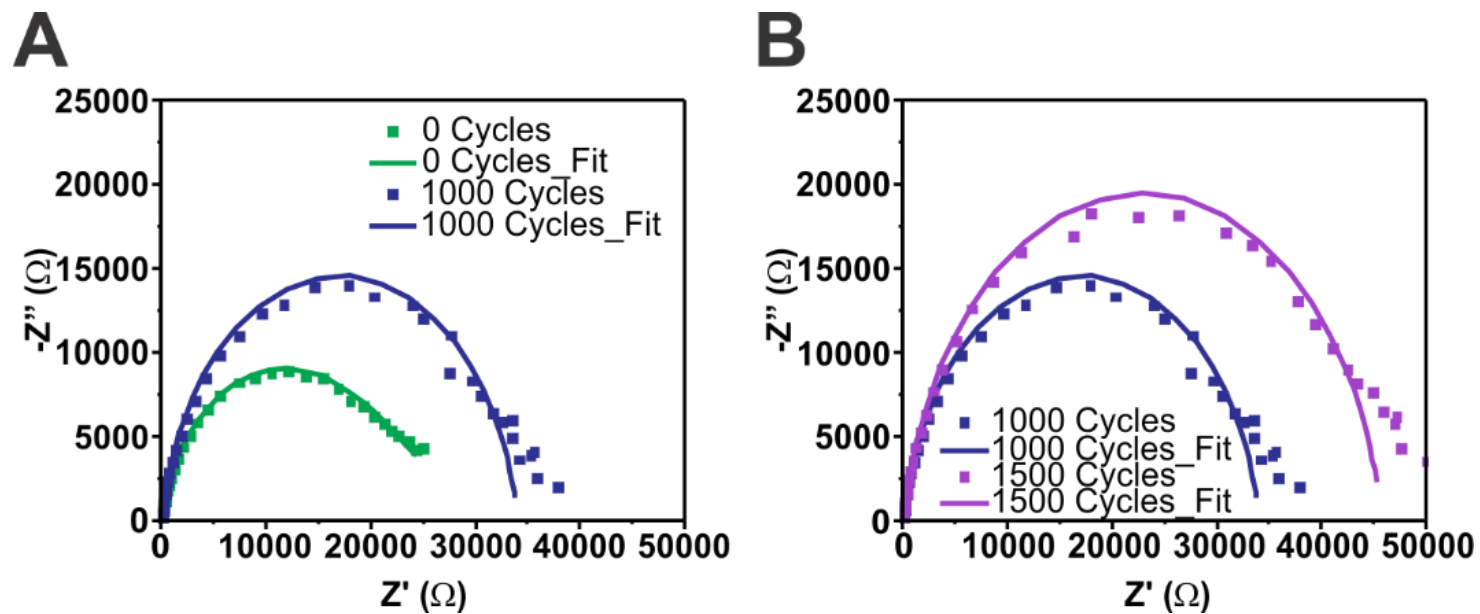

Figure A38. Nyquist plots showing the effects of cycling from 0 to $-0.8 \mathrm{~V}$ vs. RHE at 50 $\mathrm{mV} / \mathrm{sec}$ on the impedance of GC-3.

Solid traces represent fitted impedance data, while dotted traces represent actual data. (A) Comparison of Nyquist plots for GC-3 at 0 cycles (before any reductive cycling) and after 1000 cycles. (B) Comparison of Nyquist plots for GC-3 after 1000 and 1500 cycles. 


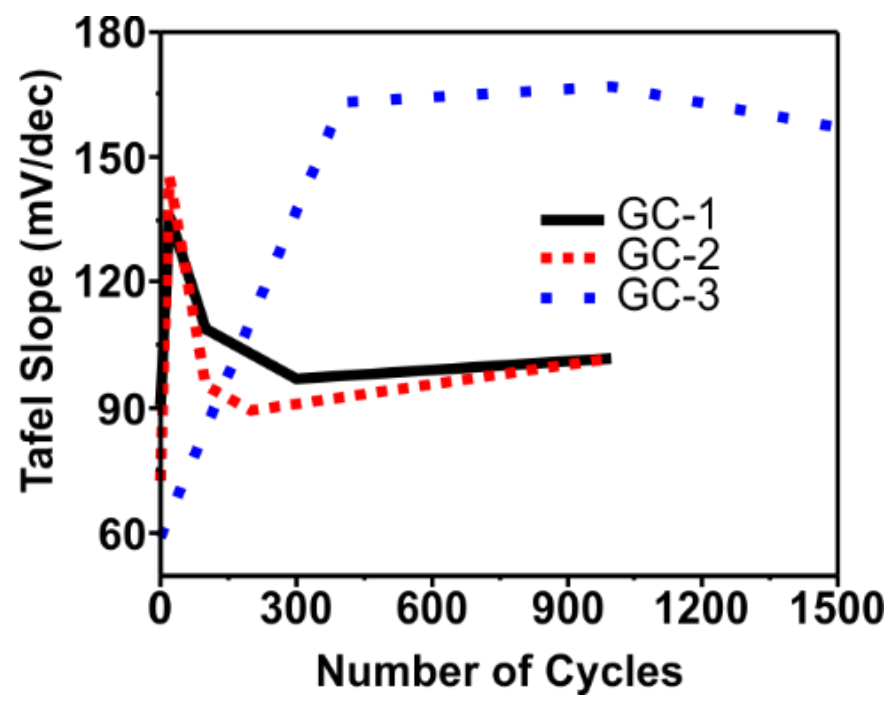

Figure A39. Tafel slope as a function of number of cycles for each modified glassy carbon electrode.

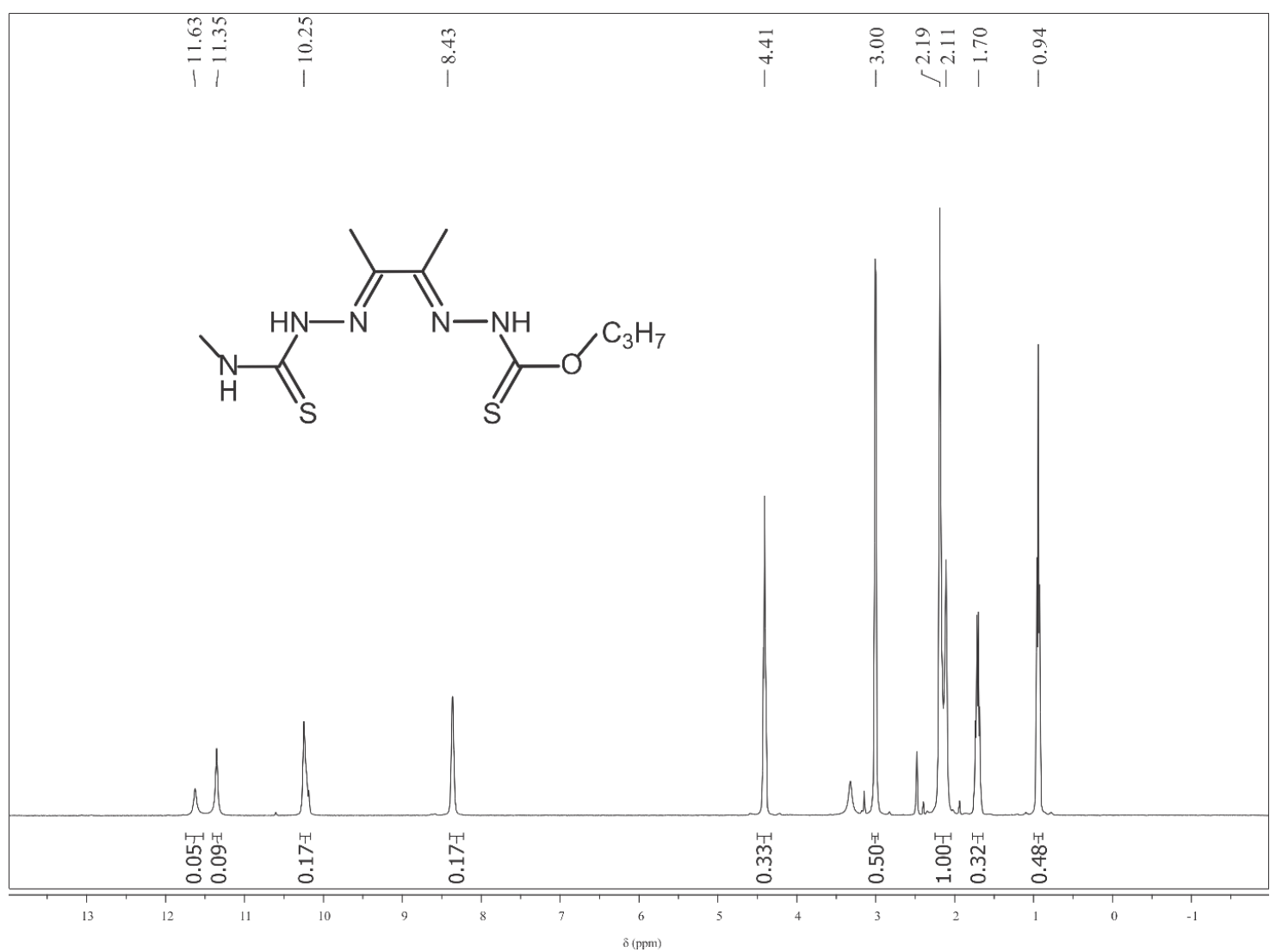

Figure A40. ${ }^{1} \mathrm{H}$ NMR $(400 \mathrm{MHz})$ of 17 in DMSO-D 6 .

$\delta 0.94(\mathrm{br}, 3 \mathrm{H}) 1.70(\mathrm{br}, 2 \mathrm{H}), 2.11$ and $2.19(\mathrm{~s} / \mathrm{br}, 6 \mathrm{H}) 3.00(\mathrm{~s}, 3 \mathrm{H}) 4.41$ (br, 2H) 8.43 $(\mathrm{s}, 1 \mathrm{H}) 10.25(\mathrm{~s}, 1 \mathrm{H}) 11.35$ and $11.63(\mathrm{~s}, 1 \mathrm{H})$ 


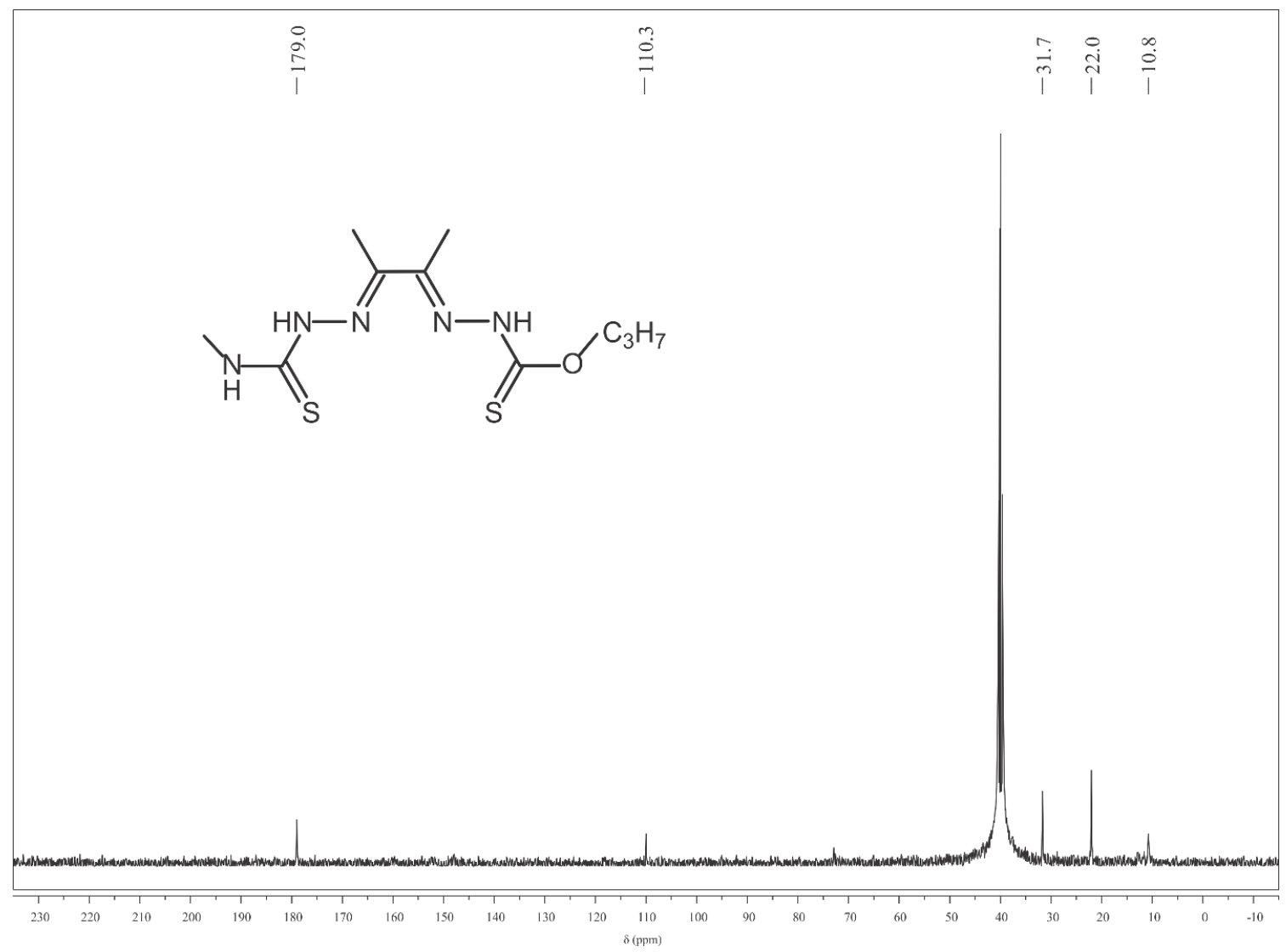

Figure A41. ${ }^{13} \mathrm{C}$ NMR (100 MHz) of 17 in DMSO-d 6 .

$\delta 10.8,22.0,31.7,179.0$ 


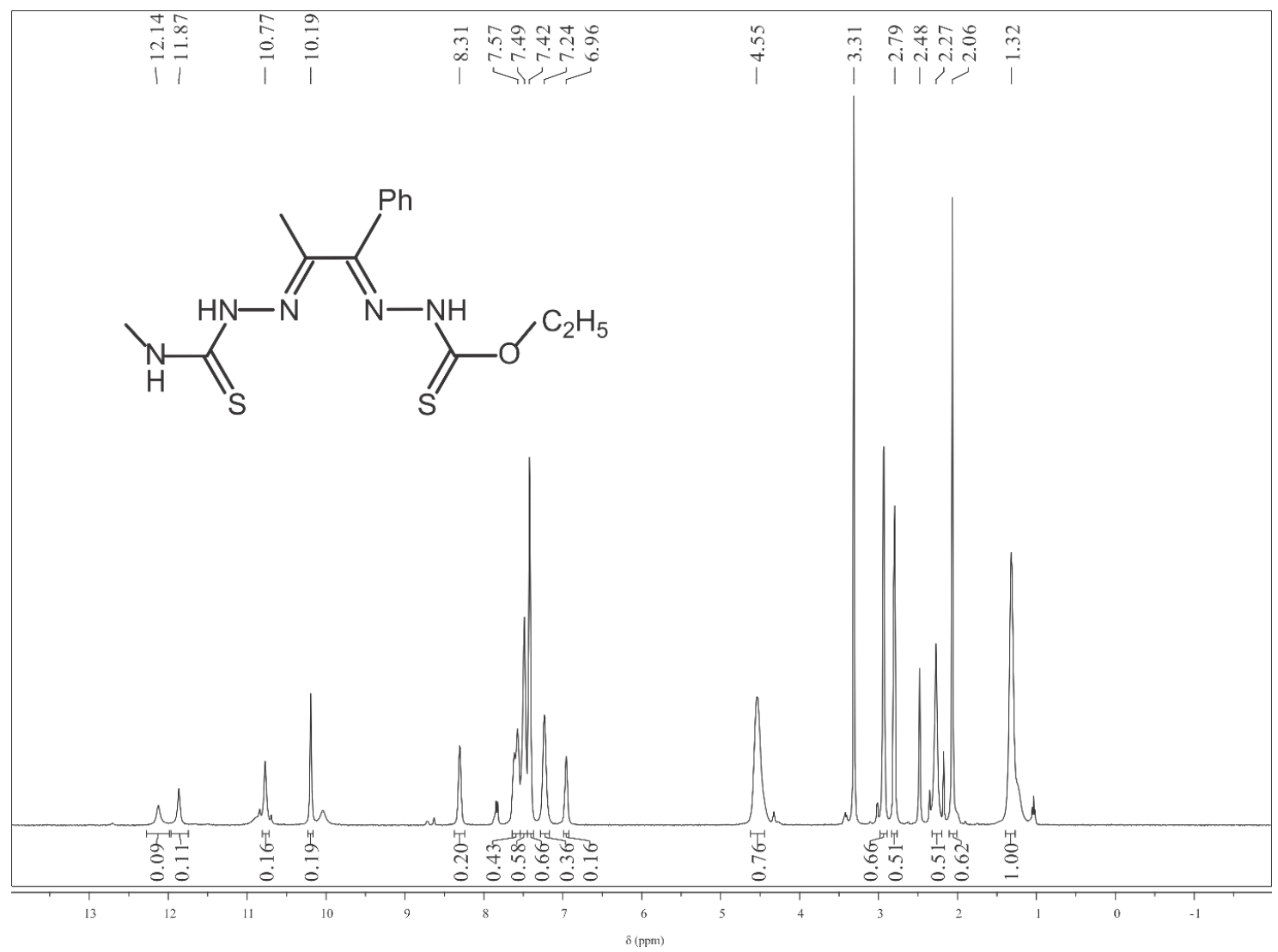

Figure A42. ${ }^{1} \mathrm{H}$ NMR (400 MHz) of 18 in DMSO-d 6 .

Contaminated with starting material, as evidenced by peaks at 2.93 and 2.06 


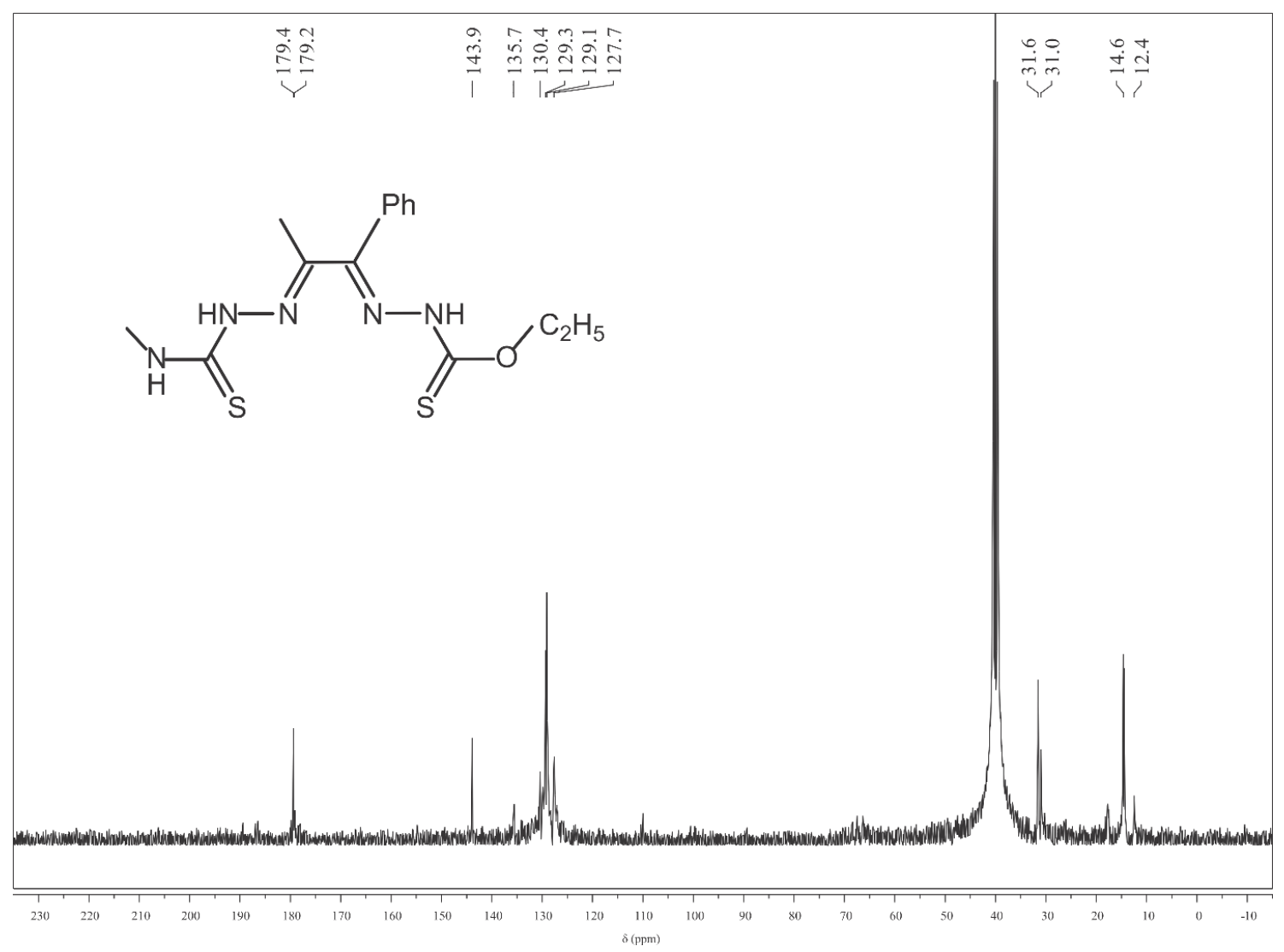

Figure A43. ${ }^{13} \mathrm{C}$ NMR (100 MHz) of 18 in DMSO-d 6 .

Contaminated with starting material.

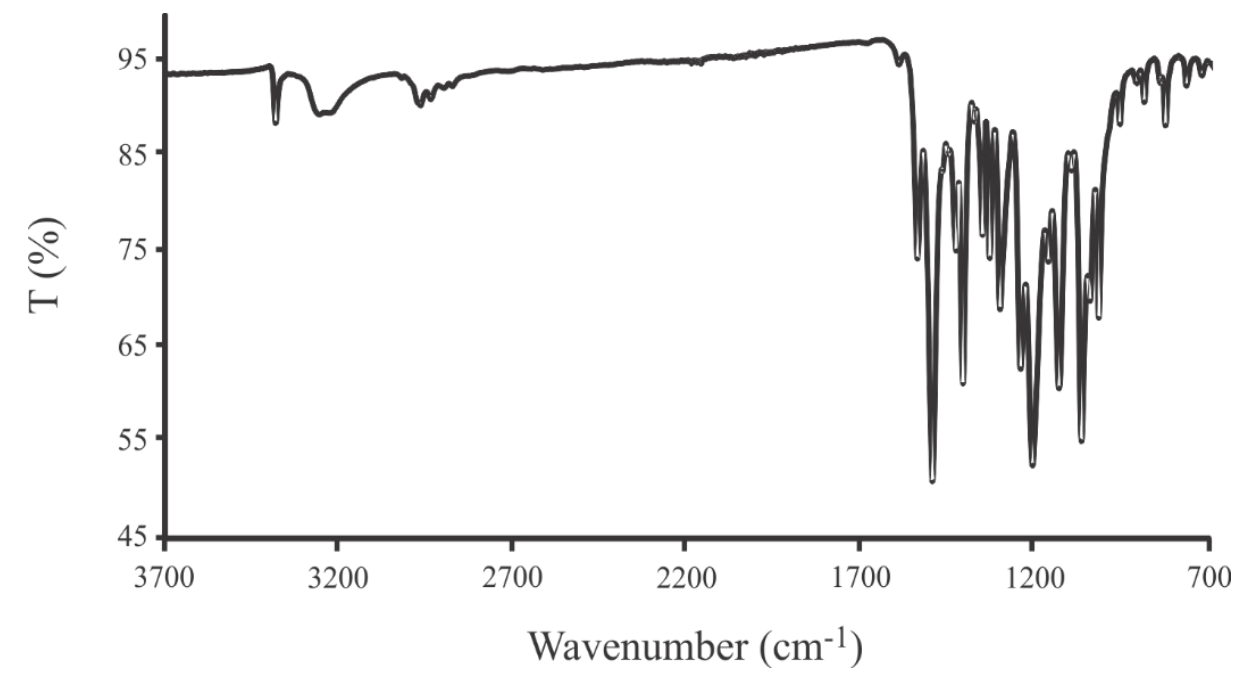

Figure A44. FT-IR of 17 as a powder (ATR) 


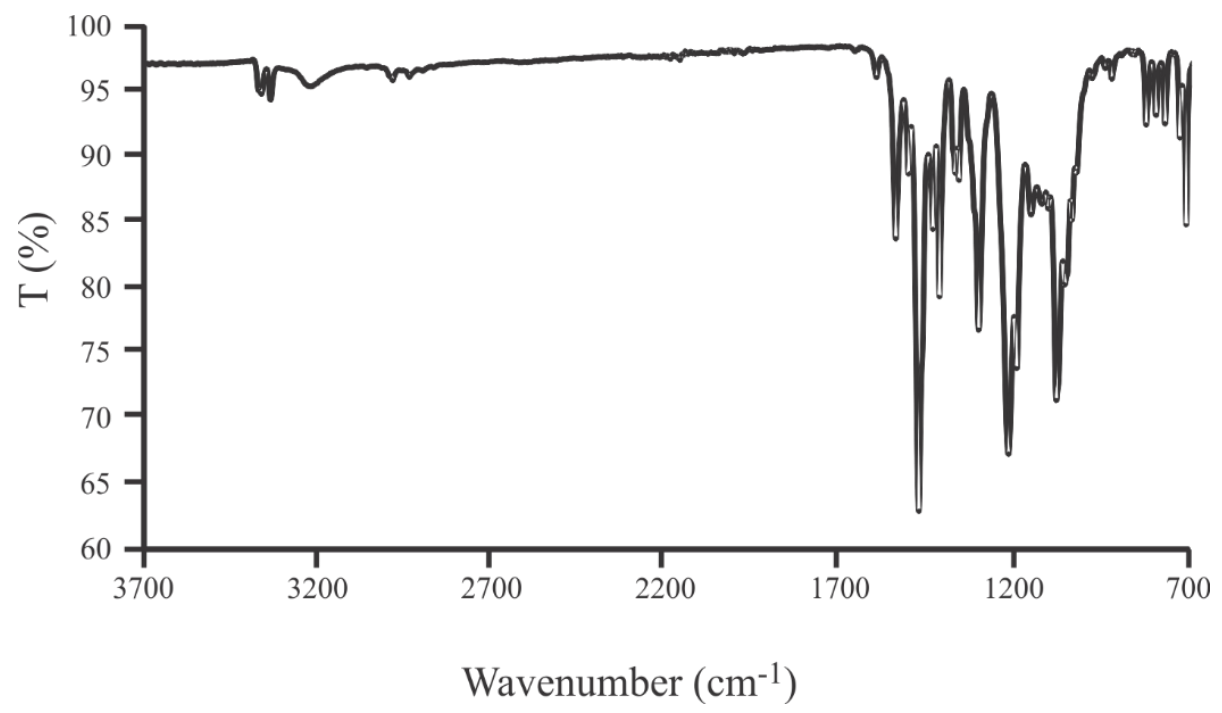

Figure A45. FT-IR of 18 as a powder (ATR)

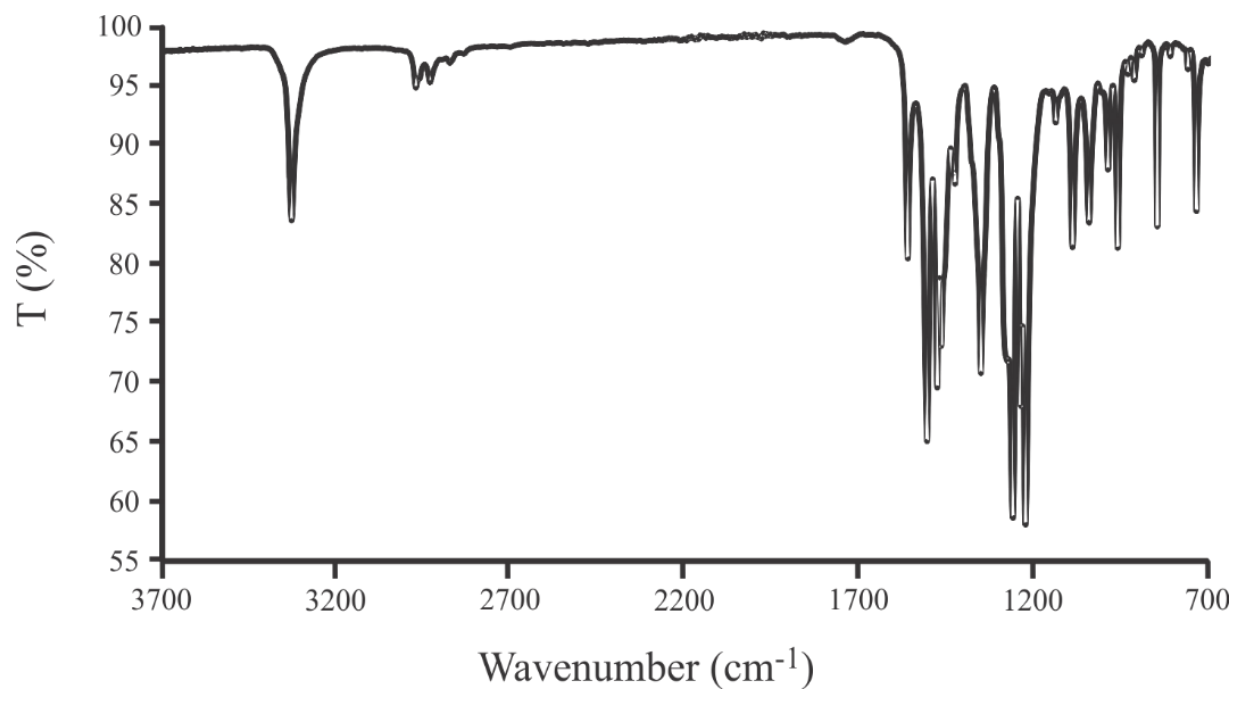

Figure A46. FT-IR of 20 as a powder (ATR) 


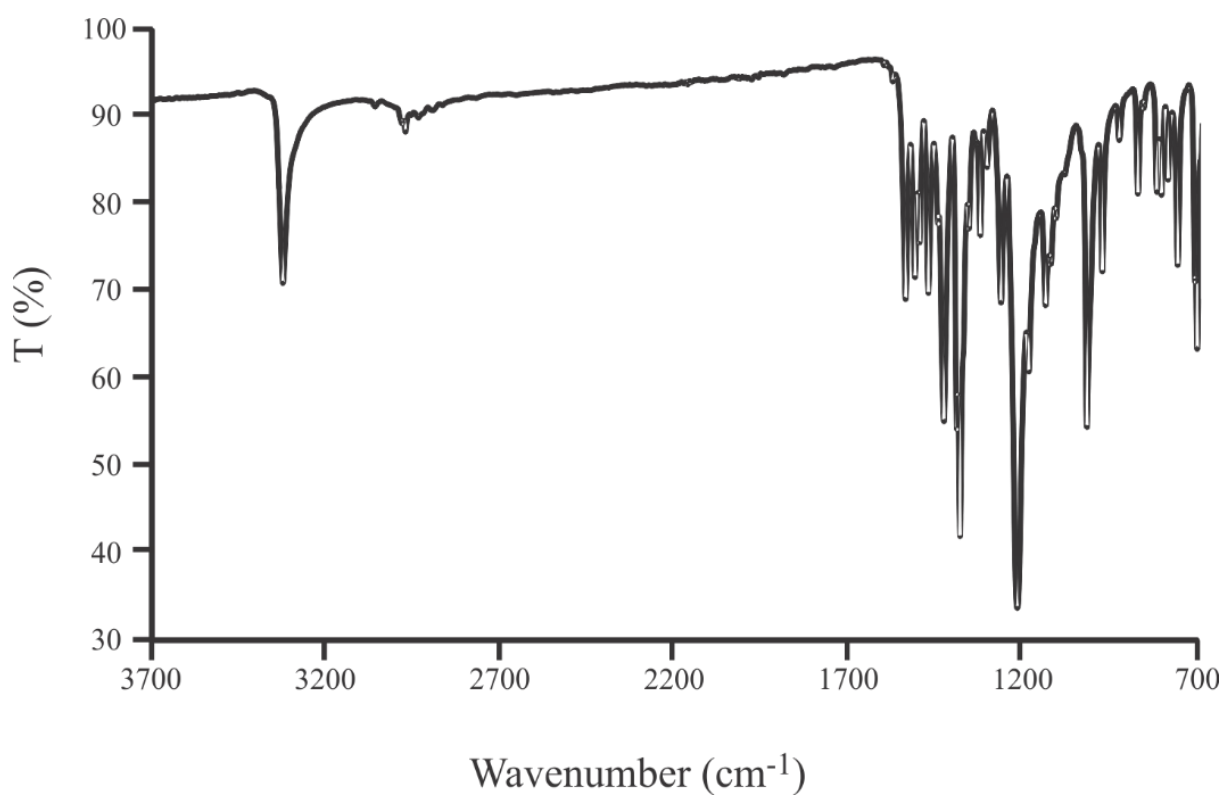

Figure A46. FT-IR of 21 as a powder (ATR)

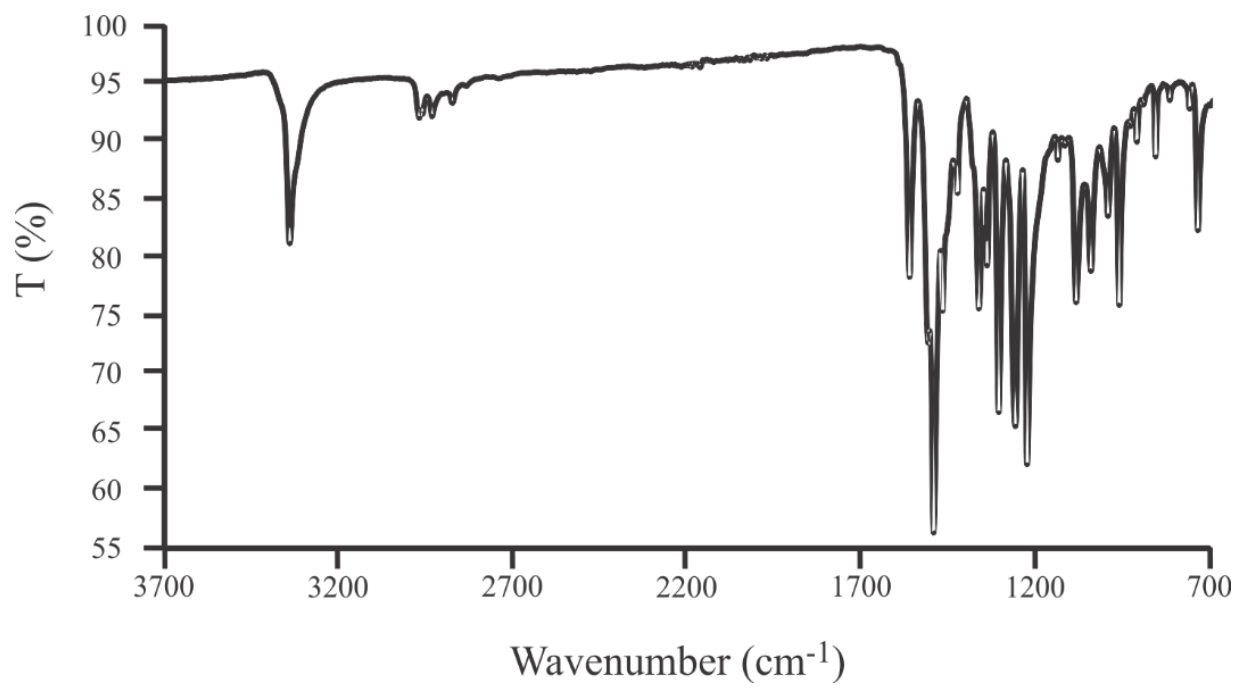

Figure A47. FT-IR of 23 as a powder (ATR) 


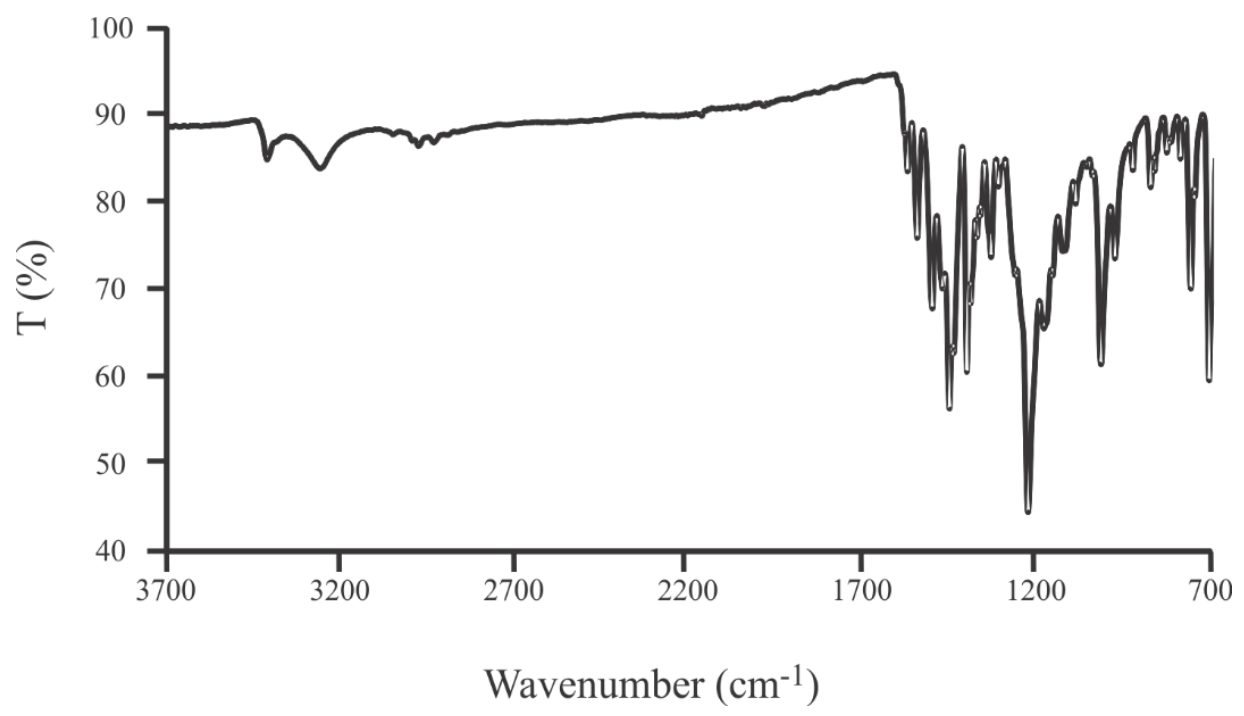

Figure A48. FT-IR of 24 as a powder (ATR)

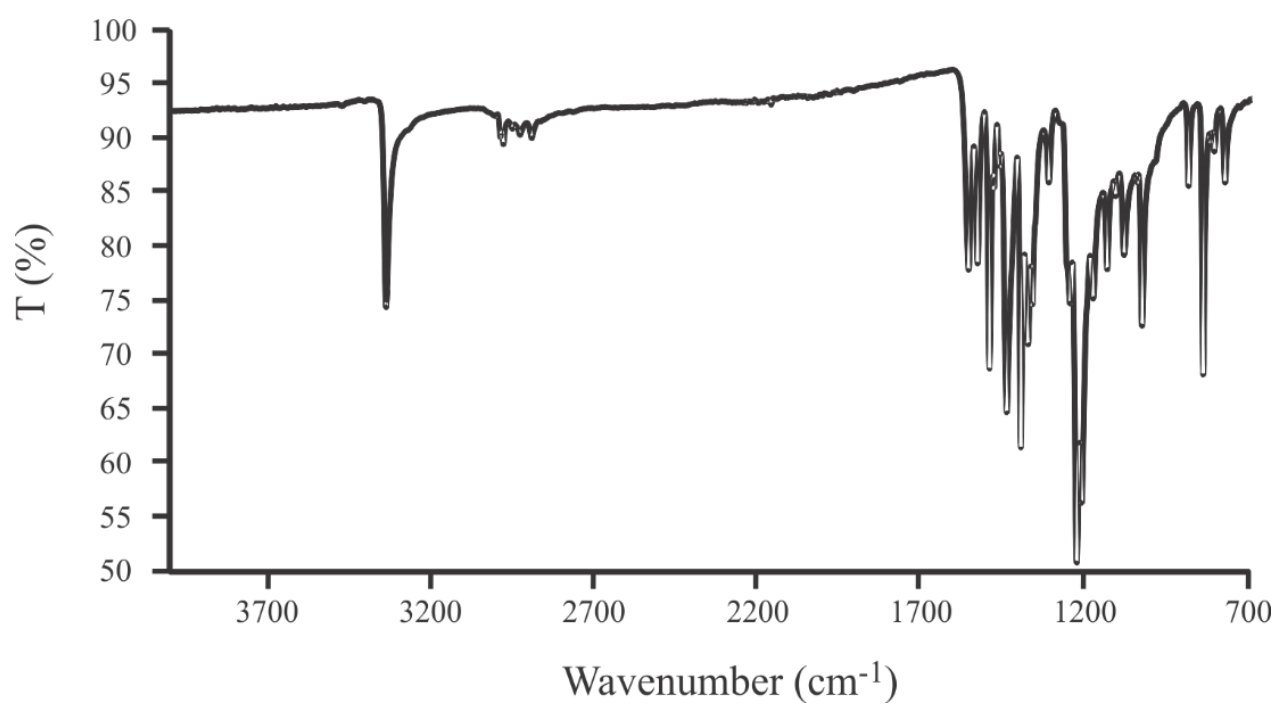

Figure A49. FT-IR of 25 as a powder (ATR) 


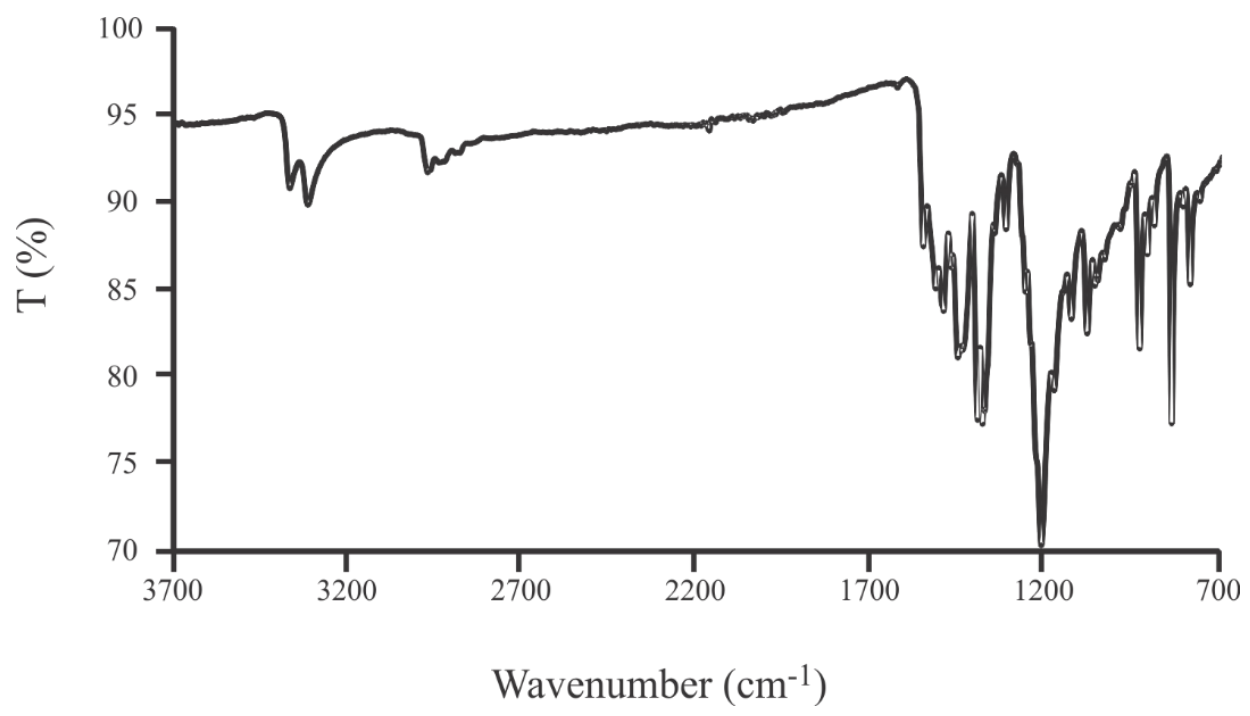

Figure A50. FT-IR of 26 as a powder (ATR)

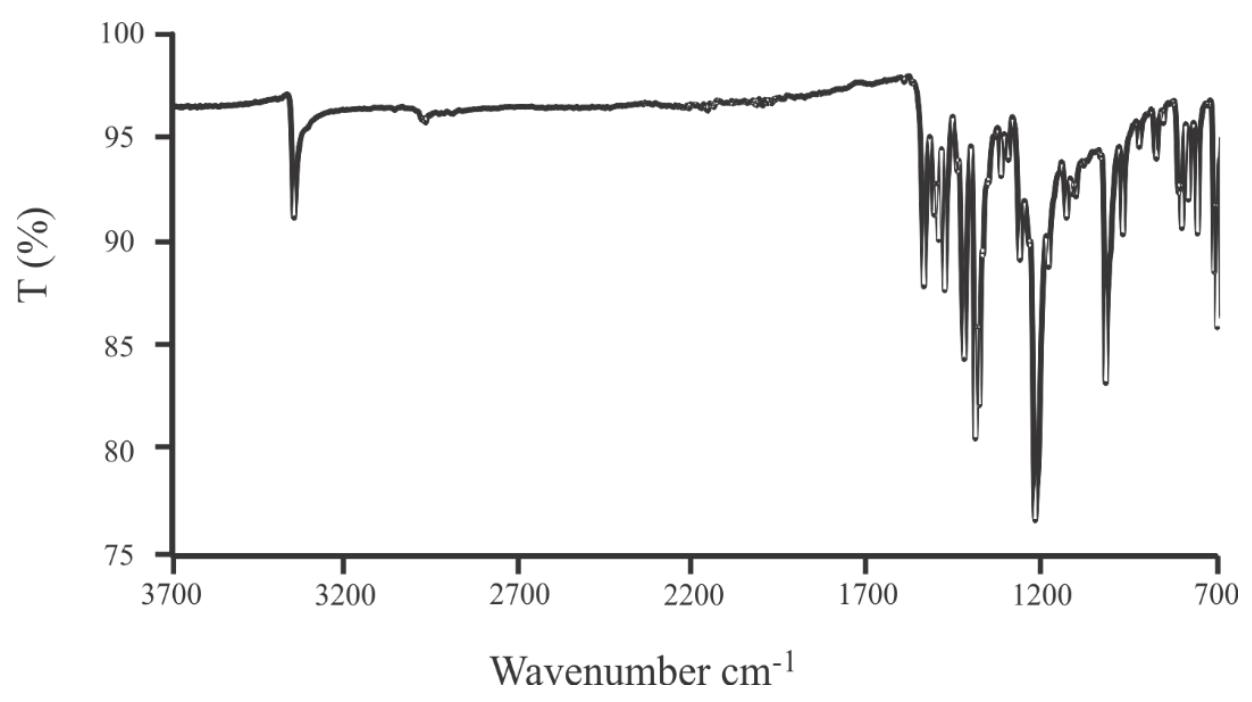

Figure A51. FT-IR of 27 as a powder (ATR) 


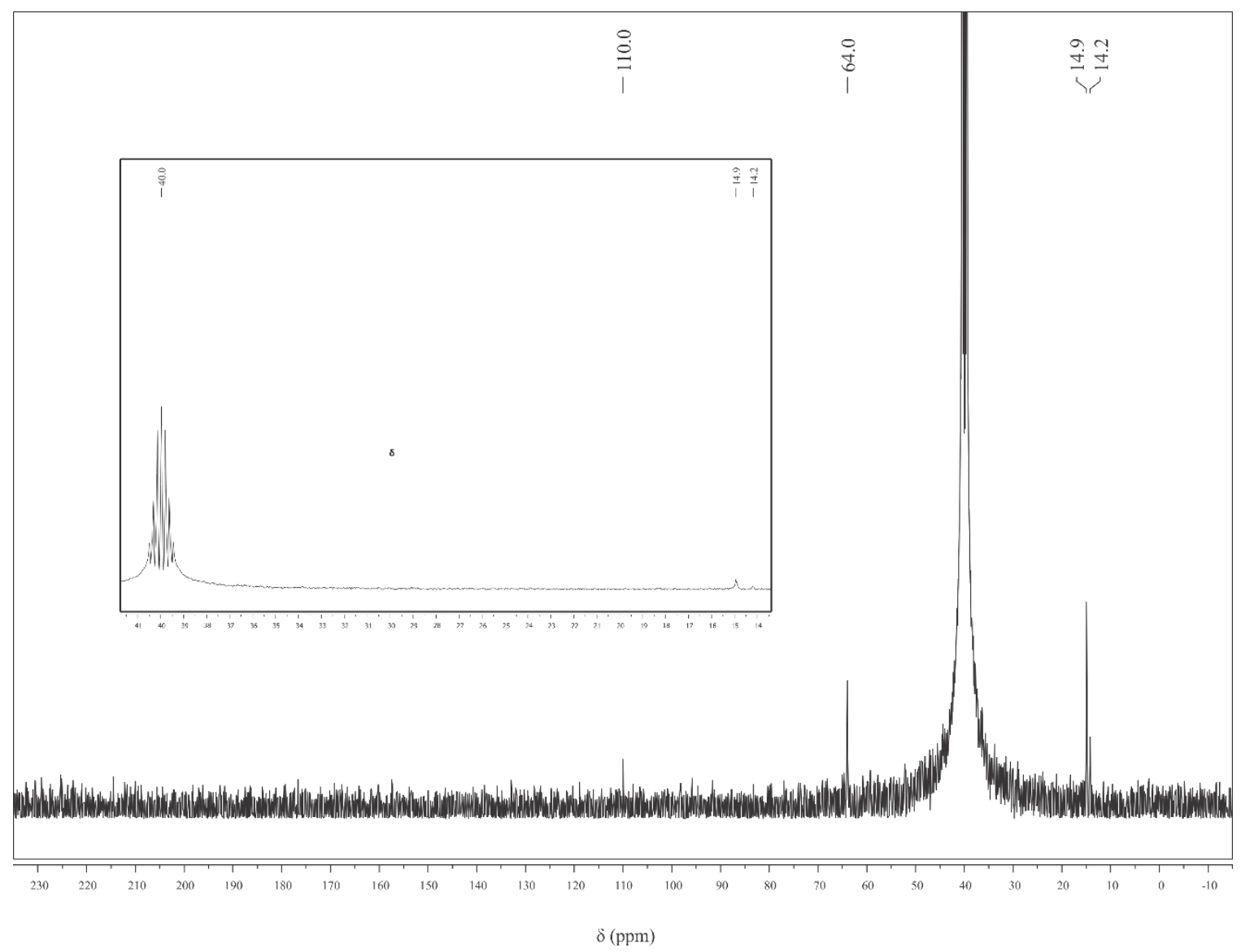

Figure A52. ${ }^{13} \mathrm{C}$ NMR (100 MHz) of 25 in DMSO-d 6 , inset shows DMSO.

$\delta 14.2,14.9,64.0,110.0$ 


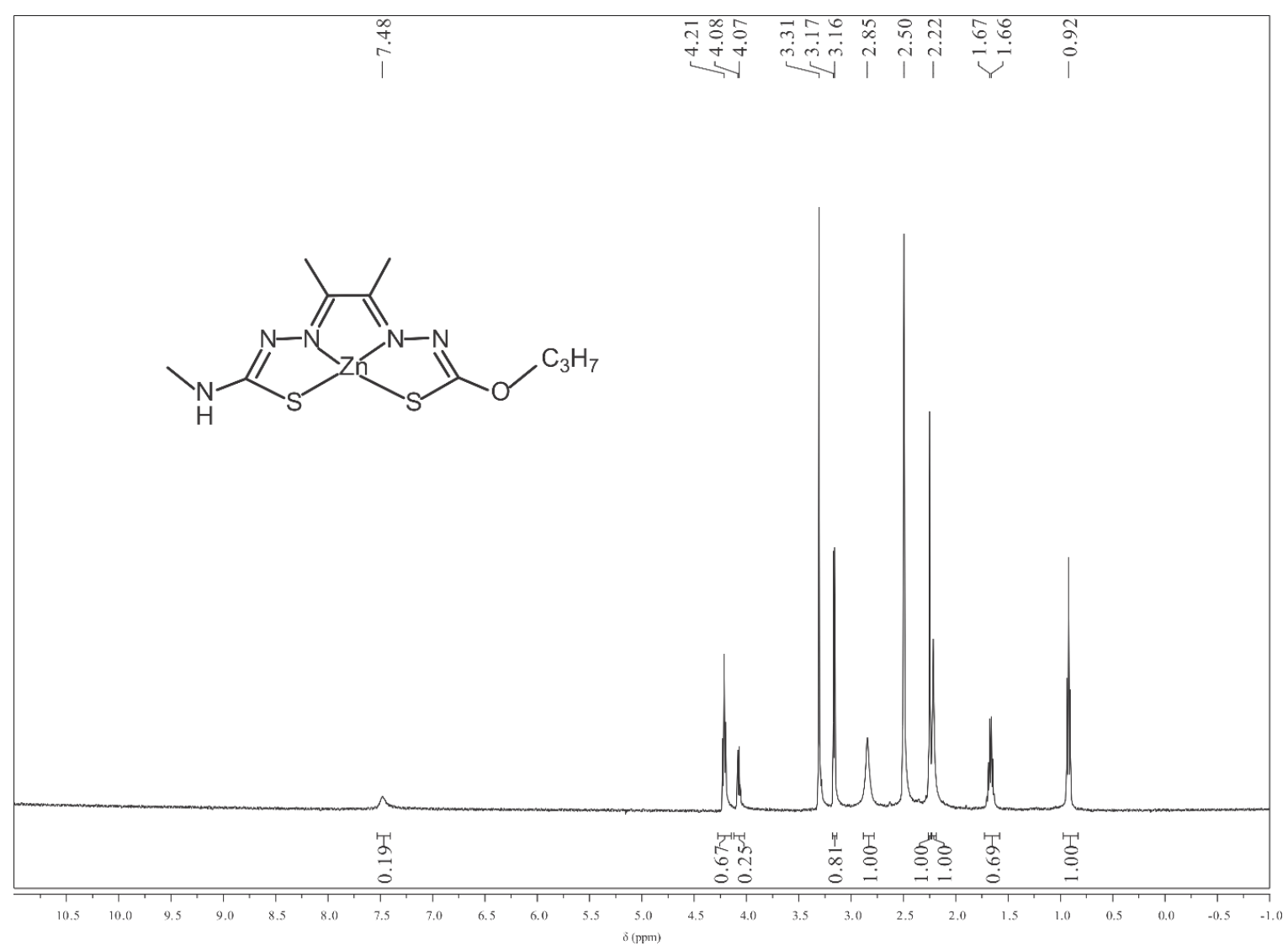

Figure A53. ${ }^{1} \mathrm{H}$ NMR $(400 \mathrm{MHz})$ of 26 in DMSO-d 6 .

$\delta 0.92(\mathrm{t}, 3 \mathrm{H}) 1.66(\mathrm{br}, 2 \mathrm{H}) 2.20(\mathrm{~s}, 3 \mathrm{H}) 2.22(\mathrm{~s}, 3 \mathrm{H}) 2.85(\mathrm{~s}, 3 \mathrm{H}) 4.21(\mathrm{br}, 2 \mathrm{H}) 7.48(\mathrm{~s}, 1 \mathrm{H})$. Methanol present, peaks at 3.16 and 4.08 . 


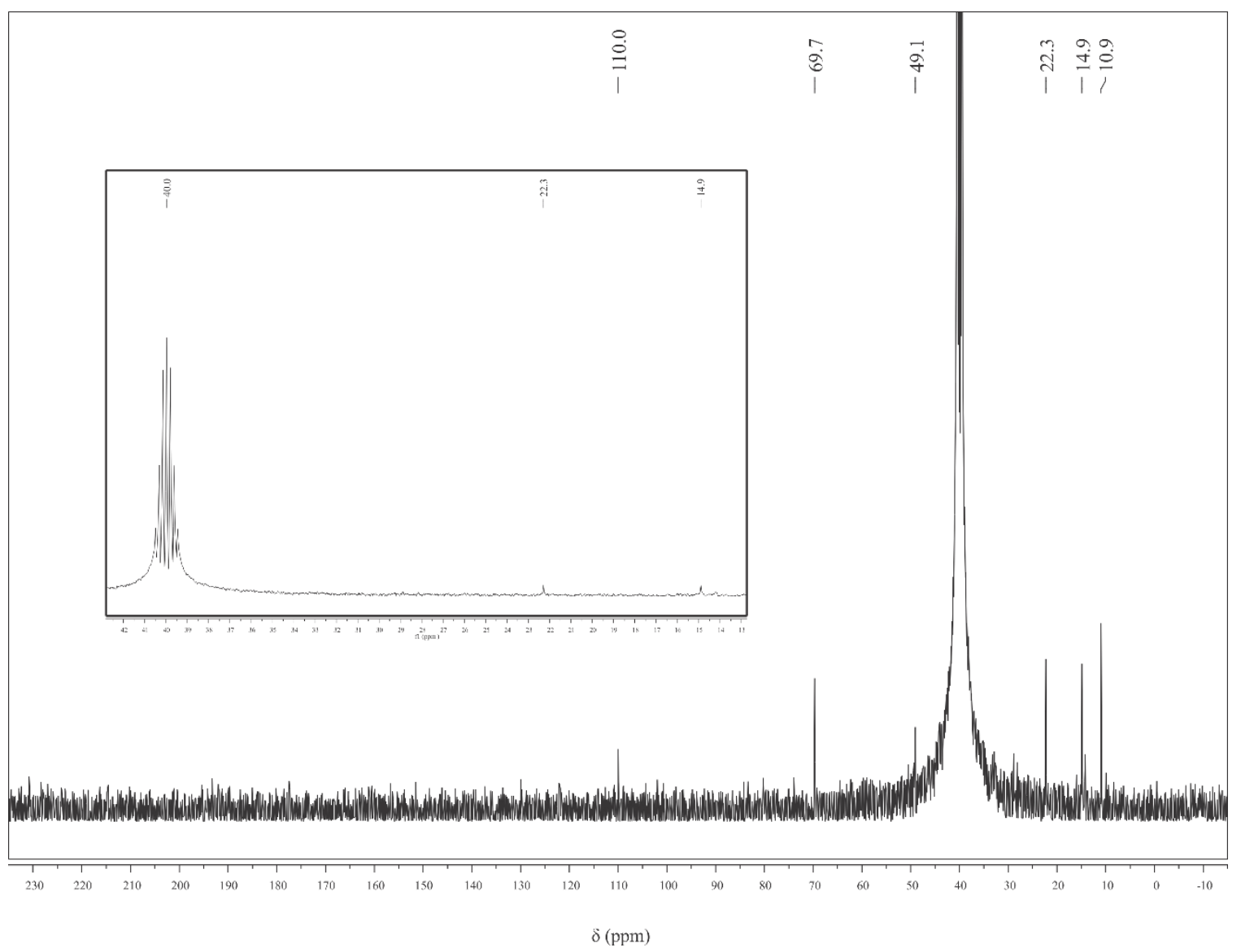

Figure A54. ${ }^{13} \mathrm{C}$ NMR(100 MHz) of 26 in DMSO-d , inset displays the DMSO. $\delta 10.9,14.9,22.3,69.7,110.0$ 


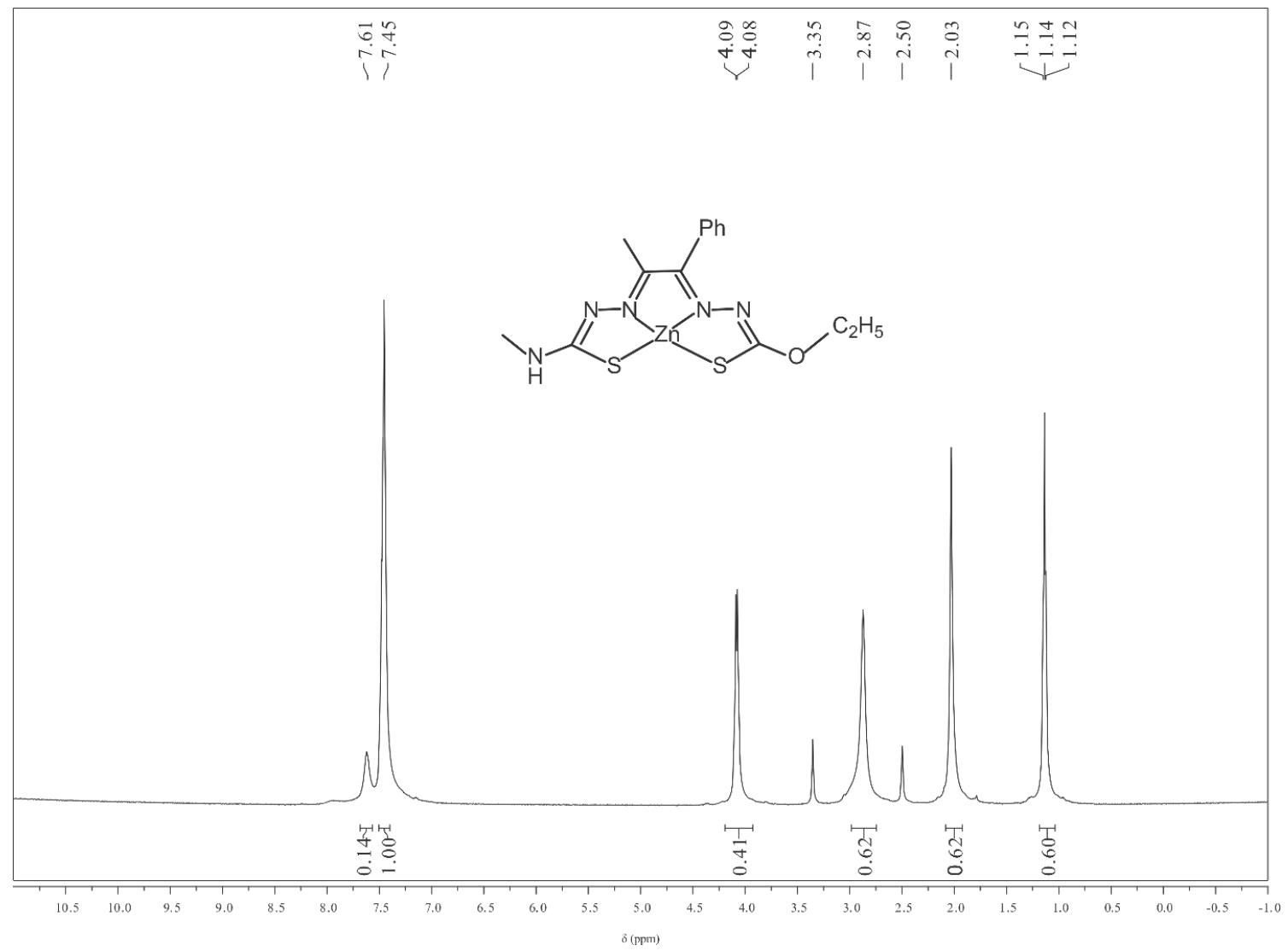

Figure A55. ${ }^{1} \mathrm{H}$ NMR (400MHz) of 27 in DMSO-d 6 .

$\delta 1.14(\mathrm{t}, 3 \mathrm{H}) 2.03(\mathrm{~s}, 3 \mathrm{H}) 2.87(\mathrm{~s}, 3 \mathrm{H}) 4.08(\mathrm{br}, 2 \mathrm{H}) 7.45(\mathrm{br}, 5 \mathrm{H}) 7.61(\mathrm{~s}, 1 \mathrm{H})$ 


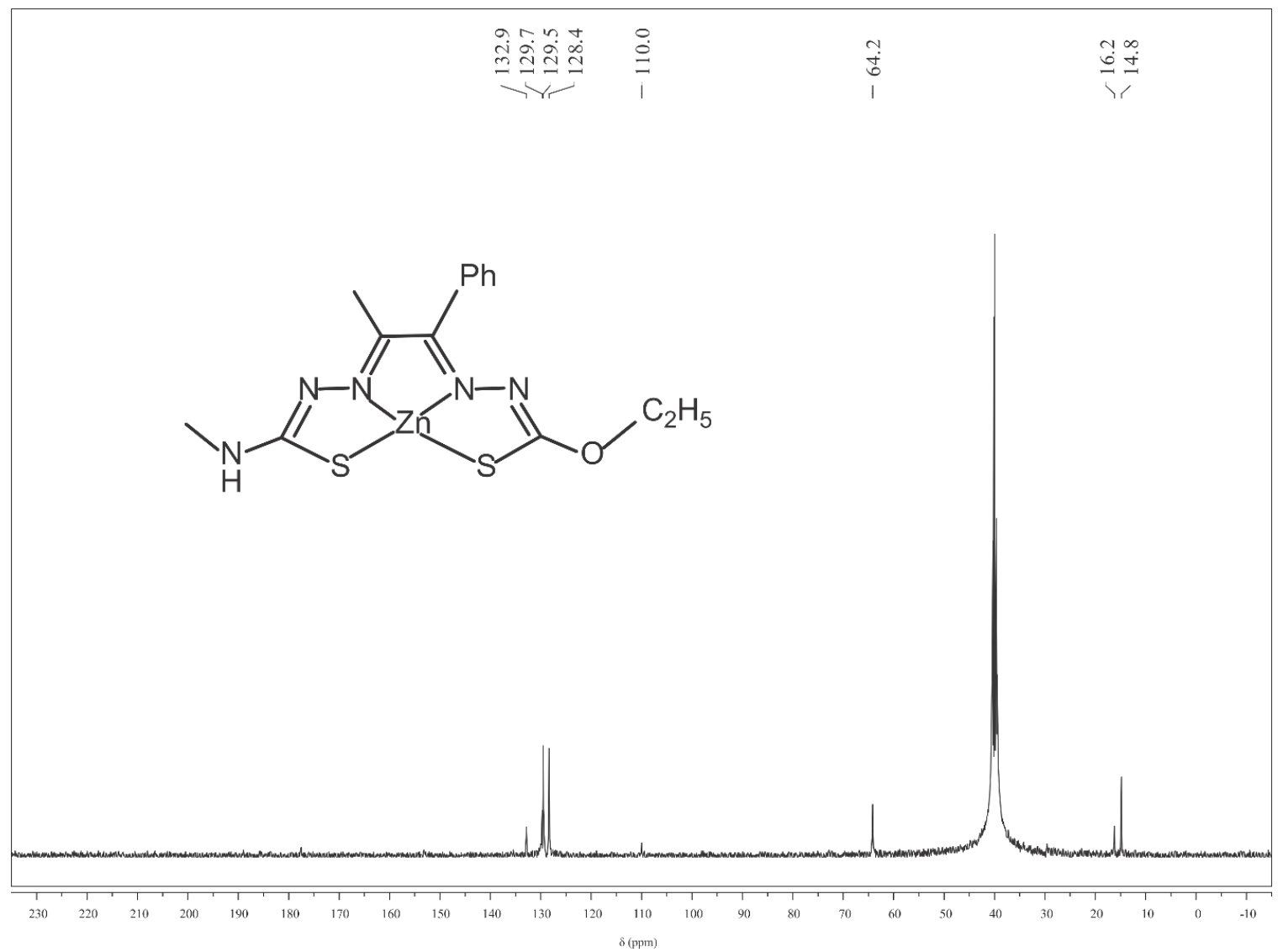

Figure A56. ${ }^{13} \mathrm{C}$ NMR $(100 \mathrm{MHz})$ of 27 in DMSO-d 6 .

$\delta 14.8,16.2,110.0,128.4,129.5,129.7,132.9$ 


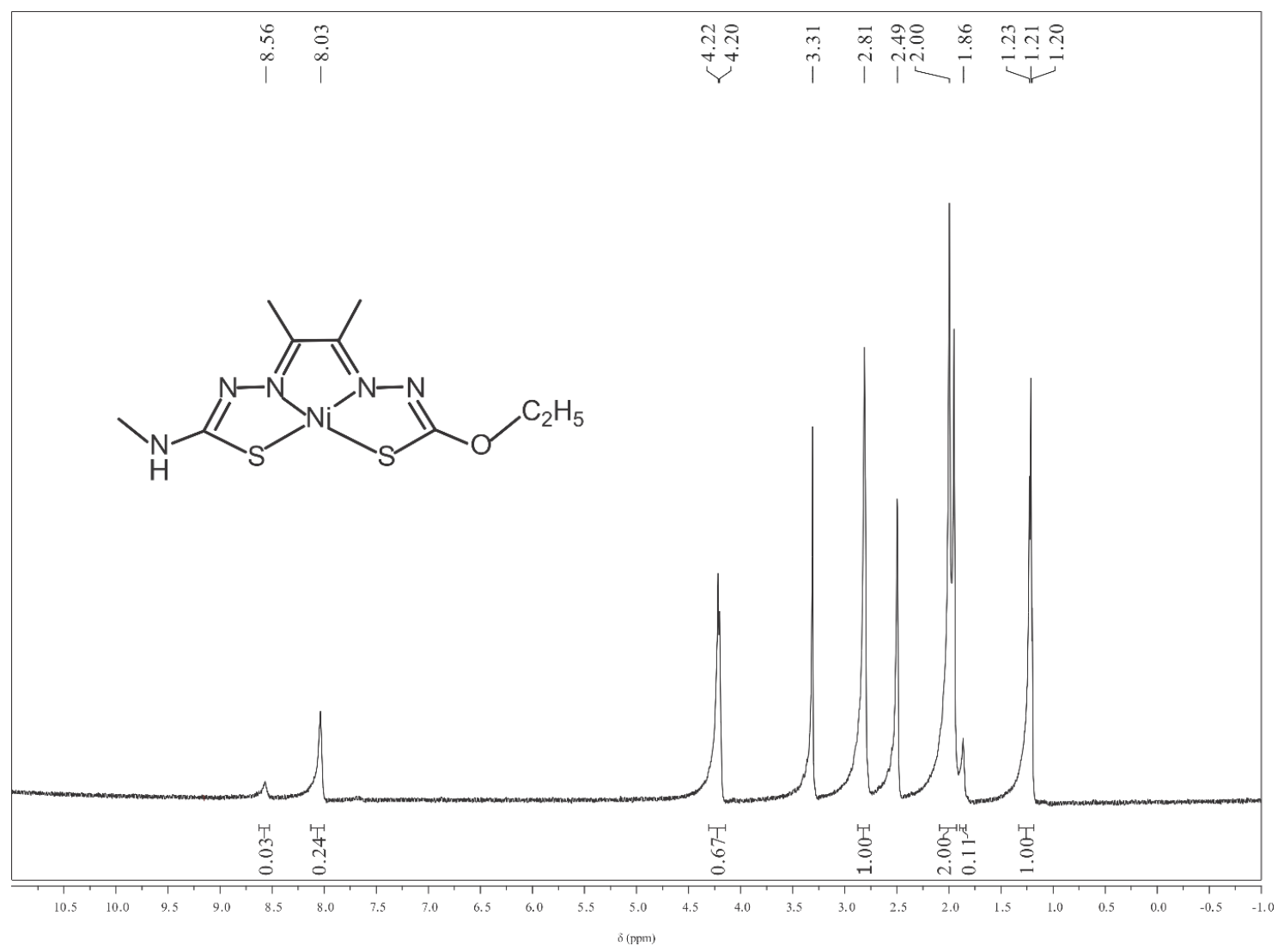

Figure A57. ${ }^{1} \mathrm{H}$ NMR (400 MHz) of 22 in DMSO-d 6 .

$\delta 1.21(\mathrm{t}, 3 \mathrm{H}) 2.00(\mathrm{~s}, 6 \mathrm{H}) 2.81(\mathrm{~s}, 3 \mathrm{H}) 4.20(\mathrm{br}, 2 \mathrm{H}) 8.03(\mathrm{~s}, 1 \mathrm{H})$ 


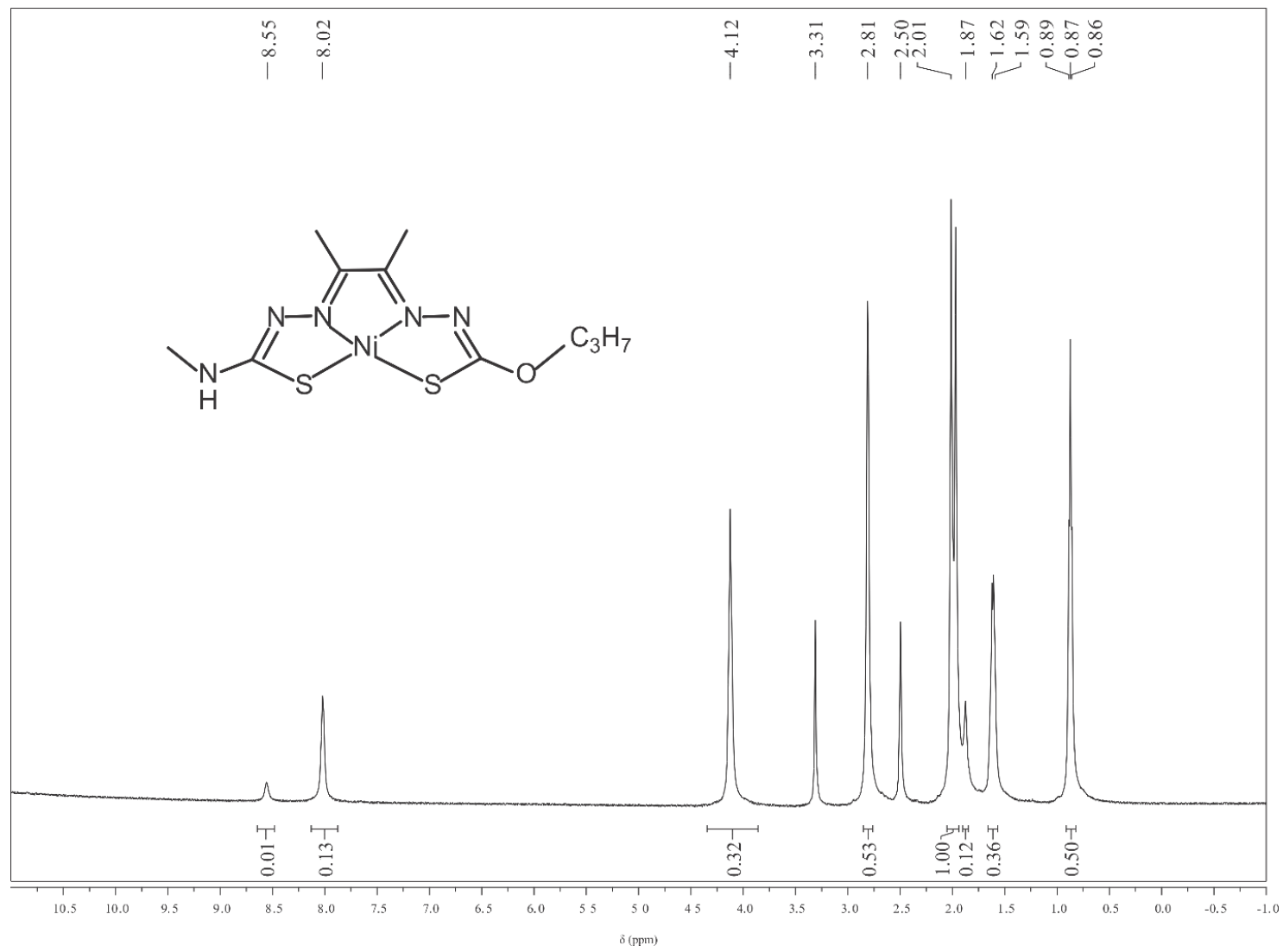

Figure A58. ${ }^{1} \mathrm{H}$ NMR (400 MHz) of 23 in DMSO-d . $_{6}$

$\delta 0.87(\mathrm{t}, 3 \mathrm{H}) 1.59(\mathrm{br}, 2 \mathrm{H}) 2.01(\mathrm{br}, 6 \mathrm{H}) 2.81(\mathrm{~s}, 3 \mathrm{H}) 4.12(\mathrm{br}, 2 \mathrm{H}) 8.02(\mathrm{~s}, 1 \mathrm{H})$ 


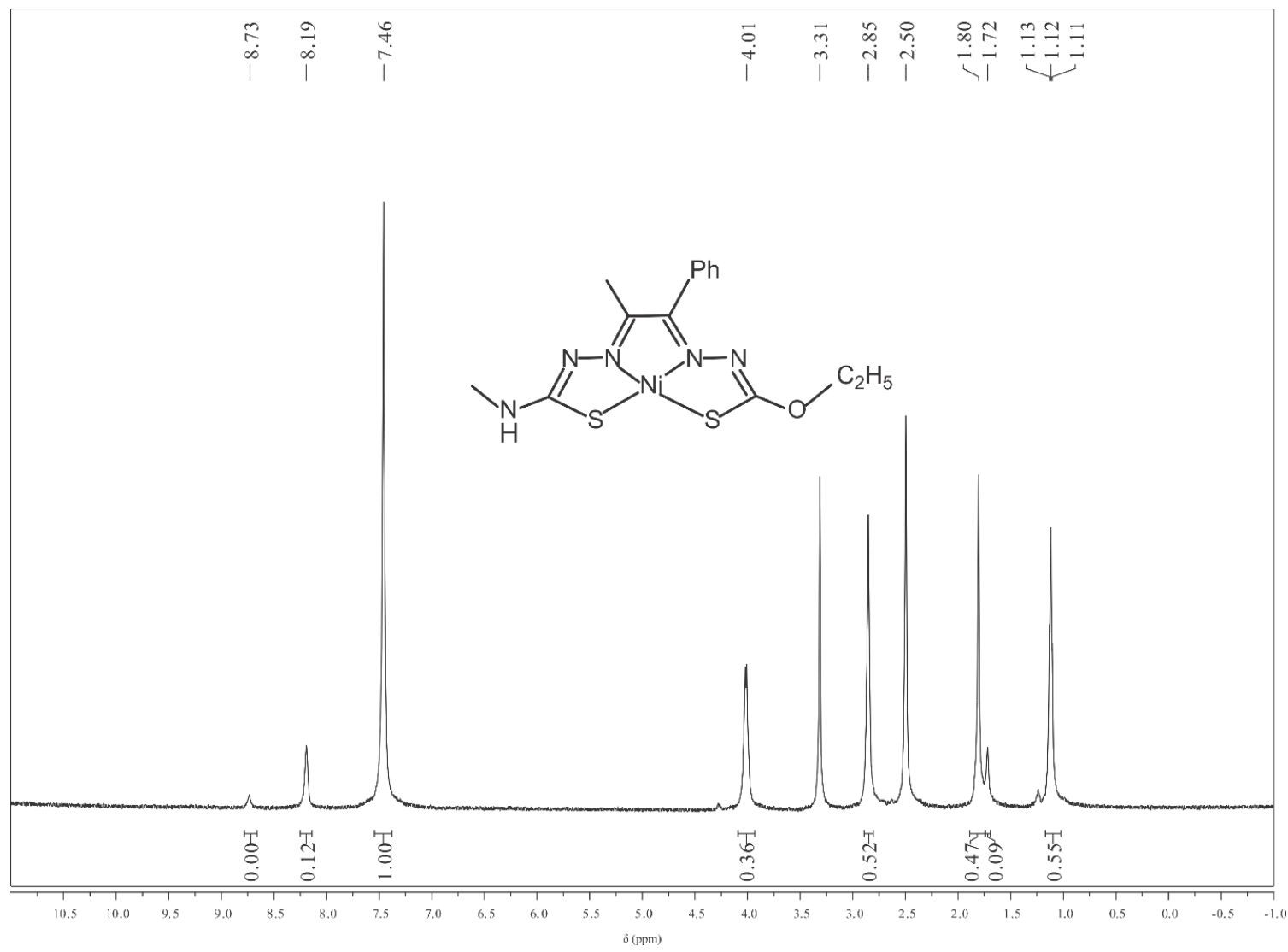

Figure A59. ${ }^{1} \mathrm{H}$ NMR $(400 \mathrm{MHz})$ of 24 in $\mathrm{DMSO}-\mathrm{d}_{6}$.

$\delta 1.12(\mathrm{t}, 3 \mathrm{H}) 1.80(\mathrm{~s}, 3 \mathrm{H}) 2.85(\mathrm{~s}, 3 \mathrm{H}) 7.46(\mathrm{br}, 5 \mathrm{H}) 8.19(\mathrm{~s}, 1 \mathrm{H})$

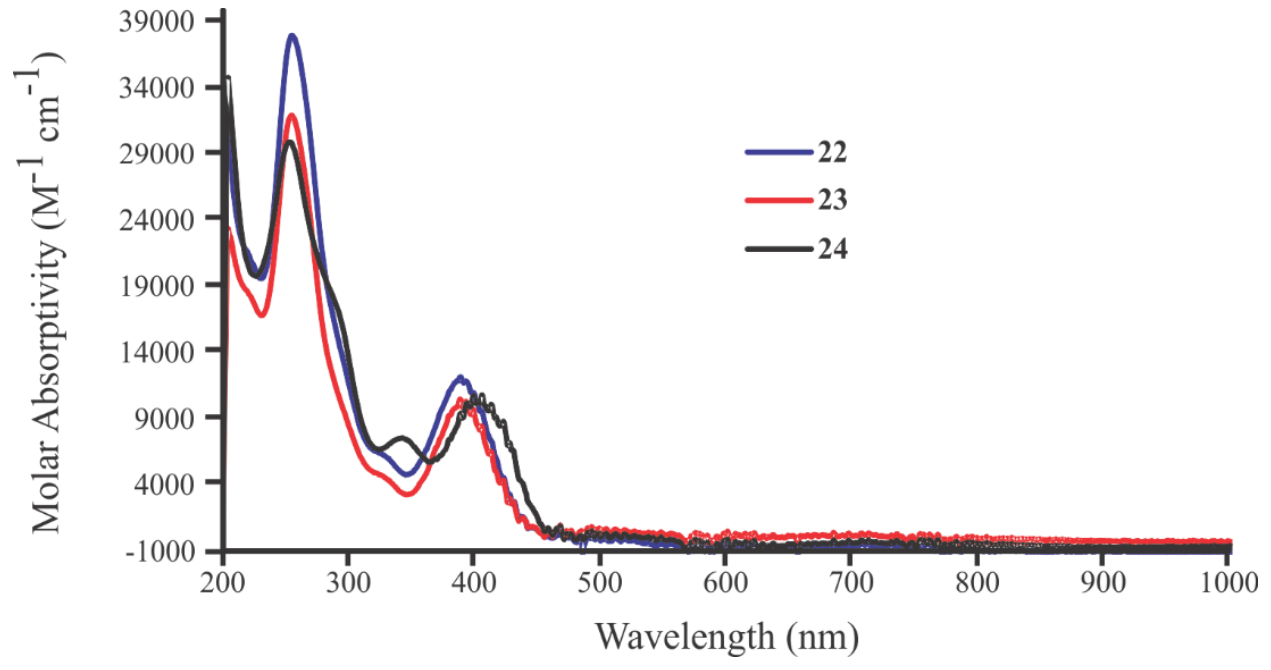

Figure A60. Electronic spectra of Ni complexes 22 (blue trace), 23 (red trace), and 24 (black trace) in acetonitrile 


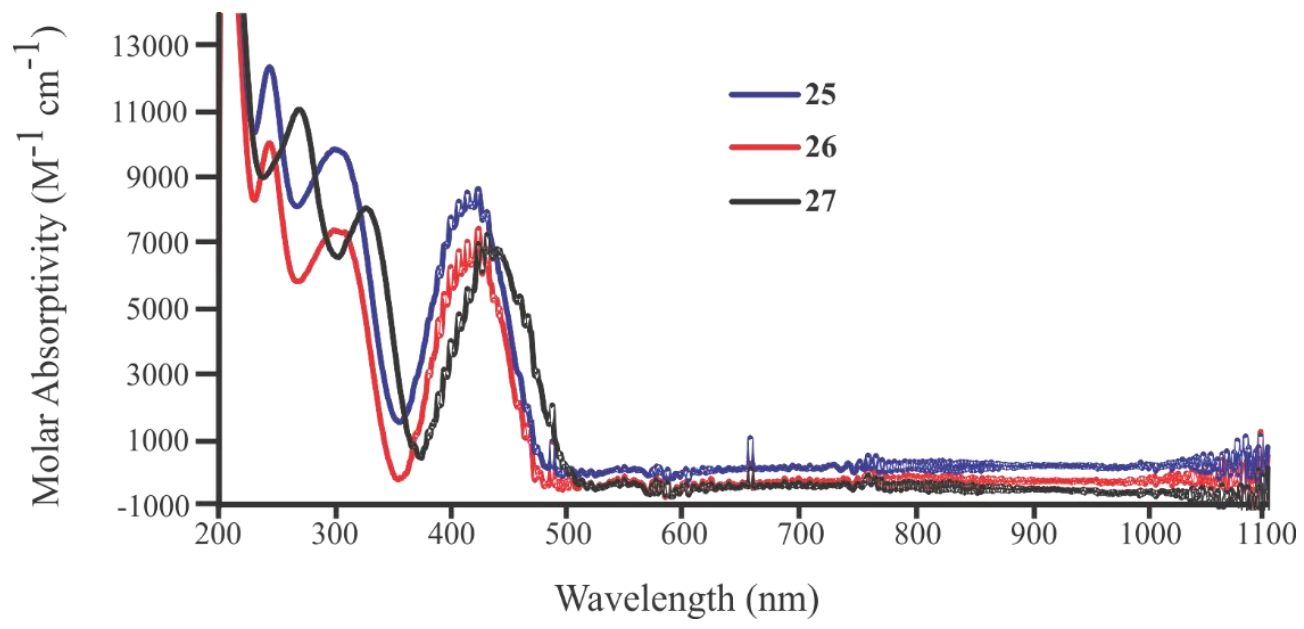

Figure A61. Electronic spectra of Zn complexes 25 (blue trace), 26 (red trace), and 27 (black trace) in acetonitrile 


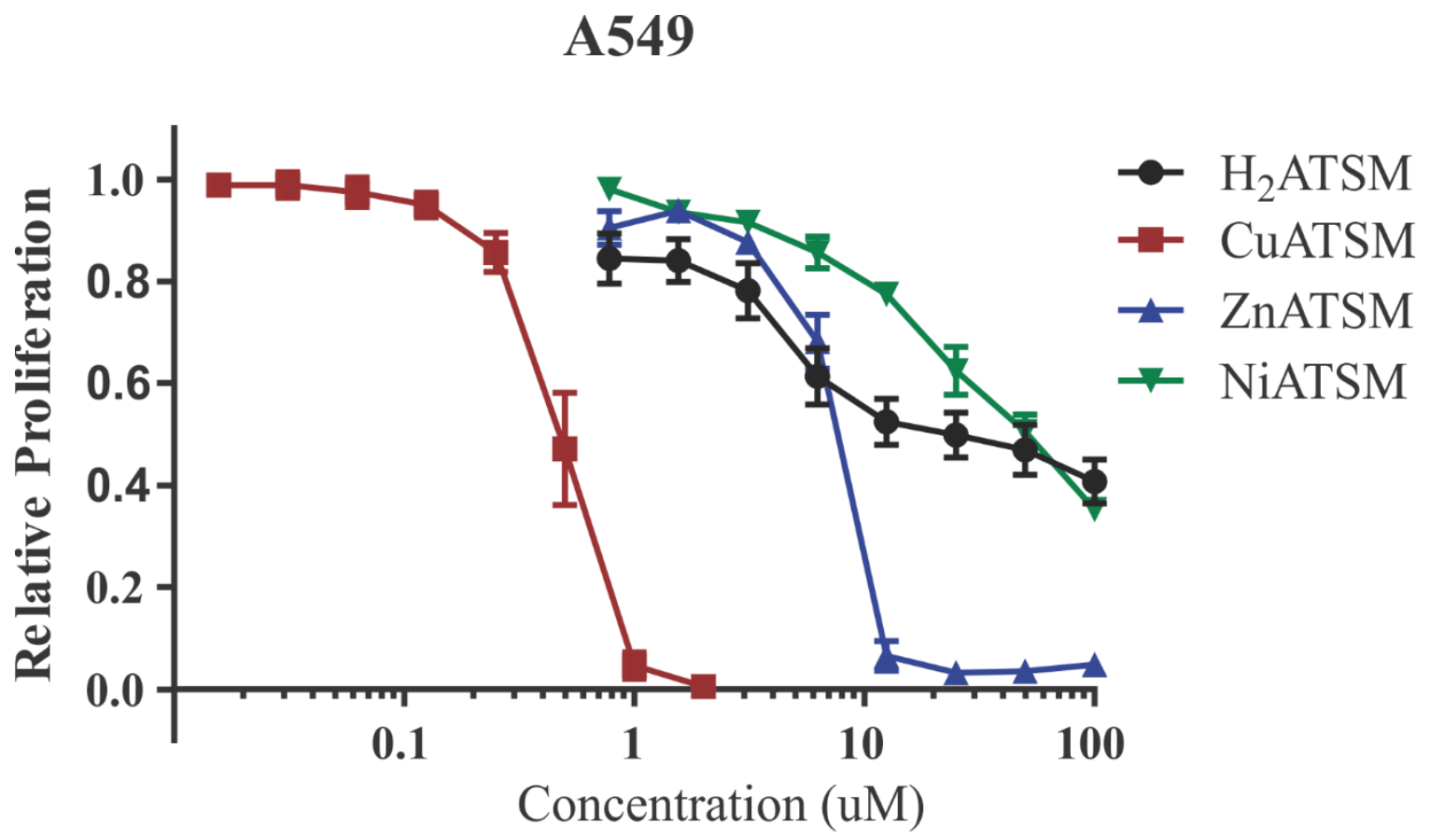

\section{IMR90}

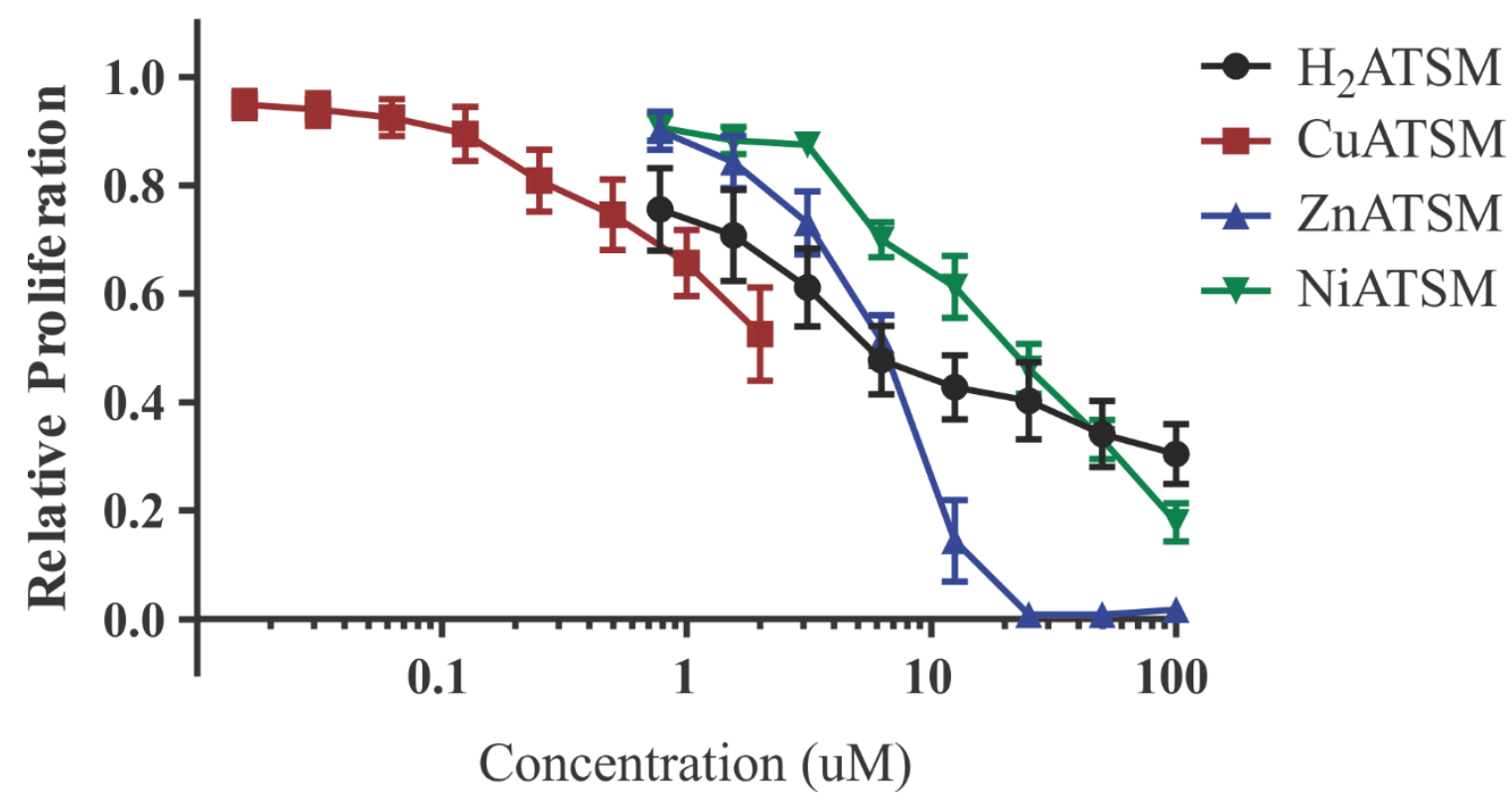

Figure A62. MTT assay results of cell lines A549 (top) and IMR90 (bottom) for the free ligand $\mathrm{H}_{2}$ ATSM (black trace), CuATSM (red trace), Zn complex (blue trace), and NiATSM (green trace). 


\title{
CURRICULUM VITAE
}

\section{Nicholas Vishnosky}

2402 Emil Ave

Louisville, KY- 40217, USA
001-860-559-9919 (cell)

001-502-852-6610 (lab)

Nicholas.vishnosky@louisville.edu

\section{Education}

University of Louisville, Louisville, KY, USA

$(\mathrm{GPA}=3.50 / 4.00)$

2013 - Present

Ph.D., Chemistry - Inorganic Chemistry

Dissertation Topic: Synthesis, characterization, and evaluation of metal complexes for selective targeting and anti-proliferative effect on cancer cells and their hydrogen evolution catalytic properties.

Advisor: Prof. Craig A. Grapperhaus

\section{Regenerative Medicine Research Center, Sichuan University, Chengdu 610041, Sichuan Province, P.R. China}

Summers 2015, 2016, 2017 Visiting Student

Collaborator: Prof. James Kang

Research Topic: Development of selective copper chelators for various in vivo applications such as imaging and delivery.

\section{Marist College, Poughkeepsie, NY, USA}

$(G P A=3.10 / 4.00$

\author{
$2009-2013$ \\ B.S. Chemistry
}

Research Topic: Effect of $\mathrm{pH}$ and mediators on xylanase activity

Advisor: Prof. Elisa Woolridge

\section{Research Skills}

Organic synthesis and inorganic synthesis under ambient and air-free conditions

Spectroscopic analysis and interpretation, including UV-visible spectroscopy, Infrared spectroscopy, electrochemistry, mass spectroscopy, electron paramagnetic resonance (EPR), multinuclear magnetic resonance (NMR), gas chromatography, fluorimetry, GC-MS, and HPLC.

Microsoft office program suite, Chem Draw, Endnote, Reaxsys, SciFinder, Corel Draw Laboratory equipment maintenance including glove box, vacuum pumps, solvent purification system, hazardous waste disposal, and Schlenk line

Familiar with crystallography (single crystal diffraction) and can solve small molecule structures using SHELX 


\section{Publications}

Vishnosky, N. S.; Mashuta, M. S.; Buchanan, R. M.; Grapperhaus, C. A., Syntheses, structures, and electrochemical studies of N,N'-bis(alkylthiocarbamate)butane-2,3-diimine $\mathrm{Cu}$ (II) complexes as pendent alkoxy derivatives of Cu(ATSM). Inorg. Chim. Acta 2017, 461, 45-51.

U.S. patent application PCT/US2018/030765 COMPOUNDS, COMPOSITIONS, METHODS FOR TREATING DISEASES,AND METHODS FOR PREPARING COMPOUNDS

Vishnosky, N. S.; Gupta, A.; Jain, R,; Mashuta, M. S.; Gupta, G.; Buchanan, R. M.; Grapperhaus, C. A., Effect of Stacking Interactions on the Translation of Structurally Related Bis-thiosemicarbazone Ni(II) HER Catalysts to Modified Electrode Surfaces.

Under revision for submission to Inorganic Chemistry

Vishnosky, N. S.; Andres, S; Mashuta, M. S.; Bates, P.; Buchanan, R. M.; Grapperhaus, C. A.,

Anticipated submission May 2019 to Inorganic Chemistry

Badon, M. M.; Tekverk, D. G.; Vishnosky, N. S.; Woolridge, E. M., Exploring the oxidative tolerance of xylanase.

Accepted and under revision

\section{Presentations}

Vishnosky, N. S.; Mashuta, M. S.; Buchanan, R. M.; Grapperhaus, C. A., "Syntheses, structures, and electrochemical studies of N,N'-bis(alkylthiocarbamate)butane-2,3-diimine Cu(II) complexes as pendent alkoxy derivatives of CU(ATSM)" ACS national meeting, San Francisco, CA (Spring 2017) (poster)

Vishnosky, N. S.; Mashuta, M. S.; Buchanan, R. M.; Grapperhaus, C. A., "Syntheses, structures, and electrochemical studies of $N, N^{\prime}$-bis(alkylthiocarbamate)butane-2,3-diimine Cu(II) complexes as pendent alkoxy derivatives of Cu(ATSM)" Kentucky Academy of Science, Louisville, KY (Fall 2016) (poster)

Vishnosky, N. S.; Gupta, A. J.; Jain, R.; Mashuta, M. S.; Gupta, G.; Buchanan, R. M.; Grapperhaus, C. A.Heterogeneous Hydrogen Evolution with Novel Nickel ATSM Catalysts and the Effect of Surface Morphology" ACS national meeting, Boston, MA (Fall 2018) (poster)

Sichuan University, Chengdu, Sichuan Province, P.R. China (Summer 2016) (literature seminar)

\section{Collaborators}

Dr. Paula Bates (Associate Professor of Medicine)

Dr. James Kang (Department of Pharmacology and Toxicology)

Dr. Gautam Gupta (Associate Professor, Chemical Engineering)

\section{Teaching Experience}

University of Louisville, Louisville, KY, USA

2013-2018 Graduate Teaching Assistant General Chemistry I\&II (Chem-201\&202)

Marist College, Poughkeepsie, NY, USA

$2011-2013 \quad$ Chemistry Tutor General and Organic Chemistry 


\title{
Professional Memberships
}

\author{
2011 - Present American Chemical Society
}

\section{Lanquaqes}

Spanish (Limited working proficiency, ILR level 2)

Chinese (Elementary proficiency, ILR Level 1)

\section{Underaraduate Student Mentorship}

1. Joel Reihmer (NSF-REU student) Summer 2014

2. Alan Weihe Spring \& Summer 2016

3. Maryssa Becker, Spring 2018

\section{Previous Emplovment}

Dunkin Donuts/Baskin Robbins, Glastonbury, CT

$05 / 2011-08 / 2011$

Crew Member

- Greeted customers in a friendly manner

- Prepared and served beverages, ice cream specialties and various food items

- Delivered fast and prompt service

- Accurately handled cash for purchased food items

- Maintained clean environment by sweeping, mopping and removing garbage

Muscular Dystrophy Association, Glastonbury, CT

$06 / 2010-08 / 2010$

Volunteer for Local Agency

- Assisted in charity fundraising event by taking inventory of sponsoring signs, selling raffle tickets, and distributing tokens of appreciation

- Organized mass mailings regarding upcoming events and fundraisers, and notification of outstanding donations

Ted's Supermarket, Hebron, CT

$06 / 2008-08 / 2008$

Grocery Bagger

- Accommodated customers by bagging groceries and loading into their cars

- Organized stocked shelves

- Closing duties included putting meats in freezer

\section{Other Activities}

Marist College:Treasurer of Resident Housing Council, Tutor, Intramural Soccer, Booster Club, Habitat for Humanity, Marist International Students Club, ACS Club

East Catholic High School: Jazz Band, Soccer Team, Indoor \& Outdoor Track Teams, Cross Country Team

\section{Marlborough Soccer Club}

